\title{
Personalised care planning for adults with chronic or long- term health conditions (Review)
}

\author{
Coulter A, Entwistle VA, Eccles A, Ryan S, Shepperd S, Perera R
}

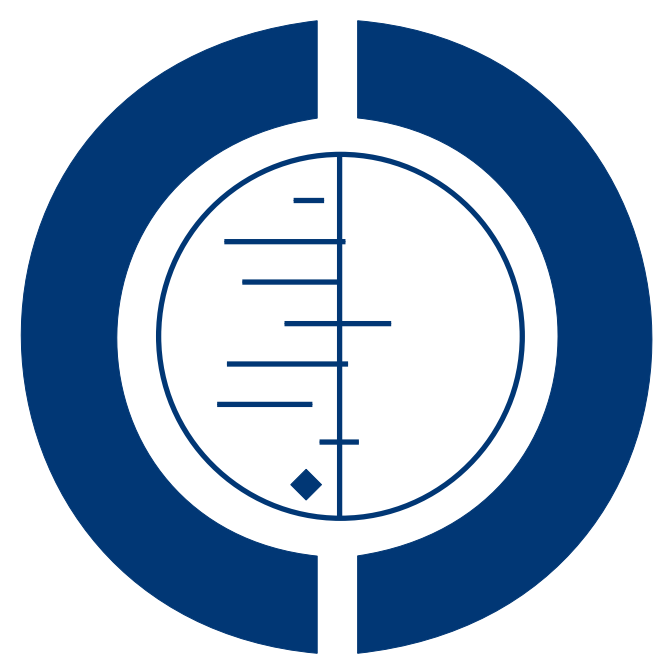

\section{THE COCHRANE COLLABORATION $^{\circledR}$}

This is a reprint of a Cochrane review, prepared and maintained by The Cochrane Collaboration and published in The Cochrane Library 2015, Issue 3

http://www.thecochranelibrary.com

\section{WILEY}

Personalised care planning for adults with chronic or long-term health conditions (Review)

Copyright $\odot 2015$ The Cochrane Collaboration. Published by John Wiley \& Sons, Ltd. 
TABLE OF CONTENTS

HEADER

ABSTRACT

PLAIN LANGUAGE SUMMARY . . . . . . . . . . . . . . . . . . . . . . . . . . . . . . . . . . .

SUMMARY OF FINDINGS FOR THE MAIN COMPARISON . . . . . . . . . . . . . . . . . . . . . . . 4

BACKGROUND . . . . . . . . . . . . . . . . . . . . . . . . . . . . . . . . . . . . . 6

Figure 1. . . . . . . . . . . . . . . . . . . . . . . . . . . . . . . . . . . . . . . . . 47

OBJECTIVES . . . . . . . . . . . . . . . . . . . . . . . . . . . . . . . . . . . . . 10

METHODS . . . . . . . . . . . . . . . . . . . . . . . . . . . . . . . . . . . . . . 10

RESULTS . . . . . . . . . . . . . . . . . . . . . . . . . . . . . . . . . . . . . . . 15

Figure 2. . . . . . . . . . . . . . . . . . . . . . . . . . . . . . . . . . . . . . 16

Figure 3. . . . . . . . . . . . . . . . . . . . . . . . . . . . . . . . . . . . . . 19

Figure $4 . \quad$. . . . . . . . . . . . . . . . . . . . . . . . . . . . . . . . . . . . . 20

DISCUSSION . . . . . . . . . . . . . . . . . . . . . . . . . . . . . . . . . . . . . . . . . . . . . .

AUTHORS' CONCLUSIONS . . . . . . . . . . . . . . . . . . . . . . . . . . . . . . . . . . . . . .

ACKNOWLEDGEMENTS . . . . . . . . . . . . . . . . . . . . . . . . . . . . . . . . . . . . . . .

REFERENCES . . . . . . . . . . . . . . . . . . . . . . . . . . . . . . . . . . . . . . 29

CHARACTERISTICS OF STUDIES . . . . . . . . . . . . . . . . . . . . . . . . . . . . . . 39

DATA AND ANALYSES . . . . . . . . . . . . . . . . . . . . . . . . . . . . . . . . . . . . . . . . . . . . . . . . . . . .

Analysis 1.1. Comparison 1 Physical health (personalised care planning vs usual care), Outcome $1 \mathrm{HbA1c}$ (change). . 85

Analysis 1.2. Comparison 1 Physical health (personalised care planning vs usual care), Outcome 2 SBP (change). . .86

Analysis 1.3. Comparison 1 Physical health (personalised care planning vs usual care), Outcome 3 DBP (change). $\quad .87$

Analysis 1.4. Comparison 1 Physical health (personalised care planning vs usual care), Outcome 4 Cholesterol (change). 88

Analysis 1.5. Comparison 1 Physical health (personalised care planning vs usual care), Outcome 5 BMI (change). .89

Analysis 2.1. Comparison 2 Psychological health (personalised care planning vs usual care), Outcome 1 Depression. . 90

Analysis 3.1. Comparison 3 Subjective health status (personalised care planning vs usual care), Outcome 1 Generic health status (physical).

Analysis 3.2. Comparison 3 Subjective health status (personalised care planning vs usual care), Outcome 2 Generic health status (mental).

Analysis 3.3. Comparison 3 Subjective health status (personalised care planning vs usual care), Outcome 3 Conditionspecific health status.

Analysis 4.1. Comparison 4 Self-management capabilities (personalised care planning vs usual care), Outcome 1 Self efficacy.

Analysis 5.1. Comparison 5 Health-related behaviours (personalised care planning vs usual care), Outcome 1 Exercise.

Analysis 6.1. Comparison 6 Self-care activities (personalised care planning vs usual care), Outcome 1 Self care (days per week).

Analysis 7.1. Comparison 7 Type of intervention (HbA1c) (extended vs limited), Outcome 1 HbAlc (change).

Analysis 8.1. Comparison 8 Type of intervention (HbA1c) (high intensity vs low intensity), Outcome 1 HbA1c (change).

Analysis 9.1. Comparison 9 Type of intervention (HbA1c) (integrated vs not integrated), Outcome $1 \mathrm{HbA1c}$ (change).

ADDITIONAL TABLES .

APPENDICES

WHAT'S NEW . . . . . . . . . . . . . . . . . . . . . . . . . . . . . . . . . . . . .

CONTRIBUTIONS OF AUTHORS . . . . . . . . . . . . . . . . . . . . . . . . . . . . . . . . . . .

DECLARATIONS OF INTEREST . . . . . . . . . . . . . . . . . . . . . . . . . . . . . . . . . . . . . .

SOURCES OF SUPPORT . . . . . . . . . . . . . . . . . . . . . . . . . . . . . . . . . . . . . . . . . . . . . . . . . . . . . .

DIFFERENCES BETWEEN PROTOCOL AND REVIEW . . . . . . . . . . . . . . . . . . . . . . . . . 129

Personalised care planning for adults with chronic or long-term health conditions (Review)

Copyright () 2015 The Cochrane Collaboration. Published by John Wiley \& Sons, Ltd. 


\title{
[Intervention Review] \\ Personalised care planning for adults with chronic or long- term health conditions
}

\author{
Angela Coulter ${ }^{1}$, Vikki A Entwistle ${ }^{2}$, Abi Eccles $^{3}$, Sara Ryan ${ }^{4}$, Sasha Shepperd ${ }^{5}$, Rafael Perera ${ }^{6}$ \\ ${ }^{1}$ Health Services Research Unit, Nuffield Department of Population Health, University of Oxford, Oxford, UK. ${ }^{2}$ Health Services \\ Research Unit, University of Aberdeen, Aberdeen, UK. ${ }^{3}$ Department of Primary Care Health Sciences, University of Oxford, Oxford, \\ UK. ${ }^{4}$ Quality and Outcomes Research Unit and Health Experiences Research Group, University of Oxford, Oxford, UK. ${ }^{5}$ Nuffield \\ Department of Population Health, University of Oxford, Oxford, UK. ${ }^{6}$ Nuffield Department of Primary Care Health Sciences, \\ University of Oxford, Oxford, UK \\ Contact address: Angela Coulter, Health Services Research Unit, Nuffield Department of Population Health, University of Oxford, \\ Old Road Campus, Headington, Oxford, OX3 7LF, UK. angela.coulter@dph.ox.ac.uk.
}

Editorial group: Cochrane Consumers and Communication Group.

Publication status and date: Edited (no change to conclusions), published in Issue 3, 2015.

Review content assessed as up-to-date: 1 January 2014.

Citation: Coulter A, Entwistle VA, Eccles A, Ryan S, Shepperd S, Perera R. Personalised care planning for adults with chronic or long-term health conditions. Cochrane Database of Systematic Reviews 2015, Issue 3. Art. No.: CD010523. DOI: 10.1002/14651858.CD010523.pub2.

Copyright (C) 2015 The Cochrane Collaboration. Published by John Wiley \& Sons, Ltd.

\begin{abstract}
A B S T R A C T
Background

Personalised care planning is a collaborative process used in chronic condition management in which patients and clinicians identify and discuss problems caused by or related to the patient's condition, and develop a plan for tackling these. In essence it is a conversation, or series of conversations, in which they jointly agree goals and actions for managing the patient's condition.
\end{abstract}

\section{Objectives}

To assess the effects of personalised care planning for adults with long-term health conditions compared to usual care (i.e. forms of care in which active involvement of patients in treatment and management decisions is not explicitly attempted or achieved).

\section{Search methods}

We searched the Cochrane Central Register of Controlled Trials (CENTRAL), MEDLINE, EMBASE, PsycINFO, ProQuest, clinicaltrials.gov and WHO International Clinical Trials Registry Platform to July 2013.

\section{Selection criteria}

We included randomised controlled trials and cluster-randomised trials involving adults with long-term conditions where the intervention included collaborative (between individual patients and clinicians) goal setting and action planning. We excluded studies where there was little or no opportunity for the patient to have meaningful influence on goal selection, choice of treatment or support package, or both.

\section{Data collection and analysis}

Two of three review authors independently screened citations for inclusion, extracted data, and assessed risk of bias. The primary outcomes were effects on physical health, psychological health, subjective health status, and capabilities for self management. Secondary outcomes included effects on health-related behaviours, resource use and costs, and type of intervention. A patient advisory group of people with experience of living with long-term conditions advised on various aspects of the review, including the protocol, selection of outcome measures and emerging findings.

Personalised care planning for adults with chronic or long-term health conditions (Review)

Copyright $\odot 2015$ The Cochrane Collaboration. Published by John Wiley \& Sons, Ltd. 


\section{Main results}

We included 19 studies involving a total of 10,856 participants. Twelve of these studies focused on diabetes, three on mental health, one on heart failure, one on end-stage renal disease, one on asthma, and one on various chronic conditions. All 19 studies included components that were intended to support behaviour change among patients, involving either face-to-face or telephone support. All but three of the personalised care planning interventions took place in primary care or community settings; the remaining three were located in hospital clinics. There was some concern about risk of bias for each of the included studies in respect of one or more criteria, usually due to inadequate or unclear descriptions of research methods.

\section{Physical health}

Nine studies measured glycated haemoglobin (HbA1c), giving a combined mean difference (MD) between intervention and control of $-0.24 \%$ (95\% confidence interval (CI) -0.35 to -0.14 ), a small positive effect in favour of personalised care planning compared to usual care (moderate quality evidence).

Six studies measured systolic blood pressure, a combined mean difference of $-2.64 \mathrm{~mm} / \mathrm{Hg}$ ( $95 \%$ CI -4.47 to -0.82 ) favouring personalised care (moderate quality evidence). The pooled results from four studies showed no significant effect on diastolic blood pressure, MD $-0.71 \mathrm{~mm} / \mathrm{Hg}(95 \% \mathrm{CI}-2.26$ to 0.84$)$.

We found no evidence of an effect on cholesterol (LDL-C), standardised mean difference (SMD) 0.01 (95\% CI -0.09 to 0.11 ) (five studies) or body mass index, MD -0.11 (95\% CI -0.35 to 0.13 ) (four studies).

A single study of people with asthma reported that personalised care planning led to improvements in lung function and asthma control.

\section{Psychological health}

Six studies measured depression. We were able to pool results from five of these, giving an SMD of -0.36 (95\% CI -0.52 to -0.20 ), a small effect in favour of personalised care (moderate quality evidence). The remaining study found greater improvement in the control group than the intervention group.

Four other studies used a variety of psychological measures that were conceptually different so could not be pooled. Of these, three found greater improvement for the personalised care group than the usual care group and one was too small to detect differences in outcomes.

\section{Subjective health status}

Ten studies used various patient-reported measures of health status (or health-related quality of life), including both generic health status measures and condition-specific ones. We were able to pool data from three studies that used the SF-36 or SF-12, but found no effect on the physical component summary score SMD 0.16 (95\% CI -0.05 to 0.38 ) or the mental component summary score SMD 0.07 ( $95 \%$ CI -0.15 to 0.28 ) (moderate quality evidence). Of the three other studies that measured generic health status, two found improvements related to personalised care and one did not.

Four studies measured condition-specific health status. The combined results showed no difference between the intervention and control groups, SMD -0.01 (95\% CI -0.11 to 0.10$)$ (moderate quality evidence).

\section{Self-management capabilities}

Nine studies looked at the effect of personalised care on self-management capabilities using a variety of outcome measures, but they focused primarily on self efficacy. We were able to pool results from five studies that measured self efficacy, giving a small positive result in favour of personalised care planning: SMD 0.25 (95\% CI 0.07 to 0.43 ) (moderate quality evidence).

A further five studies measured other attributes that contribute to self-management capabilities. The results from these were mixed: two studies found evidence of an effect on patient activation, one found an effect on empowerment, and one found improvements in perceived interpersonal support.

\section{Other outcomes}

Pooled data from five studies on exercise levels showed no effect due to personalised care planning, but there was a positive effect on people's self-reported ability to carry out self-care activities: SMD 0.35 (95\% CI 0.17 to 0.52 ).

We found no evidence of adverse effects due to personalised care planning.

Personalised care planning for adults with chronic or long-term health conditions (Review) 
The effects of personalised care planning were greater when more stages of the care planning cycle were completed, when contacts between patients and health professionals were more frequent, and when the patient's usual clinician was involved in the process.

\section{Authors' conclusions}

Personalised care planning leads to improvements in certain indicators of physical and psychological health status, and people's capability to self-manage their condition when compared to usual care. The effects are not large, but they appear greater when the intervention is more comprehensive, more intensive, and better integrated into routine care.

\section{PLAIN LANGUAGE SUMMARY}

\section{Effects of personalised care planning for people with long-term conditions}

\section{Background}

People with long-term health conditions play an important part in managing their own health. But some of the tasks involved can be complicated, and require confidence and skill. Such tasks include taking medicines properly, monitoring symptoms, adopting or maintaining healthy lifestyles, managing their emotions, solving practical problems, knowing when and how to seek medical advice or community support, and coping with the impact of the condition(s) on their daily lives. Personalised care planning aims to provide support from health professionals that is tailored to the needs of individual patients. Such support recognises patients' concerns, and helps them become more able to manage their own health. Personalised care planning is a conversation, or series of conversations, between a patient and a clinician when they jointly agree on goals and actions for managing the patient's health problems.

\section{Review question}

We carried out this systematic review to find out whether a personalised approach, in which patients are encouraged to participate in setting goals and action plans and determining their support needs, leads to better outcomes than when these decisions are taken by health professionals alone.

\section{Results}

We found 19 randomised trials published before July 2013 that addressed this issue, involving 10,856 participants with conditions such as diabetes, mental health problems, heart failure, kidney disease, and asthma. The studies looked at a range of different interventions designed to involve patients and support self management. We combined and summarised results from studies that measured similar outcomes and found that involvement in personalised care planning probably led to small improvements in some indicators of physical health (better blood glucose levels, lower blood pressure measurements among people with diabetes, and control of asthma). It also probably reduced symptoms of depression, and improved people's confidence and skills to manage their health. We observed no effect on cholesterol, body mass index or quality of life. We found no evidence of any harms arising from personalised care planning. We found that the process worked best when it included preparation, record-sharing, care co-ordination and review, involved more intensive support from health professionals, and was integrated into routine care. However, the quality of evidence was only moderate, meaning that further research might change these findings.

\section{Conclusion}

We concluded that personalised care planning is a promising approach that offers the potential to provide effective help to patients, leading to better health outcomes. More research is needed to work out which aspects are most effective for specific patient groups. 
SUMMARY OF FINDINGS FOR THE MAIN COMPARISON [Explanation]

Personalised care planning compared with usual care

Patient or population: Adult patients with long-term health conditions

Settings: All settings

Intervention: Personalised care planning

Comparison: Usual care or enhanced usual care

\begin{tabular}{|c|c|c|c|c|}
\hline \multirow[t]{2}{*}{ Outcomes } & $\begin{array}{l}\text { Illustrative comparative } \\
\text { effect sizes* }(95 \% \text { CI) }\end{array}$ & \multirow[t]{2}{*}{$\begin{array}{l}\text { No of participants } \\
\text { (studies) }\end{array}$} & \multirow[t]{2}{*}{$\begin{array}{l}\text { Quality of the evidence } \\
\text { (GRADE) }\end{array}$} & \multirow[t]{2}{*}{ Comments } \\
\hline & $\begin{array}{l}\text { Usual care (control) vs } \\
\text { personalised care plan- } \\
\text { ning (intervention) }\end{array}$ & & & \\
\hline 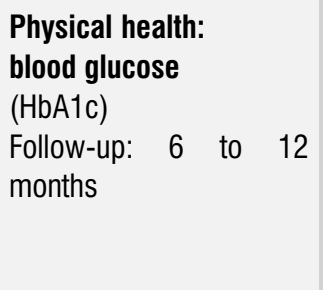 & $\begin{array}{l}\text { The mean difference in } \\
\text { blood glucose was } \mathbf{0 .} \\
\mathbf{2 4 \%} \text { lower (better) in } \\
\text { the intervention groups } \\
\text { than in the control groups } \\
\text { (95\% Cl } 0.35 \text { to } 0.14 \\
\text { lower) }\end{array}$ & $\begin{array}{l}1916 \\
\text { (9 studies) }\end{array}$ & $\begin{array}{l}\oplus \oplus \oplus \bigcirc \\
\text { moderate } \\
\text { (variation in intervention } \\
\text { types led to significant } \\
\text { heterogeneity and risk of } \\
\text { bias was unclear) }\end{array}$ & \\
\hline $\begin{array}{l}\text { Physical health: } \\
\text { systolic blood pressure } \\
\text { Follow-up: } 6 \text { to } 12 \\
\text { months }\end{array}$ & $\begin{array}{l}\text { The mean difference in } \\
\text { systolic blood pressure } \\
\text { was } 2.64 \mathrm{~mm} / \mathrm{Hg} \text { lower } \\
\text { (better) in the interven- } \\
\text { tion groups than in the } \\
\text { control groups ( } 95 \% \mathrm{Cl} 4 \text {. } \\
47 \text { to } 0.82 \text { lower) }\end{array}$ & $\begin{array}{l}1200 \\
\text { (6 studies) }\end{array}$ & $\begin{array}{l}\oplus \oplus \oplus \bigcirc \\
\text { moderate } \\
\text { (variation in intervention } \\
\text { types led to significant } \\
\text { heterogeneity and risk of } \\
\text { bias was unclear) }\end{array}$ & \\
\hline $\begin{array}{l}\text { Physical health: } \\
\text { cholesterol } \\
\text { (LDL-C) } \\
\begin{array}{llll} & & \\
\text { Follow-up: } 6 & \text { to } & 12 \\
\text { months } & & & \end{array}\end{array}$ & $\begin{array}{l}\text { The standardised mean } \\
\text { difference in LDL choles- } \\
\text { terol did not differ be- } \\
\text { tween the intervention } \\
\text { and control groups: } 0 \text {. } \\
01 \text { standard deviations } \\
\text { ( } 95 \% \mathrm{Cl}-0.09 \text { to } 0.11 \text { ) }\end{array}$ & $\begin{array}{l}1545 \\
\text { (5 studies) }\end{array}$ & $\begin{array}{l}\oplus \oplus \oplus \bigcirc \\
\text { moderate } \\
\text { (results were inconsis- } \\
\text { tent) }\end{array}$ & \\
\hline $\begin{array}{l}\text { Psychological health: } \\
\text { depression } \\
\text { (PHQ- } \\
\text { 9, SCL-20, Beck Depres- } \\
\text { sion Inventory, CES-D) } \\
\text { Follow-up: } 1.5 \text { to } 12 \\
\text { months }\end{array}$ & $\begin{array}{l}\text { The standardised mean } \\
\text { difference in depression } \\
\text { scores was } \mathbf{0 . 3 6} \text { stan- } \\
\text { dard deviations lower } \\
\text { (better) in the interven- } \\
\text { tion groups than in the } \\
\text { control groups ( } 95 \% \mathrm{Cl} 0 \text {. } \\
52 \text { to } 0.20 \text { lower), a small } \\
\text { effect in favour of person- } \\
\text { alised care planning }\end{array}$ & $\begin{array}{l}599 \\
\text { (5 studies) }\end{array}$ & $\begin{array}{l}\oplus \oplus \oplus \bigcirc \\
\text { moderate } \\
\text { (multiple outcome mea- } \\
\text { sures) }\end{array}$ & $\begin{array}{l}\text { In addition, } 3 \text { out of } 4 \\
\text { studies that used con- } \\
\text { ceptually different mea- } \\
\text { sures of psychological } \\
\text { outcomes (and so could } \\
\text { not be pooled) reported } \\
\text { better outcomes for the } \\
\text { intervention groups than } \\
\text { the control groups. The } \\
\text { remaining study was too }\end{array}$ \\
\hline
\end{tabular}

Personalised care planning for adults with chronic or long-term health conditions (Review)

Copyright @ 2015 The Cochrane Collaboration. Published by John Wiley \& Sons, Ltd. 
small to detect an effect

\begin{tabular}{|c|c|c|c|}
\hline & & & small to detect an effect \\
\hline $\begin{array}{l}\text { Subjective health status: } \\
\text { condition-specific } \\
\text { (PAID-2, Illness Intrusive- } \\
\text { ness, AQLQ) } \\
\text { Follow-up: } 12 \text { months }\end{array}$ & $\begin{array}{l}\text { The standardised mean } 1330 \\
\text { difference in condition- (4 studies) } \\
\text { specific health status } \\
\text { scores did not differ be- } \\
\text { tween the intervention } \\
\text { and control groups: }-0 . \\
\text { 01 standard deviations } \\
\text { (95\% Cl }-0.11 \text { to } 0.10 \text { ) }\end{array}$ & $\begin{array}{l}\oplus \oplus \oplus \bigcirc \\
\text { moderate } \\
\text { (variation in intervention } \\
\text { types led to significant } \\
\text { heterogeneity) }\end{array}$ & $\begin{array}{l}\text { Three studies that mea- } \\
\text { sured generic health sta- } \\
\text { tus (SF-36 or SF-12) } \\
\text { found no difference be- } \\
\text { tween intervention and } \\
\text { control groups: physical } \\
\text { component score SMD } 0 . \\
16 \text { ( } 95 \% \mathrm{Cl}-0.05 \text { to } 0 . \\
38 \text { ); mental component } \\
\text { score SMD } 0.07 \text { (95\% Cl } \\
-0.15 \text { to } 0.28 \text { ) }\end{array}$ \\
\hline $\begin{array}{l}\text { Self-management capa- } \\
\text { bilities: self efficacy } \\
\text { (Stanford, } \\
\text { PCDS) } \\
\begin{array}{l}\text { Follow-up: } 1.5 \text { to } 12 \\
\text { months }\end{array}\end{array}$ & $\begin{array}{l}\text { The standardised mean } 471 \\
\text { difference in self-efficacy ( } 5 \text { studies) } \\
\text { scores was } \mathbf{0 . 2 5} \text { stan- } \\
\text { dard deviations higher } \\
\text { (better) in the interven- } \\
\text { tion groups than in the } \\
\text { control groups ( } 95 \% \mathrm{Cl} 0 \text {. } \\
07 \text { to } 0.43 \text { higher), a small } \\
\text { effect in favour of person- } \\
\text { alised care planning }\end{array}$ & $\begin{array}{l}\oplus \oplus \oplus \bigcirc \\
\text { moderate } \\
\text { (variation in intervention } \\
\text { types led to significant } \\
\text { heterogeneity and risk of } \\
\text { bias was unclear) }\end{array}$ & $\begin{array}{l}\text { Mixed effects were found } \\
\text { in } 5 \text { studies that mea- } \\
\text { sured other attributes that } \\
\text { contribute to self-man- } \\
\text { agement capabilities. We } \\
\text { also found a positive ef- } \\
\text { fect on performance of } \\
\text { self-care activities asso- } \\
\text { ciated with personalised } \\
\text { care planning, SMD 0.35 } \\
(95 \% \mathrm{Cl} 0.17 \text { to 0.52) }\end{array}$ \\
\hline $\begin{array}{l}\text { Harms associated with } \\
\text { personalised care plan- } \\
\text { ning }\end{array}$ & & & $\begin{array}{l}\text { Only } 1 \text { study reported any } \\
\text { adverse events (hospital- } \\
\text { isation and deaths), but } \\
\text { there were no differences } \\
\text { between intervention and } \\
\text { usual-care groups and no } \\
\text { reason to assume that } \\
\text { these were due to the in- } \\
\text { tervention }\end{array}$ \\
\hline
\end{tabular}

* Cl: Confidence interval

GRADE Working Group grades of evidence

High quality: Further research is very unlikely to change our confidence in the estimate of effect.

Moderate quality: Further research is likely to have an important impact on our confidence in the estimate of effect and may change the estimate.

Low quality: Further research is very likely to have an important impact on our confidence in the estimate of effect and is likely to change the estimate.

Very low quality: We are very uncertain about the estimate. 


\section{B A C K G R O U N D}

\section{Description of the condition}

The treatment and management of long-term health conditions (including those associated with physical, psychological, sensory, or cognitive problems) is the greatest challenge facing health systems around the world today (UN Secretary General 2011). Strategies used by health professionals to engage, support and empower people with long-term conditions have an important role in improving health outcomes (George Institute 2011; Wanless 2002). Patients who are better informed, more involved in decisions about their care and more 'activated' (i.e. recognise that they have an important role in self-managing their condition(s) and have the skills and confidence to do so) (Hibbard 2004) experience improved health and better quality of life (Michie 2003; Schmittdiel 2008). Strengthening patients' autonomy and capacity to selfmanage their health is pivotal to policymakers' attempts to achieve value for money, particularly in times of economic recession. Some policymakers hope that this may also help to tackle unacceptable health inequalities between socioeconomic groups (Department of Health 2009).

The Chronic Care Model, which has been highly influential internationally, stresses the need to transform health care for people with long-term health conditions from a system that is largely reactive, responding mainly when a person is sick, to one that is much more proactive, focused on supporting people's ability to self-manage their health (Epping-Jordan 2004; Nolte 2008; Wagner 1998). The model advocates an active role for patients, who are encouraged to become both more knowledgeable about factors affecting their condition(s) (including strategies for preventing exacerbations or ameliorating symptoms), and more actively involved in decisions about their care. The clinician's responsibility is to gauge the extent of the patient's knowledge, skills and confidence to selfmanage his or her health, to strengthen this where necessary, and to ensure that relevant interventions and support services are available (Department of Health 2011; Von Korff 1997; Year of Care 2011). At the heart of the model is an informed, active patient, supported by a well-prepared, proactive primary care team, working together to develop and implement a personalised care plan. The rising prevalence of multi-morbidity makes the search for effective ways of developing personalised approaches even more important. Demographic change and longer life expectancy mean that increasing numbers of people have more than one chronic condition, requiring specially tailored approaches to the management of complex combinations of conditions and treatment strategies (Barnett 2012). The specialty-led, single disease framework that characterises the organisation of most medical care is outdated. Ideally, care for people with multiple long-term conditions should be holistic: person-focused rather than disease-focused, and responsive to individuals' experiences of illness and treatment effects and their personal priorities (Mangin 2012).

In managing long-term health conditions, the aims are: to minimise the negative impacts and maximise the potential for improved functioning and well-being; to strengthen people's capabilities for self-managing their condition; to reduce health risks by improving health-related behaviours; and to minimise dependence on resource-intensive, costly health services. Personalised care planning is seen as a promising way to achieve these goals.

\section{Description of the intervention}

Personalised care planning aims to ensure that individuals' values and concerns shape the way long-term conditions are managed. Instead of focusing on a standard set of disease management processes determined by health professionals, this approach encourages patients to select treatment goals and to work with clinicians to determine their specific needs for treatment and support (Reuben 2012). The process involves a shift from reactive care (waiting for people to consult with symptoms) to a proactive approach in which patients are invited to attend specially scheduled care planning consultations. For the purpose of this review, we define personalised care planning as: an anticipatory (forwardlooking), negotiated discussion or series of discussions between a patient and a health professional (perhaps with other professional or family members present) to clarify goals, options and preferences and develop an agreed plan of action based on this mutual understanding.

In personalised care planning, patients and clinicians identify and discuss problems caused by or related to the patient's condition(s), giving due consideration to both clinical tests and treatments and the practical, social, and emotional effects of their condition(s) and treatment(s) on their daily lives. They then engage in a shared decision-making process involving goal setting and action planning, focused on determining priorities, agreeing realistic objectives, solving specific problems, and identifying relevant sources of support. In some cases a family member, carer/caregiver or friend may also be included in the discussion. Management options and support needs under discussion might include any or all of the following:

- clinical tests and treatments,

- self-management information,

- education or support,

- strategies for modifying health-related behaviours, managing stress, or solving practical problems.

A collaborative process in which patient and clinician discuss treatment or management goals (goal setting: see B below) and agree a plan for tackling these (action planning: see $\mathrm{C}$ below) are the essential features, but the full process may involve any of the following seven steps (see Figure 1):

Personalised care planning for adults with chronic or long-term health conditions (Review) 
Figure I. Conceptual model for the review

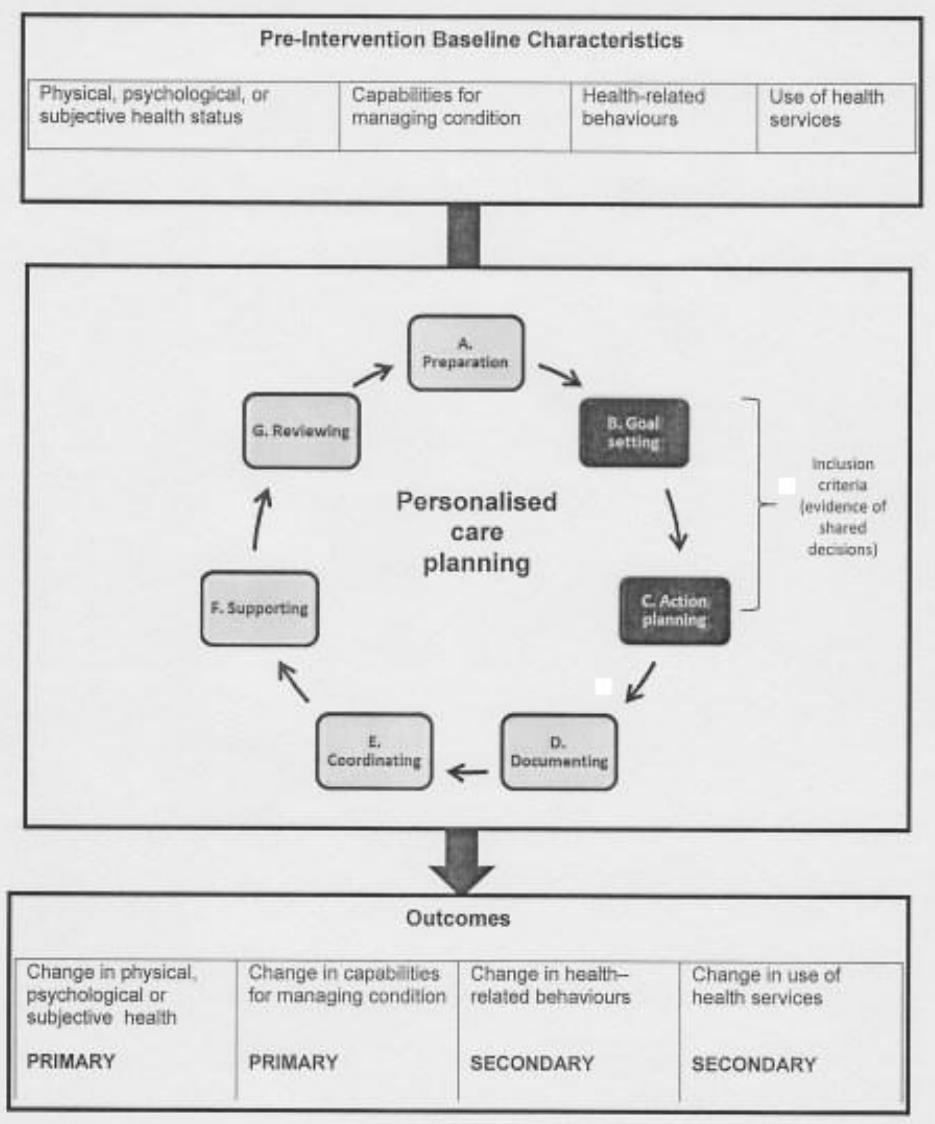

Possible Effect Modifiers (for subgroup analysis)

\begin{tabular}{|l|l|}
\hline Multi-morbidity & Heaith literacy \\
\hline
\end{tabular}

Personalised care planning for adults with chronic or long-term health conditions (Review)

Copyright $\odot 2015$ The Cochrane Collaboration. Published by John Wiley \& Sons, Ltd. 
- A. Preparation: the patient may be invited to a preliminary appointment to check their progress and undergo relevant clinical tests. Information (printed, electronic, written, or verbal) may be provided before or during the care planning appointment, to encourage the person to reflect on his or her condition and situation. This might include test results, and information about treatment options, or about health-related behaviours such as diet, exercise or smoking.

- B. Goal setting: aimed at agreeing treatment or management goals, the goal-setting process involves eliciting and clarifying patients' understanding of their condition, their values, outcome preferences and priorities. Patients may be encouraged to talk about their experience of living with the condition, their beliefs and concerns, and their comprehension of, and reactions to, the information provided. The discussion of what matters to them may cover treatment or management options, desired outcomes, lifestyle or behaviour changes, practical, social and emotional challenges, and problem-solving strategies. In personalised care planning, patients have scope to influence the agenda for discussion, and the choice of goals and priorities is not restricted to a prespecified list of professionally determined options.

- C. Action planning: a plan is jointly developed for working towards agreed goals. This may include identifying practical ways in which the patient can achieve their behavioural goals (for example, how and when to take more exercise), referring the patient to external sources of support, either within formal health services (for example, health coaching or rehabilitation services) or in the community (for example, exercise or cookery classes), or peer support. The plan may also include clinicianordered tests or treatments, referral to other clinical specialists or professionals, educational materials or courses, access to aids or appliances, care assistance or domestic help.

- D. Documenting: the agreed actions are usually documented in a specially-designed record (printed, electronic, or written) for use by the clinician(s) involved in the patient's care or for use by the patient as an aide-mémoire, or for both. These may be either a single shared record, or two separate records containing appropriate detail for clinician or patient.

- E. Co-ordinating: the clinician ensures that all tests, treatments, interventions, education, or support packages agreed in the action plan are available to the patient and provided in a well-co-ordinated fashion. This may include input from multidisciplinary team members, from hospital- or community-based specialists, from educationalists and other staff, or from community organisations or support groups.

- F. Supporting: patient and clinician agree a schedule for regular, systematic follow-up that may involve a number of contacts (face-to-face, telephone, or electronic) to provide appropriate support to help the patient solve problems and achieve his or her goals. This might take the form of health coaching, motivational support, problem solving, or simply checking and reinforcing progress in implementing the agreed plan.

- G. Reviewing: a meeting (face-to-face or remote) during which patient and clinician jointly review progress and plan next steps.

\section{How the intervention might work}

Personalised care planning aims to ensure that people receive appropriate support for self-managing their condition alongside any necessary clinical treatments from health professionals. The principles of self management have been developed in a number of theoretical models, mostly from the fields of psychology and behavioural science. They focus on understanding the factors that shape behaviour and those that might help people make the necessary adaptations to improve their health and ability to cope with illness and disability. Of these, Bandura's Social Cognitive Theory (Bandura 1977), Prochaska and DiClemente's 'Stages of Change' trans-theoretical model (Prochaska 1992), and Leventhal's SelfRegulation Theory (Leventhal 1998) are most often referred to. Taken together, these point to the importance of a sense of control or empowerment that can give people the confidence and motivation to take on and persist with new and difficult tasks. Interventions focus on confidence building and equipping individuals with the knowledge and skills to set personal goals and develop effective problem-solving strategies.

A commitment by both clinician and patient to shared decision making is considered essential for personalised care planning. The process is unlikely to succeed if either party is reluctant to participate. In shared decision making, health professionals and patients work together to understand problems, preferred goals and outcomes, sharing information and identifying options with the aim of reaching mutual agreement on the best course of action for the individual patient (Charles 1999; Elwyn 2012b; Entwistle 2012; Glasgow 2005b; Mulley 2012). This approach recognises explicitly that it is usually appropriate to enable people to make decisions about their care, ensuring they are well informed and well supported in the process of deliberation and decision making. Shared decision making takes as its starting point the notion that two types of expertise should be involved in selecting treatment or management options. Clinicians' expertise is based primarily on knowledge of the diagnosis, likely prognosis, treatment and support options, and the range of possible outcomes based on research evidence and population data; patients usually know more about the impact of the condition on their daily life, their personal values, preferences and attitude to risk, and the constraints they may face in implementing any recommended behaviour changes. 
Both types of knowledge are needed to manage illness successfully, so both parties should be prepared to share information and take decisions jointly.

This concept (shared decision making) has often been applied to 'acute' or 'elective' situations where there are choices between discrete interventions that are professionally controlled (for example, choice between a prescribed medicine or surgery, or choice about whether or not to have a 'preference-sensitive' screening test that only licensed professionals can administer), but it is also central to personalised care planning for long-term conditions when health professionals work with patients to determine goals and priorities (Bodenheimer 2003; Tsai 2005). Effective management of chronic conditions usually involves both tests and treatments prescribed by clinicians and actions that individuals must do for themselves, such as administering medication appropriately, or making lifestyle changes. In some cases, a patient may be better informed about their condition than the clinician, in which case the clinician should respect this expertise and take account of it in the planning process. Some patients may not need support for self management or behaviour change, but, for those that do, collaboratively-set goals and self-selected behavioural targets are seen as more motivational than clinician-assigned goals (Michie 2003). The process involves both shared decisions about how best to manage the condition, and shared responsibilities for implementing mutually agreed actions (Montori 2006). The experience of working together in a collaborative manner may lead to improvements in people's sense of confidence and well-being. Interventions to promote collaborative goal setting and action planning might, for example, make someone with a long-term condition feel respected, cared about, encouraged and capable of making a meaningful contribution to their state of health (Entwistle 2013).

\section{Why it is important to do this review}

Despite widespread support for the principle of personalised care planning, the nature and extent of evidence in support of this approach is unclear. The model has been promoted by the World Health Organization (WHO) and encouraged in a number of countries including Australia, the UK and USA (Singh 2008), but international surveys show that many people with long-term conditions do not receive sufficient support from health professionals to enable them to plan their care and self-manage their condition(s) effectively (Schoen 2011). For example, it has been government policy in England since 2010 to ensure that all people with long-term conditions are involved in a care-planning process (Department of Health 2009). This commitment has recently been strengthened by inclusion of an explicit promise in the $\mathrm{Na}$ tional Health Service Mandate that "everyone with long term conditions, including people with mental health problems, will be offered a personalised care plan that reflects their preferences and agreed decisions"(Department of Health 2012). In certain cases people with complex conditions or combinations of conditions may be offered a personal health budget to cover the costs of needs identified during the care planning process (Forder 2012). However, a co-ordinated, personalised approach is not yet the norm in everyday practice. While most people with long-term conditions in England report having some sort of care-planning discussions with clinicians in primary care, only a small proportion experience proactive, systematic support along the lines described above (Burt 2012; Newbould 2012).

Implementing care planning in primary care involves significant organisational and cultural change (Year of Care 2011). Health professionals may be reluctant to embark on this if they do not believe it is warranted by the evidence (Blakeman 2006). They may also be unwilling to adopt this approach if they feel it will be too time-consuming for them or too burdensome for their patients (Coulter 2011). There is a need for more information about which components of care planning are necessary and which may not be, and which types of tools or interventions are helpful (Burt 2013). For example, when it is important to complete the cycle of support and review, and when it might be sufficient to engage patients in goal setting and action planning only. Interventions specially designed for patients, clinicians or both may help to overcome barriers to implementation (see Types of interventions below).

People with multiple co-morbidities or cognitive impairments may find participation in care planning and self management especially difficult (May 2009).There are also concerns that this approach could exacerbate health inequalities if people with low levels of health literacy or communication difficulties are seen as less able to participate or lacking the capacity to self-manage their health (Coulter 2011).

Several systematic reviews have pointed to the importance of a patient-centred, personalised approach to care management. Patient-oriented interventions to support self management (for example, information provision or educational programmes) have led to improvements in health outcomes for people with diabetes (Deakin 2005; Renders 2000), asthma (Powell 2002) and a number of other chronic conditions (Foster 2007; Murray 2005). Various strategies for increasing people's motivation to adopt healthy behaviours (for example, motivational interviewing or use of written contracts) have led to improved health outcomes for some patients (Bosch-Capblanch 2007; Lai 2010; Rubak 2005; Smedslund 2011). Interventions designed to improve communications and encourage greater patient involvement in decision making have been shown to improve people's knowledge of screening or treatment options, but effects on health outcomes have been mixed (Dwamena 2012; Edwards 2013; Kinnersley 2007; Legare 2014; Levack 2012 (full review in press); Stacey 2014; Wetzels 2007). There is some overlap of focus between this latter group of reviews and the current one, in that they all cover strategies for engaging patients in decisions about their care, but none of the earlier reviews looked specifically at the effects of personalised care planning for people with long-term conditions. 


\section{O B J E C T I V E S}

To assess the effects of personalised care planning for adults with long-term health conditions compared to usual care (i.e. forms of care in which the active involvement of patients in treatment and management decisions is not explicitly attempted or achieved).

We addressed the following primary research questions:

- is personalised care planning effective for improving physical health (e.g. lipid measurements)?

- is personalised care planning effective for improving psychological health (e.g. anxiety and depression)?

- is personalised care planning effective for improving subjective health status (or health-related quality of life)?

- is personalised care planning effective for improving people's capabilities for self-managing their condition?

We also looked for evidence to address the following secondary research questions:

- is personalised care planning effective for improving people's health-related behaviours?

- how does personalised care planning impact on rates of use and costs of formal health services?

- what is the relative effectiveness of different types of intervention used to promote personalised care planning?

\section{METHODS}

\section{Criteria for considering studies for this review}

\section{Types of studies}

Randomised controlled trials and cluster-randomised controlled trials only.

\section{Types of participants}

We were interested in the ways that healthcare professionals and health services engage people in personalised care planning relating to chronic or long-term conditions. Chronic conditions are defined as "diseases of long duration and generally slow progression" (World Health Organization 2012): for example, heart disease and stroke, cancers, respiratory conditions such as asthma and chronic obstructive pulmonary disease (COPD), diabetes, kidney or liver disease, chronic pain and arthritis, neurological conditions such as epilepsy and multiple sclerosis, HIV/AIDS, and psychiatric conditions such as bipolar, schizophrenia or chronic depression.

We searched for studies where the participants were adults (aged 18 or over) with any long-term physical, psychological, sensory, or cognitive condition or combination of conditions affecting their health, treated in any setting (primary care, secondary care, community care or residential care). This could include people with long-term disabilities not necessarily caused by disease, such as blindness, deafness, mobility, communication problems or intellectual disabilities, if they are receiving treatment from health professionals.

We excluded studies involving simulated patients, or patients requiring treatment for acute or self-limiting problems only.

\section{Types of interventions}

The review examined trials that evaluated interventions (including changes to practice styles) that explicitly engaged patients in a shared decision-making process involving both goal setting and action planning as described in Description of the intervention above (B, C).

We excluded studies in which the intervention did not explicitly engage participants actively in determining their goals or priorities and developing a treatment/care/support plan, and those in which they were not encouraged to exert meaningful influence on goals and plans, or where their choices were unduly constrained. We also excluded studies that focused solely on group education programmes without one-to-one clinical engagement, and those designed primarily to engage people in making plans for end-oflife care (advance directives).

Various interventions or practices have been developed to encourage or support personalised care planning. These may be targeted at patients, clinicians or both, and may be used singly or in combination. Examples include the following:

\section{Patient-focused interventions:}

- information materials or decision aids for patients (Protheroe 2010)

- computer-based interventions to help patients identify and achieve behavioural goals (Glasgow 2004)

- suggested lists of questions the patient can ask to prompt the clinician to involve them more actively in decisions about their care (Shepherd 2011)

- health coaching and motivational support to help patients clarify objectives, solve problems and achieve behavioural goals (Frosch 2011)

- patient-held records for summarising personal goals and test results (Dijkstra 2005) 


\section{Clinician-focused interventions:}

- specific training programmes in shared decision making, care planning and/or motivational interviewing (Kennedy 2005)

- guidelines and feedback emphasising the need to elicit patients' preferences during care-planning consultations (Wensing 2003)

- algorithms embedded in clinical record systems to guide the care-planning process (Ell 2010)

\section{Interventions designed to influence the behaviour of both clinicians and patients:}

- brief tools for use within care-planning consultations to guide the discussion about options and agreed actions (Elwyn 2012a)

- an electronic or printed template for documenting jointlyagreed actions for use in monitoring and follow-up (Ross 2004).

Not all examples of these kinds of interventions met the review's inclusion criteria. We were primarily interested in the care-planning process itself. This could include any of the above-listed interventions, or others not described above. The point is that personalised care planning should cover whatever is required to help individual patients identify and achieve their own condition-related goals. In some cases both parties may conclude that the patient is managing well and that no additional medical intervention or support is needed.

Our focus was on patient engagement to support and enhance self management of long-term conditions in clinical settings. We excluded studies in which personalised care planning was not a major focus of the evaluation, or where it was not possible to isolate the specific effects of the personalised care planning process. We originally planned to compare the following types of intervention:

- personalised care planning (as defined above) compared to forms of care where individual involvement in treatment or management decisions is not explicitly encouraged (usual care)

- 'limited' approaches involving goal setting, action planning and no more than two additional steps (preparation, documenting, co-ordinating, supporting, reviewing: see Figure 1) versus 'extended' approaches involving five or more steps in the care-planning cycle

- patient-focused interventions versus those that aimed to change both patient and clinician behaviours

In the event we found even greater diversity among the interventions than predicted, so we added the following comparisons to tease out the likely effects of attributes such as the intensity of the intervention and whether it was integrated into the practice of the patient's usual care provider or an add-on service:

- intensity of intervention (high = at least one contact per month for more than three months; low = shorter duration and fewer contacts)
- integration into usual care (high = usual-care clinician involved in care planning and informed about patients' goals and plans; low = usual clinician not involved, not informed or both).

\section{Types of outcome measures}

See Figure 1 for an outline of the conceptual model used in the review, showing primary and secondary outcomes and subgroups. We focused on two main primary outcomes and two secondary outcomes, each of which included a number of potential measures.

\section{Primary outcomes}

1. Changes in health and well-being, including each of the following three dimensions measured separately:

i) physical health: measured instrumentally (e.g. blood pressure, blood lipids, body mass index, HbAlc, urinary albumin, etc.) or by observation or self report (including symptom scales, pain scores).

ii) psychological health: observation or self-report scales (e.g. depression or anxiety scores).

iii) subjective health status: patient-reported scales (including health-related quality of life, fatigue, self esteem, coping, activities of daily living, etc.) or proxy reports (clinicians' observations or family member/carer reports).

2. Changes in patients' self-management capabilities or indicators relevant to those capabilities: measured by self reports or observations (knowledge of their condition and its treatment or management options, self efficacy, activation, confidence or perceived competence, and ability to access relevant support). We included validated measures where possible. Non-validated measures were recorded but excluded from the meta-analysis.

\section{Secondary outcomes}

1. Changes in health-related behaviours: diet, exercise, smoking, use of relaxation techniques, self-management actions, condition-relevant self monitoring, adherence to treatment recommendations, attainment of personal goals.

2. Changes in use of formal health services: number and length of hospital admissions, number of outpatient, emergency department, or primary care visits, and, where recorded, effects on the costs of care.

We also recorded any reports of harms or adverse events associated with personalised care planning.

\section{Timing of outcome assessment}

We originally intended to group the outcomes into short-term (three months or less), medium-term (six to 12 months) and longterm (more than one year), but this proved difficult to do given the relatively small number of studies, so we have reported only the 
final outcome measures in each study and pooled these wherever possible.

\section{Selecting outcome measures for use in the analysis}

The outcomes listed above are broad categories. In the case of studies that reported more than one outcome within each of these groupings, we adopted the following process: two review authors (AC, AE or SR) independently listed outcomes (without considering either the size of the effect or its statistical significance). Many of the outcome measures used standardised self-completion questionnaires to obtain patients' reports. We pooled outcome data from studies that examined the same constructs, even if the measures were slightly different. Those that looked at different constructs or measured these in very different ways we reported narratively but did not include them in the meta-analysis.

\section{Search methods for identification of studies}

\section{Electronic searches}

In July 2013 we searched the following databases for all years:

- Cochrane Central Register of Controlled Trials

(CENTRAL), The Cochrane Library (July 2013, Issue 7).

- Dissertations \& Theses (ProQuest) (1743 - July 2013)

- MEDLINE \& MEDLINE In-process (Ovid) (1946 to July 2013)

- EMBASE (Ovid) (1974 to July 2013)

- PsycINFO (Ovid) (1967 to July 2013)

- Trial registers (clinicaltrials.gov) (21st June 2013)

- WHO International Clinical Trials Registry Platform (June 2013)

The search strategy was tailored to each of these databases and is reported in Appendix 1. It includes a list of terms developed by the Cochrane Consumers and Communication Review Group that covers most long-term conditions. There were no language restrictions.

\section{Searching other resources}

We scanned reference lists of relevant retrieved articles and reviews on this topic, to identify additional papers reporting results from the same study and relevant studies not identified by the electronic searches. We did not systematically search grey literature, conduct handsearches or contact experts. We included relevant studies irrespective of publication status.

\section{Data collection and analysis}

\section{Selection of studies}

We merged search results using EndNote software, and removed duplicates. Two of three review authors (AC, AE and SR) screened titles and abstracts independently to exclude clearly irrelevant references. Where, in the opinion of at least one review author, the abstract indicated that the study might be eligible for inclusion, or where it was not clear that the study should be excluded, we obtained full-text versions. We linked multiple reports of the same study.

We developed a standard form to record details of each study and reasons for inclusion or exclusion, based on the checklist below. Two review authors (AC, AE or SR) independently scrutinised all identified trial reports to determine eligibility, and recorded the reasons for including or excluding a study, which are documented in a PRISMA flow chart (Figure 2) and in the table Characteristics of included studies. After reviewing all relevant papers independently, the two authors compared notes and discussed any discrepancies. In cases where there was disagreement about eligibility, we referred papers to one of the review authors not involved in the initial selection process (VE, SS or RP).

We used the following checklist to determine eligibility:

1. Does the paper present primary data? EXCLUDE if review article, commentary, protocol, etc., but flag for later reference scan.

2. Was this a randomised controlled trial (RCT) or clusterrandomised trial (C-RCT)? EXCLUDE if not RCT or C-RCT, but flag for later reference scan to check for eligible studies not previously identified.

3. Did the study include adults aged over 18? EXCLUDE if all participants were children or young people aged under 18 . INCLUDE if age not stated or if participants included a majority of adults.

4. Did participants have one or more chronic conditions? EXCLUDE if participants were healthy people or simulated patients or were consulting for acute (time-limited) conditions.

5. Was the intervention concerned solely with planning for end-of-life care (advance directives)? If so, EXCLUDE.

6. Was the intervention a patient decision aid only, without one-to-one personalised care planning? If so, EXCLUDE.

7. Was the intervention patient education only, without oneto-one personalised care planning? If so, EXCLUDE.

8. Was the intervention a psychological treatment only, without one-to-one personalised care planning? If so, EXCLUDE.

9. Was personalised care planning with active involvement of the patients in a collaborative or shared decision-making process an explicit component of the intervention?

i) Were patients actively involved in planning their treatment or care with a clinician(s), coach or community health 
worker? INCLUDE IF THIS AND OTHER INCLUSION CRITERIA LISTED BELOW ARE MET

ii) Did the intervention include both collaborative goal setting and collaborative action planning? INCLUDE.

iii) Did trial include patient-based outcomes? If not, EXCLUDE; for example if outcomes related to clinicians only. Trials of training programmes for clinicians that included measures of their effects on patients should be considered for inclusion in the review if the training covered personalised care planning.

iv) Were patients encouraged to set their own goals or priorities and/or were they offered a choice of treatment or support package? INCLUDE if the intention of the intervention was to enable patients to have meaningful influence on goal selection and/or choice of treatment or support package. EXCLUDE if choices were constrained to only a few predetermined options, for example, only a choice between treatment $\mathrm{A}$ or treatment $\mathrm{B}$.

v) Was the care/action plan pre-prepared so patients had no opportunity to influence it? EXCLUDE.

vi) Was the care/action plan simply a pre-prepared list of instructions about what to do in particular circumstances? EXCLUDE.

vii) Is there any other evidence to suggest that the careplanning process did not allow the patient to be involved or to influence it? EXCLUDE

We recorded and reported all studies excluded for any of the reasons listed in criterion $9(\mathrm{a}-\mathrm{g})$ above (Characteristics of excluded studies). Studies excluded for any of the reasons itemised in 1 8 above have not been included in this table, but the numbers in each category are reported in Figure 2.

We collected and report below (Characteristics of ongoing studies) the details (citation details and other relevant information) of ongoing studies.

\section{Data extraction and management}

Two of three review authors (AC, AE and SR) independently extracted study characteristics and outcomes from reports.

We used a modified version of the template developed by the Cochrane Consumers and Communication Review Group to extract data from eligible studies (Characteristics of included studies).

\section{Assessment of risk of bias in included studies}

We assessed and reported the methodological risk of bias of included studies in accordance with the Cochrane Handbook for Systematic reviews of Interventions (Higgins 2011) and the guidelines of the Cochrane Consumers and Communication Review Group (Ryan 2011), which recommend explicit reporting of the following individual elements for RCTs: random sequence generation; allocation concealment; blinding of participants and personnel; blinding of outcome assessment; completeness of outcome data, selective reporting; and any other obvious sources of bias, such as comparability between groups at baseline or the possibility of contamination between the groups.

For cluster-RCTs we also assessed and reported the risk of bias associated with an additional domain: selective recruitment of cluster participants (Ryan 2011).

In all cases, two review authors extracted data and independently assessed the risk of bias of included studies, with any disagreements resolved by discussion and consensus. We contacted study authors for additional information or for clarification of the study methods, as required. We assessed the risk of bias in respect of random sequence generation. We made provision to exclude studies where this was assessed as high, but in fact we identified no such problems. We included all studies meeting the inclusion criteria in the review regardless of the assessment of risk of bias, but we conducted a sensitivity analysis (by excluding the study) if risk of bias due to method of randomisation or allocation concealment was unclear. The results of the 'Risk of bias' assessment were incorporated into the review through standard tables and narrative commentary, leading to an overall assessment of the risk of bias of the included studies and a judgement about the internal validity of the review's results.

\section{Measures of treatment effect}

We calculated effect sizes using mean difference (MD) with $95 \%$ confidence intervals (CI) in cases where studies had used the same measure (e.g. HbA1c). For most other outcomes, for example those using a variety of standardised questionnaires or patientreported outcome measures (PROMS), we used a standardised mean difference (SMD) with $95 \%$ CI to summarise the pooled effect of comparable outcomes. We used risk ratios (RR) and $95 \%$ CIs for dichotomous outcomes, where relevant, or transformed and treated them as continuous, and summarised them with the rest of the studies (based on the transformation of an odds ratio created from the equivalent two-by-two table). We did not backtransform them due to the variety of scales used in the studies and lack of consensus on which are the most appropriate.

\section{Unit of analysis issues}

Inclusion of cluster-randomised trials leads to potential unit of analysis problems. Whenever an adjusted (for clustering) effect was reported, we extracted this for inclusion in the review. No cluster trials reported analyses without adjusting for clustering and hence no further adjustment was necessary.

\section{Dealing with missing data}

We used intention-to-treat data in our analyses whenever possible. In cases where data were insufficiently reported in the published 
paper, we contacted the original authors for clarification and further information. Many studies reported baseline and endpoint measures which we used to calculate mean change and standard deviation. When available, we estimated the correlation coefficient for the baseline-endpoint values based on alternative studies. When this was not available, we used a correlation value of 0.5 instead (Follmann 1992).

\section{Assessment of heterogeneity}

Where studies were considered similar enough (based on consideration of diagnostic categories, type of intervention, outcome measures, or population subgroups) to allow pooling of data using meta-analysis, we assessed the degree of heterogeneity by visual inspection of forest plots and by examining the $\mathrm{Chi}^{2}$ test for heterogeneity. We quantified heterogeneity using the $\mathrm{I}^{2}$ statistic. An $\mathrm{I}^{2}$ value of $70 \%$ or more is taken as representing substantial levels of heterogeneity, but this value has to be interpreted in the light of size and direction of effect and strength of evidence for heterogeneity, based on the $\mathrm{P}$ value from the $\mathrm{Chi}^{2}$ test (Higgins 2011). We did not report pooled results where we detected substantial clinical, methodological or statistical heterogeneity across included studies. We assessed possible clinical or methodological reasons for any variation by grouping studies that were similar in terms of diagnostic categories, intervention types or population subgroups to explore differences in intervention effects.

\section{Assessment of reporting biases}

We have not assessed publication bias by use of funnel plots because we had too few studies to do so. Instead we assessed reporting bias qualitatively by looking at the properties of the included studies (for example, if only small studies with positive findings were identified for inclusion, or where authors indicated that there were relevant unpublished studies).

\section{Data synthesis}

We pooled data using a fixed-effect meta-analysis because of the small number of studies. In the absence of unit of analysis errors, we combined data from individual and cluster-randomised controlled trials.

\section{Subgroup analysis and investigation of heterogeneity}

We aimed to analyse results for the following subgroups to examine factors that might modify any effects (see Figure 1):

- multi-morbidity: people with multiple (i.e. more than one) chronic conditions or disabilities. We considered depression associated with another condition such as diabetes a comorbidity, rather than an example of multi-morbidity.
- health literacy: people who face communication or comprehension problems due to low educational level, minority language, cognitive impairment or intellectual disability.

In practice we were unable to do this due to a paucity of studies measuring these issues, so we have reported any relevant results in the narrative only.

\section{Sensitivity analysis}

We used sensitivity analyses to determine the impact of our choices and assumptions. We explored the impact of the inclusion of high/ low quality studies in the review (see Assessment of risk of bias in included studies above).

\section{'Summary of findings' table}

We prepared a 'Summary of findings' table based on the methods described in chapter 11 of the Cochrane Handbook for Systematic Reviews of Interventions (Schünemann 2011). We have presented the results for the major comparisons of the review (personalised care planning versus usual care) for each of the primary outcomes (physical health, psychological health, subjective health status, and self-management capabilities), as outlined in the Types of outcome measures section. We have provided a source and rationale for each assumed risk cited in the tables, and have used the GRADE system to rank the quality of the evidence (Schünemann 2011).

\section{Consumer participation}

We recruited an expert patient advisory group of six people with experience of living with long-term conditions. Between them they had experience of living with the following conditions: Alzheimer's disease (carer of family member), anxiety, asthma, bilateral aboveknee amputation, cataracts, depression, epilepsy, erythromelalgia, irritable bowel syndrome, labyrinthitis, migraine, multiple sclerosis, myeloproliferative disorder, over- and under-active thyroid, peripheral vascular disease, polycystic ovaries, poor circulation, Raynaud's syndrome, rheumatoid arthritis, and tendonitis. They agreed to advise on various aspects of the review, including the protocol, selection of outcome measures, and emerging findings. They were paid a fee for their time.

We sought input and advice from the expert patient advisory group via a secure dedicated website where they could record comments and queries. The website included a short summary of the research plan as background information. We encouraged group members to submit questions about the study at any time via the website. During the development of the protocol we asked them to review the outcomes we had selected for the study. We asked them to indicate which of these should have highest priority in the light of their own experience, to rank all other outcome measures in order of priority and to give reasons for their ranking. We also asked them 
to let us know if any important outcomes were missing. Participants provided detailed and helpful comments on their rankings. They acknowledged the need to reduce the number of outcomes to make the review manageable, but at least one participant indicated discomfort with this procedure which they felt smacked of standardisation rather than personalisation, giving a professional rather than patient focus to the review. Nevertheless, the results of this exercise supported the choice of outcomes listed above, and no outcomes of any significance were identified as missing from the review.

We asked the group to give their reactions to the findings of the review and to assess the plain language summary to ensure it was comprehensible, accessible and relevant. The group has also been asked to help with disseminating the findings from the completed review.

\section{R E S U L T S}

\section{Description of studies}

We restricted the search to randomised controlled trials and cluster-randomised trials evaluating interventions that focused on or included personalised care planning.

\section{Results of the search}

The electronic search yielded 16,151 records. We added a further 12 studies identified in reference scans. Following removal of duplicates, we screened a total of 9890 unique abstracts for eligibility and excluded 9494 of these. We obtained full-text articles for the remaining 396 abstracts and assessed these for inclusion in the review. We excluded a total of 353 articles following full-text analysis. We have listed below (Characteristics of excluded studies) only those studies where we had to involve a third review author to resolve any uncertainties or differences in the assessments of the first two review authors. We deemed a total of 43 articles eligible for inclusion. These described results from 19 unique studies and seven protocols (Figure 2). We tried to contact the authors of eight of the studies to ask for further information or unpublished data but could not track down current contact details for two. We received helpful replies from five lead authors (Battersby 2007; Ludman 2007; Naik 2011; Stanhope 2013; Wilson 2010). 
Figure 2. Study flow diagram.

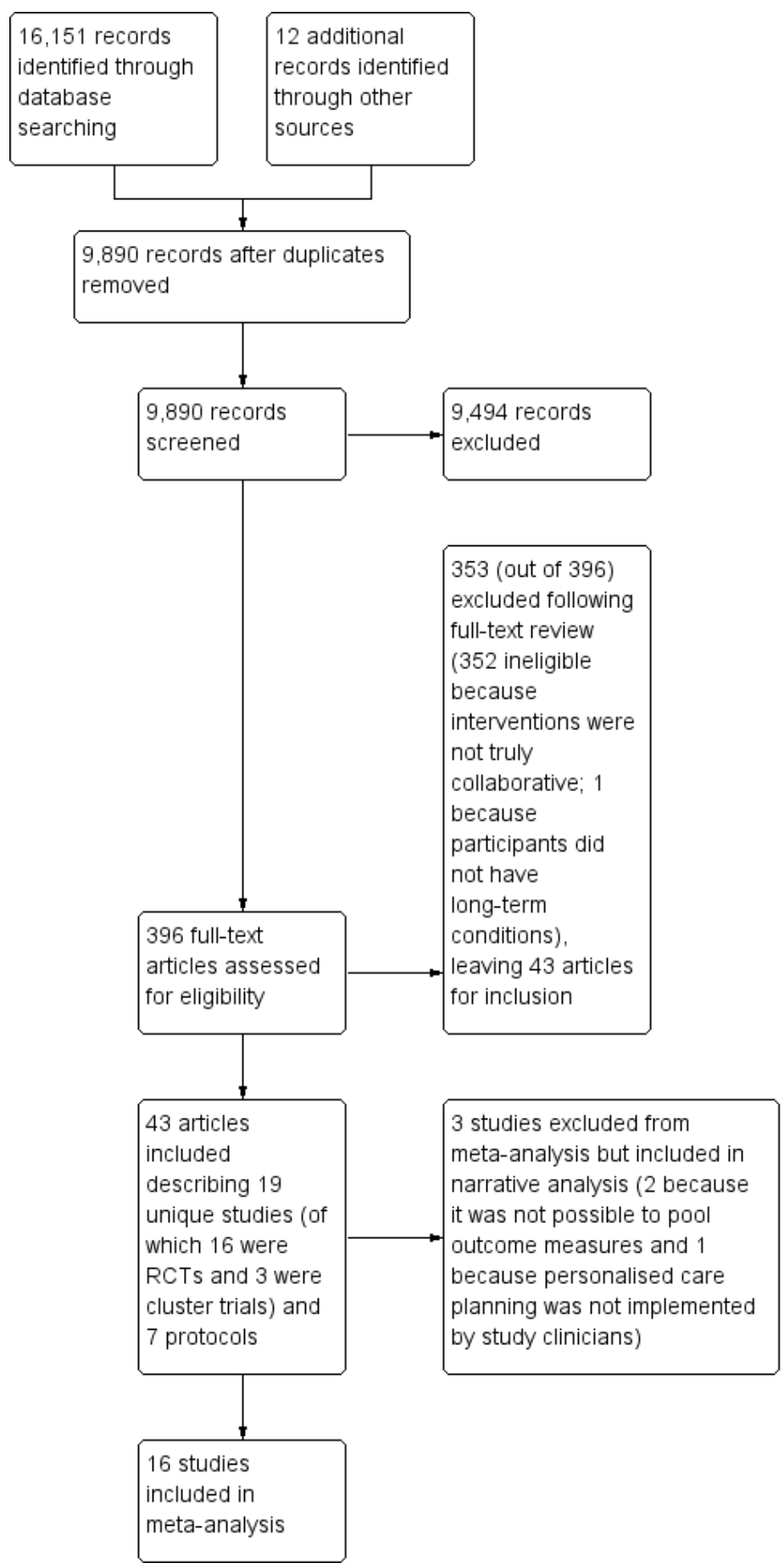

Personalised care planning for adults with chronic or long-term health conditions (Review) 


\section{Included studies}

The 19 completed studies included 16 randomised controlled trials (RCTs) (Battersby 2007; Frosch 2011; Hart 1978; Hiss 2007; Katon 2010a; Liu 2012; Ludman 2007; Naik 2011; Schillinger 2009; Shearer 2007; Thom 2013; Tsay 2004; Van der Wulp 2012; Wilson 2010; Wolever 2010; Zoffmann 2006), and three cluster trials (Glasgow 2005a; Kennedy 2013; Stanhope 2013). Thirteen of the included studies were conducted in the USA and one each in Australia, China, Denmark, the Netherlands, Taiwan, and the UK. Details of the studies and the interventions are provided in the table of Characteristics of included studies and summarised in Table 1.

We were able to include 16 of the 19 studies in a meta-analysis. We had to exclude two studies because they used outcome measures that were unique to these studies so could not be pooled (Hart 1978; Stanhope 2013). A third study attempted to evaluate an intervention (a change in practice) that explicitly engaged patients in personalised care planning as we have defined it, but the intervention (the intended new style of practice) was not actually implemented (Kennedy 2013). The authors of this study carried out a process evaluation that confirmed this (Kennedy 2014). We therefore excluded it from the meta-analysis on the grounds that the study cannot tell us anything about the effects of engaging patients in care planning. The only outcome that this study shared in common with others in this review (and therefore could have been pooled) was the Stanford self-efficacy questionnaire. We took the view that inclusion of data from this study would have introduced a negative bias into the meta-analysis.

\section{Participants}

There was considerable variation in the size of the studies, ranging from 32 participants (Hart 1978) to 5599 (Kennedy 2013). Together they included a total of 10,856 participants (Table 1). For trials comparing three or more arms, we selected the study arm that most closely met our inclusion criteria, so the data included here represent a subset of those in the published papers for the following studies: Battersby 2007; Ludman 2007; Schillinger 2009; Wilson 2010.

Twelve studies focused on people with diabetes, with or without associated conditions (Frosch 2011; Glasgow 2005a; Hiss 2007; Katon 2010a; Kennedy 2013; Liu 2012; Naik 2011; Schillinger 2009; Thom 2013; Van der Wulp 2012; Wolever 2010; Zoffmann 2006), three focused on mental health (Hart 1978; Ludman 2007; Stanhope 2013), one on heart failure (Shearer 2007), one on endstage renal disease (Tsay 2004), one on asthma (Wilson 2010), and one on various conditions (Battersby 2007). This last study included eight sub-studies in four different regions in South Aus- tralia, half of which were separate but linked RCTs using similar methods and measures (the other four sub-studies used geographic controls so were ineligible for inclusion). The four eligible trials focused on patients with cardiac conditions, respiratory conditions, somatisation and problems of old age.

Only one study used a formal assessment of health literacy: Schillinger 2009 assessed 59\% of their participants as having 'limited' literacy according to the Test of Functional Health Literacy in Adults (ToFHLA) scale.

Five studies had participants consisting mainly of people from lower socio-economic groups or from minority ethnic groups or from both (Frosch 2011; Hiss 2007; Kennedy 2013; Schillinger 2009; Thom 2013).

No study focused explicitly on patients with multi-morbidities. One study (Katon 2010a) included patients with depression with diabetes or coronary heart disease or both but, since depression is often a side effect of these conditions, we considered this a comorbidity rather than a study of the effects on people with multimorbidities.

\section{Interventions}

While all studies involved interventions that included personalised care planning (goal setting and action planning), there was considerable variation in the way this was carried out and in the tools and techniques adopted to support the process (Table 1).

All 19 studies included components that were intended to support behaviour change among patients, involving either face-toface support or telephone support. Three of the interventions took place in hospital clinics (Shearer 2007; Tsay 2004; Zoffmann 2006), the remainder in primary care or community settings. In most cases the intervention focused on changing patients' capabilities and behaviour (15 studies) but four studies (Battersby 2007; Kennedy 2013; Stanhope 2013; Wilson 2010) aimed to change the behaviour of both patients and clinicians.

A variety of tools and techniques were used in the interventions, including patient information packages (DVDs, computer programmes, or booklets); prompts for patients (patient-held records, worksheets or decision aids); structured consultations using coaching methods such as motivational interviewing; training or prompts for clinicians; peer support; and both individual and group visits (see Table 1).

In most cases (14 studies) the care-planning process was led by nurses, or nurses and therapists acting as care managers, service coordinators or health coaches. Doctors were actively involved in six of the studies (Battersby 2007; Hiss 2007; Katon 2010a; Kennedy 2013; Liu 2012; Naik 2011) including one study (Naik 2011) where physicians were solely responsible. In two studies the main contact was a peer coach (Thom 2013; Van der Wulp 2012) and 
in two the intervention was provided by mental health providers including social workers (Hart 1978; Stanhope 2013).

Only five studies relied solely on patients' usual-care clinicians to conduct the intervention (Table 1). In 10 studies the intervention involved contact with additional specially-trained staff or peers not usually responsible for the patient's care. Four studies involved both usual-care clinicians and additional clinicians. Contact between clinicians or peer coaches and patients was face-to-face in 15 studies, while the remaining four studies relied solely or mainly on telephone contact.

We grouped studies according to the number of completed stages in the care-planning cycle (Figure 1). Our prespecified inclusion criteria selected interventions that had completed at least two of the seven collaborative-planning stages (B - goal setting, C - action planning) and some form of follow-up support was included in all 19 studies ( $\mathrm{F}$ - supporting). Of the other stages, A - preparation for care planning (for example, preliminary information packages or sending test results to the patient so they could review these in advance of the consultation) formed part of the intervention in only four studies, D - documenting (i.e. a record that is explicitly shared with the patient) featured in seven studies, E - co-ordinating (i.e. the care manager liaising with clinicians and other staff to ensure that all issues identified were dealt with) was reported in five studies, and G - reviewing progress and making further plans was an explicit feature of only three studies. We classified those that had completed only three or four of these stages as 'limited', while those where the intervention involved five or more of the stages were classified as 'extended' (Table 2). Five studies fell into the 'extended' group and only two of these (Battersby 2007; Katon 2010a) covered the entire cycle (A - G).

We also classified interventions according to the intensity of the intervention and the extent to which they were integrated into clinical practice (Table 3). Where studies did not explicitly state that a particular process was carried out (for example, a stage in the care-planning cycle, a precise number of patient-clinician contacts, or co-ordination with usual care providers), we have assumed that these were not features of the intervention and have classified them as 'low' or 'no'. Eight studies fell into the high-intensity group and 11 were low-intensity. A different group of eight studies was classified as integrated with the patient's usual provider, while 11 were not. Only four studies were rated high on both these measures (Battersby 2007; Hiss 2007; Katon 2010a; Liu 2012).

Interventions varied in the extent to which the clinician input was standardised and supervised to ensure fidelity to the design (see Characteristics of included studies). Some studies used tightlycontrolled interventions involving closely-supervised clinicians, while others were more pragmatic in design. Reports from one study (Kennedy 2013) indicated that a majority of participant clinicians had not delivered the intervention as intended.

A theoretical framework can be useful for explaining how the intervention is expected to work, but this was mentioned in only nine of the 19 studies (see Characteristics of included studies). Five studies cited the Chronic Care Model (Battersby 2007; Glasgow 2005a; Kennedy 2013; Ludman 2007; Schillinger 2009), one mentioned Rogers's Science of Unitary Human Beings (Shearer 2007), one was based on Bandura's Social Cognitive Theory (Van der Wulp 2012), one cited Prochaska and di Clemente's Stages of Change theory (Zoffmann 2006) and one was a Chinese adaptation of the Stanford Chronic Disease Self-Management Programme (Liu 2012).

The comparison group was usual care in 12 of the studies. The remaining seven made a comparison between personalised care planning and various forms of enhanced usual care (Glasgow 2005a; Katon 2010a; Kennedy 2013; Naik 2011; Stanhope 2013; Thom 2013; Tsay 2004). Additions to usual care in these studies included provision of health information, group education, or enhanced access to primary care physicians and other clinical staff.

\section{Risk of bias in included studies}

Details of our judgements and the rationale for these are included in the Characteristics of included studies table and displayed in Figure 3 and Figure 4. 
Figure 3. Risk of bias graph: review authors' judgements about each risk of bias item presented as percentages across all included studies.

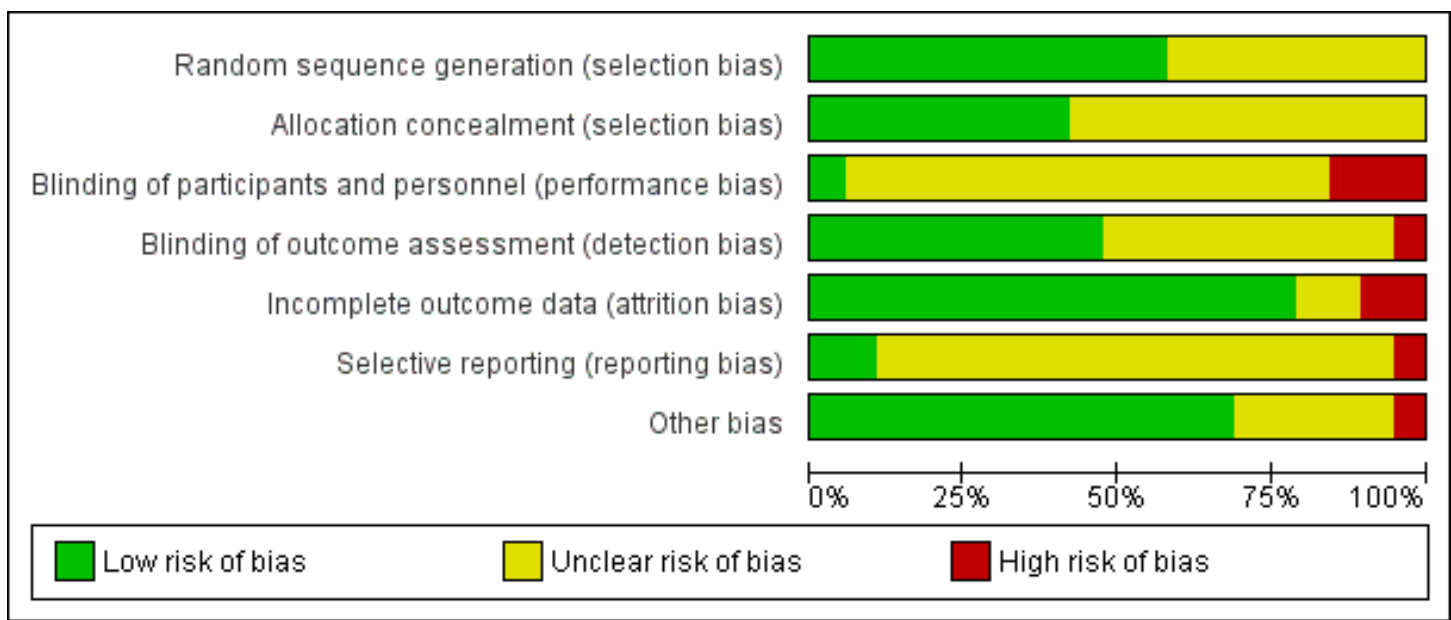


Figure 4. Risk of bias summary: review authors' judgements about each risk of bias item for each included study.

\begin{tabular}{|c|c|c|c|c|c|c|c|}
\hline & 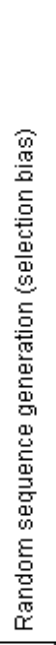 & 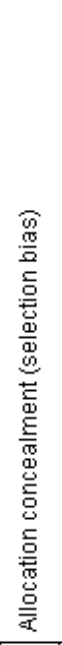 & 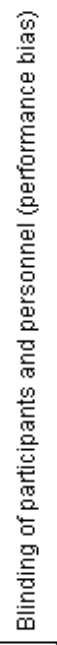 & 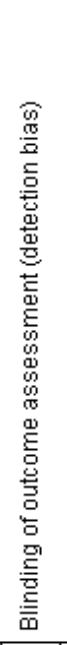 & 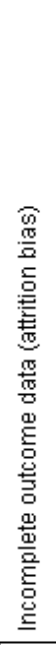 & 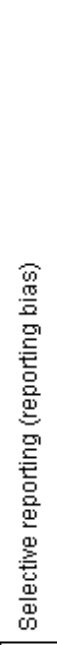 & 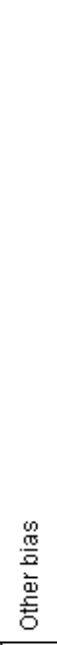 \\
\hline Battersby 2007 & + & + & $?$ & + & - & - & $?$ \\
\hline Frosch 2011 & $?$ & + & + & + & + & $?$ & + \\
\hline Glasgow 2005a & + & $?$ & $?$ & $?$ & + & $?$ & + \\
\hline Hart 1978 & $?$ & $?$ & $?$ & $?$ & $?$ & $?$ & + \\
\hline Hiss 2007 & $?$ & $?$ & $?$ & $?$ & + & $?$ & + \\
\hline Katon 2010a & + & + & $?$ & $?$ & + & + & + \\
\hline Kennedy 2013 & + & $?$ & ? & + & + & + & - \\
\hline Liu 2012 & + & $?$ & - & + & + & $?$ & $?$ \\
\hline Ludman 2007 & + & + & $?$ & + & + & $?$ & $?$ \\
\hline Naik 2011 & ? & + & ? & + & + & $?$ & + \\
\hline Schillinger 2009 & ? & $?$ & ? & + & + & $?$ & + \\
\hline Shearer 2007 & $?$ & $?$ & - & $?$ & - & $?$ & $?$ \\
\hline Stanhope 2013 & ? & $?$ & $?$ & - & $?$ & $?$ & $?$ \\
\hline Thom 2013 & + & $\odot$ & $?$ & $?$ & + & $?$ & + \\
\hline Tsay 2004 & + & $?$ & $?$ & + & + & $?$ & + \\
\hline Van der Wulp 2012 & + & + & $?$ & $?$ & + & $?$ & + \\
\hline Wilson 2010 & + & + & $?$ & + & + & $?$ & + \\
\hline Wolever 2010 & ? & $?$ & - & $?$ & + & $?$ & + \\
\hline Zoffmann 2006 & + & $?$ & $?$ & $?$ & + & $?$ & + \\
\hline
\end{tabular}




\section{Allocation}

Just over half of the studies (11 out of 19) reported an acceptable method of random sequence generation. Eight studies did not provide an adequate description of the randomisation process, so we classified these as unclear. Allocation concealment appeared satisfactory in eight of the studies, but it was inadequately described in 11 studies.

\section{Blinding}

Blinding of participants and personnel is almost impossible in this type of study, so we classified the risk as unclear for 16 of the studies, since these relied on objective clinical measurements (e.g. blood tests) and we did not consider the risk of non-blinding to be especially problematic. Detection bias was rated high for three studies (Liu 2012; Shearer 2007; Wolever 2010) where both participants and personnel were aware of treatment status and a number of outcomes were subjective. Blinding of outcome assessment was adequate in nine studies, but a further nine provided inadequate evidence. One study (Stanhope 2013) was classified as high risk because it relied on non-blinded clinicians' reports of medication adherence (its primary outcome) with no independent validation of this measure.

\section{Incomplete outcome data}

Most studies (15) were rated at low risk in respect of attrition bias, but two provided inadequate information and two were deemed to be at high risk: Battersby 2007 because of very high attrition rates (47\% loss to follow-up in the intervention group and 50\% in the control group) and Shearer 2007 because of a great deal of missing data: one or more dependent variable pretest scores were missing for $24 \%$ of the experimental group and $24 \%$ of the control group at baseline, and for $31 \%$ and $42 \%$, respectively, at post-test.

\section{Selective reporting}

We considered reporting bias to be at low risk in two studies where there were pre-published protocols, at unclear risk in 16 where we found no published protocols, and at high risk in one (Battersby 2007), which reported only statistically significant outcomes and not those that were non-significant. This study comprised a series of linked trials using similar methods, but only one of these substudies (Pols 2008) provided sufficient information for some outcome measures to be included in the meta-analysis. We excluded the other sub-studies because it proved impossible to obtain full results from the authors. We did not include outcome measures in the meta-analysis if full data were available for the intervention group only and not the control group.

\section{Other potential sources of bias}

We considered other potential sources of bias, such as selective recruitment and fidelity to the intervention. We classified 13 studies as at low risk on these criteria, five were unclear and one (Kennedy 2013) was rated at high risk due to the fact that the intervention was not implemented as intended. The authors reported poor fidelity on the part of clinicians: collaboration between clinicians and patients (shared decision making) at six months was significantly less in the intervention group than in the control group ( $\mathrm{P}$ $=0.05$ ); only $2 \%$ of patients with irritable bowel syndrome were referred to therapists as required in the protocol; and $42 \%$ of clinicians failed to use the PRISMS tool which was intended to help patients express their needs and preferences. A process evaluation (Kennedy 2014) confirmed this impression of very poor fidelity to the intervention.

\section{Effects of interventions}

\section{See: Summary of findings for the main comparison}

\section{Physical health}

Eleven studies examined the effects of personalised care planning on physical health using a variety of standardised clinical indicators, including glycated haemoglobin (HbAlc), blood pressure (systolic (SBP) and diastolic (DBP)), cholesterol (LDL-C), body mass index (BMI), lung function (FEV1) and asthma control (ATAQ) (Table 4). We pooled data from 10 of the 11 studies in at least one comparison, omitting one study from the meta-analysis (Wilson 2010) because it used unique measures (FEV1, ATAQ).

1. Glycated haemoglobin: (Analysis 1.1) Nine studies (1916 participants) measured $\mathrm{HbA} 1 \mathrm{c}$ at six or 12 months postintervention, giving a combined mean difference (MD) between intervention and control of $-0.24 \%, 95 \%$ confidence interval (CI) -0.35 to -0.14 , a small positive effect in favour of personalised care planning compared to usual care. Excluding studies with unclear randomisation method and allocation concealment made little difference (MD - $0.25 \%$, 95\% CI - 0.36 to -0.14$)$.

2. Systolic blood pressure: (Analysis 1.2): Six studies (1200 participants) measured SBP, giving a combined MD of -2.64 $\mathrm{mm} / \mathrm{Hg}, 95 \% \mathrm{CI}-4.47$ to -0.82 , a small positive effect in favour of personalised care. However, a sensitivity analysis to exclude studies at higher risk of bias reduced this to $-0.64 \mathrm{~mm} / \mathrm{Hg}, 95 \%$ CI -3.70 to 0.41 .

3. Diastolic blood pressure: (Analysis 1.3) the pooled results from four studies (751 participants) showed no effect on DBP, $\mathrm{MD}-0.71 \mathrm{~mm} / \mathrm{Hg}, 95 \% \mathrm{CI}-2.26$ to 0.84 .

4. Cholesterol: (Analysis 1.4) the pooled results from five studies (1545 participants) showed no statistically significant 
effect on LDL-C, standardised mean difference (SMD) $0.01 \mathrm{mg} /$ dL, $95 \%$ CI -0.09 to 0.11 .

5. Body mass index: (Analysis 1.5) the pooled results from four studies (822 participants) showed no effect on BMI, MD $0.11 \mathrm{~kg} / \mathrm{m}^{2}, 95 \% \mathrm{CI}-0.35$ to 0.13 .

6. Other: a single study of asthma patients (Wilson 2010) reported improvements associated with personalised care planning in lung function: adjusted mean FEV1 as a percentage of predicted value was $76.5 \%$ in the intervention group versus $73.1 \%$ in the control group, and in asthma control measured by the Asthma Therapy Assessment Questionnaire (ATAQ) (Juniper 1992): odds ratio (OR) of reporting no asthma control problems $1.9,95 \%$ CI 1.3 to 2.9 , in favour of personalised care.

\section{Psychological health}

Seven studies examined the effects of personalised care planning on psychological outcomes (Table 5). Where studies used more than one measure of psychological outcome, we selected the one that was most conceptually similar to the measures used in the other studies. We pooled results from those studies that used one of four standardised measures of depression symptoms: the Patient Health Questionnaire (PHQ-9) (Kroenke 2001), the Hopkins Symptom Checklist 20 (SCL-20) (Derogatis 1974), the Beck Depression Inventory (Beck 1988), and the Center for Epidemiologic Studies Depression Scale (CES-D) (Radloff 1977).

1. Depression: (Analysis 2.1) six studies measured depression using one of the above four measures at various time points postintervention. We were able to pool results from five of the studies (599 participants), giving a SMD of $-0.36,95 \%$ CI -0.52 to 0.20 , a small positive effect in favour of personalised care. However there was substantial heterogeneity in results from the individual studies. A sensitivity analysis to exclude studies at high risk of bias increased the effect to SMD $-0.55,95 \%$ CI -0.76 to 0.34 . The remaining study (Glasgow 2005a) reported the proportion of participants with major depression (score 10 or higher on PHQ-9) and found greater improvement in the control group than the intervention group (OR 2.94, 95\% CI 1.36 to $6.34 ; 886$ participants).

2. Other measures of psychological health: we excluded several other measures of psychological health from the pooled analysis, either because the study included another measure that seemed a better fit, or because the instrument measured a different concept, for example perceived stress or perceived benefits of the condition. Katon 2010a used the Patient Global Rating for Improvement (PGI) in depression measure (Guy 1976) and found that participants in the intervention arm were more likely to report an improvement in their depression symptoms $(41 / 92,45 \%)$ than those in the control arm (16/91, $18 \%)(<0.001)$. Ludman 2007, which was a pilot study for Katon 2010a, used the PGI measure but the sample size was too small to detect differences in outcomes. This study also included the Structured Clinical Interview for DSM-IV depression module (SCID) (First 1997), but again they found no effect due to the small sample size. Wolever 2010 used the Perceived Stress Scale (PSS-4) (Cohen 1983a) and the Benefit Finding Scale (Tomich 2004), and found improvements on both measures for the intervention group but not the control group.

\section{Subjective health status}

Ten studies used various patient-reported measures of health status (or quality of life), including both generic health status measures and condition-specific ones (Table 6).

1. Generic health status:Analysis 3.1 and Analysis 3.2: five studies used the SF-36 patient-reported health status measure (Ware 1992) or the briefer SF-12 (Ware 1996), which reproduces the eight-scale profile of the SF-36 with fewer levels, yielding less precise scores but based on the same constructs. The resulting profile is often presented as two summary scores: the physical component score (PCS) and the mental component score (MCS). Three of the included studies used this method, so we pooled the results from these three studies. The combined analysis from the three studies (345 participants) gave a SMD on the PCS of $0.16,95 \%$ CI -0.05 to 0.38 , and on the MCS of $0.07,95 \%$ CI -0.15 to 0.28 . It was not possible to include the remaining two studies in this meta-analysis because one (Kennedy 2013) used only selected sub-scales of the SF-36 and anyway was withdrawn from the meta-analysis for the reasons described above, and the other (Wolever 2010) gave a single score based on the SF-12 without the MCS/PCS breakdown. Neither of these two studies found a difference between the personalised care group and the usual care group post-intervention.

2. Other generic measures of health status:Battersby 2007 reported improvements in functioning as measured by the Work and Social Adjustment Scale (WSAS) (Mundt 2002) in all but one of their sub-regional trials. Katon 2010a used the Sheehan Social Role Disability scale (Leon 1997), the WHO Disability Assessment Schedule (WHODAS-2) (Ustun 2010), and a global rating scale. They found that participants in the personalised care arm experienced greater improvements at six and 12 months from baseline on both the Sheehan Disability scale (effect size $0.30, P=0.006$ ) and the global quality of life rating scale (effect size $0.39, \mathrm{P}=0.005$ ) than those in the usual care group. They found no difference between groups on WHODAS-2. Kennedy 2013 used the Euro-Qol measure (EQ-5D) (Kind 1996), but found no difference between the groups. We have not pooled these data as the measures differ from each other conceptually.

3. Condition-specific health status: (Analysis 3.3) four studies (1330 participants) included a questionnaire to measure condition-specific health status. In two cases this was the Problem Areas in Diabetes scale (PAID-2) (Welch 1997), one study used the Stanford Illness Intrusiveness scale (Devins 2010), and one the Asthma Quality of Life Questionnaire (AQLQ) 
(Juniper 1999). We considered that these scales were sufficiently similar to pool the data. The combined results showed no difference between the groups: SMD $-0.01,95 \%$ CI -0.11 to 0.10 , but this was characterised by heterogeneity between the studies.

\section{Self-management capabilities}

Nine studies looked at the effect of personalised care on capabilities related to aspects of self management using a variety of outcome measures (Table 7).

1. Self efficacy: (Analysis 4.1). Self efficacy refers to an individual's confidence to carry out necessary tasks or procedures to manage their health or health care. Eleven studies used instruments designed to measure self efficacy for health-related behaviours, seven of which reported improvements. After excluding Kennedy 2013 for the reasons stated above, we pooled results for five of the studies (471 participants) that used similar scales. These included the Stanford self-efficacy scales (Lorig 1996), a scale called Strategies Used by People to Promote Health (SUPPH) (Lev 1996), a Dutch scale referred to as the Diabetes Management Self-Efficacy Scale (Van der Bijl 1999), and the Perceived Competence in Diabetes Scale (PCDS) (Williams 1998) (Table 7). The combined results showed a positive effect of personalised care planning: SMD $0.25,95 \%$ CI 0.07 to 0.43 .

2. Other self-management capabilities: Self efficacy can be seen as a contributor to, or partial indicator of, capabilities, but its measurement is usually restricted to a limited sub-set of the capabilities for self management that people with long-term conditions value. A further five studies measured other attributes that contribute to self-management capabilities, including knowledge and understanding (the University of Michigan Diabetes Knowledge Test (Fitzgerald 1998); enablement and activation (the Patient Enablement Instrument (PEI)) (Howie 1998) and the Patient Activation Measure (PAM-13) (Hibbard 2005)); purposeful participation in attaining health goals (Power as Knowing Participation in Change Tool (PKPCT)) (Caroselli 1998); coping (Appraisal of Diabetes Scale (ADS)) (Carey 1990); empowerment (Diabetes Empowerment Scale (DES)) (Anderson 2000); and interpersonal support (Interpersonal Support Evaluation List - ISEL-12) (Cohen 1983b). We did not attempt to pool these data because they measured different constructs. The results from the individual studies were mixed (Table 7). The two studies that used PAM-13 (or parts of it) found evidence of an effect on patient activation: Wolever 2010 used the PAM-13 scale and found a statistically significant timeby-group interaction in favour of personalised care. This study also noted improvements in reported interpersonal support for the intervention group over the control group using the ISEL-12 scale. Katon 2010a found improvements among the intervention group in two of the four PAM questions they selected, and Tsay 2004 reported an effect on empowerment for the personalised care group compared to the control group, but Shearer 2007 found no effect in relation to the PKPCT.

\section{Adverse events}

Only one study (Katon 2010a) reported any harms: 27 participants in the intervention group and 23 in the control group were hospitalised during the course of the study; one person in the intervention group and two in the control group died. There were no differences between intervention and usual care groups and there is no reason to assume that these adverse events were due to the intervention.

\section{Secondary outcomes}

\section{Health-related behaviours}

Ten studies included measures of the effects of personalised care on health-related behaviours, including exercise, diet, medication adherence and self-care activities (Table 8).

1. Exercise: (Analysis 5.1) we were able to pool the results from six studies (907 participants) that included patients' self reports on exercise frequency, but found no effect: SMD 0.11, 95\% CI -0.02 to 0.24 .

2. Diet: four studies measured the effect on diet using various different self-report measures (Frosch 2011; Katon 2010a; Liu 2012; Van der Wulp 2012) which could not be pooled. None of these found a difference between the intervention and control groups.

3. Medication adherence: five studies measured the effect on medication adherence. Two of these presented patients' self reports on adherence, two gave pharmacy reports and one gave clinicians' reports. We were unable to pool these data because of the diversity of measures used. Frosch 2011 found no effect of personalised care on adherence. Katon 2010a found that patients' knowledge about their medicines and confidence to follow medical regimens improved, but adherence (pharmacy data) did not. Stanhope 2013 reported improvements in medication adherence (clinician report) among the intervention group but not for the control group. Wilson 2010 reported improvements for the intervention group on a number of different pharmacy-derived measures of adherence. Wolever 2010 found an improvement for the personalised care group over the control group on the ASK-20 adherence barrier questionnaire (Matza 2008).

4. Self-care activities: three of the five studies that measured the impact of personalised care planning on performance of selfcare activities (Katon 2010a; Schillinger 2009; Shearer 2007) found improvements in the personalised care group compared to the control group. We were unable to include Katon 2010a in the meta-analysis because of the way the results were reported (blood glucose monitoring mean 4.9 days per week in the intervention 
group and 3.8 in the control group, $\mathrm{RR}=1.28, \mathrm{P}=0.006$; blood pressure self monitoring 3.6 versus 1.1 days per week, $R R=3.20$, $\mathrm{P}<0.001)$. The pooled results from the other four studies $(520$ participants; Analysis 6.1) gave an effect estimate of SMD 0.35, 95\% CI 0.17 to 0.52 , but with substantial heterogeneity between the studies. The effect reduced to $0.25,95 \% \mathrm{CI} 0.05$ to 0.44 and greater uncertainty with sensitivity analysis.

\section{Attainment of personal goals}

Only four of the 19 studies included a report on whether patients felt they had achieved the goals they had set for themselves, and all four gave positive results. Battersby 2007 reported a $60 \%$ improvement in problem and goals measurement scores; Glasgow 2005a found improvements in achievement of goals related to healthy eating and physical activity; Hart 1978 found a two-fold improvement in goal attainment among the intervention group which was better than that achieved by the control group; and Schillinger 2009 reported that $88 \%$ of participants in the intervention group had succeeded in developing their own goals and repeat action plans, leading to partial or complete success in goal achievement for an average of 2.5 plans per participant. The remaining 15 studies did not report on goal attainment.

\section{Health service use and costs}

Three studies included an estimate of the impact of personalised care planning on subsequent resource use (Table 9), but it was not possible to produce a pooled summary of these data.

The analysis reported in Battersby 2007 includes data from eight sub-studies, four of which were eligible for inclusion in our review. They concluded that the small observed reduction in hospital admissions was insufficient to pay for the costs of their model of coordinated care. These included costs associated with employing care co-ordinators, administering the trial, training care and service co-ordinators, and engaging service providers.

Wilson 2010 found improvements in medication use associated with personalised care planning, but did not assess whether these were cost-effective.

Katon 2010a included a formal analysis of the cost effectiveness of personalised care from the perspective of the health system. They found that, over 24 months, intervention participants had a mean of 114 (95\% CI 79 to 149 ) additional depression-free days and an estimated 0.335 (95\% CI -0.18 to 0.85 ) additional qualityadjusted life years (QALYs). Intervention participants also had lower mean outpatient health costs of USD 594 per participant (95\% CI -3241 to 2053 ) relative to usual care participants. They concluded that the intervention (TEAMcare) delivered high value for no or modest additional cost.

\section{Subgroup analysis: Effect of type of intervention}

We found evidence in relation to $\mathrm{HbA} 1 \mathrm{c}$ of differences in effect due to the type of intervention used (Table 2 and Table 3). Extended interventions covering five or more stages in the care-planning cycle (MD $-0.43,95 \%$ CI -0.60 to $-0.26 ; 3$ trials, 408 participants) were more effective than those that were limited to four or fewer (MD -0.12, 95\% CI -0.26 to 0.02 ; 6 trials, 1508 participants) (Analysis 7.1). High-intensity interventions (those involving one or more contacts a month for more than three months) (MD $0.43,95 \%$ CI -0.63 to $-0.24 ; 5$ trials, 847 participants) were more effective than low-intensity ones (MD - $0.17,95 \%$ CI -0.29 to 0.04; 4 trials, 1069 participants) (Analysis 8.1) and integrated interventions (those where the patient's usual clinician was informed about the patients' goals and action plans) (MD - $0.45,95 \% \mathrm{CI}$ -0.70 to $-0.21 ; 2$ trials, 358 participants) resulted in greater improvement than those that were not integrated (MD - 0.19, 95\% CI -0.31 to -0.08; 7 trials, 1558 participants) (Analysis 9.1).

We were not able to repeat these comparisons for the other outcome measures due to the small number of studies in each group. Nor were we able to examine the effect of the clinician's role, for example differences between those interventions that focused on changing clinicians' behaviour as well as that of patients, or those that involved contact with specially trained clinicians or peers in addition to the patient's usual-care clinicians versus those that relied on usual-care clinicians only.

\section{Subgroup analysis: Effect of type of participant}

Only one study included a formal measure of health literacy (Schillinger 2009), so we were unable to produce a pooled assessment of its effect on outcomes. No studies focused exclusively on patients with multi-morbidities, so our original intention to assess the effects of personalised care planning for these patients remains unfulfilled.

Of the five studies that recruited a majority of participants from lower socio-economic groups or minority ethnic populations, three found improvements on some outcome measures.

\section{DISCUSSION}

Despite the proliferation of studies of various aspects of long-term condition management, personalised care planning (as we defined it) has been assessed in a relatively small number of randomised controlled trials. We found 19 trials that fitted our definition; in other words, they had evaluated interventions designed to encourage and support patients to play an active role in identifying their own goals, determining priorities, and developing plans collaboratively with clinicians.

Fifteen out of the 19 studies reported positive effects for at least one outcome measure. The four studies that found no difference in effect between intervention and control groups (Frosch 2011; 
Glasgow 2005a; Kennedy 2013; Ludman 2007) evaluated low-intensity interventions, and all but one (Kennedy 2013) were 'addons' with no direct involvement of the patient's usual-care clinicians. Ludman 2007 was a small pilot for the larger Katon 2010a study and as such was not powered to distinguish effects between the groups. Kennedy 2013 had problems due to poor fidelity to the intervention, which may explain the lack of effect.

We found moderate-quality evidence that personalised care planning leads to improvements in physical health (blood glucose levels), psychological health (depression), self-management capabilities (self efficacy) and health behaviours (self-care activities). Evidence of impact on condition-specific health status, medication adherence, exercise frequency, resource use and cost effectiveness was mixed. We found no evidence of effects on diastolic blood pressure, cholesterol, body mass index, generic health status, or diet. Interventions that were more comprehensive, more intensive, and integrated into routine care achieved greater benefit than those that were limited, low intensity or not integrated.

Our review suggests that personalised care planning to identify patients' needs for clinical care and self-management support offers promise as an effective way of improving health outcomes for people with long-term conditions.

\section{Summary of main results}

\section{Physical health}

Eleven studies measured the effects of personalised care planning on various clinical indicators of physical health. Six out of nine studies found improvements in glycated haemoglobin for the intervention group as compared to the control group. Combining these in a meta-analysis gave moderate confidence that personalised care planning for people with diabetes was effective for improving blood glucose control. Six studies included blood pressure among the outcome measures, and the pooled results showed that personalised care planning contributed to a small reduction in systolic blood pressure, but not diastolic. A single study found improvements in lung function and control among asthma patients. No effects were observed on cholesterol levels or body mass index.

\section{Psychological health (depression)}

Three out of six studies that measured symptoms of depression reported improvements. The pooled results for five of these studies showed that personalised care planning led to a reduction in symptoms of depression. We were unable to include one study in the pooled analysis due to differences in the way outcome measures were reported. This study found greater improvement in the control group than the intervention group.

\section{Subjective health status (generic and condition-specific)}

Impact on subjective health status or quality of life was measured using a variety of different scales, making it difficult to produce a pooled estimate. Six studies measured the effects on generic (as opposed to condition-specific) health status, but only one reported a significant improvement related to personalised care planning. Three studies measured generic health status using the physical component score (PCS) and mental component score (MCS) of the SF-36 and SF-12. We pooled results from these and found no effect. Condition-specific measures of health status are often found to be more sensitive to small effects than generic measures. Four of the included studies measured condition-specific health status (three for diabetes and one for asthma): the pooled results showed a small improvement associated with personalised care planning.

\section{Self-management capabilities}

Personalised care planning appears to have a positive effect on people's confidence and skills to self-manage their long-term condition. Seven out of eleven studies that measured the effect on selfmanagement capabilities found improvements. Six studies used comparable instruments to measure self efficacy (one relevant indicator of self-management capabilities). After excluding one large pragmatic trial (Kennedy 2013) in which the intervention was not implemented by clinicians as intended (and was therefore at high risk of bias), pooled results from the remaining five studies showed a small effect favouring personalised care planning.

\section{Secondary outcomes}

Ten studies measured the effects of personalised care planning on various health-related behaviours, including exercise, diet, medication adherence and self-care activities such as blood glucose monitoring or foot care, nine of which found improvements in one or more of these measures. We found a positive effect on self-care activities associated with personalised care planning and a small positive but non-significant effect on daily exercise levels. We were unable to pool data for the other behaviours due to the variety of measures used.

Only three studies included an estimate of impact on resource use and only two of these included cost data. One study found that the intervention was cost-effective, whereas the other study concluded that any savings due to reduced hospitalisation rates were outweighed by the costs of the intervention.

\section{Subgroups}

We had hoped to be able to compare the effects of several different facets of interventions on the full range of outcomes. The comparisons we considered important were as follows: those interventions where most of the stages of the care planning cycle were completed (extended) compared to those that completed only four or fewer 
(limited); those that attempted to change both clinicians' and patients' behaviours against those focused on patients' behaviour only; those classified as high-intensity compared to those that were low; and those that were integrated into the patient's usual care compared to those that were provided as additional services. In the event we had too few studies to carry out these comparisons for any of the outcomes apart from blood glucose measurement, which is applicable to patients with diabetes only. In this case we found that the effects of personalised care planning were greater when more stages of the care planning cycle were completed, when contacts between patients and health professionals were more frequent, and when the patient's usual clinician was involved in the process.

We were unable to estimate the relative effects of personalised care planning on participants with low as opposed to high health literacy, or on those with multiple long-term conditions compared to those with one condition only, because we found only one study that included a formal measurement of health literacy and none of the included studies focused explicitly on multi-morbidity. The results from the five studies that included a majority of participants with lower socio-economic status or from minority ethnic groups were mixed.

\section{Overall completeness and applicability of evidence}

The trials included in our review evaluated complex interventions applied mainly, but not exclusively, in primary care settings and involving various different patient groups with different medical diagnoses and different cultural, ethnic and socio-economic backgrounds. Thirteen of the 19 studies were conducted in the USA and 12 of the 19 focused on diabetes. We do not know if the results are generalisable to other settings and other patient groups, but we have no reason to think they are not. There were differences between the included studies in their stated aims and theoretical underpinnings. Few of the authors described personalised care planning in precisely the same manner that we have adopted for this review, and it was not always the primary focus of the evaluations. While all the studies included personalised care planning as a major component, they involved a variety of additional selfmanagement tools and techniques, including information packages, worksheets, group visits, educational interventions, and peer coaching. We cannot therefore assume that the observed effects were due solely to the planning process itself.

The interventions were delivered by a range of different types of clinicians, including doctors, nurses, other therapists and in two cases, patients as peer coaches (Thom 2013; Van der Wulp 2012). In some cases the work of these clinicians or coaches was carefully structured (including scripts or prompts to guide discussions with patients) and tightly supervised, while other studies relied on brief training courses only. Other interventions were more pragmatic in that they involved usual-care clinicians in the delivery the inter- vention. This was harder to control and two of the larger studies (Battersby 2007; Kennedy 2013) had problems persuading clinicians to adhere to study protocols, weakening any effects of the intervention.

The wide diversity of outcome measures used in these trials hampered our efforts to pool the data to some extent. Also, the diversity of outcome measures did not necessarily reflect full coverage of important outcome domains. This was especially true in relation to self-management capabilities. We were able to pool results from studies that measured the effect of the intervention on self efficacy, but self efficacy refers to self-perceived cognitive abilities, and the standard measures of self efficacy tend to focus on ability to carry out medically defined tasks. Self-management capability is a broader concept that refers to the capacity and opportunity to manage a condition, to attain valued goals, and more generally live well with the condition. Research to date has not adequately addressed the effects of personalised care planning on people's socially shaped and observed opportunities to manage and live well with long-term conditions.

We were unable to assess the likely impact of personalised care planning on people with multiple long-term conditions or on those with low health literacy. Four of the included studies focused on populations with low socio-economic status, but the diversity of approaches and outcome measures made it impossible to make a reliable assessment of the extent to which the effects may vary between these population subgroups.

\section{Quality of the evidence}

Risk of bias was an issue for many of the included studies. None of the studies was assessed as having a high risk of bias in relation to random sequence generation or allocation concealment, but 11 studies provided unclear or no information on these issues. Blinding of participants is almost impossible with this type of intervention and none of the studies achieved this, so we focused on blinding of outcome assessment, which was adequate in nine studies, unclear in nine studies, and high risk in one study. Detection bias, attrition bias and reporting bias should be less problematic, but several studies failed to report these risks adequately and three were assessed as having a high risk of bias in respect of these factors.

We pooled results from studies with different outcome measures that appeared to be measuring the same or very similar constructs (e.g. depression symptoms, self efficacy), but this will have introduced a degree of heterogeneity. We ignored outcome measures that did not appear to have been validated, but we did not attempt an independent assessment of the psychometric properties of the included measures. In certain cases (e.g. self-management capabilities) we had concerns about the measures used: in many cases these were medically focused and might not have tapped into the factors that were most important to individual patients. We excluded studies where people's choices were restricted to a predetermined set of very narrowly defined goals, but in most cases 
there were limits to the options considered, undermining the extent to which the process could be said to be truly personalised. It is interesting, and worrying, to note how few studies (only four) included reports on the extent to which patients achieved their personal goals.

Several of the studies were too small and underpowered to detect an effect. Some suffered high rates of attrition and the more naturalistic (pragmatic) studies had problems in encouraging clinicians to implement the intervention as designed. This was a particular problem for the largest study in the group (Kennedy 2013), so we excluded this one from the pooled analysis of effects on self efficacy (the only outcome measure in this study that was possible to pool) because its negative findings would have swamped the others, giving a distorted result.

\section{Potential biases in the review process}

Identifying relevant studies in this broad topic area was challenging. We searched a wide variety of databases, including trial registers and lists of unpublished sources such as $\mathrm{PhD}$ theses. We scanned reference lists for relevant studies and we searched for additional papers reporting other aspects of eligible studies, such as protocols and additional findings.

Two review authors, working independently, carried out study identification and data extraction, and referred any disagreements to a third review author for resolution. Although we were very careful not to discard relevant studies, we cannot discount the possibility that we may have missed some. In certain cases the interventions were poorly described, making it difficult to judge whether or not personalised care planning had taken place. In cases of doubt, we excluded the studies. The trials listed in the Characteristics of excluded studies table are those that we actively considered for possible inclusion but eventually discarded after discussion by three of the review authors. Most of the excluded studies described interventions that in our view were not truly collaborative.

We used fixed-effect meta-analysis because of the small number of studies in each analysis. This carries the risk that it may yield confidence limits that are too narrow. We checked this by doing a sensitivity analysis using a random-effects model, and found it made no difference to the main findings, apart from self efficacy where the positive effect would disappear.

\section{Agreements and disagreements with other studies or reviews}

A 2009 overview of systematic reviews concluded that while there was good evidence that the processes involved in personalised care planning would engage patients more effectively in managing their care, there was little evidence for an impact on health outcomes of doing so (Graffy 2009). We have now shown that there are indeed health benefits from this approach. Other reviews have examined various tools or interventions designed to inform and engage patients, such as decision aids (Stacey 2014), contracts (Bosch-Capblanch 2007), training for health professionals (Dwamena 2012), interactive health communication applications (Murray 2005), and a variety of methods to promote shared decision making (Legare 2014). For the most part these reviews found evidence of beneficial effects on the process of care but not on the outcomes. They focused on specific interventions designed to promote more collaborative forms of decision making and looked at whether the interventions produced the desired effect (i.e. shared decision making). Our starting point was different. We selected studies where a collaborative approach (personalised care planning) had been adopted as the intervention, and we assessed the effects of this on patient outcomes. The distinction is important because it led to the inclusion of a different set of studies, and hence a different assessment of the effects of this type of collaboration between patients and clinicians.

Evidence is accumulating that group-based self-management education can lead to improvements in some health outcome measures (Brady 2013; Foster 2007; Franek 2013; Steinsbekk 2012), but attending a weekly course does not suit everyone and problems with availability, infrequent referral by GPs, and low rates of uptake by patients have been reported, particularly amongst those groups most in need of self-management support (Jordan 2007). One-to-one personalised care planning, coupled with appropriate information, health coaching, problem-solving support and care co-ordination may be a better solution for these people, especially if it is relatively intensive and integrated into routine care (Williams 2011a). Implementing all elements of the Chronic Care Model (patient self-management support, use of clinical information systems, delivery system redesign, provider decision support, linkage to community resources, and organisational development) could help to ensure that the outcome improvements are sustained (Woltmann 2012).

\section{A U THORS' CONCLUSIONS}

\section{Implications for practice}

Personalised care planning leads to improvements in certain indicators of physical and psychological health status and people's ability to self-manage their condition, when compared to usual care. The effects appear to be greater when the intervention is more comprehensive, intensive and well-integrated into routine care. Evidence on the relative cost effectiveness of this approach is limited and uncertain.

In its ideal form, personalised care planning is fundamentally different from usual care (Burt 2012). It involves shared control of the consultation and a focus on the patient as a whole person, not just 
their specific condition. It includes support for self management and behaviour change, and should be the means by which care is co-ordinated and integrated around the individual. Achieving this in practice would require fundamental changes to the organisation and delivery of primary care in most countries to enable a more proactive, anticipatory and integrated approach (Coulter 2013; Stellefson 2013). The context of primary care differs from country to country, so any intervention to support new models of care must be carefully tailored to local circumstances. It will probably require training for health professionals in how to elicit patients' goals and priorities, while avoiding the imposition of an overly directive model of care that could undermine patients' confidence to self-manage their conditions (Williams 2011b). Shared decision making and non-directive motivational interviewing are well-described methods or competencies that might provide the core of these training schemes (Elwyn 2014), but their introduction into routine care needs to be tailored to local circumstances.

The evidence gathered here suggests that investment in relevant training, support and system redesign could lead to better outcomes for people with long-term conditions. We found some evidence that more intensive and better integrated approaches to personalised care planning and self-management support may work best, but heterogeneity and uncertainties among both the interventions and the outcome measures mean that current evidence cannot support a specific blueprint for widespread adoption. Nevertheless, our review offers a comprehensive conceptual model that we hope will inform future interventions.

\section{Implications for research}

We found positive effects on blood glucose, blood pressure, depression, condition-specific health status, self-management capabilities, and self-care activities. This is encouraging, but more trials are required to check the robustness of these findings in diverse settings and to determine which elements of these complex interventions are most likely to be effective.

Support for self management of long-term conditions is a growing area of research. We identified published protocols for seven ongoing studies that may help to address important uncertainties about the effects of personalised care planning. Future studies should examine its impact on patients' self-management capabilities, health behaviours, goal attainment, and resource use, in addition to clinical indicators and psychological outcomes. Studies should focus on longer-term outcomes and include measures of resource use and costs. More studies are also needed to compare outcomes for patients at different levels of health literacy. The lack of studies on multi-morbidity is a serious gap in knowledge that has been noted before (Barnett 2012). Since personalised care planning is potentially an effective way to co-ordinate care for people with multiple health problems, we would hope to see many more studies addressing this issue.
Ideally trials should be conducted in real-life settings, but this is a difficult topic to study in cluster trials because it involves complex behaviour change and a time commitment from those delivering the intervention. Ensuring fidelity to the protocol can be very difficult in these situations, as evidenced by the experience of the investigators involved in Kennedy 2013. Their very useful process review (Kennedy 2014) should be read by all researchers planning future trials in this field.

Greater standardisation of outcome measures would be very helpful for future systematic reviews and meta-analyses. Subjective health status and self-management capabilities are key outcomes in studies of care and support for people with chronic conditions, but the field will not advance until there is greater agreement on how to measure these constructs. It is of some concern that so few published studies in this field have attempted to find out whether patients attained their personal goals, as opposed to those determined by clinicians or researchers. This implies a disrespect for patients' interests, values and capabilities-the antithesis of personalised care. Most studies in our review adopted a limited view of patients' capabilities, usually restricted to managing health-related procedures and behaviour change. This ignores many of the other resources that individuals, families and communities can contribute to health improvement. We also need more in-depth information about patients' experience of personalised care planning to determine which models work best, for whom and in what circumstances. We fervently hope that future studies will remedy these gaps, perhaps by including patient-generated outcome measures, by conducting qualitative research alongside the randomised trials, or both.

\section{ACKNOWLEDGEMENTS}

We received very helpful advice from the following members of the expert patient advisory group: Carole Bennett, Graham Brown, Eleni Chambers, Lesley Cox, Linda Eccles, and Margo Milne. Their input was invaluable. We also received significant help from Adrian Edwards, Sophie Hill, Bronwyn Hemsley, Joanne Protheroe and Anne Lyddiatt, who reviewed the draft protocol. We are most grateful to Nia Roberts of the Bodleian Health Care Libraries, University of Oxford, and John Kis-Rigo of the Cochrane Consumers and Communication Review Group for help in designing the search strategy. Several study authors kindly responded to our requests for further information: for this we owe particular thanks to Malcolm Battersby, Evette Ludman, Aanand Naik, Victoria Stanhope and Sandra Wilson.

Angela Coulter and Sara Ryan are members of the Policy Research Unit on Quality and Outcomes of Person-centred Care (QORU) (www.qoru.ac.uk), This is an independent report commissioned from QORU by the Policy Research Programme in the Depart- 
ment of Health in England. The views expressed are not necessar-

ily those of the Department.

\section{R E F E R E N C E S}

\section{References to studies included in this review}

\section{Battersby 2007 \{published data only (unpublished sought but not used)\}}

* Battersby M, Harvey P, Mills PD, Kalucy E, Pols RG, Frith

PA, et al. SA HealthPlus: a controlled trial of a statewide

application of a generic model of chronic illness care. The

Millbank Quarterly 2007;85(1):37-67.

Battersby MW. Health reform through coordinated care:

SA HealthPlus. BMJ 2005;330(7492):662-6.

Battersby MW, Ask A, Reece MM, Markwick MJ, Collins

JP. A case study using the "Problems and Goals Approach" in a coordinated care trial: SA HealthPlus. Australian Journal of Primary Health 2001;7(3):45-8.

Battersby MW, Ask A, Reece MM, Markwick MJ, Collins JP. The Partners in Health scale: the development and psychometric properties of a generic assessment scale for chronic condition self-management. Australian Journal of Public Health 2003;9(2/3):41-52.

Heard A, Kalucy E, Richardson D, Battersby MW, Frith P, McGowan C. An evaluation of the care planning process in coordinated care trials: what difference do care plans make to service provision?. Australian Journal of Primary Health 2002;8(1):52-6.

Pols RG, Battersby MW. Coordinated care in the management of patients with unexplained physical symptoms: depression is a key issue. Medical Journal of Australia 2008;188(12 Suppl):S133-7.

Frosch 2011 \{published data only\}

Frosch DL, Uy V, Ochoa S, Mangione CM. Evaluation of a behavior support intervention for patients with poorly controlled diabetes. Archives of Internal Medicine 2011; Vol. 171, issue 22:2011-7.

Glasgow 2005a \{published data only (unpublished sought but not used)\}

Glasgow RE, Nutting PA, King DK, Nelson CC, Cutter G, Gaglio B, et al. A practical randomized trial to improve diabetes care. Journal of General Internal Medicine 2004a;19 (12):1167-74.

* Glasgow RE, Nutting PA, King DK, Nelson CC, Cutter

G, Gaglio B, et al. Randomized effectiveness trial of a computer-assisted intervention to improve diabetes care. Diabetes Care 2005a;28(1):33-9.

Hart 1978 \{published data only\}

Hart RR. Therapeutic effectiveness of setting and monitoring goals. Journal of Consulting and Clinical Psychology 1978;46(6):1242-5.

Hiss 2007 \{published data only\}

Hiss RG, Armbruster BA, Gillard ML, McClure LA. Nurse care manager collaboration with community-based physicians providing diabetes care: a randomized controlled trial. Diabetes Educ 2007;33(3):493-502.

\section{Katon 2010a \{published data only\}}

Katon W, Lin EH, Von Korff M, Ciechanowski P, Ludman $\mathrm{E}$, Young B, et al. Integrating depression and chronic disease care among patients with diabetes and/or coronary heart disease: the design of the TEAMcare study. Contemporary Clinical Trials 2010b;31(4):312-22.

Katon W, Russo J, Lin EH, Schmittdiel J, Ciechanowski P, Ludman E, et al. Cost-effectiveness of a multicondition collaborative care intervention: a randomized controlled trial. Archives of General Psychiatry 2012;69(5):506-14.

* Katon WJ, Lin EH, Von Korff M, Ciechanowski P, Ludman EJ, Young B, et al. Collaborative care for patients with depression and chronic illnesses. New England Journal of Medicine 2010a;363(27):2611-20.

Lin EH, Von Korff M, Ciechanowski P, Peterson D, Ludman EJ, Rutter CM, et al. Treatment adjustment and medication adherence for complex patients with diabetes, heart disease, and depression: a randomized controlled trial. Annals of Family Medicine 2012;10(1):6-14.

Ludman EJ, Peterson D, Katon WJ, Lin EH, Von Korff M, Ciechanowski P, et al. Improving confidence for self care in patients with depression and chronic illnesses. Behavioral Medicine 2013;39(1):1-6.

Von Korff M, Katon WJ, Lin EH, Ciechanowski P, Peterson D, Ludman EJ, et al. Functional outcomes of multicondition collaborative care and successful ageing: results of randomised trial. BMJ 2011;343:d6612.

\section{Kennedy 2013 \{published data only\}}

Bower P, Kennedy A, Reeves D, Rogers A, Blakeman T, Chew-Graham C, et al. A cluster randomised controlled trial of the clinical and cost-effectiveness of a 'whole systems' model of self-management support for the management of long- term conditions in primary care: trial protocol. Implementation Science 2012;7:7.

Fullwood C, Kennedy A, Rogers A, Eden M, Gardner C, Protheroe J, et al. Patients' experiences of shared decision making in primary care practices in the United kingdom. Medical Decision Making 2013;33(1):26-36.

* Kennedy A, Bower P, Reeves D, Blakeman T, Bowen R, Chew-Graham C, et al. Implementation of self management support for long term conditions in routine primary care settings: Cluster randomised controlled trial. BMJ (Online) 2013;346:f2882. [DOI: 10.1136/bmj.f2882] Kennedy A, Chew-Graham C, Blakeman T, Bowen A, Gardner C, Protheroe J, et al. Delivering the WISE (Whole Systems Informing Self-Management Engagement) training package in primary care: learning from formative 
evaluation. Implementation Science 2010;29(5):7.

Kennedy A, Rogers A, Chew-Graham C, Blakeman T, Bowen R, Gardner C, et al. Implementation of a selfmanagement support approach (WISE) across a health system: a process evaluation explaining what did and did not work for organisations, clinicians and patients. Implementation Science 2014;9:129.

Protheroe J, Blakeman T, Bower P, Chew-Graham C, Kennedy A. An intervention to promote patient participation and self-management in long term conditions: development and feasibility testing. BMC Health Services Research 2010;10:206.

Liu 2012 \{published data only\}

Liu S, Bi A, Fu D, Fu H, Luo W, Ma X, et al. Effectiveness of using group visit model to support diabetes patient self-management in rural communities of Shanghai: a randomized controlled trial. BMC Public Health 2012;12: 1043.

Ludman 2007 \{published and unpublished data\} Ludman EJ, Simon GE, Grothaus LC, Luce C, Markley DK, Schaefer J. A pilot study of telephone care management and structured disease self-management groups for chronic depression. Psychiatric Services 2007;58(8):1065-72.

Naik 2011 \{published data only\}

Naik AD, Palmer N, Petersen NJ, Street RL Jr, Rao R, Suarez-Almazor M, et al. Comparative effectiveness of goal setting in diabetes mellitus group clinics: randomized clinical trial. Archives of Internal Medicine 2011;171(5): 453-9.

Schillinger 2009 \{published data only\}

Handley M, MacGregor K, Schillinger D, Sharifi C, Wong $S$, Bodenheimer T. Using action plans to help primary care patients adopt healthy behaviors: a descriptive study. Journal of the American Board of Family Medicine 2006;19 (3):224-31.

Handley MA, Hammer H, Schillinger D. Navigating the terrain between research and practice: a collaborative research network (CRN) case study in diabetes research. Journal of the American Board of Family Medicine 2006;19 (1):85-92.

Handley MA, Shumway M, Schillinger D. Costeffectiveness of automated telephone self-management support with nurse care management among patients with diabetes. Annals of Family Medicine 2008;6(6):512-8. Schillinger D, Hammer H, Wang F, Palacios J, McLean I, Tang A, et al. Seeing in 3-D: examining the reach of diabetes self-management support strategies in a public health care system. Health Education \& Behavior 2008;35(5):664-82. * Schillinger D, Handley M, Wang F, Hammer H. Effects of self-management support on structure, process, and outcomes among vulnerable patients with diabetes: a threearm practical clinical trial. Diabetes Care 2009;32(4): 559-66.

Shearer 2007 \{published data only\} Shearer NB, Cisar N, Greenberg EA. A telephone-delivered empowerment intervention with patients diagnosed with heart failure. Heart \& Lung 2007;36(3):159-69.
Stanhope 2013 \{published and unpublished data\}

Stanhope V, Ingoglia C, Schmelter B, Marcus SC. Impact of person-centered planning and collaborative documentation on treatment adherence. Psychiatric Services 2013;64(1): 76-9.

Thom 2013 \{published data only\}

Ghorob A, Vivas MM, De Vore D, Ngo V, Bodenheimer

$\mathrm{T}$, Chen $\mathrm{E}$, et al. The effectiveness of peer health coaching in improving glycemic control among low-income patients with diabetes: protocol for a randomized controlled trial. BMC Public Health 2011;11:208.

* Thom DH, Ghorob A, Hessler D, De Vore D, Chen $\mathrm{E}$, Bodenheimer TA. Impact of peer health coaching on glycemic control in low-income patients with diabetes: a randomized controlled trial. Annals of Family Medicine 2013;11(2):137-44.

Tsay 2004 \{published data only\}

Tsay SL, Hung LO. Empowerment of patients with end-stage renal disease-a randomized controlled trial. International Journal of Nursing Studies 2004;41(1):59-65.

Van der Wulp 2012 \{published data only\}

Van der Wulp I, De Leeuw JR, Gorter KJ, Rutten GE. Effectiveness of peer-led self-management coaching for patients recently diagnosed with Type 2 diabetes mellitus in primary care: a randomized controlled trial. Diabetic Medicine 2012;29(10):e390-7.

Wilson 2010 \{published and unpublished data\} Wilson SR, Strub P, Buist AS, Knowles SB, Lavori PW, Lapidus J, et al. Shared treatment decision making improves adherence and outcomes in poorly controlled asthma. American Journal of Respiratory and Critical Care Medicine 2010;181(6):566-77.

Wolever 2010 \{published data only\} Wolever RQ, Dreusicke M, Fikkan J, Hawkins TV, Yeung $S$, Wakefield J, et al. Integrative health coaching for patients with type 2 diabetes: a randomized clinical trial. Diabetes Educator 2010;36(4):629-39.

Zoffmann 2006 \{published data only (unpublished sought but not used)\}

Zoffmann V, Lauritzen T. Guided self-determination improves life skills with type 1 diabetes and $\mathrm{A} 1 \mathrm{C}$ in randomized controlled trial. Patient Education and Counseling 2006;64(1-3):78-86.

\section{References to studies excluded from this review}

Alamo 2002 \{published data only\}

Alamo MM, Moral RR, Pérula de Torres LA. Evaluation of a patient-centred approach in generalized musculoskeletal chronic pain/fibromyalgia patients in primary care. Patient Education and Counseling 2002;48(1):23-31.

Anderson 2005 \{published data only\} Anderson RM, Funnell MM, Nwanko R, Gillard ML, Oh M, Fitzgerald JT. Evaluating a problem-based empowerment program for African Americans with diabetes: results of a randomized controlled trial. Ethnicity \& Disease 2005; 15 (4):671-8. 
Bieber 2006 \{published data only\}

Bieber C, Müller KG, Blumenstiel K, Hochlehnert A, Wilke $S$, Hartmann M, et al. A shared decision-making communication training program for physicians treating fibromyalgia patients: Effects of a randomized controlled trial. Journal of Psychosomatic Research 2008;64(1):13-20. * Bieber C, Müller KG, Blumenstiel K, Schneider A, Richter A, Wilke $S$, et al. Long-term effects of a shared decisionmaking intervention on physician-patient interaction and outcome in fibromyalgia: a qualitative and quantitative 1 year follow-up of a randomized controlled trial. Patient Education and Counseling 2006;63(3):357-66.

Brown 2005 \{published data only\}

Brown SA, Blozis SA, Kouzekanani K, Garcia AA, Winchell M, Hanis CL. Dosage effects of diabetes self-management education for Mexican Americans: the Starr County Border Health Initiative. Diabetes Care 2005;28(3):527-32.

Chambers 2008 \{published data only\}

Chambers SK, Ferguson M, Gardiner RA, Nicol D, Gordon L, Occhipinti $S$, et al. ProsCan for men: randomised controlled trial of a decision support intervention for men with localised prostate cancer. BMC Cancer 2008;8:207.

Chin 2007 \{published data only\}

Chin MH, Drum ML, Guillen M, Rimington A, Levie JR, Kirchhoff AC, et al. Improving and sustaining diabetes care in community health centers with the health disparities collaboratives. Medical Care 2007;45(12):1135-43.

Coleman 2006 \{published data only\} Coleman EA, Parry C, Chalmers S, Min SJ. The Care Transitions intervention: results of a randomized controlled trial. Archives of Internal Medicine 2006;166(17):1822-8.

Cooper 2011 \{published data only\}

Cooper LA, Roter DL, Carson KA, Bone LR, Larson SM, Miller ER, et al. A randomised trial to improve patientcentered care and hypertension control in underserved primary care patients. Journal of General Internal Medicine 2011;26(11):1297-304.

Cooper 2013 \{published data only\} Cooper LA, Ghods Dinoso BK, Ford DE, Roter DL, Primm $A B$, Larson SM, et al. Comparative effectiveness of standard versus patient-centered collaborative care interventions for depression among African Americans in primary care settings: the BRIDGE Study. Health Services Research 2013; 48(1): 150-74.

Deen 2011 \{published data only\}

Deen TL, Fortney JC, Pyne JM. Relationship between satisfaction, patient-centered care, adherence and outcomes among patients in a collaborative care trial for depression. Administration and Policy in Mental Health 2011;38(5): 345-55.

Druss 2010 \{published data only\}

Druss BG, Zhao L, Von Esenwein SA, Bona JR, Fricks L, Jenkins-Tucker $S$, et al. The Health and Recovery Peer (HARP) Program: a peer-led intervention to improve medical self-management for persons with serious mental illness. Schizophrenia Research 2010;118(1-3):264-70.
Eakin 2007 \{published data only\}

Eakin EG, Bull SS, Riley KM, Reeves MM, McLaughlin P, Gutierrez $S$. Resources for health: a primary-care-based diet and physical activity intervention targeting urban latinos with multiple chronic conditions. Health Psychology 2007; 26(4):392-400.

Estabrooks 2005 \{published data only\} Estabrooks PA, Nelson CC, Xu S, King D, Bayliss EA, Gaglio B, et al. The frequency and behavioral outcomes of goal choices in the self-management of diabetes. The Diabetes Educator 2005;31(3):391-400.

Glasgow 2010 \{published data only\} Glasgow RE, Kurz D, King D, Dickman JM, Faber AJ, Halterman E, et al. Outcomes of minimal and moderate support versions of an internet-based diabetes selfmanagement support program. Journal of General Internal Medicine 2010;25(12):1315-22.

Halpern 2004 \{published data only\} Halpern J, Johnson M D, Miranda J, Wells K B. The partners in care approach to ethics outcomes in quality improvement programs for depression. Psychiatric services 2004;55(5):532-9.

Hamann 2006 \{published data only\} Hamann J, Langer B, Winkler V, Busch R, Cohen R, Leucht $S$, et al. Shared decision making for in-patients with schizophrenia. Acta Psychiatrica Scandinavica 2006;114(4): 265-73.

Harris 2009 \{published data only\}

Harris N, Lovell K, Day J, Roberts C. An evaluation of a medication management training programme for community mental health professionals; service user level outcomes: a cluster randomised controlled trial. International Journal of Nursing Studies 2009;46(5):645-52.

\section{Heisler 2013 \{published data only\}}

Heisler M, Halasyamani L, Cowen ME, Davis MD, Resnicow K, Strawderman RL, et al. Randomized controlled effectiveness trial of reciprocal peer support in heart failure. Circulation. Heart Failure 2013;6(2):246-53.

\section{Joosten 2011 \{published data only\}} Joosten EA, De Jong CA, De Weert-van Oene GH, Sensky T, Van der Staak CP. Shared decision-making: increases autonomy in substance-dependent patients. Substance Use \& Misuse 2011;46(8):1037-8.

Kilbourne 2013 \{published data only\} Kilbourne AM, Li D, Lai Z, Waxmonsky J, Ketter T. Pilot randomized trial of a cross-diagnosis collaborative care program for patients with mood disorders. Depression and Anxiety 2013;30(2):116-22.

Koelewijn-van Loon 2010 \{published data only\} Koelewijn-van Loon MS, Van der Weijden T, Ronda G, Van Steenkiste B, Winkens B, Elwyn G, et al. Improving lifestyle and risk perception through patient involvement in nurse-led cardiovascular risk management: a clusterrandomized controlled trial in primary care. Preventive Medicine 2010;50(1-2):35-44. 
Lin 2006 \{published data only\}

Lin EH, Katon W, Rutter C, Simon GE, Ludman EJ, Von Korff M, et al. Effects of enhanced depression treatment on diabetes self-care. Annals of Family Medicine 2006;4(1): $46-53$.

Maindal 2011 \{published data only\}

Maindal HT, Sandbæk A, Kirkevold M, Lauritzen T. Effect on motivation, perceived competence, and activation after participation in the "Ready to Act" programme for people with screen-detected dysglycaemia: a 1-year randomised controlled trial, Addition-DK. Scandinavian Journal of Public Health 2011; Vol. 39, issue 3:262-71.

McKay 2002 \{published data only\}

McKay GH, Glasgow RE, Feil EG, Boles SM, Barrera M. Internet-based diabetes self-management and support: Initial outcomes from the diabetes network project. Rehabilitation Psychology 2002;47(1):31-48.

Patja 2012 \{published data only\}

Patja K, Absetz P, Auvinen A, Tokola K, Kytö J, Oksman E, et al. Health coaching by telephony to support self-care in chronic diseases: clinical outcomes from The TERVA randomized controlled trial. BMC Health Services Research 2012; Vol. 12:147.

Redfern 2010 \{published data only\}

Redfern J, Briffa T, Ellis E, Freedman SB. Choice of secondary prevention improves risk factors after acute coronary syndrome: 1-year follow-up of the CHOICE (Choice of Health Options In prevention of Cardiovascular Events) randomised controlled trial. Heart 2009; Vol. 95, issue 6:468-75.

Richardson 2010 \{published data only\} Richardson J, Letts L, Chan D, Stratford P, Hand C, Price $\mathrm{D}$, et al. Rehabilitation in a primary care setting for persons with chronic illness: a randomized controlled trial. Primary Health Care Research and Development 2010; Vol. 11: 382-95.

Riley 2001 \{published data only\}

Riley KM, Glasgow RE, Eakin EG. Resources for Health: A social-ecological intervention for supporting selfmanagement of chronic conditions. Journal of Health Psychology 2001;6(6):693-705.

Ruggiero 2010 \{published data only\}

Ruggiero L, Moadsiri A, Butler P, Oros SM, Berbaum ML, Whitman S, et al. Supporting diabetes self-care in underserved populations: a randomized pilot study using medical assistant coaches. The Diabetes Educator 2010;36 (1):127-31

Ruland 2003 \{published data only\} Ruland CM, White T, Stevens M, Fanciullo G, Khilani SM. Effects of a computerized system to support shared decision making in symptom management of cancer patients: preliminary results. Journal of the American Medical Informatics Association 2003;10(6):573-9.

Sciamanna 2011 \{published data only\}

Sciamanna CN, Thiboutot J. Effects of a patient activation intervention to overcome clinical inertia to control blood pressure. Journal of General Internal Medicine 2011; Vol. 26:S112.

Simon 2002 \{published data only\}

Simon GE, Ludman E, Unutzer J, Bauer MS. Design and implementation of a randomized trial evaluating systematic care for bipolar disorder. Bipolar Disorders 2002;4(4): 226-36.

Simon 2011 \{published data only\}

Simon GE, Ludman EJ, Goodale LC, Dykstra DM, Stone $\mathrm{E}$, Cutsogeorge $\mathrm{D}$, et al. An online recovery plan program: can peer coaching increase participation?. Psychiatric Services 2011;62(6):666-9.

Smeulders 2009 \{published data only\}

Smeulders ES, Van Haastregt JC, Janssen-Boyne JJ, Stoffers HE, Van Eijk JT, Kempen GI. Feasibility of a group-based self-management program among congestive heart failure patients. Heart \& Lung 2009;38(6):499-512.

Smith 2008 \{published data only\}

Smith SA, Shah ND, Bryant SC, Christianson TJ, Bjornsen SS, Giesler PD, et al. Chronic care model and shared care in diabetes: randomised trial of an electronic decision support system [Erratum appears in Mayo Clin Proc. 2008 Oct;83 (10):1189]. Mayo Clinic Proceedings 2008;83(7):747-57.

\section{Sobell 2000 \{published data only\}}

Sobell MB, Sobell LC, Leo GI. Does enhanced social support improve outcomes for problem drinkers in guided self-change treatment?. Journal of Behavior Therapy and Experimental Psychiatry 2000;31(1):41-54.

Sol 2008 \{published data only\}

Sol BG, Van der Graaf Y, Van der Bijl JJ, Goessens BM, Visseren FL. The role of self-efficacy in vascular risk factor management: A randomized controlled trial. Patient Education and Counseling 2008;71(2):191-7.

Street 2010 \{published data only\}

Street RL, Slee C, Kalauokalani DK, Dean DE, Tancredi DJ, Kravitz RL. Improving physician-patient communication about cancer pain with a tailored education-coaching intervention. Patient Education and Counseling 2010;80(1): $42-7$.

Stringer 2011 \{published data only\}

Stringer B, Van Meijel B, Koekkoek B, Kerkhof A, Beekman A. Collaborative Care for patients with severe borderline and NOS personality disorders: a comparative multiple case study on processes and outcomes. BMC Psychiatry 2011;11: 102.

Van GestelTimmermans 2012 \{published data only\} Van Gestel-Timmermans H, Brouwers EP, Van Assen MA, Van Nieuwenhuizen C. Effects of a peer-run course on recovery from serious mental illness: a randomised controlled trial. Psychiatric Services 2012;63(1):54-60.

Vestala 2013 \{published data only\}

Vestala H, Frisman GH. Can participation in documentation influence experiences of involvement in care decisionmaking?. Open Nursing Journal 2013;7:66-72. 
Von Korff 2003 \{published data only\}

Von Korff M, Katon W, Rutter C, Ludman E, Simon G, Lin E, et al. Effect on disability outcomes of a depression relapse prevention program. Psychosomatic Medicine 2003; 65(6):938-43.

Walker 2005 \{published data only\}

Walker MS, Podbilewicz-Schuller Y. Video preparation for breast cancer treatment planning: results of a randomized clinical trial. Psycho-Oncology 2005;14(5):408-20.

Wennberg 2010 \{published data only\} Wennberg DE, Marr A, Lang L, O'Malley S, Bennett G. A randomized trial of a telephone care-management strategy. New England Journal of Medicine 2010;363(13):1245-55.

Woltmann 2011 \{published data only\} Woltmann EM, Wilkniss SM, Teachout A, McHugo GJ, Drake RE. Trial of an electronic decision support system to facilitate shared decision making in community mental health. Psychiatric Services 2011;62(1):54-60.

Wright 2003 \{published data only\} Wright SP, Walsh H, Ingley KM, Muncaster SA, Gamble GD, Pearl A, et al. Uptake of self-management strategies in a heart failure management programme. European Journal of Heart Failure 2003;5(3):371-80.

\section{References to ongoing studies}

\section{Altiner 2012 \{published data only\}}

Altiner A, Schäfer I, Mellert C, Löffler C, Mortsiefer A, Ernst A, et al. Activating GENeral practitioners Dialogue with patients on their Agenda (MultiCare AGENDA) study protocol for a cluster randomized controlled trial. $B M C$ Family Practice 2012;13:118.

Bachman-Mettler 2011 \{published data only\} Bachmann-Mettler I, Steurer-Stey C, Senn O, Wang M, Bardheci K, Rosemann T. Case management in oncology rehabilitation (CAMON): The effect of case management on the quality of life in patients with cancer after one year of ambulant rehabilitation. A study protocol for a randomized controlled clinical trial in oncology rehabilitation. Trials 2011;12:103.

Battersby 2010 \{published data only\} Battersby MW, Harris M, Reed RI, Harvey PW, Woodman RJ, Frith P. A randomised trial of the Flinders Program to improve patient self-management competencies in a range of chronic conditions: study rationale and protocol. Australasian Medical Journal 2010;1(3):198-204.

Coventry 2012 \{published data only\}

Coventry PA, Lovell K, Dickens C, Bower P, Chew-Graham C, Cherrington A, et al. Collaborative interventions for Circulation and Depression (COINCIDE): study protocol for a cluster randomized controlled trial of collaborative care for depression in people with diabetes and/or coronary heart disease. Trials 2012;13:139.

Reed 2011 \{published data only\}

Reed RL, Battersby M, Osborne RH, Bond MJ, Howard SL, Roeger L. Protocol for a randomised controlled trial of chronic disease self-management support for older Australians with multiple chronic diseases. Contemporary Clinical Trials 2011;32(6):946-52.

Tylee 2012 \{published data only\}

Barley EA, Haddad M, Simmonds R, Fortune Z, Walters P, Murray J, et al. The UPBEAT depression and coronary heart disease programme: using the UK medical research council framework to design a nurse-led complex intervention for use in primary care. BMC Family Practice 2012;13:119.

* Tylee A, Haddad M, Barley E, Ashworth M, Brown J, Chambers J, et al. A pilot randomised controlled trial of personalised care for depressed patients with symptomatic coronary heart disease in South London general practices: the UPBEAT-UK RCT protocol and recruitment. BMC Psychiatry 2012;12:58.

Van der Voort 2011 \{published data only\} Van der Voort TY, Van Meijel B, Goossens PJ, Renes J, Beekman AT, Kupka RW. Collaborative care for patients with bipolar disorder: a randomised controlled trial. $B M C$ Psychiatry 2011;11:133.

\section{Additional references}

\section{Anderson 2000}

Anderson RM, Fitzgerald JT, Funnell MM, Marrero DG. The diabetes empowerment scale. Diabetes Care 2000;23 (6):739-43.

\section{Bandura 1977}

Bandura A. Self-efficacy: toward a unifying theory of behavioral change. Psychological Review 1977;84(2): 191-215.

\section{Barnett 2012}

Barnett K, Mercer SW, Norbury M, Watt G, Wyke S, Guthrie B. Epidemiology of multi-morbidity and implications for health care, research, and medical education: a cross-sectional study. Lancet 2012;380(9836): $37-43$.

\section{Battersby 2005}

Battersby MW. Health reform through coordinated care: SA HealthPlus. BMJ 2005;330(7492):662-6.

\section{Beck 1988}

Beck AT, Steer RA, Garbin MG. Psychometric properties of the Beck Depression Inventory: twenty-five years of evaluation. Clinical Psychology Review 1988;8(1):77-100.

Blakeman 2006

Blakeman T, Macdonald W, Bower P, Gately C, ChewGraham C. A qualitative study of GPs' attitudes to selfmanagement of chronic disease. British Journal of General Practice 2006;56(527):407-14.

Bodenheimer 2003

Bodenheimer T. Interventions to improve chronic illness care: evaluating their effectiveness. Disease Management 2003;6(2):63-71.

Bosch-Capblanch 2007

Bosch-Capblanch X, Abba K, Prictor M, Garner P. Contracts between patients and healthcare practitioners 
for improving patients' adherence to treatment, prevention and health promotion activities. Cochrane Database of Systematic Reviews 2007, Issue 2. [DOI: 10.1002/ 14651858.CD004808.pub3]

\section{Bower 2012}

Bower P, Kennedy A, Reeves D, Rogers A, Blakeman T, Chew-Graham C, et al. A cluster randomised controlled trial of the clinical and cost-effectiveness of a 'whole systems' model of self-management support for the management of long- term conditions in primary care: trial protocol. Implementation Science 2012;7:7.

\section{Brady 2013}

Brady T J, Murphy L, O'Colmain B J, Beauchesne D, Daniels B, Greenberg M, et al. A meta-analysis of health status, health behaviors, and health care utilization outcomes of the chronic disease self-management program. Preventing Chronic Disease 2013;10:120112.

\section{Burt 2012}

Burt J, Roland M, Paddison C, Reeves D, Campbell J, Abel $G$, et al. Prevalence and benefits of care plans and care planning for people with long-term conditions in England. Journal of Health Services Research and Policy. 2012/02/15 2012; Vol. 17, issue Suppl 1:64-71.

Burt 2013

Burt J, Rick J, Blakeman T, Protheroe J, Roland M, Bower P. Care plans and care planning in long-term conditions: a conceptual model. Primary Health Care Research \& Development 2013;10:120112. [DOI: 10.1017/ S1463423613000327]

\section{Carey 1990}

Carey MP, Jorgensen RS, Weinstock RS, Sprafkin RP, Lantinga LJ, Carnrike CL, et al. Reliability and validity of the Appraisal of Diabetes scale. Journal of Behavioral Medicine 1991;14(1):43-51.

\section{Caroselli 1998}

Caroselli C, Barrett EA. A review of the Power as Knowing Participation in Change literature. Nursing Science Quarterly 1998;11(1):9-16.

\section{Charles 1999}

Charles C, Gafni A, Whelan T. Decision-making in the physician-patient encounter: revisiting the shared treatment decision-making model. Social Science and Medicine 1999; 49(5):651-61.

\section{Cohen 1983a}

Cohen S, Kamarck T, Mermelstein R. A global measure of perceived stress. Journal of Health and Social Behavior 1983; 24(4):385-96.

\section{Cohen 1983b}

Cohen S, Hobermnan H. Positive events and social supports as buffers of life change stress. Journal of Applied Social Psychology 1983;13:99-125.

\section{Coulter 2011}

Coulter A, Collins A. Making Shared Decision Making a Reality. London: King's Fund, 2011.

\section{Coulter 2013}

Coulter A, Roberts S, Dixon A. Delivering Better Services for People with Long-term Conditions: Building the House of Care. London: King's Fund, 2013.

\section{Deakin 2005}

Deakin TA, McShane CE, Cade JE, Williams R. Group based training for self-management strategies in people with type 2 diabetes mellitus. Cochrane Database of Systematic Reviews 2005, Issue 2. [DOI: 10.1002/ 14651858.CD003417.pub2]

\section{Department of Health 2009}

Department of Health/Long Term Conditions. Supporting people with long term conditions: commissioning personalised care planning. www.londonhp.nhs.uk/wpcontent/uploads/2011/03/EOLC-Supporting-People-withLTC.pdf 2009 (accessed 16 January 2015).

\section{Department of Health 2011}

Department of Health. Personalised care planning: improving care for people with long term conditions (Information Sheet 1). www.dh.gov.uk/prod consum d dh/ groups/dh digitalassets/documents/digitalasset/ dh 124048.pdf 2011 (accessed 21 Dec 2012).

\section{Department of Health 2012}

Department of Health. The Mandate. A Mandate from the Government to the NHS Commissioning Board: April 2013 to March 2015. www.cypiapt.org/site-files/1315 mandate.pdf 2012 (accessed 16 January 2015).

\section{Derogatis 1974}

Derogatis L, Rickels K, Uhlenhuth E, Covi L. The Hopkins Symptom Checklist: A Measure of Primary Symptom Dimensions. Basel, Switzerland: Kargerman, 1974.

\section{Devins 2010}

Devins GM. Using the illness intrusiveness ratings scale to understand health-related quality of life in chronic disease. Journal of Psychosomatic Research 2010;68(6):591-602.

\section{Dijkstra 2005}

Dijkstra RF, Braspenning JC, Huijsmans Z, Akkermans RP, Van Ballegooie E, Ten Have P, et al. Introduction of diabetes passports involving both patients and professionals to improve hospital outpatient diabetes care. Diabetes Research and Clinical Practice 2005;68(2):126-34.

\section{Dwamena 2012}

Dwamena F, Holmes-Rovner M, Gaulden CM, Jorgenson S, Sadigh G, Sikorskii A, et al. Interventions for providers to promote a patient-centred approach in clinical consultations. Cochrane Database of Systematic Reviews 2012, Issue 12. [DOI: 10.1002/14651858.CD003267.pub2]

\section{Edwards 2013}

Edwards AGK, Naik G, Ahmed H, Elwyn GJ, Pickles T, Hood K, et al. Personalised risk communication for informed decision making about taking screening tests. Cochrane Database of Systematic Reviews 2013, Issue 2. [DOI: 10.1002/14651858.CD001865.pub3] 


\section{Ell 2010}

Ell K, Katon W, Xie B, Lee PJ, Kapetanovic S, Guterman J, et al. Collaborative care management of major depression among low-income, predominantly Hispanic subjects with diabetes: a randomised controlled trial. Diabetes Care. 2010/01/26 2010; Vol. 33, issue 4:706-13.

Elwyn 2012a

Elwyn G, Lloyd A, Joseph-Williams N, Cording E, Thomson R, Durand MA, et al. Option Grids: shared decision making made easier. Patient Education and Counseling 2012;90(2):207-12. [DOI: 10.1016/ j.pec.2012.06.036]

\section{Elwyn 2012b}

Elwyn G, Frosch D, Thomson R, Joseph-Williams N, Lloyd A, Kinnersley P, et al. Shared decision making: a model for clinical practice. Journal of General Internal Medicine 2012; 27(10):1361-7.

Elwyn 2014

Elwyn G, Dehlendorf C, Epstein RM, Marrin K, White J, Frosch DL. Shared decision making and motivational interviewing: achieving patient-centred care across the spectrum of health care problems. Annals of Family Medicine 2014;12(3):270-5.

\section{Entwistle 2012}

Entwistle VA, Cribb A, Watt IS. Shared decision making: enhancing the clinical relevance. Journal of the Royal Society of Medicine 2012;105(10):416-21.

Entwistle 2013

Entwistle VA, Cribb A. Enabling People to Live Well. London: Health Foundation, 2013.

Epping-Jordan 2004

Epping-Jordan JE, Pruitt SD, Bengoa R, Wagner EH. Improving the quality of health care for chronic conditions. Quality and Safety in Health Care. 2004/08/04 2004; Vol. 13 , issue 4:299-305.

\section{First 1997}

First M, Spitzer R, Gibbon M, Williams JBW. Structured Clinical Interview for DSM-IV Axis I Disorders (SCID-I), clinician version. Washington, DC: American Psychiatric Press, 1997.

\section{Fitzgerald 1998}

Fitzgerald JT, Anderson RM, Funnell MM, Hiss RG, Hess GE, Davis WK, et al. The reliability and validity of a brief diabetes knowledge test. Diabetes Care 1998;21(5):706-10.

Follmann 1992

Follmann D, Elliott P, Suh I, Cutler J. Variance imputation for overviews of clinical trials with continuous response. Journal of Clinical Epidemiology 1992;45(7):769-73.

\section{Forder 2012}

Forder J, Jones K, Glendinning C, Caiels J, Welch E, Baxter $\mathrm{K}$, et al. Evaluation of the personal health budget pilot programme. http://www.personalhealthbudgets.dh.gov.uk/ - library/Resources/Personalhealthbudgets/2012/ PHBE personal 'health 'budgets 'final' report 'Nov' 2012.pdf. Canterbury: University of Kent, 2012 (accessed 21 Dec 2012).

\section{Foster 2007}

Foster G, Taylor S J, Eldridge S E, Ramsay J, Griffiths C J. Self-management education programmes by lay leaders for people with chronic conditions. Cochrane Database of Systematic Reviews 2007, Issue 4. [DOI: 10.1002/ 14651858.CD005108.pub2]

\section{Franek 2013}

Franek J. Self-management support interventions for persons with chronic disease: an evidence-based analysis. Ontario Health Technology Assessment Series 2013;13(9): $1-60$.

\section{George Institute 2011}

George Institute for Global Health. Realising the Potential of Patient Empowerment for Tackling Chronic Disease: Moving from Concept to Business as Usual. www.bupa.com/media/289194/ realising the potential ${ }^{\prime}$ of patient ${ }^{~}$ empowerment ${ }^{\prime}-$ final.pdf $^{\prime}$ 2011 (accessed 21 Dec 2012).

\section{Glasgow 2004a}

Glasgow RE, Nutting PA, King DK, Nelson CC, Cutter G, Gaglio B, et al. A practical randomized trial to improve diabetes care. Journal of General Internal Medicine 2004a;19 (12):1167-74.

\section{Glasgow 2005b}

Glasgow RE, Whitesides H, Nelson CC, King DK. Use of the Patient Assessment of Chronic Illness Care (PACIC) with diabetic patients: relationship to patient characteristics, receipt of care, and self-management. Diabetes Care 2005b; 28(11):2655-61.

\section{Graffy 2009}

Graffy J, Eaton S, Sturt J, Chadwick P. Personalized care planning for diabetes: policy lessions from systematic reviews of consultation and self-management interventions. Primary Health Care Research \& Development 2009;10: 210-222.

\section{Guy 1976}

Guy W. ECDEU Assessment Manual for Psychopharmacology. Rockville, MD: Department of Health, Education and Welfare, National Institute of Mental Health, 1976.

\section{Hibbard 2004}

Hibbard JH, Stockard J, Mahoney ER, Tusler M. Development of the Patient Activation Measure (PAM): conceptualizing and measuring activation in patients and consumers. Health Services Research 2004; Vol. 39, issue 4 Pt 1:1005-26.

\section{Hibbard 2005}

Hibbard JH, Mahoney ER, Stockard J, Tusler M. Development and testing of a short form of the patient activation measure. Health Services Research 2005;40(6): 1918-30.

Higgins 2011

Higgins JPT, Green S (editors). Cochrane Handbook for Systematic Reviews of Interventions Version 5.1.0 [updated March 2011]. The Cochrane Collaboration, 2011. Available from www.cochrane-handbook.org. 


\section{Howie 1998}

Howie J, Heaney D, Maxwell M, Walker J. A comparison of Patient Enablement Instrument (PEI) against two established satisfaction scales as an outcome measure of primary care consultations. Family Practice 1998;15(2): $165-71$.

\section{Jordan 2007}

Jordan JE, Osborne RH. Chronic disease self-management education programs: challenges ahead. Medical Journal of Australia 2007;186(2):84-7.

\section{Juniper 1992}

Juniper EF, Guyatt GH, Epstein RS, Ferrie PJ, Jaeschke R, Hiller TK. Evaluation of impairment of health-related quality of life in asthma: development of a questionnaire for use in clinical trials. Thorax 1992;47(2):76-83.

\section{Juniper 1999}

Juniper EF, Guyatt GH, Cox FM, Ferrie PJ, King D. Development and validation of the Mini Asthma Quality of Life Questionnaire. Eur Respir J 1999;14(1):32-8.

\section{Katon 2010b}

Katon W, Lin EH, Von Korff M, Ciechanowski P, Ludman $\mathrm{E}$, Young B, et al. Integrating depression and chronic disease care among patients with diabetes and/or coronary heart disease: the design of the TEAMcare study. Contemporary Clinical Trials 2010b;31(4):312-22.

\section{Kennedy 2005}

Kennedy A, Gask L, Rogers A. Training professionals to engage with and promote self-management. Health Education Research. 2005/03/03 2005; Vol. 20, issue 5: $567-78$.

\section{Kennedy 2014}

Kennedy A, Rogers A, Chew-Graham C, Blakeman T, Bowen R, Gardner C, Lee V, Morris R, Protheroe J. Implementation of a self-management support approach (WISE) across a health system: a process evaluation explaining what did and did not work for organisations, clinicians and patients. Implementation Science 2014;9:129.

\section{Kind 1996}

Kind P. The Euro-Qol instrument: an index of healthrelated quality of life. In: Spilker B editor(s). Quality of Life and Pharmacoeconomics in Clinical Trials. 2nd Edition. Lippincott-Raven, 1996.

\section{Kinnersley 2007}

Kinnersley P, Edwards AGK, Hood K, Cadbury N, Ryan $\mathrm{R}$, Prout $\mathrm{H}$, et al. Interventions before consultations for helping patients address their information needs. Cochrane Database of Systematic Reviews 2007, Issue 3. [DOI: 10.1002/14651858.CD004565.pub2]

\section{Kiresuk 1968}

Kiresuk T J, Sherman R. Goal attainment scaling: a general method for evaluating comprehensive community mental health programs. Community Mental Health Journal 1968;4 (6):443-53

\section{Kroenke 2001}

Kroenke K, Spitzer R L, Williams J B W. The PHQ-9: validity of a brief depression severity measure. Journal of General Internal Medicine 2001;16(9):606-13.

\section{Lai 2010}

Lai DTC, Cahill K, Qin Y, Tang J-L. Motivational interviewing for smoking cessation. Cochrane Database of Systematic Reviews 2010, Issue 1. [DOI: 10.1002/ 14651858.CD006936.pub2]

\section{Legare 2014}

Legare F, Stacey D, Turcotte S, Cossi M-J, Kryworuchko J, Graham ID, et al. Interventions for improving the adoption of shared decision making by healthcare professionals. Cochrane Database of Systematic Reviews 2014, Issue 9. [DOI: 10.1002/14651858.CD006732.pub3]

\section{Leon 1997}

Leon AC, Ollson M, Portera L, Farber L, Sheehan DV. Assessing psychiatric impairment in primary care with the Sheehan disability scale. International Journal of Psychiatry in Medicine 1997;27(2):93-105.

Lev 1996

Lev E L, Owen S V. A measure of self-care self-efficacy. Research in Nursing \& Health 1996;19:421-9.

\section{Levack 2012}

Levack WMM, Siegert RJ, Dean SG, McPherson K, HaySmith EJC, Weatherall MM. Goal setting and activities to enhance goal pursuit for adults with acquired disabilities participating in rehabilitation. Cochrane Database of Systematic Reviews 2012, Issue 4. [DOI: 10.1002/ 14651858.CD009727]

\section{Leventhal 1998}

Leventhal H, Leventhal EA, Contrada RJ. Self-regulation, health and behavior: a perceptual-cognitive approach. Psychology and Health 1998;13:717-33.

Lorig 1996 Lorig K, Stewart AL, Ritter P, Gonzalez VM, Laurent D, Lynch J. Outcome Measures for Health Education and Other Health Care Interventions. Sage, 1996.

\section{Mangin 2012}

Mangin D, Heath I, Jamoulle M. Beyond diagnosis: rising to the multi-morbidity challenge. BMJ 2012;344:e3526. [DOI: 10.1136/bmj.e3526]

\section{Matza 2008}

Matza LS, Yu-Isenberg KS, Coyne KS, Park J, Wakefield J, Skinner E P, et al. Further testing of the reliability and validity of the ASK-20 adherence barrier questionnaire in a medical center outpatient population. Current Medical Research and Opinions 2008;24(11):3197-208.

May 2009

May C, Montori V, Mair F. We need minimally disruptive medicine. BMJ 2009;339:485-7.

\section{Michie 2003}

Michie S, Miles J, Weinman J. Patient-centredness in chronic illness: what is it and does it matter?. Patient Education and Counseling 2003;51(3):197-206. 


\section{Montori 2006}

Montori VM, Gafni A, Charles C. A shared treatment decision-making approach between patients with chronic conditions and their clinicians: the case of diabetes. Health Expectations 2006;9(1):25-36.

\section{Mulley 2012}

Mulley A, Trimble C, Elwyn G. Patients' preferences matter: stop the silent misdiagnosis. http://www.kingsfund.org.uk/ publications/patients $\%$ E2\%80\%99-preferences-matter 2012 (accessed 21 Dec 2012).

\section{Mundt 2002}

Mundt JC, Marks IM, Shear MK, Greist JH. The Work and Social Adjustment Scale: a simple measure of impairment in functioning. British Journal of Psychiatry 2002;180:461-4.

Murray 2005

Murray E, Burns J, See Tai S, Lai R, Nazareth I. Interactive Health Communication Applications for people with chronic disease. Cochrane Database of Systematic Reviews 2005, Issue 4. [DOI: 10.1002/ 14651858.CD004274.pub4]

\section{Newbould 2012}

Newbould J, Burt J, Bower P, Blakeman T, Kennedy A, Rogers A, et al. Experiences of care planning in England: interviews with patients with long term conditions. $B M C$ Family Practice 2012;13(1):71.

Nolte 2008

Nolte E, McKee M. Integration and chronic care: a review. In: Nolte E, McKee M editor(s). Caring for People with Chronic Conditions: A Health System Perspective. Maidenhead, UK: McGraw Hill, 2008:64-91.

\section{Pols 2008}

Pols RG, Battersby MW. Coordinated care in the management of patients with unexplained physical symptoms: depression is a key issue. Medical Journal of Australia 2008;188(12 Suppl):S133-7.

Powell 2002

Powell H, Gibson PG. Options for self-management education for adults with asthma. Cochrane Database of Systematic Reviews 2002, Issue 3. [DOI: 10.1002/ 14651858.CD004107]

Prochaska 1992

Prochaska JO, Diclemente CC. Stages of change in the modification of problem behaviors. Progress in Behavior Modification 1992;28:183-218.

Protheroe 2010

Protheroe J, Blakeman T, Bower P, Chew-Graham

C, Kennedy A. An intervention to promote patient participation and self-management in long term conditions: development and feasibility testing. BMC Health Services Research 2010;10:206. [DOI: 10.1186/1472-6963-10-206]

\section{Radloff 1977}

Radloff LS. The CES-D scale: a self-report depression scale for research in the general population. Applied Psychological Measurement 1977;1:385-401.

\section{Renders 2000}

Renders CM, Valk GD, Griffin S, Wagner EH, Van E, Assendelft WJJ. Interventions to improve the management of diabetes mellitus in primary care, outpatient and community settings. Cochrane Database of Systematic Reviews 2000, Issue 4. [DOI: 10.1002/14651858.CD001481]

Reuben 2012

Reuben DB, Tinetti ME. Goal-oriented patient care: an alternative health outcomes paradigm. New England Journal of Medicine 2012;366(9):777-9.

Ross 2004

Ross SE, Moore LA, Earnest MA, Wittevrongel L, Lin CT. Providing a web-based online medical record with electronic communication capabilities to patients with congestive heart failure: randomised trial. Journal of Medical Internet Research 2004;6(2):e12. [DOI: 10.2196/jmir.6.2.e12]

Rubak 2005

Rubak S, Sandbaek A, Lauritzen T, Christensen B. Motivational interviewing: a systematic review and metaanalysis. British Journal of General Practice 2005;55(513): 305-12.

\section{Ryan 2011}

Ryan R, Hill S, Prictor M, McKenzie J. Cochrane Consumers and Communication Review Group: Study Quality Guide. www.latrobe.edu.au/chcp/cochrane/ resources.html 2011 (accessed 21 December 2012). [: [accessed 21/12/12]]

Schillinger 2008 Schillinger D, Hammer H, Wang F, Palacios J, McLean I, Tang A, et al. Seeing in 3-D: examining the reach of diabetes self-management support strategies in a public health care system. Health Education \& Behavior 2008;35(5):664-82.

\section{Schmittdiel 2008}

Schmittdiel J, Mosen DM, Glasgow RE, Hibbard J, Remmers C, Bellows J. Patient Assessment of Chronic Illness Care (PACIC) and improved patient-centered outcomes for chronic conditions. Journal of General Internal Medicine 2008; Vol. 23, issue 1:77-80.

\section{Schoen 2011}

Schoen C, Osborn R, Squires D, Doty M, Pierson R, Applebaum S. New 2011 survey of patients with complex care needs in eleven countries finds that care is often poorly coordinated. Health Affairs. 2011/11/11 2011; Vol. 30, issue 12:2437-48.

\section{Schünemann 2011}

Schünemann HJ, Oxman AD, Higgins JPT, Vist GE, Glasziou P, Guyatt GH. Chapter 11: Presenting results and 'Summary of findings' tables. In: Higgins JPT, Green $S$ (editors). Cochrane Handbook for Systematic Reviews of Interventions Version 5.1.0 [Updated March 2011]. The Cochrane Collaboration, 2011. Available from www.cochrane-handbook.org.

\section{Shepherd 2011}

Shepherd HL, Barratt A, Trevena LJ, McGeechan K, Carey $\mathrm{K}$, Epstein RM, et al. Three questions that patients can ask 
to improve the quality of information physicians give about treatment options: a cross-over trial. Patient Education and Counseling 2011; Vol. 84, issue 3:379-85.

\section{Singh 2008}

Singh D. How Can Chronic Disease Management Programmes Operate Across Care Settings and Providers? . www.euro.who.int/“ data/assets/pdf' file/0009/75474/ E93416.pdf 2008 (accessed 16 January 2015).

\section{Smedslund 2011}

Smedslund G, Berg RC, Hammerstrøm KT, Steiro A, Leiknes KA, Dahl HM, et al. Motivational interviewing for substance abuse. Cochrane Database of Systematic Reviews 2011, Issue 5. [DOI: 10.1002/ 14651858.CD008063.pub2]

\section{Stacey 2014}

Stacey D, Legare F, Col NF, Bennett CL, Barry MJ, Eden KB, et al. Decision aids for people facing health treatment or screening decisions. Cochrane Database of Systematic Reviews 2014, Issue 1. [DOI: 10.1002/ 14651858.CD001431.pub4]

Steinsbekk 2012

Steinsbekk A, Rygg LØ, Lisulo M, Rise M B, Fretheim A. Group based diabetes self-management education compared to routine treatment for people with type 2 diabetes mellitus. A systematic review with meta-analysis. Health Services Research 2012;12:213.

Stellefson 2013

Stellefson M, Dipnarine K, Stopka C. The Chronic Care Model and diabetes management in US primary care settings: a systematic review. Preventing Chronic Disease 2013;10:120180.

Tomich 2004

Tomich PL, Helgeson VS. Is finding something good in the bad always good? Benefit finding among women with breast cancer. Health Psychology 2004;23(1):16-23.

Tsai 2005

Tsai AC, Morton SC, Mangione CM, Keeler EB. A metaanalysis of interventions to improve care for chronic illnesses. American Journal of Managed Care 2005;11(8): 478-88.

\section{UN Secretary General 2011}

UN Secretary General. Prevention and control of noncommunicable diseases. www.ghdnet.org/sites/default/files/UN\%20SecretaryGeneral's\%20Report\%20on\%20NCDs.pdf 2011 (accessed 16 January 2015); Vol. Sixty-sixth session.

\section{Ustun 2010}

Ustun TB, Chatterji S, Kostanjsek N, Rehm J, Kennedy C, Epping-Jordan J, et al. Developing the World Health Organization Disability Assessment Schedule 2.0. Bulletin of the World Health Organization 2010;88:815-23.

Van der Bijl 1999

Van der Bijl J, Van Poelgeest-Eeltink A, Shortridge-Baggett L. The psychometric properties of the diabetes management self-efficacy scale for patients with type 2 diabetes mellitus. Journal of Advanced Nursing 1999;30(2):352-9.

\section{Von Korff 1997}

Von Korff M, Gruman J, Schaefer J, Curry SJ, Wagner EH. Collaborative management of chronic illness. Annals of Internal Medicine 1997;127(12):1097-102.

\section{Wagner 1998}

Wagner EH. Chronic disease management: what will it take to improve care for chronic illness?. Effective Clinical Practice 1998;1(1):2-4.

\section{Wanless 2002}

Wanless D. Securing Our Future Health: Taking a Long-Term View. London: HM Treasury, 2002.

\section{Ware 1992}

Ware JE, Sherbourne CD. The MOS short-form health survey (SF-36): I: Conceptual framework and item selection. Medical Care 1992;30(3):473-83.

\section{Ware 1996}

Ware JE, Kosinski M, Keller SD. A 12-item short form health survey: construction of scales and preliminary tests of reliability and validity. Medical Care 1996;34(3):220-33.

\section{Welch 1997}

Welch GW, Jacobson AM, Polonsky WH. The problem areas in diabetes scale. Diabetes Care 1997;20(5):760-6.

Wensing 2003

Wensing M, Vingerhoets E, Grol R. Feedback based on patient evaluations: a tool for quality improvement? Patient Education and Counseling 2003; Vol. 51, issue 2: 149-53.

\section{Wetzels 2007}

Wetzels R, Harmsen M, Van Weel C, Grol R, Wensing M. Interventions for improving older patients' involvement in primary care episodes. Cochrane Database of Systematic Reviews 2007, Issue 1. [DOI: 10.1002/ 14651858.CD004273.pub2]

\section{Williams 1998}

Williams GC, Freeman ZR, Deci EL. Supporting autonomy to motivate patients with diabetes for glucose control. Diabetes Care 1998;21(10):1644-51.

\section{Williams 2011a}

Williams AM, Dennis S, Harris MF. How effective are the linkages between self-management programmes and primary care providers, especially for disadvantaged patients?. Chronic Illness 2011;7(1):20-30.

Williams 2011b

Williams B, Steven K, Sullivan FM. Tacit and transitionary: an exploration of patients' and primary care health professionals' goals in relation to asthma. Social Science \& Medicine 2011;72(8):1359-66.

\section{Woltmann 2012}

Woltmann E, Grogan-Kaylor A, Perron B, Georges H, Kilbourne A, Bauer MS. Comparative effectiveness of collaborative chronic care models for mental health conditions across primary, specialty, and behavioral health 
care settings: systematic review and meta-analysis. American Journal of Psychiatry 2012;169(8):790-804.

World Health Organization 2012

World Health Organization. Chronic diseases.

www.who.int/topics/chronic diseases/en/ (accessed 21 Dec 2012).

Year of Care 2011

Year of Care Programme. Report of findings from the pilot programme. www.diabetes.org.uk/upload/Professionals/ Year\%20of\%20Care/YOC 'Report.pdf 2011 (accessed 16 January 2015).

* Indicates the major publication for the study 


\section{CHARACTERISTICS OF STUDIES}

\section{Characteristics of included studies [ordered by study ID]}

Battersby 2007

Methods

Participants

Study design: 8 studies in 4 regions, including 4 RCTs (the others used geographic controls)

Unit of randomisation: patient

Unit of analysis: patient

Funding sources: South Australian Health Commission; Commonwealth Department of Health and Aged Care

Conflicting interests: none declared

\section{Country: Australia}

Setting: Primary care

Conditions/numbers: 1703 patients with various conditions: Central region: cardiac (n = 271 intervention, 138 control); Southern region: respiratory $(n=165$ intervention, 62 control), somatisation ( $\mathrm{n}=90$ intervention, 35 control), aged care $(\mathrm{n}=632$ intervention, 310 control) - total 1158 intervention, 545 control. (Battersby 2005 p. 663)

\section{Health literacy: $n / a$}

Multi-morbidity: n/a

Interventions
Theoretical framework: Chronic Care Model

Focus: Both clinician and patient

Type of intervention: Structured, face-to-face planning and care co-ordination + staff training

Clinicians involved: Service co-ordinators (nurses + allied health professionals - additional) and regular GP

Tools: SA HealthPlus Co-ordinated Care. Service co-ordinator assisted the GP to develop a care plan based on a care plan generator. This included patient's self-defined problems and goals. Based on this and the GP's knowledge of the patient, as well as the patient's 'Problems \& Goals' statements, the GP and patient made a joint decision on what support and services were needed. Both the GP and the patient signed the care plan, and copies were made for patient, service co-ordinator, other providers and GP. Service co-ordinators received 2 days training + competency assessment and group supervision. The service co-ordinator helped the patient gain access to and co-ordinate community and patient education services and worked with the patient to achieve his or her goals. GPs were paid a fee to develop each care plan and an annual fee to oversee patients' care, supported by the service co-ordinators

Stages completed: Extended - A, B,C,D,E,F,G

Usual provider aware of patient's goals and action plans: Yes

Standardisation of clinician input: Good (training and ongoing supervision), but "The [intervention group] GPs needed reminders to order the services scheduled on the care plan.” (p. 62)

Fidelity: Weak. "The intervention was not in place long enough for its full implementation" (p. 62)

Attrition: High. More than $50 \%$ of participants lost to follow-up following trial extension (p. 48)

Comparison: Usual care 
Battersby 2007 (Continued)

\begin{tabular}{|c|c|}
\hline Outcomes & $\begin{array}{l}\text { Health status: } \text { subjective: Medical Outcomes Study short form (SF-36), Work and Social } \\
\text { Adjustment Scale (WSAS); psychological: Beck Depression Inventory (BDI), State-Trait } \\
\text { Anxiety Inventory (STAI), Hostility and Direction of Hostility Questionnaire (HDHQ) } \\
\text { (Pols 2008) } \\
\text { Self-management capabilities: n/a } \\
\text { Health behaviours: n/a } \\
\text { Attainment of personal goals: problems and goals score } \\
\text { Service use: *service use and costs } \\
\text { Adverse events: none reported } \\
\text { Timing of outcome measures: } 12 \text { months; baseline measures not reported }\end{array}$ \\
\hline Notes & $\begin{array}{l}\text { *Primary outcome. Negative results not reported in full. Author contacted - more papers } \\
\text { supplied but no relevant additional data obtained. Data from one sub-trial with complete } \\
\text { results (Pols 2008) included in meta-analysis. Pols 2008: power calculation - required } \\
\text { sample size of } 300 \text { participants to detect } 15 \% \text { reduction in hospital admissions but only } \\
124 \text { recruited, so under-powered }\end{array}$ \\
\hline
\end{tabular}

Risk of bias

\begin{tabular}{lll}
\hline Bias & Authors' judgement & Support for judgement \\
\hline $\begin{array}{l}\text { Random sequence generation (selection } \\
\text { bias) }\end{array}$ & Low risk & $\begin{array}{l}\text { Pols 2008: randomisation performed by } \\
\text { random number allocation (p. S133) }\end{array}$ \\
\hline
\end{tabular}

Allocation concealment (selection bias) Low risk

Pols 2008: random number allocation provided to the research officer by telephone from the local evaluation team (p. S133)

Blinding of participants and personnel Unclear risk (performance bias)

All outcomes

Pols 2008: GPs were not blinded to participant allocation. All GPs looked after participants in both intervention and control groups, (p. S133)

Blinding of outcome assessment (detection Low risk bias)

All outcomes
Pols 2008: research officers were not blinded to participant allocation, but outcome assessments were administered independently by separate contractors using postal questionnaires mailed to participants (p. S134)

Incomplete outcome data (attrition bias) High risk All outcomes
Pols 2008: High levels of attrition: only 42 out of 89 in the intervention group (47\%) and 22 of 44 in the control group (50\%) completed the study. Reasons for loss to follow-up reported for study as a whole but not for the two randomised sub-trials, so not possible to isolate these. Service use analysed on an intention-to-treat basis but 
Battersby 2007 (Continued)

not possible for SF-36 and WSAS

\begin{tabular}{|c|c|c|}
\hline Selective reporting (reporting bias) & High risk & $\begin{array}{l}\text { Battersby } 2007 \text { reports significant results } \\
\text { only for SF-36 and WSAS, not non-signif- } \\
\text { icant findings, and RCTs and those with } \\
\text { geographical controls are lumped together. } \\
\text { Battersby } 2005 \text { reports only significant re- } \\
\text { sults for SF-36, not total scores or non-sig- } \\
\text { nificant results for subscales. Cost data in- } \\
\text { clude only those who had an inpatient ad- } \\
\text { mission prior to entry. These facts are made } \\
\text { clear in the papers. Pols } 2008 \text { reports full } \\
\text { results for SF-36 but not for WSAS, but } \\
\text { they state that there was no significant dif- } \\
\text { ference in results for WSAS (p. S136) }\end{array}$ \\
\hline Other bias & Unclear risk & $\begin{array}{l}\text { Work and Social Adjustment Scale has not } \\
\text { been validated in a chronically ill popula- } \\
\text { tion and the work questions were omitted } \\
\text { because not relevant to most participants } \\
\text { (p. } 46 \text { ) }\end{array}$ \\
\hline
\end{tabular}

Frosch 2011

Methods

Study design: RCT

Unit of randomisation: patient

Unit of analysis: patient

Funding sources: Robert Wood Johnson Foundation; Foundation for Informed Medical Decision Making; National Institute on Aging; National Institutes of Health

Conflicting interests: fees/grants from Foundation for Informed Medical Decision Making

\begin{tabular}{|c|c|}
\hline Participants & $\begin{array}{l}\text { Country: USA } \\
\text { Setting: Primary care } \\
\text { Conditions/numbers: } 201 \text { diabetes patients (type } 2) \text { (100 intervention, } 101 \text { control) } \\
\text { Health literacy: Predominantly poor, uninsured ethnic-minority patients with poorly } \\
\text { controlled diabetes (p. } 2015) \\
\text { Multi-morbidity: Charlson co-morbidity index - intervention } 0.81 \pm 1.3 \text {, control } 0.66 \\
\pm 1.2\end{array}$ \\
\hline Interventions & $\begin{array}{l}\text { Theoretical framework: n/a } \\
\text { Focus: patient } \\
\text { Type of intervention: Information + structured coaching (phone) } \\
\text { Clinicians involved: Health coach (additional) } \\
\text { Tools: Participants viewed a } 24 \text {-minute-long DVD plus booklet, followed by up to } 5 \\
\text { sessions of telephone coaching with a trained nurse educator. First session was up to } 60 \\
\text { minutes, second and third } 30 \text { minutes, fourth and fifth } 15 \text { minutes. Maximum amount of } \\
\text { coaching time was } 2 \frac{1}{2} 2 \text { hours. Purpose was to collaborate with participants in identifying } \\
\text { desired and attainable behavioural goals that could have a positive impact on their diabetes }\end{array}$ \\
\hline
\end{tabular}

Personalised care planning for adults with chronic or long-term health conditions (Review)

Copyright (c) 2015 The Cochrane Collaboration. Published by John Wiley \& Sons, Ltd. 
Frosch 2011 (Continued)

management. The coach collaborated with participants to develop a specific behavioural plan, which was then monitored and adjusted as participants attempted to implement their behavioural goals. A single health coach trained in patient-centred approaches to diabetes management and motivational enhancement saw all participants. Participants received a call 1 week after enrolment to remind them to review the brochure and DVD. They were eligible to receive up to 5 sessions of telephone coaching, but there were frequent delays in contact and only $73 \%$ completed 5 coaching sessions

Stages completed: Limited - B, C, F

Usual provider aware of patient's goals and action plans: Not stated

Standardisation of clinician input: A single trained clinician provided all coaching sessions.

Fidelity: $73 \%$ completed 5 coaching sessions, $15 \%$ did not complete any

Attrition: 5\% intervention, 14\% control lost to follow-up

Comparison: Usual care + booklet

Outcomes

Health status: physical: blood glucose (HbA1c)*, cholesterol, blood pressure, BMI

Self-management capabilities: University of Michigan Diabetes Knowledge Test

Health behaviours: 25 -item Summary of Diabetes Self-Care Activities measure (diet, exercise, blood glucose testing, foot care, smoking), adherence to medications

Achievement of personal goals: $\mathrm{n} / \mathrm{a}$

Service use: $\mathrm{n} / \mathrm{a}$

Adverse events: none reported

Timing of outcome measures: Baseline, 1 month, 6 months

Notes

*Primary outcome. Power calculation - required sample size of 200 participants to detect meaningful difference between the groups on HbAlc

Risk of bias

\begin{tabular}{|c|}
\hline Bias \\
\hline
\end{tabular}

Random sequence generation (selection Unclear risk bias)

Participants were randomised into equally sized control and experimental conditions using a predetermined randomisation sequence concealed in sealed envelopes (p. 2012)

\begin{tabular}{|c|c|c|}
\hline Allocation concealment (selection bias) & Low risk & Sealed envelopes. \\
\hline $\begin{array}{l}\text { Blinding of participants and personnel } \\
\text { (performance bias) } \\
\text { All outcomes }\end{array}$ & Low risk & $\begin{array}{l}\text { Not possible to blind participants, but as } \\
\text { most outcomes were objective it is unlikely } \\
\text { to have a significant effect on risk of bias }\end{array}$ \\
\hline $\begin{array}{l}\text { Blinding of outcome assessment (detection } \\
\text { bias) } \\
\text { All outcomes }\end{array}$ & Low risk & $\begin{array}{l}\text { Research staff were not blinded to partic- } \\
\text { ipants' assignments, but primary outcome } \\
\text { was biological measure that is not sensitive } \\
\text { to unblinding (p. 2016) }\end{array}$ \\
\hline
\end{tabular}

Personalised care planning for adults with chronic or long-term health conditions (Review) 
Frosch 2011 (Continued)

\begin{tabular}{|c|c|c|}
\hline $\begin{array}{l}\text { Incomplete outcome data (attrition bias) } \\
\text { All outcomes }\end{array}$ & Low risk & $\begin{array}{l}84 \% \text { completed the } 6 \text {-month survey (p. } \\
\text { 2013). Intention-to-treat analysis reported } \\
\text { with missing data imputed }\end{array}$ \\
\hline Selective reporting (reporting bias) & Unclear risk & No published protocol. \\
\hline Other bias & Low risk & $\begin{array}{l}\text { Randomisation occurred after participants } \\
\text { completed their medical consultations to } \\
\text { mask healthcare providers to participants } \\
\text { assignment (P. 2012) }\end{array}$ \\
\hline
\end{tabular}

\section{Glasgow 2005a}

Methods

Study design: Cluster-RCT

Unit of randomisation: physician

Unit of analysis: patient

Funding sources: Agency for Health Research and Quality

Conflicting interests: none declared

$\begin{array}{ll}\text { Carticipants } & \text { Country: USA } \\ & \text { Setting: Primary care } \\ & \text { Conditions/numbers: } 886 \text { diabetes patients (type 2) (469 intervention, } 417 \text { control) } \\ \text { Health literacy: } n / a \\ \text { Multi-morbidity: } n / a\end{array}$

Interventions

Theoretical framework: Chronic Care Model

Focus: patient

Type of intervention: Information + self-management support (phone or face-to-face) Clinicians involved: Care manager (additional)

Tools: Diabetes Priority Program. Participants were asked to come 30 minutes early to 2 diabetes-related visits, scheduled 6 months apart, to complete a computerised assessment and action-planning procedure. The CD-Rom-assisted diabetes care enhancement program with touchscreen assessment and feedback to check receipt of lab tests and other clinical procedures (NCQ/ADA Diabetes Physician Recognition Program - PRP) and self-management support, and to develop a self-management action plan focusing on behaviour change in diet, smoking and physical exercise involving personal goals. Three printouts summarised results for participant, physician and care manager (nurse or medical assistant), including prominent notation of areas the participant wished to discuss. Care managers trained in patient-centred self-management support met with participants or scheduled phone calls and organised follow-ups to review progress. The discussion included review of the medical care needs and self-care goals that the participant identified and brainstorming additional strategies that participants could use to overcome barriers to their goals. This took an average of $8-10$ minutes. The care manager also attempted a brief follow-up call after each visit to review progress and to reinforce strategies developed. These procedures were repeated at the next visit about 6 months later

Stages completed: Limited - B, C, D, F 
Usual provider aware of patient's goals and action plans: Yes

Standardisation of clinician input: Care managers received brief training only, none for physicians

Fidelity: $75 \%$ of eligible patients participated, $73 \%$ discussed print-out with physicians, $77 \%$ met care manager, $67 \%$ received at least 1 phone follow-up

Attrition: 19\% intervention, 15\% control lost to follow-up

Comparison: Completion of touch-screen computer assessment with PRP measures + general health risk appraisal + same number of visits + printout on general health risks, without PRP and follow-up calls

Outcomes

Health status: physical: HbA1c, cholesterol; psychological: Patient Health Questionnaire (PHQ-9); subjective: Problem Areas in Diabetes (PAID-2),

Self-management capabilities: $\mathrm{n} / \mathrm{a}$

Health behaviours: $n / a$

Achievement of personal goals: self report

Service use: $\mathrm{n} / \mathrm{a}$

Adverse events: none reported

Timing of outcome measures: Baseline, 12 months

Notes

Primary outcome - PRP measures (performance of specified clinical procedures) were excluded because not relevant to this review. Power calculation - required sample size of

32 physicians and 774 patients to detect a moderate effect

\section{Risk of bias}

Bias

Authors' judgement

Support for judgement

Random sequence generation (selection Low risk bias)

Participating physicians were stratified by size of practice and urban/rural setting. Randomisation was conducted by the project statistician, who then notified research staff of condition assignment

Allocation concealment (selection bias) Unclear risk Not reported.

Blinding of participants and personnel Unclear risk (performance bias)

Blinding not described. Not possible to

All outcomes

blind participants.

Blinding of outcome assessment (detection Unclear risk

bias)

Not reported.

All outcomes

Incomplete outcome data (attrition bias) Low risk All outcomes

Selective reporting (reporting bias)
Unclear risk
Attrition rates were approximately equivalent (19\% intervention and 15\% control). Analyses were conducted on complete cases

No published protocol. 
Other bias

Low risk
No evidence of selective recruitment of clusters. Participating physicians were stratified by size of practice and urban/rural setting. No significant differences between groups at baseline. To avoid contamination, all physicians within a given clinic were assigned to the same condition. To account for clustering of patients within physician. a generalised regression model using a random effect for the physician (a mixed model) was fitted, adjusting for baseline score on the dependent variable with a random physician effect and participants nested within physician (Glasgow 2004a, p. 1168). Outcomes were evaluated using mixed-model regression analyses (to account for clustering) and controlling for baselines scores on the dependent variable and any other potential confounding variables

Hart 1978

\begin{tabular}{|l|l} 
Methods & Study design: RCT \\
& Unit of randomisation: patient \\
& Unit of analysis: patient \\
& Funding sources: not stated \\
Conflicting interests: not stated
\end{tabular}

Participants

Country: USA

Setting: Community mental health centre

Conditions/numbers: 32 mental health patients (diagnoses unspecified) (16 intervention, 16 control)

Health literacy: $n / a$

Multi-morbidity: n/a

Interventions

Theoretical framework: $\mathrm{n} / \mathrm{a}$

Focus: patient

Type of intervention: Individual therapy

Clinicians involved: Clinician 'scaler' (additional) and psychotherapist (usual)

Tools: The Behavioral Monitoring Process Record (BMPR) was designed to help participants set goals and report on their progress at each subsequent therapy session. A 4week goal was set with the participant and reviewed each week. Within each problem area, a weekly goal and method of attainment was specified. Participant and therapist jointly assessed the degree of attainment of each goal. Goals had to be observable, definable and measurable and structured in a step-by-step manner. All participants completed an 'intake history' based on two interview sessions. At a third interview session they collaboratively prepared a follow-up guide that consisted of setting treatment goals

Personalised care planning for adults with chronic or long-term health conditions (Review)

Copyright $\Subset 2015$ The Cochrane Collaboration. Published by John Wiley \& Sons, Ltd. 
Hart 1978 (Continued)

and predicting 5 levels of goal attainment with an 'expected' level of attainment by the eighth therapy session. 'Collateral persons' (other people significant to the participant, such as spouse or probation officer) helped to identify problem areas and to validate the participant's self report at follow-up. Randomisation took place after the third session

Stages completed: Limited - B, C, D, F

Usual provider aware of patient's goals and action plans: Yes

Standardisation of clinician input: Only 4 therapists involved

Fidelity: Not reported

Attrition: None reported

Comparison: Same individual therapy without weekly goal setting or monitoring

$\begin{array}{ll}\text { Outcomes } & \text { Health status: } \mathrm{n} / \mathrm{a} \\ & \text { Self-management capabilities: } \mathrm{n} / \mathrm{a} \\ & \text { Health behaviours: } \mathrm{n} / \mathrm{a} \\ \text { Achievement of personal goals: Goal Attainment Scale (GAS) (Kiresuk 1968) - achieve- } & \text { ment of personal goals } \\ & \text { Service use: } \mathrm{n} / \mathrm{a} \\ \text { Adverse events: none reported } \\ \text { Timing of outcome measures: Baseline, } 3 \text { months }\end{array}$

\section{Risk of bias}

\begin{tabular}{|c|c|c|}
\hline Bias & Authors' judgement & Support for judgement \\
\hline $\begin{array}{l}\text { Random sequence generation (selection } \\
\text { bias) }\end{array}$ & Unclear risk & No details provided. \\
\hline Allocation concealment (selection bias) & Unclear risk & No details provided. \\
\hline $\begin{array}{l}\text { Blinding of participants and personnel } \\
\text { (performance bias) } \\
\text { All outcomes }\end{array}$ & Unclear risk & No details provided. \\
\hline $\begin{array}{l}\text { Blinding of outcome assessment (detection } \\
\text { bias) } \\
\text { All outcomes }\end{array}$ & Unclear risk & No details provided. \\
\hline $\begin{array}{l}\text { Incomplete outcome data (attrition bias) } \\
\text { All outcomes }\end{array}$ & Unclear risk & No details provided. \\
\hline Selective reporting (reporting bias) & Unclear risk & No published protocol. \\
\hline Other bias & Low risk & $\begin{array}{l}\text { The collateral person was a source of ex- } \\
\text { ternal validation of the participant's self } \\
\text { report. Validation included identification } \\
\text { and definition of the participant's problems } \\
\text { at intake (pretest score) and input as to }\end{array}$ \\
\hline
\end{tabular}


Hart 1978 (Continued)

the level of functioning on the attainment level of the follow-up guide at the followup evaluation

Hiss 2007

Methods

Study design: RCT

Unit of randomisation: patient

Unit of analysis: patient

Funding sources: National Institutes of Health; National Institute of Diabetes and

Digestive and Kidney Diseases

Conflicting interests: not stated

$\begin{array}{ll}\text { Participants } & \text { Country: USA } \\ & \text { Setting: Primary care } \\ & \text { Conditions/numbers: } 197 \text { diabetes patients (type 2) (95 intervention, } 102 \text { control) } \\ \text { Health literacy: recruited from community clinics serving under-insured residents } \\ \text { Multi-morbidity: n/a }\end{array}$

Interventions

Theoretical framework: $n / a$

Focus: patient

Type of intervention: Structured face-to-face meetings

Clinicians involved: Nurse care manager (additional) and usual primary care physician Tools: All participants received a comprehensive evaluation of their diabetes at the community clinic they attended. A report of the evaluation plus appropriate explanations and interpretations was mailed to both the participant and his or her physician. This preceded randomisation. Those in the intervention group then received several individually arranged meetings with a nurse care manager where they discussed problem identification, problem-specific, short-term goal setting and development of a tentative action plan. This was communicated to the primary care physician who participants were advised to contact to follow up identified problems. Then followed a collaborative interaction between nurse, physician, and participant focused on short-term goal attainment, plus proactive and continuous follow-up by the nurse care manager. Longterm goal setting typically occurred during subsequent nurse/participant meetings as the participant gained experience in carrying out the action plan. Short-and long-term goals were participant-specific and based on problems identified in the baseline evaluation. These included family issues, financial status, employment, insurance status, and access to and payment for medical care, as well as medical goals

Stages completed: Extended - A, B, C, E, F

Usual provider aware of patient's goals and action plans: Yes

Standardisation of clinician input: Single nurse care manager

Fidelity: In-person and phone contacts monitored and reported

Attrition: 15\% intervention, 19\% control lost to follow-up

Comparison: Usual care + evaluation of diabetes with report mailed to participant and physician

Outcomes

Health status: physical: HbA1c, serum cholesterol, systolic blood pressure (SBP), diastolic blood pressure (DBP)

Self-management capabilities: $\mathrm{n} / \mathrm{a}$

Personalised care planning for adults with chronic or long-term health conditions (Review)

Copyright @ 2015 The Cochrane Collaboration. Published by John Wiley \& Sons, Ltd. 
Hiss 2007 (Continued)

Health behaviours: $n / a$

Achievement of personal goals: $\mathrm{n} / \mathrm{a}$

Service use: $\mathrm{n} / \mathrm{a}$

Adverse events: none reported

Timing of outcome measures: Baseline, 6 months

Notes No power calculation reported.

Risk of bias

\begin{tabular}{|c|c|c|}
\hline Bias & Authors' judgement & Support for judgement \\
\hline $\begin{array}{l}\text { Random sequence generation (selection } \\
\text { bias) }\end{array}$ & Unclear risk & Not reported. \\
\hline Allocation concealment (selection bias) & Unclear risk & Not reported. \\
\hline $\begin{array}{l}\text { Blinding of participants and personnel } \\
\text { (performance bias) } \\
\text { All outcomes }\end{array}$ & Unclear risk & Not reported. \\
\hline $\begin{array}{l}\text { Blinding of outcome assessment (detection } \\
\text { bias) } \\
\text { All outcomes }\end{array}$ & Unclear risk & Not reported. \\
\hline $\begin{array}{l}\text { Incomplete outcome data (attrition bias) } \\
\text { All outcomes }\end{array}$ & Low risk & $\begin{array}{l}\text { All participants accounted for. Intervention } \\
\text { group: no post-intervention data obtained } \\
\text { from } 14 \text { participants }(\text { moved }=4 \text {, long-term } \\
\text { care }=1 \text {, lost }=7 \text {, refused }=2 \text { ). Control } \\
\text { group: no post-intervention data from } 19 \\
(\text { death }=3 \text {, moved }=3 \text {, long-term care }=1 \text {, } \\
\text { lost }=6 \text {, refused }=6)\end{array}$ \\
\hline Selective reporting (reporting bias) & Unclear risk & $\begin{array}{l}\text { No published protocol. All outcomes re- } \\
\text { ported, but no intention-to-treat analysis. } \\
\text { Missing BP data for } 3 \text { in intervention group } \\
\text { and } 2 \text { controls unaccounted for }\end{array}$ \\
\hline Other bias & Low risk & \\
\hline
\end{tabular}


Methods
Study design: RCT

Unit of randomisation: patient

Unit of analysis: patient

Funding sources: National Institute of Mental Health; Group Health Cooperative

Conflicting interests: fees/grants from Wyeth, Eli Lilly, Forest, Pfizer, Prescott Medical, HealthSTAR Communications, World Psychiatry Association, John A Hartford Foundation, Johnson \& Johnson, Samepage, Rewarding Health, Roche Diagnostics, Group Health Cooperative

\section{Country: USA}

Setting: Primary care

Conditions/numbers: 214 patients with depression + diabetes and/or CHD (106 intervention, 108 control)

Health literacy: $n / a$

Multi-morbidity: yes
Interventions

\section{Theoretical framework: $\mathrm{n} / \mathrm{a}$}

Focus: patient

Type of intervention: Structured, face-to-face self-management support + staff training Clinicians involved: Nurses (additional), primary care physicians (usual)

Tools: TEAMcare intervention combined support for self care with pharmacotherapy to control depression, hyperglycemia, hypertension, and hyperlipidemia. Participants worked collaboratively with nurses and primary care physicians to establish individualised clinical and self-care goals. In structured visits at each participant's primary care clinic every 2 to 3 weeks, nurses monitored participant's progress in management of depression, control of medical disease, and self-care activities. Treatment protocols guided adjustments of commonly used medicines in participants who did not achieve specific goals. Nurses followed participants proactively to provide support for medication adherence. Using motivational interviewing and coaching, nurses helped participants solve problems and set goals for improved medication adherence and self care (e.g. exercising and self-monitoring blood pressure and glucose levels). Participants received self-care materials including The Depression Handbook, a video compact disk on depression care, a booklet and other materials on chronic condition management and self-monitoring devices (e.g. blood pressure or blood glucose meters) appropriate to their condition. Nurses received weekly supervision with a psychiatrist, primary care physician, and psychologist to review new cases and participant progress. Supervising physicians recommended initial choices and changes in medications tailored to the participant's history and clinical response. When targeted levels were reached, the nurse and the participant developed a maintenance plan that included stress reduction, behavioural goals, continued use of medications, and identification of prodromal symptoms associated with worsening depression and glycemic control. Nurses then followed up with telephone calls every 4 weeks. Participants with disease control that worsened were offered follow-up visits or telephone calls and protocol-based intensification of treatment regimens

Stages completed: Extended - A, B, C, D, E, F, G

Usual provider aware of patient's goals and action plans: Yes

Standardisation of clinician input: strong - 2-day training course attended by the 3 study nurses + educational materials + weekly case-load reviews with physicians + close monitoring

Fidelity: Data and safety monitoring board, numbers of in-person and phone contacts 
monitored and reported

Attrition: $12 \%$ at 6 months and $17 \%$ at 12 months lost to follow-up

Comparison: Enhanced usual care

Outcomes

Health status: physical: HbA1c, systolic blood pressure, LDL cholesterol; psychological: Symptom Checklist-20; Patient Global Rating of Improvement*; subjective: Sheehan social role disability scale, WHO Disability Assessment Schedule (WHODAS-2), quality of life (0 to 10$)$ (NV)

Self-management capabilities: 4 selected questions from short-form Patient Activation Measure (PAM-13)

Health behaviours: diet, exercise, medication adherence

Achievement of personal goals: $\mathrm{n} / \mathrm{a}$

Service use: healthcare costs and cost effectiveness, including depression-free days, QALYs, outpatient costs

Adverse events: hospitalisations - 27 intervention, 23 control; deaths: 1 intervention, 2 control

Timing of outcome measures: Baseline, 6 months, 12 months

*Primary outcome. Power calculation - 290 participants required to detect a clinically significant difference in SCL-20 depression scores, HbA1c, systolic blood pressure and LDL cholesterol. Only 214 recruited so under-powered

\section{Risk of bias}

\begin{tabular}{|c|c|c|}
\hline Bias & Authors' judgement & Support for judgement \\
\hline $\begin{array}{l}\text { Random sequence generation (selection } \\
\text { bias) }\end{array}$ & Low risk & $\begin{array}{l}\text { Participants were randomly assigned by } \\
\text { computer using a permuted block design } \\
\text { with randomly selected block sizes of } 4,6 \text {, } \\
\text { and } 8 \text { patients }\end{array}$ \\
\hline
\end{tabular}

Allocation concealment (selection bias) Low risk

Research assistants who were unaware of the intervention status implemented study procedures (Katon 2010a)

Blinding of participants and personnel Unclear risk (performance bias)

All outcomes
Single-blind only - not possible to blind participants to intervention, but most outcomes objective so unlikely to affect risk of bias

Clinicians were not blinded to outcome assessments because these were part of the intervention

bias)

All outcomes

Incomplete outcome data (attrition bias) Low risk All outcomes
Fully detailed in Figure 1 of Katon 2010a 
Katon 2010a (Continued)

\begin{tabular}{l|l|l} 
Selective reporting (reporting bias) & Low risk & $\begin{array}{l}\text { Protocol published (Katon 2010b). Data } \\
\text { and safety monitoring board reviewed } \\
\text { methods and outcomes every } 6 \text { months. } \\
\text { Katon 2010a includes description of pre- } \\
\text { analysis modifications to protocol }\end{array}$ \\
\hline Other bias & Low risk & $\begin{array}{l}\text { Intervention provided by research nurses } \\
\text { not involved with control group, in collab- } \\
\text { oration with primary care physicians }\end{array}$ \\
\hline
\end{tabular}

Kennedy 2013

Methods

Study design: cluster-RCT

Unit of randomisation: general practices

Unit of analysis: patient

Funding sources: National Institute for Health Research; National Primary Care Research and Development Centre

Conflicting interests: none declared

Country: UK
Setting: Primary care
Conditions/numbers: 5599 patients with diabetes, COPD, or irritable bowel syndrome
from 43 practices (2295 intervention, 3304 control)
Multi-morbidity: n/a
Health literacy: Recruited from practices with high levels of socio-economic deprivation
(p. 2$)$

Interventions

Theoretical framework: Chronic Care Model, Normalisation Process Theory

Focus: Both clinician and patient

Type of intervention: Structured face-to-face coaching + staff training

Clinicians involved: Nurse (usual), GP (usual)

Tools: Whole System Informing Self-Management Engagement (WISE). The intervention was intended to be feasible to implement widely in primary care, which put practical limitations on the intensity of the intervention. Aim was to take several components and deliver them as a comprehensive package under naturalistic conditions using routine care providers to maximise real-world applicability. Two training sessions were organised for practice staff covering ways of embedding self-management tools in practice systems (session 1) and using core self-management skills in consultations and ensure participants received, or were directed to, appropriate resources (session 2). Fidelity checks and reinforcement sessions were scheduled after training. Two facilitators delivered the training and provided access to self-management support activities and resources. These included a tool to assess patient support needs and priorities (PRISMS); self-help guidebooks; access to community groups and programmes; and enhanced access to psychological therapists for IBS participants

Stages completed: Limited - B, C, E, F

Usual provider aware of patient's goals and action plans: Yes

Standardisation of clinician input: Weak - 2 training sessions + manual, but low levels

Personalised care planning for adults with chronic or long-term health conditions (Review) 
Kennedy 2013 (Continued)

of implementation

Fidelity: Poor - shared decision-making at 6 months significantly less in intervention than control group ( $\mathrm{P}=0.05)$; only $2 \%$ of IBS participants referred to therapists; $42 \%$ of clinicians failed to use PRISMS tool (p. 4). Process evaluation (Kennedy 2014) examined reasons for failure to change practice and confirmed that very little personalised care planning took place

Attrition: $19 \%$ at 6 months and $27 \%$ at 12 months lost to follow-up

Comparison: Usual care, including information and support

Outcomes

Health status: subjective: Medical Outcomes Study short form (SF-36), Euroqol (EQ5D)

Self-management capabilities: self efficacy*, patient enablement

Health behaviours: $n / a$

Achievement of personal goals: $n / a$

Service use: $\mathrm{n} / \mathrm{a}$

Adverse events: none reported

Length of follow-up: Baseline, 6 months, 12 months

*Primary outcomes. Power calculation - required sample of 40 practices and 48 participants per condition per practice (total participants $=5760$ ), so slightly under-powered

Risk of bias

Bias

Authors' judgement

Support for judgement

Random sequence generation (selection Low risk bias)

Wait-list comparator group. Used a minimisation procedure based on practice size, area deprivation and contractual status, practices were allocated 1:1 to intervention or control. Practices were paired as closely as possible according to their preferred training times, and using a minimisation procedure, 1 practice in each pair was allocated to training in the first year, with the other practice allocated to training at the same time the following year (p. 3)

Unclear risk

Research staff recruiting practices were unaware of the next allocation in the sequence at the time of recruitment (Bower 2012, p. 7). Baseline (and subsequent follow-up) data collection then took place at both practices in a pair at the same time. Proved impossible to recruit participants prior to allocation. Practices required adequate advance notice of their training date, hence it became necessary to inform them of their group allocation prior to participant selection. Authors confident that any resulting 
Kennedy 2013 (Continued)

bias is small. Recruitment was through electronic health records rather than by professional invitation, but practitioners could exclude patients after identification. These exclusions represented a relatively small proportion of patients (COPD 15\% intervention, $11 \%$ control; diabetes $11 \%$ int., $10 \%$ cont; IBS $11 \%$ int., $18 \%$ cont.)

Blinding of participants and personnel Unclear risk

Personnel were not blinded and outcomes (performance bias) were patients' self report

All outcomes

Blinding of outcome assessment (detection Low risk bias)

Analyst blind to practice allocation (supAll outcomes plementary file).

Incomplete outcome data (attrition bias) Low risk All outcomes

Intention-to-treat analysis. $81 \%$ completed 6 month follow-up and $72.8 \%$ the 12 month follow-up. Few differences between intervention and control in completeness of outcome data. Missing values for outcome variables at follow-up were not imputed, but addressed through covariate adjustment

Selective reporting (reporting bias) Low risk

Trial report matched published protocol apart from certain measures that were eventually omitted from the study to make the questionnaire shorter. No evidence of selective outcome reporting

Other bias

High risk

Fidelity to the intervention was very poor - shared decision making at 6 months significantly less in intervention than control group ( $\mathrm{P}=0.05)$; only $2 \%$ of IBS participants referred to therapists; $42 \%$ of clinicians failed to use PRISMS tool (p. 4). Kennedy 2014 confirms that very little personalised care planning actually took place, so we have excluded the study from the meta-analysis. No evidence of selective recruitment by clusters. Two trial arms were reasonably well-balanced on all variables at the participant level, but practices in the intervention group were on average slightly smaller (mean list size 4003 vs 4528 patients)

Personalised care planning for adults with chronic or long-term health conditions (Review)

Copyright $\Subset 2015$ The Cochrane Collaboration. Published by John Wiley \& Sons, Ltd. 


$\begin{array}{ll}\text { Participants } & \text { Country: China } \\ \text { Setting: Primary care } \\ \text { Conditions/numbers: } 208 \text { diabetes patients (type 2) (intervention 119, control 89) } \\ \text { Multi-morbidity: } n / a \\ \text { Health literacy: } n / a, 2 \text { rural communities. }\end{array}$

Type of intervention: Group visit + face-to-face consultation

Clinicians involved: Nurse (usual), GP (usual), preventive doctor (usual)

Tools: Participants were invited to attend a 12-session (monthly) group visit programme + 60-minute one-to-one visit with healthcare provider at the end of each group visit, if wanted (only $1 / 4$ received these). Programme followed Chronic Disease Self-Management Programme (CDSMP) format, including setting goals and making action plans. The format was adapted from the Chinese version of Stanford CDSMP Leaders Manual. The content included topics covered in the generic CDSMP course as well as diabetes specific self-management support topics recommended by Shanghai community diabetes prevention and control guidelines. Groups were led by existing general practice teams consisting of 1 GP, 1 preventive doctor, 1 nurse practitioner and 1 patient. Sessions focused on helping participants build confidence in their ability to deal with diabetes by incorporating self-efficacy-enhancing strategies, including action-planning and feedback, modelling of behaviours by participants for one another, reinterpretation of symptoms, practicing self-management skills, and group problem-solving. Participants made 121 week action plans over the 12 months. Each group also had a lay leader with diabetes who followed up with group members on their action plans in person or by telephone within 1 week. Staff attended a 1-day training workshop

Stages completed: Limited - B, C, F

Usual provider aware of patient's goals and action plans: Yes

Standardisation of clinician input: GPs and nurses involved in design of scripted programme implemented in 2 rural communities by 3 general practice teams +1 -day training workshop

Fidelity: $75.6 \%$ of participants attended 10 or more sessions. Patients who participated were significantly older with a higher prevalence of hypertension than those who declined Attrition: $15 \%$ lost to follow-up Comparison: Usual care provided by a single GP blood pressure (DBP); subjective: Self-rated health, energy, health distress, fatigue, illness intrusiveness, depression (Chinese adaptations of Stanford instruments)

Self-management capabilities: self efficacy, symptom management (Stanford)

Health behaviours: exercise (NV), diet (NV)

Achievement of personal goals: $n / a$

Service use: $n / a$

Adverse events: none reported 
Liu 2012 (Continued)

Length of follow-up: baseline, 12 months

\begin{tabular}{l|l|l}
\hline Notes & No primary outcome. No power calculation reported \\
\hline Risk of bias & Authors' judgement & Support for judgement \\
\hline Bias & Low risk & $\begin{array}{l}\text { Randomisation was by means of a random } \\
\text { number table with a ratio designed to yield } \\
\text { no fewer than 20 and no more than 25 } \\
\text { participants in a group. (p. 5) }\end{array}$ \\
\hline $\begin{array}{l}\text { Random sequence } \\
\text { bias }\end{array}$ & & (selection \\
\hline Allocation concealment (selection bias) & Unclear risk & Not reported. \\
\hline
\end{tabular}

Blinding of participants and personnel High risk (performance bias)

All outcomes

Participants were aware of their assignments. Both participants and personnel were aware of treatment status and a number of outcomes were subjective

Blinding of outcome assessment (detection Low risk bias)

All outcomes

Data collection was completed by university students who did not know the participants or their allocation status. All those assessing and analysing the data were blinded to group assignments (p. 5)

Incomplete outcome data (attrition bias) Low risk All outcomes

98 out of 119 in the intervention group completed the 12-month follow-up and 78 out of 89 in the control group. Reasons for loss to follow-up are documented in the flow diagram (p. 4)

Selective reporting (reporting bias) Unclear risk

No published protocol, but results presented for all listed outcome measures

Other bias

Unclear risk

No significant differences at baseline apart from prevalence of hypertension which was higher in the intervention group and fatigue and illness intrusiveness which were lower in the intervention group 
Methods
Study design: 4-arm pilot RCT, but only care management arm without group education included here

Unit of randomisation: patient

Unit of analysis: patient

Funding sources: National Institute of Mental Health

Conflicting interests: none declared

\begin{tabular}{ll}
\hline Participants & Country: USA \\
& Setting: Primary care \\
& Conditions/numbers: 52 patients with chronic or recurrent depression (26 care man- \\
agement intervention, 26 usual care) & Multi-morbidity: $\mathrm{n} / \mathrm{a}$ \\
Health literacy: $\mathrm{n} / \mathrm{a}$ \\
\hline
\end{tabular}

Interventions
Theoretical framework: Chronic Care Model

Focus: patient

Type of intervention: Telephone monitoring and care management + staff training Clinicians involved: Care manager (additional)

Tools: Telephone monitoring and care management - computerised decision support system supported systematic tracking of participant contacts, scripted clinical assessments, automatic application of treatment algorithms, and generation of feedback reports. The care manager (a master's level counsellor) contacted each participant at specified intervals (at least monthly during the first 3 months then at varying intervals) and helped them create a written care plan + education about medicines adherence and motivational enhancement. The care manager communicated with the treating provider when necessary. Care management training involved 4 hours of didactic training, 4 hours of role play, and direct observation of 2 care management contacts, followed by certification + weekly supervision

Stages completed: Extended - B, C, D, E, F

Usual provider aware of patient's goals and action plans: Yes

Standardisation of clinician input: Strong - single care manager, 4 hours didactic training +4 hours role play + direct observation of 2 contacts + certification + weekly supervision

Fidelity: not reported

Attrition: $18 \%$ lost to follow-up

Comparison: usual care
Health status: psychological: Structured Clinical Interview for DSM-IV (SCID) depression module, 20-item SCL depression scale, subjective: Patient-Rated Global Improvement (PGI)

Self-management capabilities: $\mathrm{n} / \mathrm{a}$

Health behaviours: $n / a$

Achievement of personal goals: $n / a$

Service use: $n / a$

Adverse events: none reported

Length of follow-up: baseline, 3, 6, 9 and 12 months
Author contacted and additional data supplied. Pilot study - no power calculation reported 
Ludman 2007 (Continued)

\section{Risk of bias}

\begin{tabular}{|c|c|c|}
\hline Bias & Authors' judgement & Support for judgement \\
\hline $\begin{array}{l}\text { Random sequence generation (selection } \\
\text { bias) }\end{array}$ & Low risk & $\begin{array}{l}\text { After baseline interview data manager as- } \\
\text { signed patients to } 1 \text { of } 4 \text { treatment groups } \\
\text { using computer-generated permuted block } \\
\text { design (p. 1066) }\end{array}$ \\
\hline Allocation concealment (selection bias) & Low risk & Concealed from interviewers. \\
\hline $\begin{array}{l}\text { Blinding of participants and personnel } \\
\text { (performance bias) } \\
\text { All outcomes }\end{array}$ & Unclear risk & Not possible to blind participants. \\
\hline $\begin{array}{l}\text { Blinding of outcome assessment (detection } \\
\text { bias) } \\
\text { All outcomes }\end{array}$ & Low risk & Interviewers and analysts were blinded. \\
\hline $\begin{array}{l}\text { Incomplete outcome data (attrition bias) } \\
\text { All outcomes }\end{array}$ & Low risk & $\begin{array}{l}\text { Usual care group participants completed } \\
92 \% \text { of all blinded follow-up interviews, } \\
\text { care management group completed } 82 \% \text {, } \\
\text { prof-led group completed } 94 \% \text { and peer- } \\
\text { led group completed } 83 \% \text { (p. } 1069 \text { ) }\end{array}$ \\
\hline Selective reporting (reporting bias) & Unclear risk & $\begin{array}{l}\text { No published protocol. All outcome mea- } \\
\text { sures mentioned, though not all in tabular } \\
\text { form. For example, detailed results for PGI } \\
\text { not reported in full and SCL only in graph- } \\
\text { ical form. Author contacted }\end{array}$ \\
\hline Other bias & Unclear risk & $\begin{array}{l}\text { There were some differences between } \\
\text { groups at baseline - authors do not report } \\
\text { on the significance of these }\end{array}$ \\
\hline
\end{tabular}

Naik 2011

\begin{tabular}{|c|c|}
\hline Methods & $\begin{array}{l}\text { Study design: RCT } \\
\text { Unit of randomisation: patient } \\
\text { Unit of analysis: patient } \\
\text { Funding sources: Agency for Healthcare Research and Quality; Doris Duke Charitable } \\
\text { Foundation; National Institute of Aging; Houston Health Services Research and Devel- } \\
\text { opment Center of Excellence } \\
\text { Conflicting interests: none declared }\end{array}$ \\
\hline Participants & $\begin{array}{l}\text { Country: USA } \\
\text { Setting: Primary care } \\
\text { Conditions/numbers: } 87 \text { diabetes patients ( } 45 \text { intervention, } 42 \text { control) }\end{array}$ \\
\hline
\end{tabular}


Naik 2011 (Continued)

\section{Multi-morbidity: n/a}

Health literacy: n/a

Interventions
Theoretical framework: $n / a$

Focus: patient

Type of intervention: Group visit + face-to-face consultation

Clinicians involved: Physicians (additional)

Tools: Empowering Patients in Care (EPIC) was a clinician-led, patient-centred group clinic consisting of 4 sessions on setting self-management action plans (diet, exercise, home monitoring, medications, etc.). This was followed by a 1-hour group session and a 10-min consultation with a clinician. Goals focused primarily on diet and exercise changes, home monitoring of blood glucose and medication effects, and communication with primary care providers about medications. The fourth session allowed for constructive reporting and feedback on participants' progress. Three primary care physicians led the sessions. Study clinicians sent a research note to participants' primary care physician after each session, consisting of participants diabetes $\mathrm{ABC}$ status, specific DM goals and action plans discussed and any changes made to medications. Action plans for nearly all participants included taking medications prescribed by primary care physicians and discussing subjective and objective effects of medications

Stages completed: Limited - B, C, F, G

Usual provider aware of patient's goals and action plans: Yes

Standardisation of clinician input: strong - 3 study physicians directed the group sessions

Fidelity: not reported

Attrition: 2 drop-outs (2.3\%), 12 (14\%) did not complete follow-up survey

Comparison: Traditional - 2 x 2-hour group education sessions with a diabetes educator and dietician followed by a visit with a primary care provider 12 weeks after enrolment

Outcomes

Health status: physical: blood glucose (HbA1c)

Self-management capabilities: diabetes self efficacy (Stanford)

Health behaviours: $n / a$

Achievement of personal goals: $\mathrm{n} / \mathrm{a}$

Service use: $\mathrm{n} / \mathrm{a}$

Adverse events: none reported

Length of follow-up: baseline, 3 months, 1 year

Notes

Power calculation - required 98 participants to detect moderate effect on $\mathrm{HbAlc}$, so slightly under-powered

\section{Risk of bias}

Bias

Random sequence generation (selection Unclear risk bias)
Authors' judgement

\section{Support for judgement}

After enrolment participants were randomised using a block randomisation of 10 (p. 454) 
Naik 2011 (Continued)

\begin{tabular}{|c|c|c|}
\hline Allocation concealment (selection bias) & Low risk & $\begin{array}{l}\text { Allocation of treatment group assignment } \\
\text { was blinded using sequentially numbered } \\
\text { and sealed envelopes }\end{array}$ \\
\hline $\begin{array}{l}\text { Blinding of participants and personnel } \\
\text { (performance bias) } \\
\text { All outcomes }\end{array}$ & Unclear risk & $\begin{array}{l}\text { Both participants and personnel were un- } \\
\text { blinded, but primary outcome was objec- } \\
\text { tive so not likely to affect risk of bias }\end{array}$ \\
\hline $\begin{array}{l}\text { Blinding of outcome assessment (detection } \\
\text { bias) } \\
\text { All outcomes }\end{array}$ & Low risk & $\begin{array}{l}\text { Primary outcome is objective so unlikely to } \\
\text { be affected by lack of blinding }\end{array}$ \\
\hline $\begin{array}{l}\text { Incomplete outcome data (attrition bias) } \\
\text { All outcomes }\end{array}$ & Low risk & $\begin{array}{l}43 \text { of } 45 \text { participants randomised to EPIC } \\
\text { attended some or all of the intervention } \\
\text { sessions, as did all } 42 \text { of participants ran- } \\
\text { domised to the traditional group. Only } 1 \\
\text { person from each group was lost to fol- } \\
\text { low-up and hence had no HbAlc outcome } \\
\text { measures. Diabetes Self-Efficacy Scale data } \\
\text { were available for } 75 \text { participants ( } 87 \%) \text { at } \\
\text { 1-year follow-up }\end{array}$ \\
\hline Selective reporting (reporting bias) & Unclear risk & $\begin{array}{l}\text { No published protocol, but both outcome } \\
\text { measures reported. }\end{array}$ \\
\hline Other bias & Low risk & \\
\hline
\end{tabular}

Schillinger 2009

Methods

Study design: 3-arm RCT (only automated telephone self-management support - ATSM and usual care included here)

Unit of randomisation: patient

Unit of analysis: patient

Funding sources: Commonwealth Fund; Agency for Healthcare Research and Quality; California Endowment; San Francisco Department of Public Health; California Healthcare Foundation; National Institutes of Health

Conflicting interests: none declared

Participants

Country: USA

Setting: Primary care

Conditions/numbers: 226 diabetes patients (type 2, poorly controlled) (112 ATDM intervention, 114 usual care)

Multi-morbidity: $n / a$

Health literacy: 59\% "limited" health literacy measured with the Test of Functional Health Literacy in Adults (ToFHLA). 42\% Spanish-speaking and 12\% Cantonese speakers 
Type of intervention: Structured self-management support (automated phone + nurse follow-up) vs. group visits (not included here)

Clinicians involved: Care manager (additional)

Tools: Improving Diabetes Efforts Across Language and Literacy (IDEALL) project. Automated Telephone Self-Management (ATSM) + follow-up calls from specially trained nurse care manager to promote collaborative goal setting in the form of behavioural 'action' plans. The ATSM is a pre-recorded automated telephone call that participants receive each week. Those answering 'out of range' receive a call back from a nurse care manager who helps participants problem-solve the issue identified in the report or any other concerns, with a focus on collaborative goal setting and action plans. The intervention also included individualised assessment, skills enhancement, health education, follow-up and support, access to community resources and continuity of clinical care. All care manager-participant interactions, including action plans created and achieved, are documented via a standardised record linked to the community health network record and shared with primary care physicians

Stages completed: Limited - B, C, F

Usual provider aware of patient's goals and action plans: Yes

Standardisation of clinician input: Strong (trained care managers)

Fidelity: 94\% completed at least 1 ATSM call,

Attrition: 10\% lost to follow-up

Comparison: Usual care

Health status: physical: HbA1c, systolic blood pressure (SBP), diastolic blood pressure (DBP), body mass index (BMI); subjective: Medical Outcomes Study short form (SF12)

Self-management capabilities: $\mathrm{n} / \mathrm{a}$

Health behaviours: diet, exercise. self monitoring of blood glucose, caring for feet, diabetes interference

Achievement of personal goals: self report

Service use: cost effectiveness

Adverse events: none reported

Length of follow-up: baseline, 12 months

No primary outcome. No power calculation reported.

Risk of bias

Bias

Random sequence generation (selection Unclear risk bias)
Authors' judgement

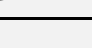


Schillinger 2009 (Continued)

\begin{tabular}{|c|c|c|}
\hline Allocation concealment (selection bias) & Unclear risk & Not reported. \\
\hline $\begin{array}{l}\text { Blinding of participants and personnel } \\
\text { (performance bias) } \\
\text { All outcomes }\end{array}$ & Unclear risk & $\begin{array}{l}\text { Blinding of participants and personnel not } \\
\text { possible, but most outcomes were objective }\end{array}$ \\
\hline $\begin{array}{l}\text { Blinding of outcome assessment (detection } \\
\text { bias) } \\
\text { All outcomes }\end{array}$ & Low risk & $\begin{array}{l}\text { Research assistants were masked to partici- } \\
\text { pants' group assignment (p. 565) }\end{array}$ \\
\hline $\begin{array}{l}\text { Incomplete outcome data (attrition bias) } \\
\text { All outcomes }\end{array}$ & Low risk & $\begin{array}{l}90 \% \text { completed follow-up interviews at } 1 \\
\text { year, HbA1c for } 88.2 \% \text {, BP for } 94.1 \% \text {, } \\
\text { BMI for } 92.3 \% \text {. Tested for difference be- } \\
\text { tween the } 2 \text { interventions due to attri- } \\
\text { tion bias - greater engagement was asso- } \\
\text { ciated with improvements in self-manage- } \\
\text { ment behaviour and functional status in } \\
\text { both arms, but did not alter size of effect } \\
\text { (p. } 564 \text { ) }\end{array}$ \\
\hline Selective reporting (reporting bias) & Unclear risk & No published protocol. \\
\hline Other bias & Low risk & \\
\hline
\end{tabular}

\section{Shearer 2007}

\begin{tabular}{ll}
\hline Methods & $\begin{array}{l}\text { Study design: RCT } \\
\text { Unit of randomisation: patient } \\
\text { Unit of analysis: patient } \\
\text { Funding sources: not stated } \\
\text { Conflicting interests: not stated }\end{array}$ \\
\hline Participants & $\begin{array}{l}\text { Country: USA } \\
\text { Setting: Secondary care } \\
\text { Conditions/numbers: } 90 \text { heart failure patients (45 intervention, } 45 \text { control) } \\
\text { Multi-morbidity: } n / a\end{array}$ \\
Health literacy: $n / a$
\end{tabular}


identify problems, goals and support needs. After sharing concerns and potential solutions, standardised questions related to weight, swelling in legs and abdomen, shortness of breath, chest pain and course of action if they experienced any of these symptoms ensued. The conversation remained open to the participant's needs and concerns, with the nurse providing support, encouragement, and information. Each telephone call was audiotaped to monitor intervention integrity. A summary of content discussed during each telephone call was documented in the participant's electronic medical record

Stages completed: Limited - B, C, F

Usual provider aware of patient's goals and action plans: Not stated

Standardisation of clinician input: strong ( 3 trained nurses following standard script, calls monitored)

Fidelity: good (participants received 6 phone calls in 12 weeks following discharge)

Attrition: low - 3\% lost to follow-up

Comparison: Usual care

Health status: subjective: Medical Outcomes Study short form (SF-36) mental and physical component scores (MCS/PCS)

Self-management capabilities: Power as Knowing Participation in Change Tool VII (PKPCT)*; Self Management of Heart Failure scale

Health behaviours: $n / a$

Achievement of personal goals: $n / a$

Service use: $\mathrm{n} / \mathrm{a}$

Adverse events: none reported

Length of follow-up: baseline, 12 weeks

\section{Risk of bias}

\begin{tabular}{l|ll}
\hline Bias & Authors' judgement & Support for judgement \\
\hline $\begin{array}{l}\text { Random sequence generation (selection } \\
\text { bias) }\end{array}$ & Unclear risk & No details given. \\
\hline $\begin{array}{l}\text { Allocation concealment (selection bias) } \\
\text { Blinding of participants and personnel } \\
\text { (performance bias) } \\
\text { All outcomes }\end{array}$ & High risk & No details given. \\
\hline $\begin{array}{l}\text { Blinding of outcome assessment (detection } \\
\text { bias) } \\
\text { All outcomes }\end{array}$ & Unclear risk & Both participants and personnel were un- \\
\hline $\begin{array}{l}\text { Incomplete outcome data (attrition bias) } \\
\text { All outcomes }\end{array}$ & High risk & blinded and outcomes were subjective \\
\hline
\end{tabular}


Shearer 2007 (Continued)

tively, at post-test. When $25 \%$ or fewer responses were found missing within a scale, the participant's scale mean was computed and substituted for a missing value; if less than $75 \%$ of the items on a scale were valid, the scale score was treated as missing and the case was excluded from the analysis of that specific outcome

\begin{tabular}{|c|c|c|}
\hline Selective reporting (reporting bias) & Unclear risk & No published protocol. \\
\hline Other bias & Unclear risk & $\begin{array}{l}\text { PKPCT has not been used with heart fail- } \\
\text { ure patients before and some participants } \\
\text { struggled to understand it }\end{array}$ \\
\hline
\end{tabular}

Stanhope 2013

\begin{tabular}{|c|c|}
\hline Methods & $\begin{array}{l}\text { Study design: cluster-RCT } \\
\text { Unit of randomisation: community mental health centre } \\
\text { Unit of analysis: patient } \\
\text { Funding sources: Janssen } \\
\text { Conflicting interests: fees/grants from Ortho-McNeil-Janssen and Forest Research In- } \\
\text { stitute }\end{array}$ \\
\hline Participants & $\begin{array}{l}\text { Country: USA } \\
\text { Setting: Community mental health centres, } 5 / 10 \text { randomised to training in person- } \\
\text { centred planning and collaborative documentation } \\
\text { Conditions/numbers: } 367 \text { mental health clients }(177 \text { intervention, } 190 \text { control): } \\
\text { schizophrenia }(n=153 \text {, bipolar disorder }(n=88) \text {, depression }(n=86) \text {, other }(n=40) \\
\text { Multi-morbidity: } n / a \\
\text { Health literacy: } n / a\end{array}$ \\
\hline
\end{tabular}

Interventions

Theoretical framework: $n / a$

Focus: both clinician and patient

Type of intervention: Staff training

Clinicians involved: Mental health providers (usual)

Tools: Clinicians received training in person-centred planning via video conferencing, followed by further coaching and monitoring during monthly meetings. Person-centred planning provides a blueprint to identify life goals that can be translated into action steps to inform the collaboration between the provider and the client. The process consisted of identifying life goals, assessing behavioural health problems, developing service plans to integrate life goals and behavioural health goals, and keeping a focus on life goals during the therapeutic sessions. Providers are also trained to focus on client engagement, following up at the next appointment to discuss missed appointments and problem-solve how to avoid them. Collaborative documentation consists of re-orienting assessment, planning, and evaluation documentation to identify and integrate personal goals with more traditional mental health goals and completing all documentation during face-toface sessions with the client

Personalised care planning for adults with chronic or long-term health conditions (Review) 
Stages completed: Limited - B, C, D, F

Usual provider aware of patient's goals and action plans: Yes

Standardisation of clinician input: Strong (training + coaching and monthly monitoring)

Fidelity: not reported

Attrition: not reported

Comparison: Usual care + centralised scheduling and management of no-shows

Health status: $\mathrm{n} / \mathrm{a}$

Self-management capabilities: $n / a$

Health behaviours: medication adherence

Achievement of personal goals: $n / a$

Service use: $\mathrm{n} / \mathrm{a}$

Adverse events: none reported

Length of follow-up: monthly for 11 months

\section{Risk of bias}

\begin{tabular}{|c|c|c|}
\hline Bias & Authors' judgement & Support for judgement \\
\hline $\begin{array}{l}\text { Random sequence generation (selection } \\
\text { bias) }\end{array}$ & Unclear risk & $\begin{array}{l}\text { Cluster-randomised trial, } 10 \mathrm{CMHCs,} 5 \\
\text { randomly allocated to intervention - no } \\
\text { further details provided }\end{array}$ \\
\hline Allocation concealment (selection bias) & Unclear risk & $\begin{array}{l}\text { No details provided, but concurrent inter- } \\
\text { vention with both groups may have helped } \\
\text { to conceal allocation }\end{array}$ \\
\hline
\end{tabular}

Blinding of participants and personnel Unclear risk Not possible to blind participants.

(performance bias)

All outcomes

Blinding of outcome assessment (detection High risk bias)

All outcomes
Incomplete outcome data (attrition bias) Unclear risk All outcomes

Selective reporting (reporting bias)

Unclear risk
Study relied on clinicians' reports of medication adherence and no-shows

\begin{tabular}{ll}
\hline Other bias $\quad$ Unclear risk
\end{tabular}
ratios for medication compliance over time (11 months)

No published protocol. Each of the main outcomes is reported

Insufficient baseline data to determine whether selective recruitment by cluster occurred or not. Client-level analyses were conducted separately for CMHCs in the 
Stanhope 2013 (Continued)

experimental and control groups to examine whether the odds of medication adherence changed over time. Given that the data included a monthly binary adherence measure for each client, random-effects logistic models were used to examine adherence (dependent variable) as a function of month (independent variable), including random effects for CMHCs and participants nested within CMHCs. The effect of time across the intervention groups was compared by including an intervention-bytime interaction term in a model containing both experimental and control sites. The results of the models were stratified by relevant participant and CMHC characteristics, and a 3-way interaction between the characteristic, the intervention, and time was calculated to determine whether any of these key factors moderated medication adherence. Logisitic regression models, including a random effect for site, were run to calculate the effect of the intervention on the odds of an appointment no-show. The models used data received from each $\mathrm{CMHC}$ on the total number of appointment no-shows and the total number of appointments (pp. 77-8). Clinician-recorded adherence was compared with participants' own reports and corroborated these (p. 79)

Thom 2013

\begin{tabular}{ll}
\hline Methods & Study design: RCT \\
& Unit of randomisation: patient \\
& Unit of analysis: patient \\
& Funding sources: American Academy of Family Physicians \\
Conflicting interests: not stated
\end{tabular}

Participants

Country: USA

Setting: Primary care

Conditions/numbers: 299 diabetes patients (148 intervention, 151 control)

Multi-morbidity: $\mathrm{n} / \mathrm{a}$

Health literacy: 36\% less than high school education, $46 \%$ primary language not English, 61\% income below USD 10,000

Interventions

Theoretical framework: $n / a$

Focus: patient

Type of intervention: training for peer coaches

Personalised care planning for adults with chronic or long-term health conditions (Review)

Copyright (c) 2015 The Cochrane Collaboration. Published by John Wiley \& Sons, Ltd. 
Thom 2013 (Continued)

Clinicians involved: peer coaches (additional)

Tools: Potential peer coaches attended 36 hours of training over 8 weeks in either English or Spanish. They were trained in active listening and non-judgemental communication, helping with diabetes self-management skills, providing social and emotional support, assisting with lifestyle change, facilitating medication understanding and adherence, navigating the clinic, and accessing community resources. Trainees who passed both a written and an oral examination became peer coaches in the study. Peer coaches interacted in person with the participants they coached at the discretion of the coach and participant, either outside the clinica by telephone or during a clinic visit. Target goals for coaching sessions were telephone contact at least twice a month and 2 or more in-person contacts over 6 months. Coaches helped participants design action plans to achieve goals chosen by the participant

Stages completed: Limited - B, C, F

Usual provider aware of patient's goals and action plans: Not stated

Standardisation of clinician input: Strong - peer coaches had 36 hours training over 8 weeks + written and oral examination

Fidelity: not reported

Attrition: 8\% dropped out

Comparison: Usual care included access to a nutritionist and diabetes educator through referral from primary care clinician

Outcomes

Health status: physical: blood glucose (HbA1c)*, cholesterol (LDL-C), systolic blood pressure (SBP), body mass index (BMI)

Self-management capabilities: $n / a$

Health behaviours: n/a

Achievement of personal goals: $n / a$

Service use: $\mathrm{n} / \mathrm{a}$

Adverse events: none reported

Length of follow-up: baseline, 6 months

Notes

*Primary outcome. Power calculation - 400 participants required to detect clinically significant difference in $\mathrm{HbAlc}$, so under-powered

Risk of bias

\begin{tabular}{lll}
\hline Bias & Authors judgement & Support for judgement \\
\hline $\begin{array}{l}\text { Random sequence generation (selection } \\
\text { bias) }\end{array}$ & Low risk & $\begin{array}{l}\text { Patients who enrolled and completed base- } \\
\text { line data collection were paid USD 10 and } \\
\text { assigned to the usual care or peer-coaching } \\
\text { arm using randomly ordered opaque en- } \\
\text { velopes (p. 139) }\end{array}$ \\
\hline Allocation concealment (selection bias) & Low risk & Opaque envelopes. \\
\hline
\end{tabular}

Blinding of participants and personnel Unclear risk

Not possible to blind participants.

(performance bias)

All outcomes

Personalised care planning for adults with chronic or long-term health conditions (Review)

Copyright $\Subset 2015$ The Cochrane Collaboration. Published by John Wiley \& Sons, Ltd. 
Thom 2013 (Continued)

Blinding of outcome assessment (detection Unclear risk bias)

All outcomes

Incomplete outcome data (attrition bias) Low risk All outcomes
No information about blinding of assessors.

24 (8\%) participants did not complete 6month data and were considered to have dropped out. These participants were likely to be younger, more likely to smoke, less likely to report having hyperlipidaemia, but otherwise did not vary significantly from remaining participants (p. 141)

Selective reporting (reporting bias) Unclear risk

No published protocol. All outcomes, attritions and exclusions reported, missing data treated as missing, not imputed

Other bias

Low risk

Tsay 2004

\begin{tabular}{|c|c|}
\hline Methods & $\begin{array}{l}\text { Study design: RCT } \\
\text { Unit of randomisation: patient } \\
\text { Unit of analysis: patient } \\
\text { Funding sources: National Science Council of Taiwan } \\
\text { Conflicting interests: not stated }\end{array}$ \\
\hline Participants & $\begin{array}{l}\text { Country: Taiwan } \\
\text { Setting: Dialysis centres in } 2 \text { hospitals } \\
\text { Conditions/numbers: } 50 \text { patients with end-stage renal disease (ESRD) }(25 \text { intervention, } \\
25 \text { control) } \\
\text { Multi-morbidity: } \mathrm{n} / \mathrm{a} \\
\text { Health literacy: } \mathrm{n} / \mathrm{a}\end{array}$ \\
\hline Interventions & $\begin{array}{l}\text { Theoretical framework: n/a } \\
\text { Focus: patient } \\
\text { Type of intervention: Structured, face-to-face self-management support } \\
\text { Clinicians involved: Nurse (additional) } \\
\text { Tools: The programme focused on helping participants develop skills and self awareness } \\
\text { in goal-setting, problem-solving, stress management, coping, social support and moti- } \\
\text { vation. It included participant identification of problem areas for self management of } \\
\text { ESRD, the exploration of emotions associated with these problems, the development } \\
\text { of a set of goals and strategies to overcome these problems and for achieving the goals, } \\
\text { making a behavioural change plan, and initiating self-care behaviours and stress man- } \\
\text { agement. Participants received an information package + individual consulting sessions } \\
3 \text { times a week for } 4 \text { weeks (p. } 61 \text { ) } \\
\text { Stages completed: Limited - B, C, F } \\
\text { Usual provider aware of patient's goals and action plans: Not stated } \\
\text { Standardisation of clinician input: Strong - single clinical nurse specialist provided }\end{array}$ \\
\hline
\end{tabular}

Personalised care planning for adults with chronic or long-term health conditions (Review)

Copyright $\Subset 2015$ The Cochrane Collaboration. Published by John Wiley \& Sons, Ltd. 
coaching (p. 61)

Fidelity: not reported

Attrition: no drop-outs

Comparison: Information package + usual care

Health status: psychological: Beck Depression Inventory
Self-management capabilities: Empowerment scale, Strategies used by People to Pro-
mote Health (SUPPH)
Health behaviours: $\mathrm{n} / \mathrm{a}$
Achievement of personal goals: $\mathrm{n} / \mathrm{a}$
Service use: $\mathrm{n} / \mathrm{a}$
Adverse events: none reported
Length of follow-up: baseline, 6 weeks

Notes No primary outcome. Power calculation performed and number of participants reported as adequate but few details provided

\section{Risk of bias}

\begin{tabular}{|c|c|c|}
\hline Bias & Authors' judgement & Support for judgement \\
\hline $\begin{array}{l}\text { Random sequence generation (selection } \\
\text { bias) }\end{array}$ & Low risk & $\begin{array}{l}\text { Participants were assigned to experimenta } \\
\text { or control group based on SPSS statistica } \\
\text { randomisation software (p. } 60 \text { ) }\end{array}$ \\
\hline
\end{tabular}

Allocation concealment (selection bias) Unclear risk

Researcher and nurse were aware of which treatments participants were receiving, but data collector was not

Blinding of participants and personnel Unclear risk (performance bias)

Participants' usual caregivers (physicians, All outcomes nurses, dieticians, and/or social workers) were uninformed about treatment group (p. 61), but not possible to blind participants

Blinding of outcome assessment (detection Low risk bias)

All outcomes

The data collector was a trained research assistant who was unaware of the participant's status to maintain double-blind accuracy. (p. 61)

Incomplete outcome data (attrition bias) Low risk

No drop-outs in either group.

All outcomes

Selective reporting (reporting bias)

Unclear risk

No published protocol. Results reported

for all outcome measures

Other bias

Low risk 


\begin{tabular}{ll}
\hline Methods & Study design: RCT \\
& Unit of randomisation: patient \\
& Unit of analysis: patient \\
& Funding sources: Dutch Diabetes Research Foundation \\
& Conflicting interests: none declared \\
\hline Participants & Country: Netherlands \\
& $\begin{array}{l}\text { Setting: Primary care } \\
\text { Conditions/numbers: } 119 \text { diabetes patients (type } 2)(59 \text { intervention, } 60 \text { control) } \\
\text { Multi-morbidity: } \mathrm{n} / \mathrm{a} \\
\text { Health literacy: } \mathrm{n} / \mathrm{a}\end{array}$ \\
\hline
\end{tabular}

Interventions
Theoretical framework: Bandura's Social Cognitive Theory

Focus: peer coach

Type of intervention: Training for peer coaches

Clinicians involved: Peer coaches (additional)

Tools: A peer-led self-management programme was developed with input from patients, GPs and dieticians. The primary objective was to increase self efficacy in patients with Type 2 diabetes. Secondary objectives were to improve physical activity and dietary habits. Five expert patients with diabetes were recruited through advertisements. They received 3 training sessions, each lasting 31/2 hours. They learnt the basic principles of motivational interviewing (how to support self efficacy, coping with resistance, showing empathy, exploring discrepancies). A script was developed for use by expert patients (peer coaches) who carried out 3 monthly 1-hour home visits to discuss participant's priorities, goals and action plans, with subsequent follow-up calls. During the first visit, areas for lifestyle change were explored. In the second visit, participants discussed the feasibility of lifestyle changes and set goals to work on over the next month. Progress towards the goals was evaluated in the third visit. Home visits lasted 1 hour on average. Within 2 weeks after each visit the expert patients contacted their participants by phone to evaluate the previous visit and answer any questions. Between visits participants could contact their expert patient by phone or email as often as they liked

Stages completed: Limited - B, C, F

Usual provider aware of patient's goals and action plans: not stated

Standardisation of clinician input: Strong - 5 expert patients received 3 x 31/2-hour training sessions in motivational interviewing + follow-up meetings and supervision ( $\mathrm{p}$. 391)

Fidelity: not reported

Attrition: 13 participants dropped out (11\%) and 23 did not return questionnaires $(19 \%)$

Comparison: Usual care

Outcomes

Health status: psychological: Center for Epidemiologic Studies Depression Scale (CESD); subjective: WHO Well-Being Index; Problem Areas in Diabetes (PAID-2)

Self-management capabilities: Diabetes Self-Efficacy*, Diabetes Coping;

Health behaviours: Physical Activity Scale for the Elderly, Fatlist

Achievement of personal goals: $n / a$

Service use: $\mathrm{n} / \mathrm{a}$

Adverse events: none reported

Length of follow-up: baseline, 3 months, 6 months 
Van der Wulp 2012 (Continued)

\begin{tabular}{|c|c|c|}
\hline Notes & \multicolumn{2}{|c|}{$\begin{array}{l}\text { * Primary outcome measure. Power calculation - } 80 \text { participants required to demonstrate } \\
\text { difference between groups in relation to self efficacy }\end{array}$} \\
\hline \multicolumn{3}{|l|}{ Risk of bias } \\
\hline Bias & Authors' judgement & Support for judgement \\
\hline $\begin{array}{l}\text { Random sequence generation (selection } \\
\text { bias) }\end{array}$ & Low risk & $\begin{array}{l}\text { A computerised randomisation model allo- } \\
\text { cated participants to intervention or con- } \\
\text { trol (p. } 396)\end{array}$ \\
\hline Allocation concealment (selection bias) & Low risk & $\begin{array}{l}\text { Randomisation conducted by person not } \\
\text { familiar with study or researchers }\end{array}$ \\
\hline $\begin{array}{l}\text { Blinding of participants and personnel } \\
\text { (performance bias) } \\
\text { All outcomes }\end{array}$ & Unclear risk & $\begin{array}{l}\text { Not possible to blind participants or peer } \\
\text { coaches. }\end{array}$ \\
\hline $\begin{array}{l}\text { Blinding of outcome assessment (detection } \\
\text { bias) } \\
\text { All outcomes }\end{array}$ & Unclear risk & Nothing reported re blinding of assessors. \\
\hline $\begin{array}{l}\text { Incomplete outcome data (attrition bias) } \\
\text { All outcomes }\end{array}$ & Low risk & $\begin{array}{l}\text { Missing data patterns were analysed and re- } \\
\text { vealed that data were missing completely } \\
\text { at random, so missing values were imputed } \\
\text { by means of regression analysis. Attrition } \\
\text { accounted for in detail (p. 392) }\end{array}$ \\
\hline Selective reporting (reporting bias) & Unclear risk & $\begin{array}{l}\text { No published protocol. All outcome mea- } \\
\text { sures reported. }\end{array}$ \\
\hline Other bias & Low risk & $\begin{array}{l}\text { Intervention took place in participants' } \\
\text { homes with peer coaches. Contamination } \\
\text { unlikely }\end{array}$ \\
\hline
\end{tabular}

Wilson 2010

Methods

Study design: 3-arm RCT (only shared decision-making arm (SDM) and usual care included in review)

Unit of randomisation: patient

Unit of analysis: patient

Funding sources: National Institutes of Health

Conflicting interests: fees/grants from Asthmatix, GlaxoSmithKline, AstraZeneca, Merck, Sepracor, Schering Plough, Pfizer, Palo Alto Medical Foundation Research Institute, Novartis, Bohringer Ingelheim, Vanguard Health Care, Kaiser Permanante 
Wilson 2010 (Continued)

\begin{tabular}{ll}
\hline Participants & Country: USA \\
& Setting: Primary care \\
Conditions/numbers: 408 asthma patients (asthma poorly controlled at baseline) (204 \\
SDM intervention, 204 usual care) \\
Multi-morbidity: $\mathrm{n} / \mathrm{a}$ \\
Health literacy: $\mathrm{n} / \mathrm{a}$ \\
\hline
\end{tabular}

Interventions

Theoretical framework: $n / a$

Focus: both patient and clinician

Type of intervention: Information + shared decision-making + follow-up phone calls + staff training

Clinicians involved: Care managers (usual)

Tools: Better Outcomes of Asthma Treatment (BOAT). Scripts were provided for use by specially trained care managers, together with visual aids and worksheets for participants. These were based on a shared decision-making process, involving stage-setting, gathering information from the participant (symptoms, perceptions of control, medication use, alternative treatments, environmental triggers, participant's goals and preferences), providing information (current understanding of asthma, review information and comprehension), negotiation (summarising goals and preferences, discussing options, negotiating decisions), wrapping-up (prescribe, give action plan, teach inhaler technique, give asthma diary), and 3 follow-up phone calls. At the end of session 1 a written asthma management and action plan was created, and potential barriers to medication adherence were elicited and addressed using motivational interviewing techniques. Care managers documented each encounter in the participant's chart, shared this with clinicians and discussed their recommendations

Stages completed: Limited - A, B, C, F

Usual provider aware of patient's goals and action plans: yes

Standardisation of clinician input: Strong - training for care managers + scripts + supervised tape-recorded practice sessions with feedback + monthly conference calls + ongoing quality control $-10 \%$ of sessions audiotaped + participants' reports

Fidelity: Good. Adherence to protocol formally assessed as high (online supplement p. 16)

Attrition: 11\% intervention, 7\% usual care lost to follow-up

Comparison: usual care

Outcomes

Health status: physical: Asthma Therapy Assessment Questionnaire (ATAQ)*, lung function - FEV1; subjective: Juniper Mini Asthma Quality of Life questionnaire*

Self-management capabilities: $\mathrm{n} / \mathrm{a}$

Health behaviours: medication adherence

Achievement of personal goals: $\mathrm{n} / \mathrm{a}$

Service use: asthma health care utilisation*

Adverse events: none reported

Length of follow-up: baseline, 12 months

Notes

* Primary outcomes. Author contacted and supplied additional data. No power calculation reported

\section{Risk of bias}

Personalised care planning for adults with chronic or long-term health conditions (Review) 


\begin{tabular}{|c|c|c|}
\hline Bias & Authors' judgement & Support for judgement \\
\hline $\begin{array}{l}\text { Random sequence generation (selection } \\
\text { bias) }\end{array}$ & Low risk & $\begin{array}{l}\text { A computer-based adaptive randomisation } \\
\text { algorithm was used (p. 567) }\end{array}$ \\
\hline Allocation concealment (selection bias) & Low risk & $\begin{array}{l}\text { Computer randomisation ensured conceal- } \\
\text { ment from staff. Randomisation was im- } \\
\text { plemented by having a designated, non- } \\
\text { blinded research staff member at the site en- } \\
\text { ter the relevant participant descriptors into } \\
\text { the randomisation module on the BOAT } \\
\text { website, which immediately performed the } \\
\text { randomisation, stored the result, and re- } \\
\text { turned the participant's study assignment } \\
\text { for implementation of the experimental as- } \\
\text { signment as indicated. All other study per- } \\
\text { sonnel, with the exception of the care man- } \\
\text { agers, were blinded to participant's study } \\
\text { assignment (online suppl. p. 3) }\end{array}$ \\
\hline
\end{tabular}

Blinding of participants and personnel Unclear risk (performance bias)

All outcomes
There was no intent that the participant's physicians be fully blinded to intervention assignment, nor obviously could the care manager be blinded to both the participation and study assignments of other participants, but they were not informed about this

All study personnel apart from care managers were blinded.

bias)

All outcomes

Incomplete outcome data (attrition bias) Low risk All outcomes

Selective reporting (reporting bias)

Unclear risk

Low risk
Missing data were not imputed: baseline and follow-up analyses were restricted to those participants with complete data for the analytic model variables at both time points (numbers on p. 570)

No published protocol. All outcomes reported. 


Country: USA
Petting: Community
Conditions/numbers: 56 diabetes patients $(30$ intervention, 26 control)
Multi-morbidity: $\mathrm{n} / \mathrm{a}$
Health literacy: $\mathrm{n} / \mathrm{a}$

Interventions

Theoretical framework: $\mathrm{n} / \mathrm{a}$

Focus: patient

Type of intervention: Information + structured coaching (phone)

Clinicians involved: Health coaches (additional)

Tools: Integrative Health coaching. The intervention group received a binder of educational materials at the initial visit. An initial telephone call then offered participants 30minute coaching sessions by telephone ( 8 weekly calls, 4 bi-weekly calls and 1 final call a month later). In the initial call participants were asked what was important to them in terms of diabetes care, how well they were managing their health, and what challenges they faced. The Wheel of Health (taking medicines as prescribed, stress reduction and self care, exercise, communication and relationships, nutrition, personal development) was used to guide the discussion. Priorities and goals were those of the participants. Goals were broken down into small, realistic action steps. Participants could select any goal for coaching support. Each participant received USD 75

Stages completed: Limited - A, B, C, F

Usual provider aware of patient's goals and action plans: not stated

Standardisation of clinician input: Strong - 2 experienced health coaches

Fidelity: not reported

Attrition: 7 withdrawals (12.5\%), 3 coaching, 4 control

Comparison: usual care

Outcomes

Health status: physical: blood glucose (HbA1c); psychological: Perceived Stress Scale (PSS4), subjective: Medical Outcomes Study short form (SF-12)

Self-management capabilities: Patient Activation Measure (PAM-13), Appraisal of Diabetes Scale (illness perception), Interpersonal Support Evaluation List (ISEL-12) (perceived social support), Benefit-Finding Scale (perceived benefits of the condition)

Health behaviours: Adherence - ASK-20, Morisky Adherence Scale, exercise frequency Achievement of personal goals: $n / a$

Service use: $\mathrm{n} / \mathrm{a}$

Adverse events: none reported

Length of follow-up: baseline, 6 months

No primary outcome. No power calculation reported.

Risk of bias

Bias

Authors' judgement

Support for judgement

Personalised care planning for adults with chronic or long-term health conditions (Review)

Copyright $\odot 2015$ The Cochrane Collaboration. Published by John Wiley \& Sons, Ltd. 
Wolever 2010 (Continued)

\begin{tabular}{l|l|l}
\hline $\begin{array}{l}\text { Random sequence generation (selection } \\
\text { bias) }\end{array}$ & Unclear risk & No details given \\
\hline $\begin{array}{l}\text { Allocation concealment (selection bias) } \\
\text { Blinding of participants and personnel } \\
\text { (performance bias) } \\
\text { All outcomes }\end{array}$ & High risk & No details given \\
\hline
\end{tabular}

Blinding of outcome assessment (detection Unclear risk

bias)

All outcomes

Pre-assessments and post-assessments were administered by blinded study staff but Incomplete outcome data (attrition bias) Low risk most outcomes are self-reported

All outcomes

Loss to follow-up - $3 / 30(10 \%)$ in the intervention group and $4 / 26(15 \%)$ in the control group. Reasons for withdrawal reported

Selective reporting (reporting bias) Unclear risk

No published protocol, but results reported for all outcomes

Other bias $\quad$ Low risk

\section{Zoffmann 2006}

Methods

Study design: RCT

Unit of randomisation: patient

Unit of analysis: patient

Funding sources: Danish Health Insurance Foundation; Novo Nordisk; Ely Lilly; Research Initiative in Aarhus; Danish Nurses' Organization; Aarhus University Hospital

Conflicting interests: not stated

Participants

Country: Denmark

Setting: Hospital outpatients

Conditions/numbers: 61 diabetes patients (type 1) (36 intervention, 25 control)

Multi-morbidity: $\mathrm{n} / \mathrm{a}$

Health literacy: $n / a$

Interventions

Theoretical framework: Prochaska's Stages of Change

Focus: patient

Type of intervention: Group visit + structured face-to-face coaching + staff training Clinicians involved: Nurses (additional)

Tools: Guided Self-Determination (GSD) aimed at increasing patients' life skills. Participants received group training + semi-structured worksheets + follow-up appointments either individually or in a group. Participants were prompted to systematically explore and express their personal difficulties through words and drawings. Reflections are recorded on worksheets designed to increase patients' ability to express their views and prepare them for active participation in the care process. Groups of about 10 members met over 
8 weeks for 2-hour sessions. A researcher introduced the sessions and worked together with GSD-trained nurses as coaches in smaller groups, supporting and challenging participants to develop their problem-solving skills. Participants set their own goals for future diabetes care. Three central worksheets comprising person-specific knowledge and agreements on strategies for problem-solving were saved in a folder in the participant's medical record for follow-up at outpatient appointments. Appointments between nurse and participant during 1-year follow-up were arranged either individually or on a group basis according to participant's preferences

Stages completed: Extended - A, B, C, D, F

Usual provider aware of patient's goals and action plans: not stated

Standardisation of clinician input: Strong - 7 training lectures + supervision

Fidelity: participants' reports indicated that GSD-GT-initiated autonomy support had taken place as intended (p. 84)

Attrition: 11 drop-outs (18\%)

Comparison: usual care

Random sequence generation (selection Low risk bias) bias)

All outcomes

Personalised care planning for adults with chronic or long-term health conditions (Review)

Randomisation took place at the 2 diabetes clinics. Written assignments were placed in sealed opaque envelopes, numbered and stacked randomly (p. 80)

If 2 participants were closely acquainted they were assigned to the same group. Can see why they did this but it means allocation was not completely concealed and not completely random (p. 80)

No blinding of participants and personnel possible.

Not blinded. 
Zoffmann 2006 (Continued)

\begin{tabular}{|c|c|c|}
\hline $\begin{array}{l}\text { Incomplete outcome data (attrition bias) } \\
\text { All outcomes }\end{array}$ & Low risk & $\begin{array}{l}\text { Rate of attrition reported and all outcome } \\
\text { measures given. }\end{array}$ \\
\hline Selective reporting (reporting bias) & Unclear risk & $\begin{array}{l}\text { No published protocol. All outcome mea- } \\
\text { sures reported. }\end{array}$ \\
\hline Other bias & Low risk & \\
\hline
\end{tabular}

NV: not validated

Characteristics of excluded studies [ordered by study ID]

\begin{tabular}{|c|c|}
\hline Study & Reason for exclusion \\
\hline Alamo 2002 & Not collaborative - clinicians decide on care plan. \\
\hline Anderson 2005 & Patient education only. \\
\hline Bieber 2006 & Collaborative goal-setting element is insufficient. More akin to a decision aid trial \\
\hline Brown 2005 & Collaborative goal-setting element is insufficient, only self-management support \\
\hline Chambers 2008 & $\begin{array}{l}\text { Intervention involves decision counselling and problem-solving without collaborative action-plan- } \\
\text { ning and goal-setting }\end{array}$ \\
\hline Chin 2007 & There is no action-planning and the way participants are involved is unclear \\
\hline Coleman 2006 & $\begin{array}{l}\text { No collaboration. Patient information/education and personal health record only. Goal and plans } \\
\text { restricted to medicines only }\end{array}$ \\
\hline Cooper 2011 & $\begin{array}{l}\text { Intervention is aimed specifically at overcoming barriers without collaborative action-planning or } \\
\text { goal-setting }\end{array}$ \\
\hline Cooper 2013 & Participants are involved, but not in action-planning or goal-setting process \\
\hline Deen 2011 & Intervention lacks collaborative care planning. \\
\hline Druss 2010 & $\begin{array}{l}\text { Educational intervention where participants are taught to create a care plan, rather than making one } \\
\text { in collaboration }\end{array}$ \\
\hline Eakin 2007 & Goals predetermined and constrained - diet and exercise only \\
\hline Estabrooks 2005 & Initial goals are set using a computer programme, not in collaboration \\
\hline
\end{tabular}


(Continued)

\begin{tabular}{|c|c|}
\hline Glasgow 2010 & No real engagement between patient and professional. \\
\hline Halpern 2004 & Goals and actions planned restricted to medication or psychotherapy \\
\hline Hamann 2006 & Intervention lacks collaborative goal-setting and action-planning \\
\hline Harris 2009 & Intervention lacks collaborative goal-setting and action-planning \\
\hline Heisler 2013 & $\begin{array}{l}\text { Intervention lacks collaborative goal-setting and action-planning. Collaborative discussion with } \\
\text { nurse is optional }\end{array}$ \\
\hline Joosten 2011 & No action-planning and predetermined limited goals only. \\
\hline Kilbourne 2013 & Patient education only. \\
\hline Koelewijn-van Loon 2010 & Most participants do not have long-term conditions. \\
\hline Lin 2006 & Restricted options and most decisions made by nurse and other clinicians \\
\hline Maindal 2011 & Little evidence of collaborative planning. \\
\hline McKay 2002 & Care planning is limited to dietary changes only. \\
\hline Patja 2012 & Little evidence of collaborative planning. \\
\hline Redfern 2010 & Predetermined options and plans, Little opportunity for patients to influence \\
\hline Richardson 2010 & Little evidence of collaborative planning. \\
\hline Riley 2001 & Goals predetermined and constrained - exercise, diet, smoking only \\
\hline Ruggiero 2010 & Patient education only. Goals based on provider recommendations \\
\hline Ruland 2003 & Not collaborative. \\
\hline Sciamanna 2011 & Not collaborative. \\
\hline Simon 2002 & Little evidence of collaborative goal setting or planning. \\
\hline Simon 2011 & Little evidence of collaborative planning. \\
\hline Smeulders 2009 & Patient education only. \\
\hline Smith 2008 & Patient information only. \\
\hline Sobell 2000 & Collaboration between patient and spouse, not clinician. \\
\hline
\end{tabular}


(Continued)

\begin{tabular}{ll}
\hline Sol 2008 & Action plans developed by nurse, not collaboratively. \\
\hline Street 2010 & Focused on communication only, not action-planning/ \\
\hline Stringer 2011 & Goals determined by professional team. \\
\hline Van GestelTimmermans 2012 & Patient education only. \\
\hline Vestala 2013 & Participation in documentation only, no collaborative goal setting or action-planning \\
\hline Von Korff 2003 & Prescriptive. Very little collaboration involved. \\
\hline Walker 2005 & Information only. \\
\hline Wennberg 2010 & Not possible to isolate those patients with long-term conditions \\
\hline Woltmann 2011 & Very little collaboration involved - client and case manager complete electronic plans individually \\
\hline Wright 2003 & No evidence of collaborative goal setting or action-planning \\
\hline
\end{tabular}

Characteristics of ongoing studies [ordered by study ID]

\section{Altiner 2012}

\begin{tabular}{ll}
\hline Trial name or title & MultiCare AGENDA \\
\hline Methods & 2-arm cluster-RCT \\
\hline Participants & Patients aged 65-84 with at least 3 chronic conditions \\
\hline Interventions & $\begin{array}{l}\text { Clinician-focused. Training for GPs in planned, structured collaborative consultations and narrative-based } \\
\text { medicine }\end{array}$ \\
\hline Outcomes & EQ-5D, Health Care Empowerment questionnaire, medication use, Leipzig Supply and Cost \\
\hline Starting date & Not stated \\
\hline Contact information & in.schaefer@uke.de \\
\hline Notes & ISRCTN46272088 \\
\hline
\end{tabular}


Bachman-Mettler 2011

\begin{tabular}{ll} 
Trial name or title & Case management in oncology rehabilitation (CAMON) \\
\hline Methods & Multi-centre, 2-arm RCT \\
\hline Participants & Patients aged 18 and over with any type of cancer \\
\hline Interventions & $\begin{array}{l}\text { Clinician-focused. Training for case managers (rehabilitation coaches) on how to provide self-management } \\
\text { support, including goal-setting, action-planning and review }\end{array}$ \\
\hline Outcomes & $\begin{array}{l}\text { FACT-G quality of life, activity restrictions, Jerusalem \& Schwarzer questionnaire (self management and } \\
\text { perceived self efficacy), PACIC-5A }\end{array}$ \\
\hline Starting date & May 2010 \\
\hline Contact information & irene.bachmann@usz.ch \\
\hline Notes & ISRCTN41474586 \\
\hline
\end{tabular}

Battersby 2010

\begin{tabular}{|c|c|}
\hline Trial name or title & Flinders Program \\
\hline Methods & Practice-based RCT \\
\hline Participants & $\begin{array}{l}\text { Patients aged over } 45 \text { with COPD, CAD, cerebrovascular disease, chronic heart failure, diabetes, muscu- } \\
\text { loskeletal disorders }\end{array}$ \\
\hline Interventions & $\begin{array}{l}\text { Focused both on patients and clinicians. Set of tools to enable health workers and patients to collaboratively } \\
\text { identify problems, set goals, and develop individual care plans covering self care, medical, psychosocial and } \\
\text { carer issues }\end{array}$ \\
\hline Outcomes & SF-12, Partners in Health scale, Stanford measures \\
\hline Starting date & Sept 2009 \\
\hline Contact information & melanie.harris@flinders.edu.au \\
\hline Notes & \\
\hline
\end{tabular}

\section{Coventry 2012}

\begin{tabular}{ll}
\hline Trial name or title & Collaborative Interventions for Circulation and Depression (COINCIDE) \\
\hline Methods & Pragmatic cluster-RCT \\
\hline Participants & Patients aged 18 and over with diabetes and/or coronary heart disease plus depression
\end{tabular}




\section{Coventry 2012 (Continued)}

\begin{tabular}{ll}
\hline Interventions & $\begin{array}{l}\text { Clinician-focused. Training + supervision in collaborative goal-setting and action-planning for psychological } \\
\text { well-being practitioners }\end{array}$ \\
\hline Outcomes & $\begin{array}{l}\text { EQ-5D, WHOQoL-BREF, Diabetes Quality of Life, Seattle Angina Questionnaire, Generalized Anxiety } \\
\text { Disorder (GAD-7), Sheehan Disability Scale, Relationship Scales Questionnaire (RSQ), Stanford Self-Efficacy } \\
\text { scale, Health Education Impact Questionnaire (heiQ), PACIC-5A, ENRICHD Social Support Instrument } \\
(\text { ESSI), Patient Service Utilization Questionnaire }\end{array}$ \\
\hline Starting date & Not stated \\
\hline Contact information & peter.a.coventry@manchester.ac.uk \\
\hline Notes & ISRCTN80309252
\end{tabular}

Reed 2011

\begin{tabular}{ll}
\hline Trial name or title & Flinders Program (2) \\
\hline Methods & RCT \\
\hline Participants & Patients aged 60 and over with 2 or more chronic conditions \\
\hline Interventions & $\begin{array}{l}\text { Focused both on patients and clinicians. Set of tools to enable health workers and patients to collaboratively } \\
\text { identify problems, set goals, and develop individual care plans covering self-care, medical, psychosocial and } \\
\text { carer issues }\end{array}$ \\
\hline Outcomes & $\begin{array}{l}\text { Stanford scales for fatigue, pain, health distress, energy, illness intrusiveness, PHQ-9, self efficacy, heiQ, } \\
\text { Flinders scales,exercise, medication adherence, GP visits, ED visits, hospital admissions }\end{array}$ \\
\hline Starting date & Not stated \\
\hline Contact information & richard.reed@flinders.edu.au \\
\hline Notes & \\
\hline
\end{tabular}

Tylee 2012

\begin{tabular}{ll}
\hline Trial name or title & UPBEAT-UK \\
\hline Methods & Pilot RCT \\
\hline Participants & Patients aged 18 and over with CHD and depression \\
\hline Interventions & $\begin{array}{l}\text { Patient-focused. Case managers working with patients on a collaborative basis to develop a personalised care } \\
\text { plan }\end{array}$
\end{tabular}


Tylee 2012 (Continued)

\begin{tabular}{|c|c|}
\hline Outcomes & $\begin{array}{l}\text { HADS depression sub-scale, PHQ-9, Modified Rose Angina questionnaire, specific activity schedule, Guy's } \\
\text { hospital chest pain questionnaire, EQ-5D, SF-12, Warwick-Edinburgh Mental Well-Being scale, Brief Illness } \\
\text { Perceptions Questionnaire, Psychlops, adapted Morisky adherence questionnaire, Client Service Receipt In- } \\
\text { ventory (CSRI) }\end{array}$ \\
\hline Starting date & 2011 \\
\hline Contact information & a.tylee@iop.kcl.ac.uk \\
\hline Notes & ISRCTN21615909 \\
\hline \multicolumn{2}{|l|}{ Van der Voort 2011} \\
\hline Trial name or title & Collaborative Care \\
\hline Methods & 2-arm cluster-RCT \\
\hline Participants & Patients aged $18-65$ with bipolar disorder \\
\hline Interventions & $\begin{array}{l}\text { Focused both on patients and clinicians. Formulation of Collaborative Care team including patient and } \\
\text { family member or friend. All decisions to be shared; development of personalised care plan; psycho education; } \\
\text { problem-solving treatment; mood-charting }\end{array}$ \\
\hline Outcomes & $\begin{array}{l}\text { Functioning Assessment Short Test (FAST-NL-P), Clinical Global Impression for Bipolar Disorder (CGI- } \\
\text { BP), Brief Symptom Inventory, Quick Inventory for Depressive Symptoms (QIDS-SR), Altman Self Rating } \\
\text { Mania Scale, Life Chart Method, WHOQoL-BREF, Sense of Mastery scale, costs - TiC-P }\end{array}$ \\
\hline Starting date & Not stated \\
\hline Contact information & n.vandervoort@ggzingeest.nl \\
\hline Notes & \\
\hline
\end{tabular}


DATA AND ANALYSES

Comparison 1. Physical health (personalised care planning vs usual care)

\begin{tabular}{lccll} 
Outcome or subgroup title & $\begin{array}{c}\text { No. of } \\
\text { studies }\end{array}$ & $\begin{array}{c}\text { No. of } \\
\text { participants }\end{array}$ & Statistical method & Effect size \\
\hline 1 HbA1c (change) & 9 & 1916 & Mean Difference (IV, Fixed, 95\% CI) & $-0.24[-0.35,-0.14]$ \\
2 SBP (change) & 6 & 1200 & Mean Difference (IV, Fixed, 95\% CI) & $-2.64[-4.47,-0.82]$ \\
3 DBP (change) & 4 & 751 & Mean Difference (IV, Fixed, 95\% CI) & $-0.71[-2.26,0.84]$ \\
4 Cholesterol (change) & 5 & 1545 & Std. Mean Difference (IV, Fixed, 95\% CI) & $0.01[-0.09,0.11]$ \\
5 BMI (change) & 4 & 822 & Mean Difference (IV, Fixed, 95\% CI) & $-0.11[-0.35,0.13]$ \\
\hline
\end{tabular}

Comparison 2. Psychological health (personalised care planning vs usual care)

\begin{tabular}{lccccc} 
Outcome or subgroup title & $\begin{array}{c}\text { No. of } \\
\text { studies }\end{array}$ & $\begin{array}{c}\text { No. of } \\
\text { participants }\end{array}$ & Statistical method & Effect size \\
\hline 1 Depression & 5 & 599 & Std. Mean Difference (IV, Fixed, 95\% CI) & $-0.36[-0.52,-0.20]$ \\
\hline
\end{tabular}

Comparison 3. Subjective health status (personalised care planning vs usual care)

\begin{tabular}{lcclll} 
Outcome or subgroup title & $\begin{array}{c}\text { No. of } \\
\text { studies }\end{array}$ & $\begin{array}{c}\text { No. of } \\
\text { participants }\end{array}$ & Statistical method & Effect size \\
\hline 1 Generic health status (physical) & 3 & 345 & Std. Mean Difference (IV, Fixed, 95\% CI) & $0.16[-0.05,0.38]$ \\
2 Generic health status (mental) & 3 & 345 & Std. Mean Difference (IV, Fixed, 95\% CI) & $0.07[-0.15,0.28]$ \\
3 Condition-specific health status & 4 & 1330 & Std. Mean Difference (IV, Fixed, 95\% CI) & $-0.01[-0.11,0.10]$ \\
\hline
\end{tabular}

Comparison 4. Self-management capabilities (personalised care planning vs usual care)

\begin{tabular}{lccccc} 
Outcome or subgroup title & $\begin{array}{c}\text { No. of } \\
\text { studies }\end{array}$ & $\begin{array}{c}\text { No. of } \\
\text { participants }\end{array}$ & Statistical method & Effect size \\
\hline 1 Self efficacy & 5 & 471 & Std. Mean Difference (IV, Fixed, 95\% CI) & $0.25[0.07,0.43]$ \\
\hline
\end{tabular}


Comparison 5. Health-related behaviours (personalised care planning vs usual care)

\begin{tabular}{lccccc} 
Outcome or subgroup title & $\begin{array}{c}\text { No. of } \\
\text { studies }\end{array}$ & $\begin{array}{c}\text { No. of } \\
\text { participants }\end{array}$ & Statistical method & Effect size \\
\hline 1 Exercise & 6 & 907 & Std. Mean Difference (IV, Fixed, 95\% CI) & $0.11[-0.02,0.24]$ \\
\hline
\end{tabular}

Comparison 6. Self-care activities (personalised care planning vs usual care)

\begin{tabular}{lccccc} 
Outcome or subgroup title & $\begin{array}{c}\text { No. of } \\
\text { studies }\end{array}$ & $\begin{array}{c}\text { No. of } \\
\text { participants }\end{array}$ & Statistical method & Effect size \\
\hline 1 Self care (days per week) & 4 & 520 & Std. Mean Difference (IV, Fixed, 95\% CI) & $0.35[0.17,0.52]$ \\
\hline
\end{tabular}

Comparison 7. Type of intervention (HbA1c) (extended vs limited)

\begin{tabular}{ccccc} 
Outcome or subgroup title & $\begin{array}{c}\text { No. of } \\
\text { studies }\end{array}$ & $\begin{array}{c}\text { No. of } \\
\text { participants }\end{array}$ & Statistical method & Effect size \\
\hline 1 HbA1c (change) & 9 & 1916 & Mean Difference (IV, Fixed, 95\% CI) & $-0.24[-0.35,-0.14]$ \\
1.1 Extended & 3 & 408 & Mean Difference (IV, Fixed, 95\% CI) & $-0.43[-0.60,-0.26]$ \\
1.2 Limited & 6 & 1508 & Mean Difference (IV, Fixed, 95\% CI) & $-0.12[-0.26,0.02]$ \\
\hline
\end{tabular}

Comparison 8. Type of intervention (HbA1c) (high intensity vs low intensity)

\begin{tabular}{lcclr} 
Outcome or subgroup title & $\begin{array}{c}\text { No. of } \\
\text { studies }\end{array}$ & $\begin{array}{c}\text { No. of } \\
\text { participants }\end{array}$ & Statistical method & Effect size \\
\hline 1 HbA1c (change) & 9 & 1916 & Mean Difference (IV, Fixed, 95\% CI) & $-0.24[-0.35,-0.14]$ \\
1.1 High & 5 & 847 & Mean Difference (IV, Fixed, 95\% CI) & $-0.43[-0.63,-0.24]$ \\
1.2 Low & 4 & 1069 & Mean Difference (IV, Fixed, 95\% CI) & $-0.17[-0.29,-0.04]$ \\
\hline
\end{tabular}




\begin{tabular}{ccccr} 
Outcome or subgroup title & $\begin{array}{c}\text { No. of } \\
\text { studies }\end{array}$ & $\begin{array}{c}\text { No. of } \\
\text { participants }\end{array}$ & Statistical method & Effect size \\
\hline 1 HbA1c (change) & 9 & 1916 & Mean Difference (IV, Fixed, 95\% CI) & $-0.24[-0.35,-0.14]$ \\
1.1 High & 2 & 358 & Mean Difference (IV, Fixed, 95\% CI) & $-0.45[-0.70,-0.21]$ \\
1.2 Low & 7 & 1558 & Mean Difference (IV, Fixed, 95\% CI) & $-0.19[-0.31,-0.08]$ \\
\hline
\end{tabular}

\section{Analysis I.I. Comparison I Physical health (personalised care planning vs usual care), Outcome I HbAlc (change).}

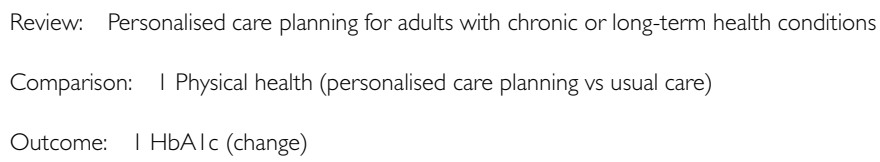

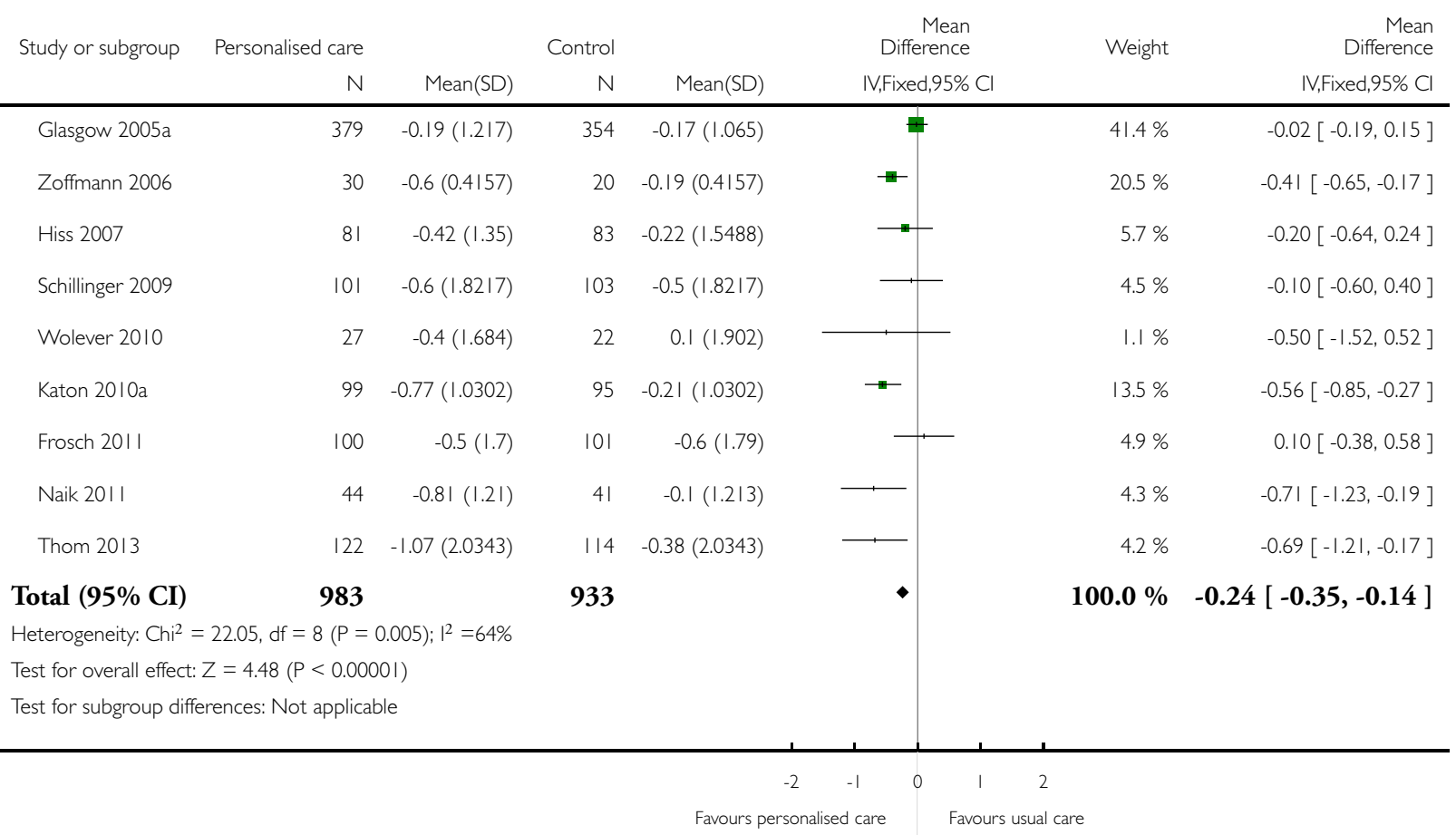


Analysis I.2. Comparison I Physical health (personalised care planning vs usual care), Outcome 2 SBP (change).

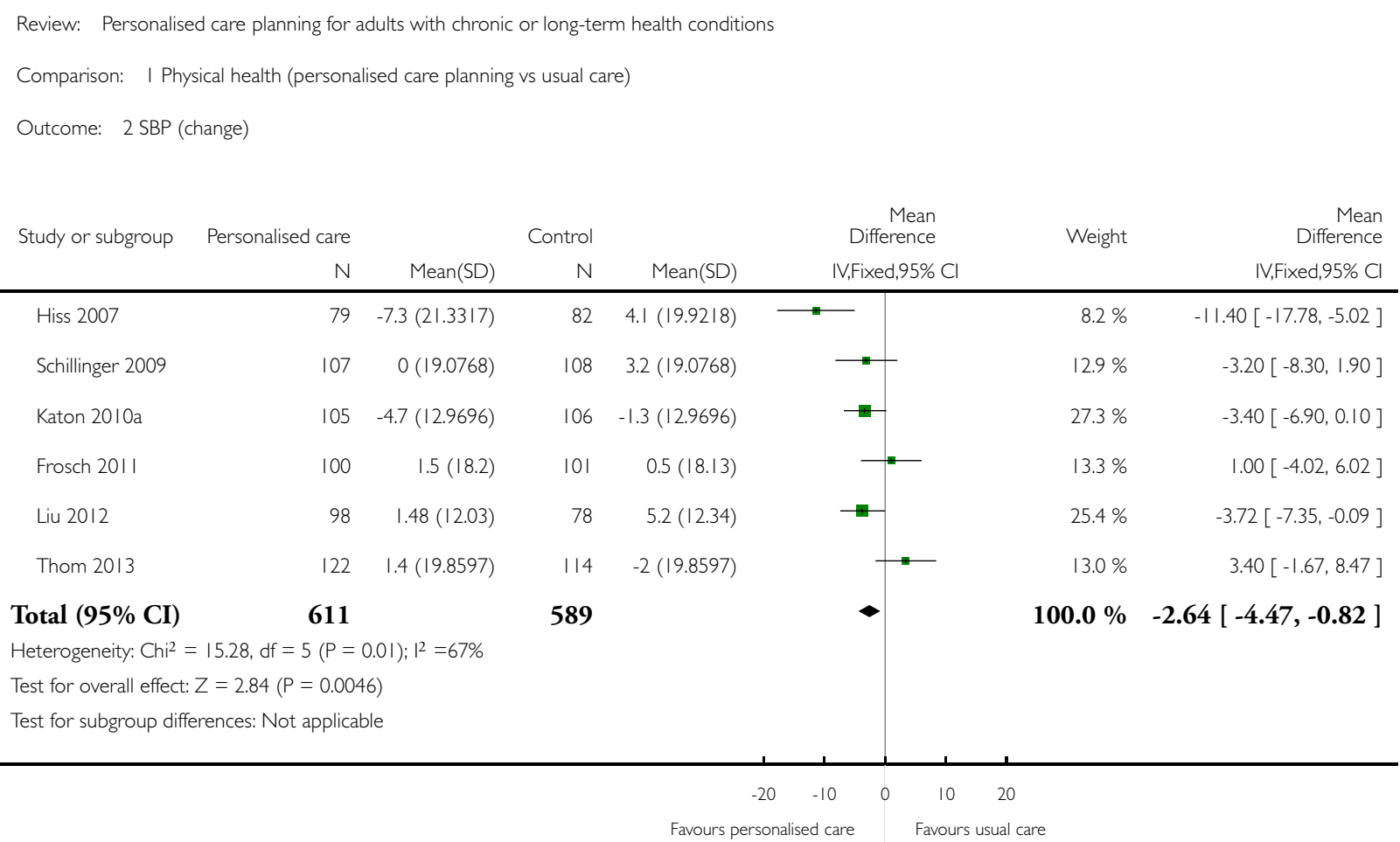


Analysis I.3. Comparison I Physical health (personalised care planning vs usual care), Outcome 3 DBP (change).

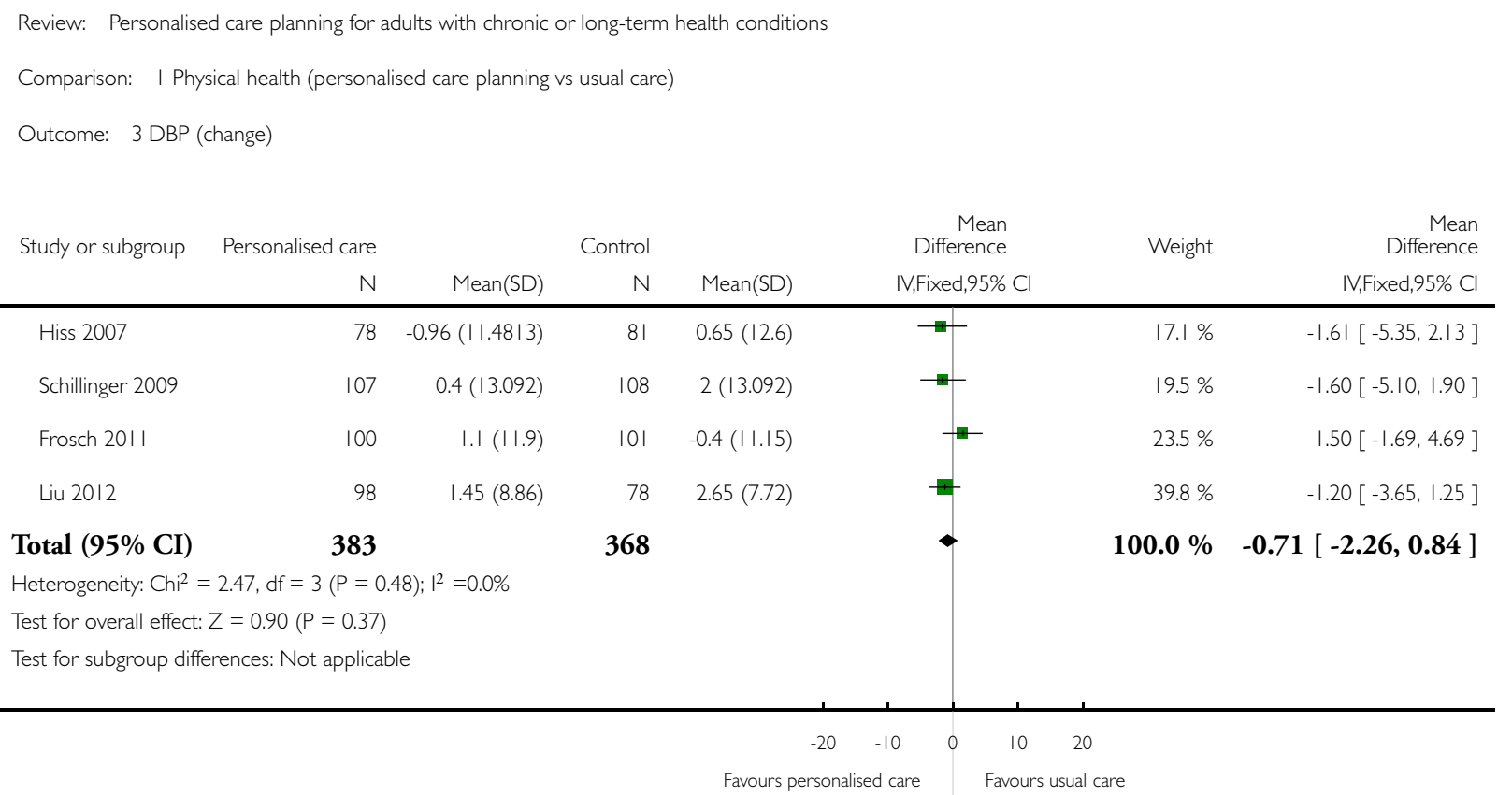


Analysis I.4. Comparison I Physical health (personalised care planning vs usual care), Outcome 4 Cholesterol (change).

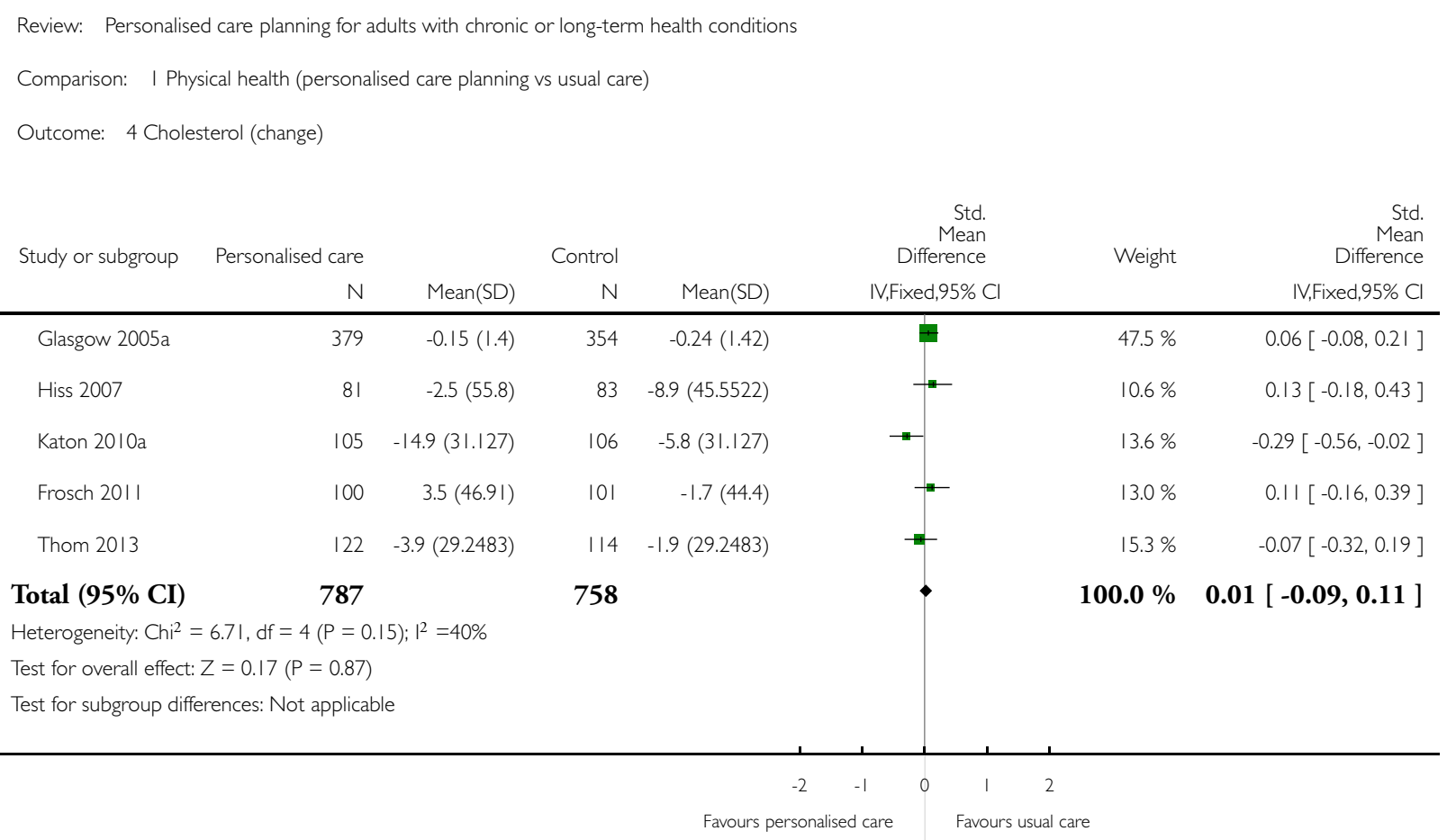


Analysis I.5. Comparison I Physical health (personalised care planning vs usual care), Outcome 5 BMI (change).

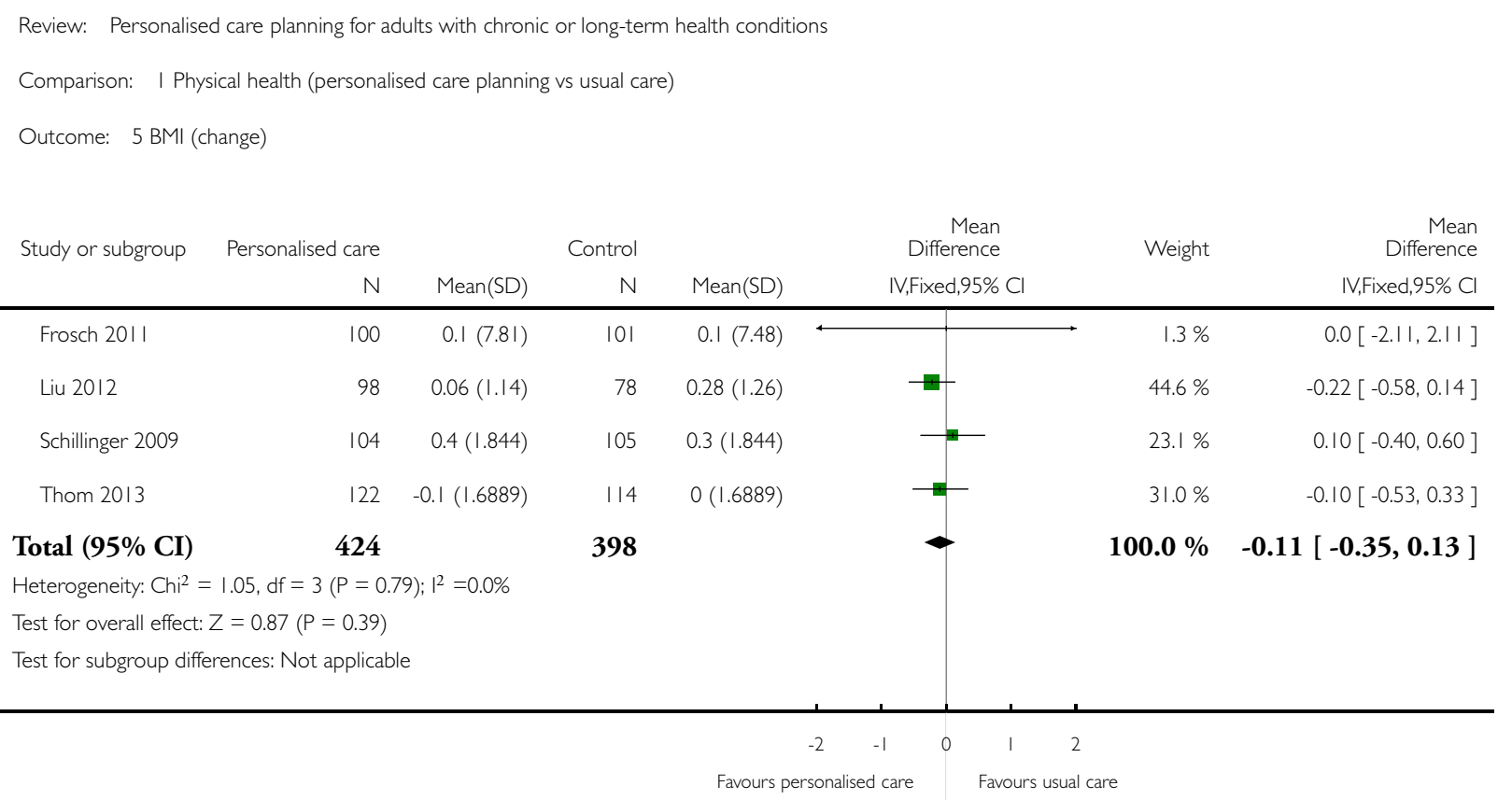


Analysis 2.I. Comparison 2 Psychological health (personalised care planning vs usual care), Outcome I Depression.

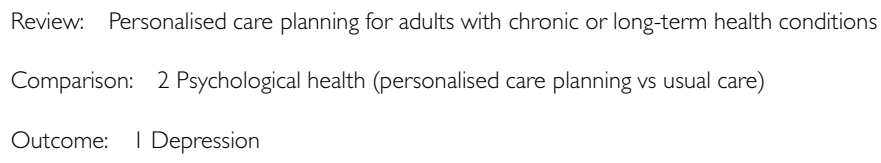

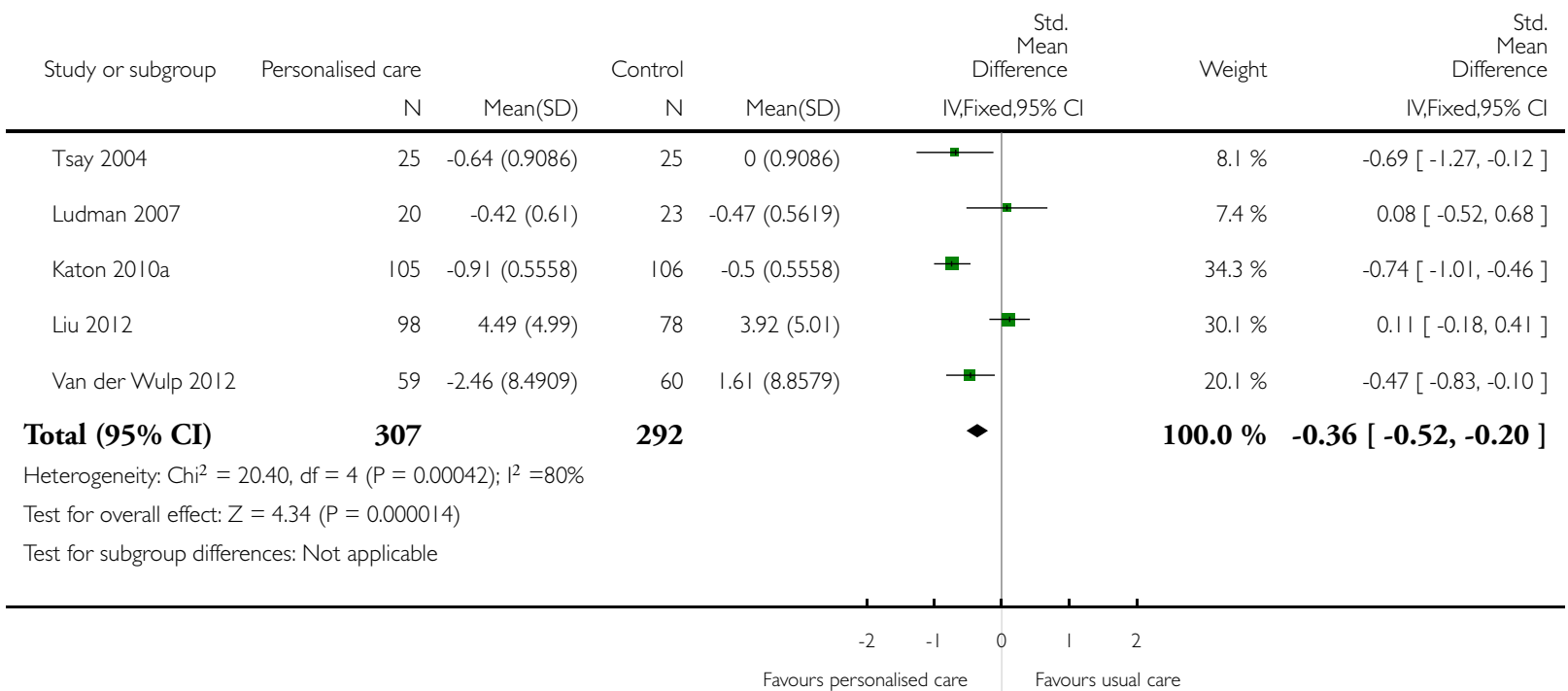


Analysis 3.I. Comparison 3 Subjective health status (personalised care planning vs usual care), Outcome I Generic health status (physical).

Review: Personalised care planning for adults with chronic or long-term health conditions

Comparison: 3 Subjective health status (personalised care planning vs usual care)

Outcome: I Generic health status (physical)

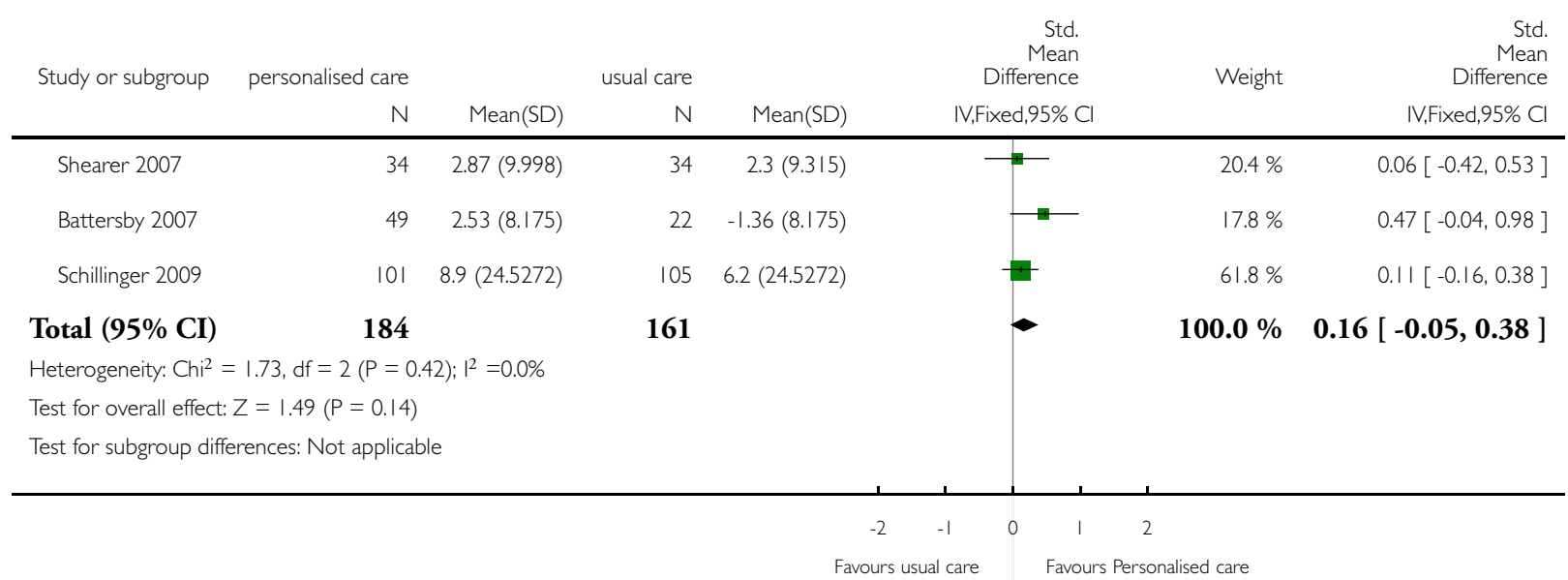


Analysis 3.2. Comparison 3 Subjective health status (personalised care planning vs usual care), Outcome 2 Generic health status (mental).

Review: Personalised care planning for adults with chronic or long-term health conditions

Comparison: 3 Subjective health status (personalised care planning vs usual care)

Outcome: 2 Generic health status (mental)

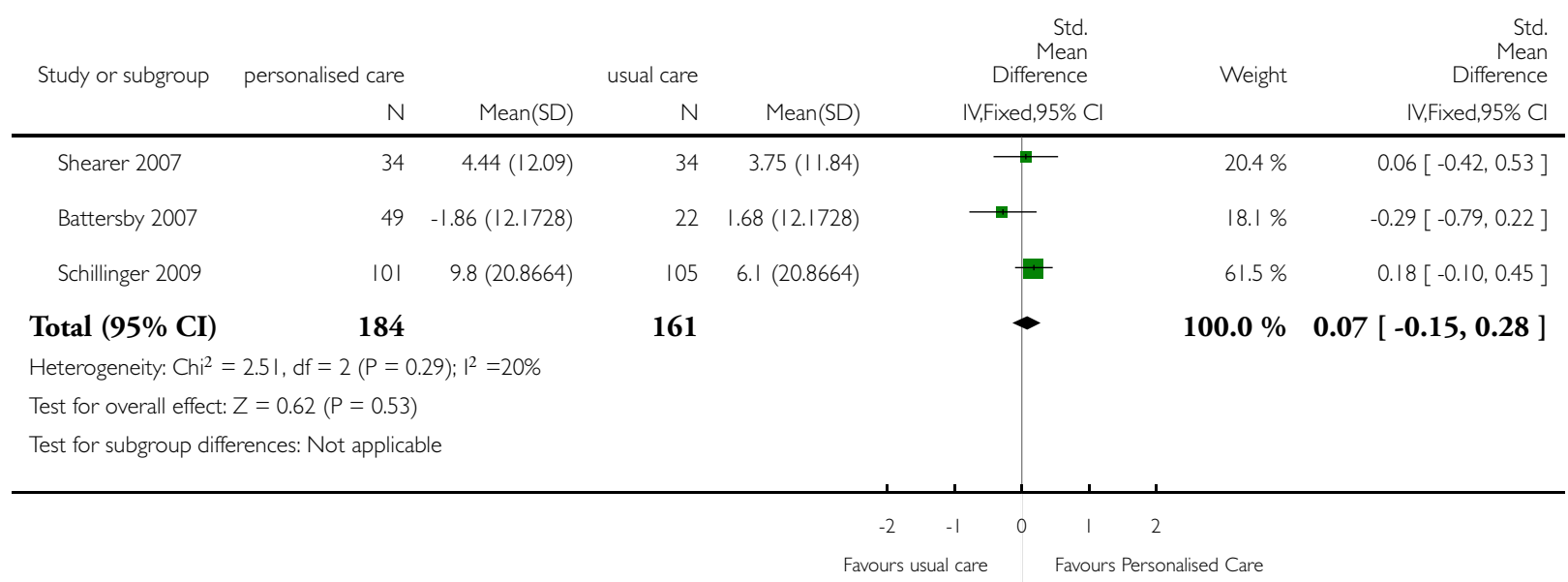


Analysis 3.3. Comparison 3 Subjective health status (personalised care planning vs usual care), Outcome 3 Condition-specific health status.

Review: Personalised care planning for adults with chronic or long-term health conditions

Comparison: 3 Subjective health status (personalised care planning vs usual care)

Outcome: 3 Condition-specific health status

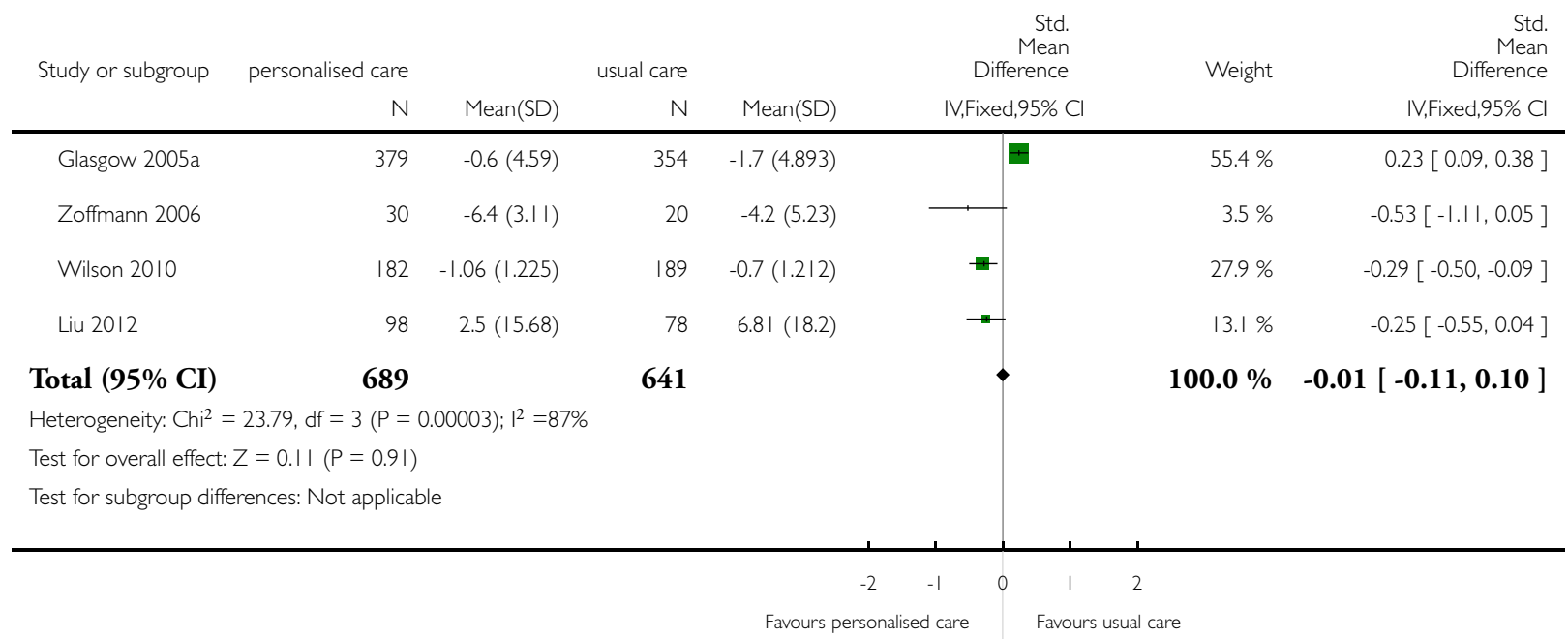


Analysis 4.I. Comparison 4 Self-management capabilities (personalised care planning vs usual care), Outcome I Self efficacy.

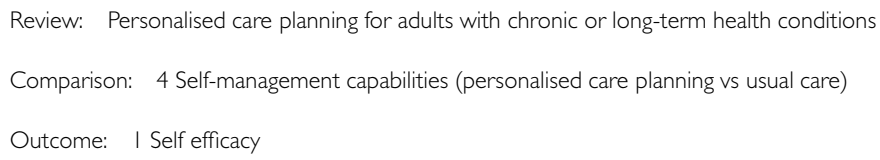

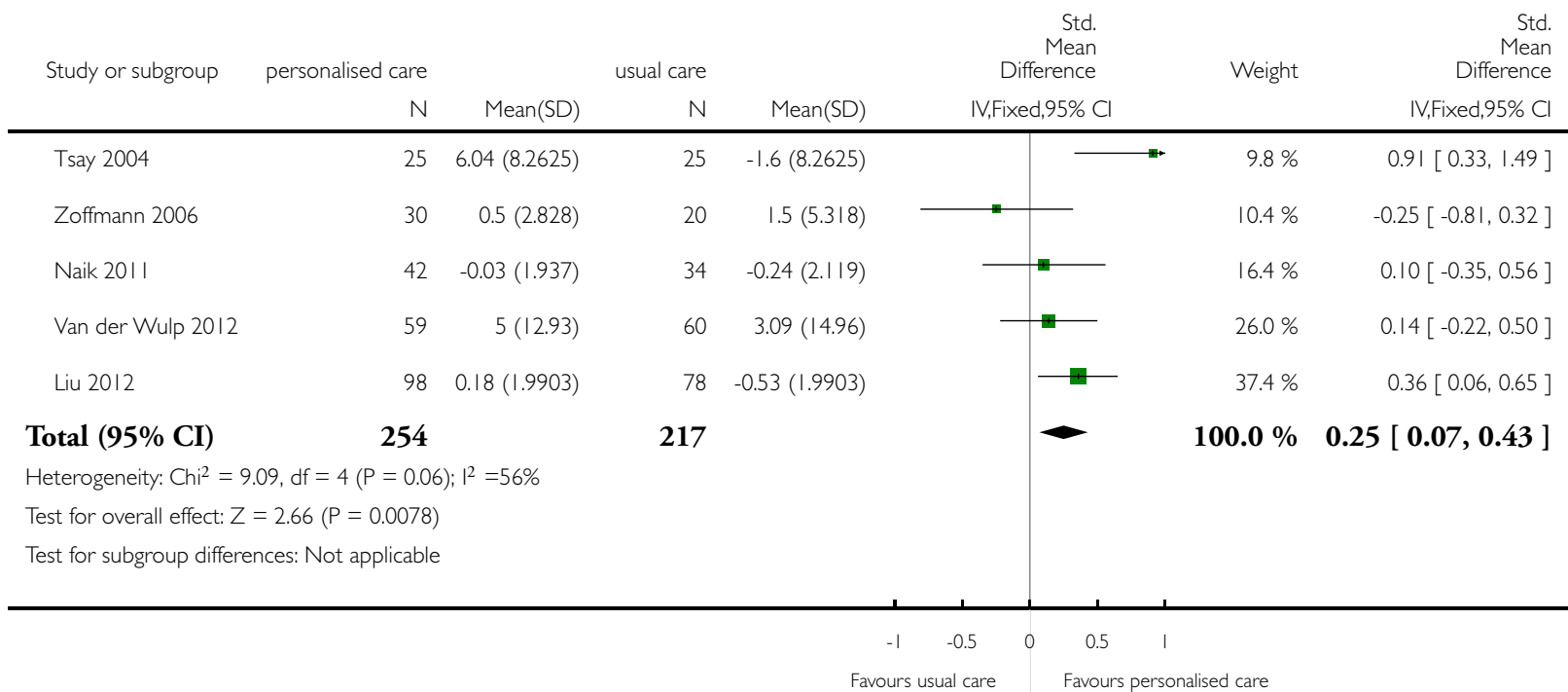




\section{Analysis 5.I. Comparison 5 Health-related behaviours (personalised care planning vs usual care), Outcome I Exercise.}

Review: Personalised care planning for adults with chronic or long-term health conditions

Comparison: 5 Health-related behaviours (personalised care planning vs usual care)

Outcome: I Exercise

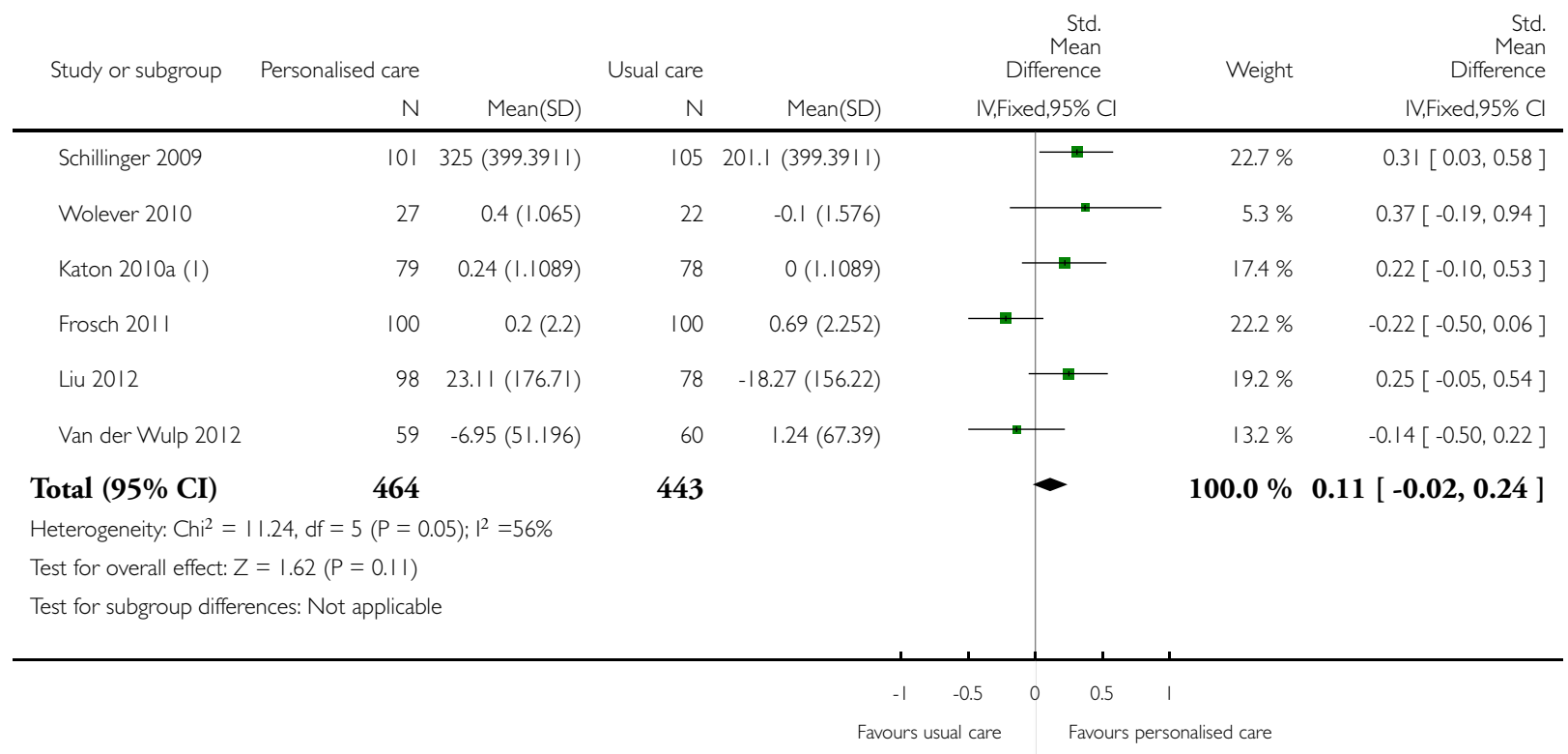

( I) Katon reported binary. Converted into SMD based on handbook transformation. 
Analysis 6.I. Comparison 6 Self-care activities (personalised care planning vs usual care), Outcome I Self care (days per week).

Review: Personalised care planning for adults with chronic or long-term health conditions

Comparison: 6 Self-care activities (personalised care planning vs usual care)

Outcome: I Self care (days per week)

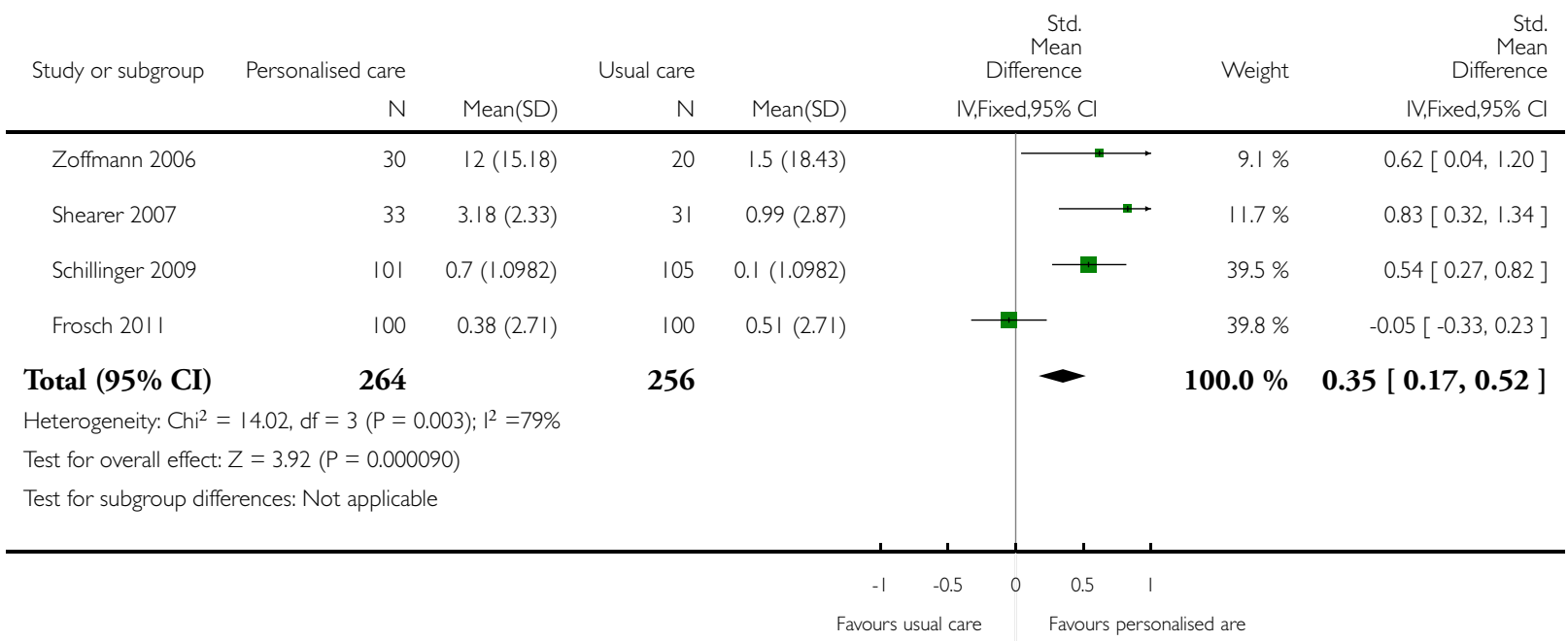




\section{Analysis 7.I. Comparison 7 Type of intervention (HbAlc) (extended vs limited), Outcome I HbAlc} (change).

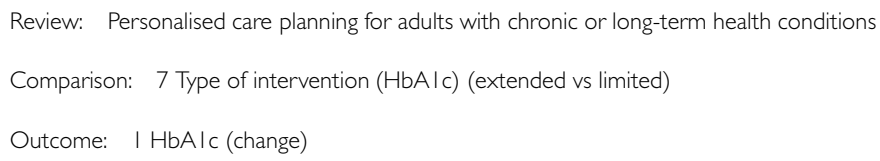

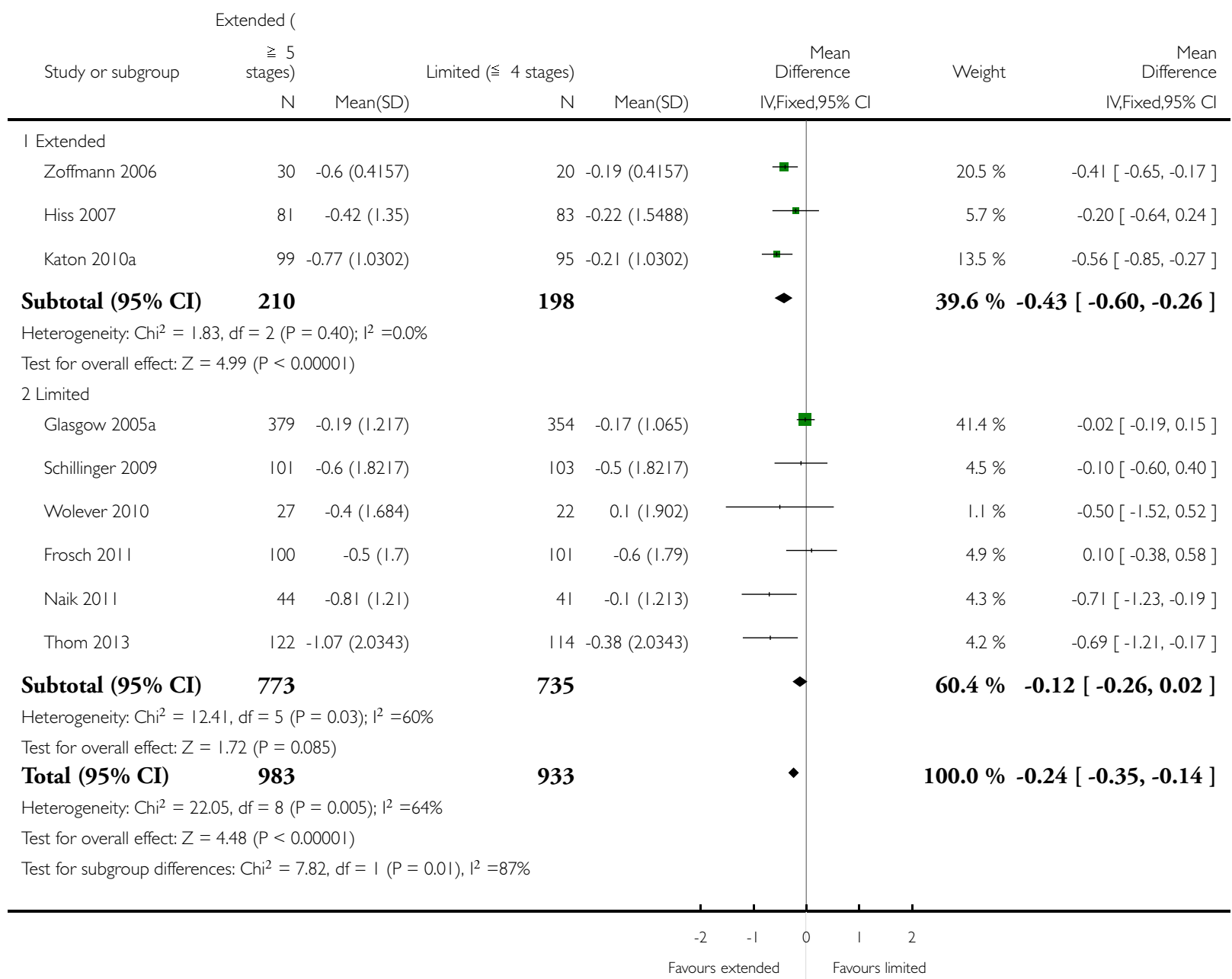




\section{Analysis 8.I. Comparison 8 Type of intervention (HbAIc) (high intensity vs low intensity), Outcome I}

HbAlc (change).

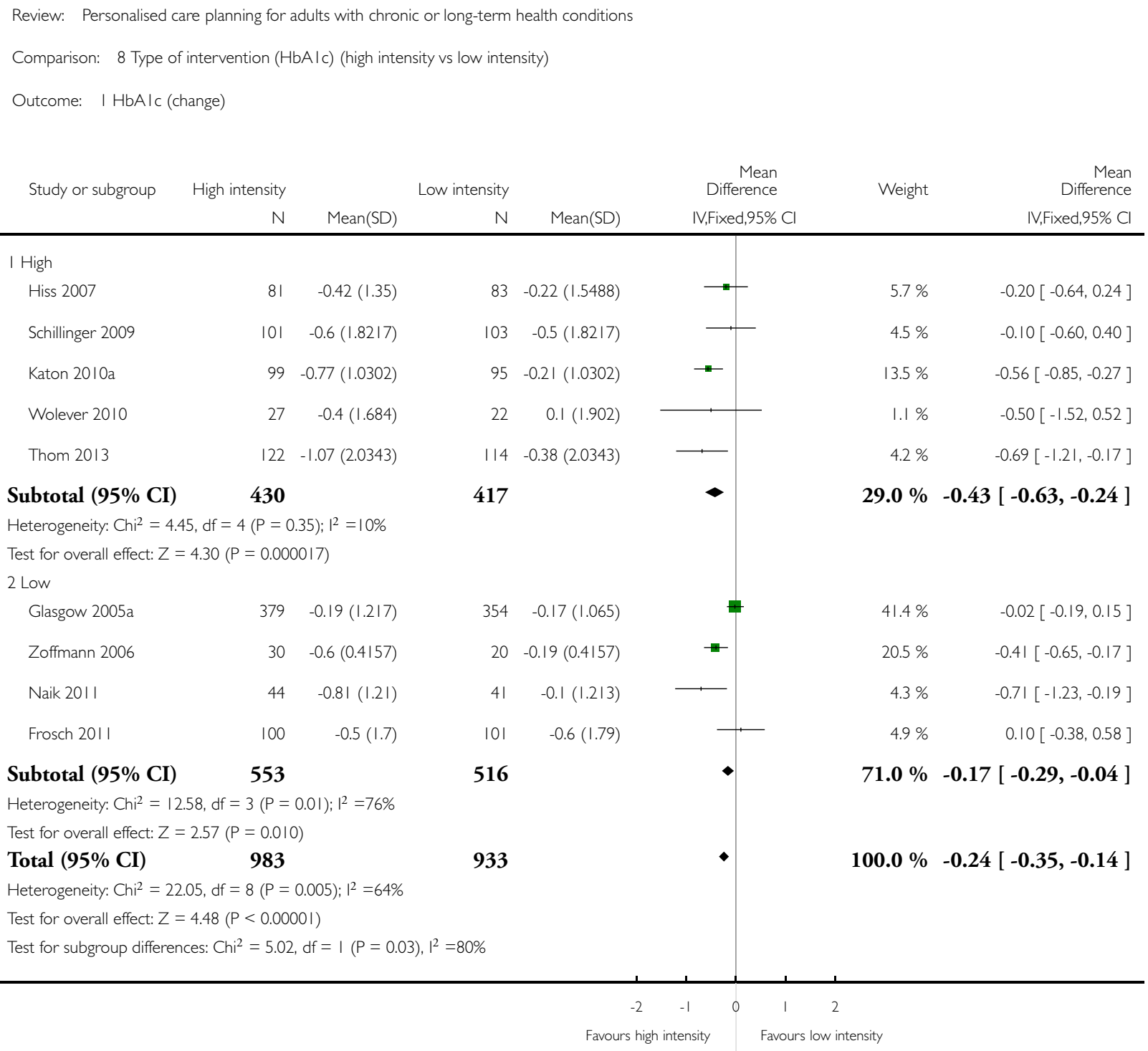




\section{Analysis 9.1. Comparison 9 Type of intervention (HbAlc) (integrated vs not integrated), Outcome I HbAlc (change).}

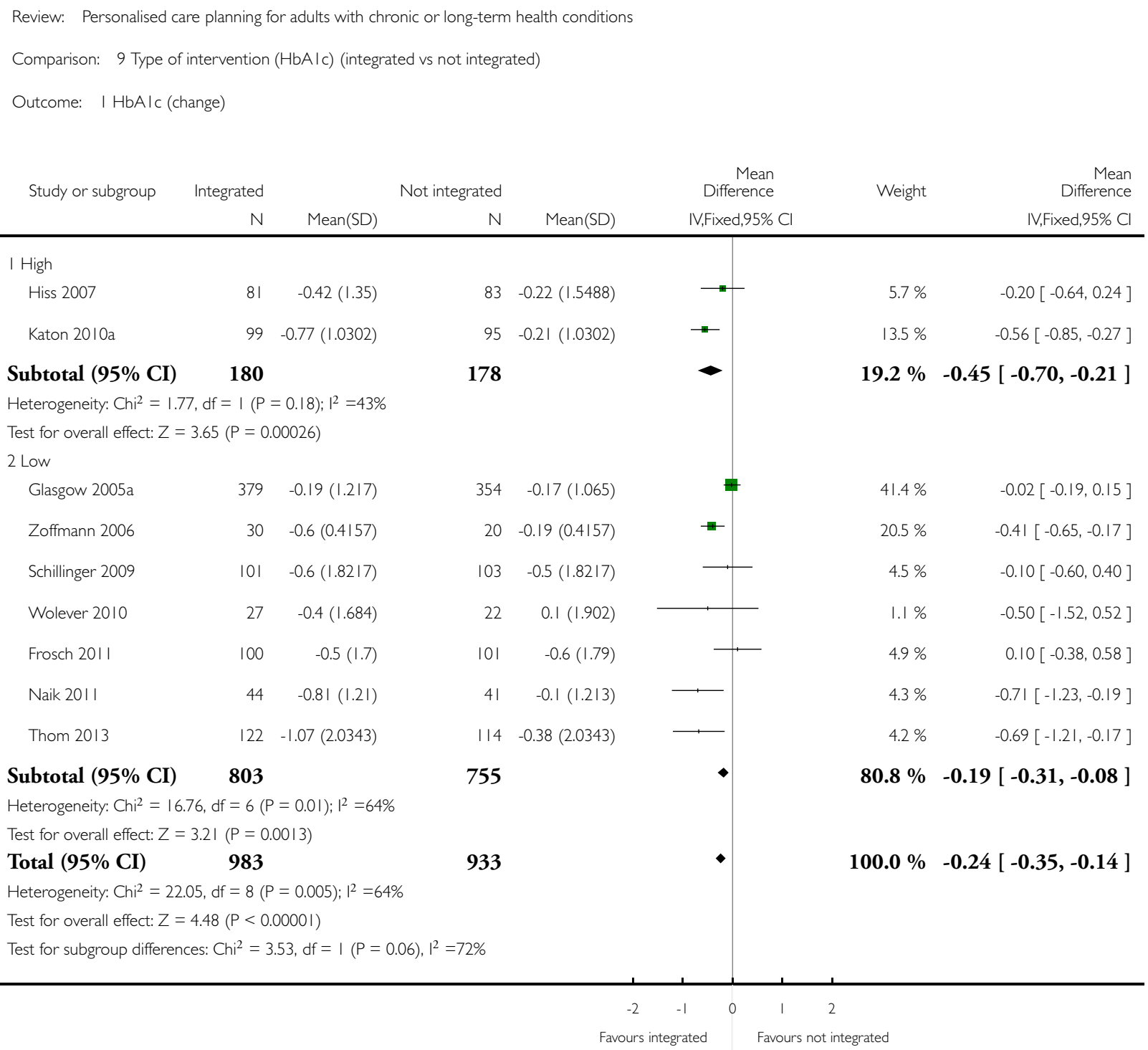


ADDITIONAL TABLES

Table 1. Included studies and interventions

\begin{tabular}{|c|c|c|c|c|c|c|c|c|}
\hline Study ID & $\begin{array}{l}\text { Type of } \\
\text { study }\end{array}$ & Country & Setting & $\begin{array}{l}\text { Condition } \\
\text { (s) }\end{array}$ & $\begin{array}{l}\text { No. of parti- } \\
\text { cipants }\end{array}$ & $\begin{array}{l}\text { Main focus } \\
\text { of interven- } \\
\text { tion }\end{array}$ & $\begin{array}{l}\text { Clinicians } \\
\text { involved } \\
\text { (usual/ } \\
\text { additional) }\end{array}$ & $\begin{array}{l}\text { Tools/ } \\
\text { techniques }\end{array}$ \\
\hline $\begin{array}{l}\text { Battersby } \\
2007\end{array}$ & RCT & Australia & Primary care & $\begin{array}{l}\text { Cardiac, res- } \\
\text { piratory, so- } \\
\text { matisation, } \\
\text { aged care }\end{array}$ & 1703 & $\begin{array}{l}\text { Both } \\
\text { patients and } \\
\text { clinicians }\end{array}$ & $\begin{array}{l}\text { Ser- } \\
\text { vice co-ordi- } \\
\text { nators (addi- } \\
\text { tional) and } \\
\text { GPs (usual) }\end{array}$ & $\begin{array}{l}\text { Problem \& } \\
\text { goals state- } \\
\text { ments, care } \\
\text { plan genera- } \\
\text { tor, face-to- } \\
\text { face contact }\end{array}$ \\
\hline $\begin{array}{l}\text { Frosch } \\
2011\end{array}$ & RCT & USA & Primary care & Diabetes & 201 & Patients & $\begin{array}{l}\text { Health } \\
\text { coach (addi- } \\
\text { tional) }\end{array}$ & $\begin{array}{l}\text { DVD + } \\
\text { booklet, } \\
\text { phone con- } \\
\text { tact }\end{array}$ \\
\hline $\begin{array}{l}\text { Glasgow } \\
2005 a\end{array}$ & $\begin{array}{l}\text { Cluster } \\
\text { RCT }\end{array}$ & USA & Primary care & Diabetes & 886 & Patients & $\begin{array}{l}\text { Care man- } \\
\text { agers (usual) }\end{array}$ & $\begin{array}{l}\text { CD-Rom } \\
\text { care en- } \\
\text { hancement } \\
\text { programme, } \\
\text { phone con- } \\
\text { tact }\end{array}$ \\
\hline Hart 1978 & RCT & USA & $\begin{array}{l}\text { Community } \\
\text { clinic }\end{array}$ & $\begin{array}{l}\text { Mental } \\
\text { health }\end{array}$ & 32 & Patients & $\begin{array}{l}\text { Clin- } \\
\text { ician 'scaler' } \\
\text { (addi- } \\
\text { tional) and } \\
\text { psychother- } \\
\text { apist (usual) }\end{array}$ & $\begin{array}{l}\text { Behavioural } \\
\text { monitor- } \\
\text { ing process } \\
\text { record, face- } \\
\text { to-face con- } \\
\text { tact }\end{array}$ \\
\hline Hiss 2007 & RCT & USA & Primary care & Diabetes & 197 & Patients & $\begin{array}{l}\text { Nurse care } \\
\text { manager } \\
\text { (additional) } \\
\text { and primary } \\
\text { care physi- } \\
\text { cian (usual) }\end{array}$ & $\begin{array}{l}\text { Structured } \\
\text { collabo- } \\
\text { ration, face- } \\
\text { to-face con- } \\
\text { tact }\end{array}$ \\
\hline $\begin{array}{l}\text { Katon } \\
2010 a\end{array}$ & RCT & USA & Primary care & $\begin{array}{l}\text { Depres- } \\
\text { sion + dia- } \\
\text { betes and/or } \\
\text { CHD }\end{array}$ & 214 & Patients & $\begin{array}{l}\text { Nurses (ad- } \\
\text { ditional), } \\
\text { primary care } \\
\text { physicians } \\
\text { (usual) }\end{array}$ & $\begin{array}{l}\text { DVD, } \\
\text { booklet, } \\
\text { self-moni- } \\
\text { toring de- } \\
\text { vices, face- } \\
\text { to-face con- } \\
\text { tact }\end{array}$ \\
\hline $\begin{array}{l}\text { Kennedy } \\
2013\end{array}$ & $\begin{array}{l}\text { Cluster } \\
\text { RCT }\end{array}$ & UK & Primary care & $\begin{array}{l}\text { Diabetes, } \\
\text { COPD, irri- }\end{array}$ & 5599 & $\begin{array}{l}\text { Both } \\
\text { patients and }\end{array}$ & $\begin{array}{l}\text { Nurses } \\
\text { (usual), GPs }\end{array}$ & $\begin{array}{l}\text { PRISMS } \\
\text { tool, book- }\end{array}$ \\
\hline
\end{tabular}

Personalised care planning for adults with chronic or long-term health conditions (Review) 
Table 1. Included studies and interventions (Continued)

\begin{tabular}{|c|c|c|c|c|c|c|c|c|}
\hline & & & & table bowel & & clinicians & (usual) & $\begin{array}{l}\text { lets, face-to- } \\
\text { face contact }\end{array}$ \\
\hline Liu 2012 & RCT & China & Primary care & Diabetes & 208 & Patients & $\begin{array}{l}\text { Nurse } \\
\text { (usual), GP } \\
\text { (usual), pre- } \\
\text { ventive doc- } \\
\text { tor (usual) }\end{array}$ & $\begin{array}{l}\text { Group edu- } \\
\text { ca- } \\
\text { tion + face- } \\
\text { to-face con- } \\
\text { tact }\end{array}$ \\
\hline $\begin{array}{l}\text { Ludman } \\
2007\end{array}$ & RCT & USA & Primary care & Depression & 52 & Patients & $\begin{array}{l}\text { Care } \\
\text { manager } \\
\text { (additional) }\end{array}$ & $\begin{array}{l}\text { Comput- } \\
\text { erised deci- } \\
\text { sion sup- } \\
\text { port, phone } \\
\text { contact }\end{array}$ \\
\hline Naik 2011 & RCT & USA & Primary care & Diabetes & 87 & Patients & $\begin{array}{l}\text { Physicians } \\
\text { (additional) }\end{array}$ & $\begin{array}{l}\text { Group edu- } \\
\text { ca- } \\
\text { tion + face- } \\
\text { to-face con- } \\
\text { tact }\end{array}$ \\
\hline $\begin{array}{l}\text { Schillinger } \\
2009\end{array}$ & RCT & USA & Primary care & Diabetes & 226 & Patients & $\begin{array}{l}\text { Care } \\
\text { manager } \\
\text { (additional) }\end{array}$ & $\begin{array}{l}\text { Automated } \\
\text { telephone } \\
+ \text { phone fol- } \\
\text { low-up }\end{array}$ \\
\hline $\begin{array}{l}\text { Shearer } \\
2007\end{array}$ & RCT & USA & $\begin{array}{l}\text { Hospital } \\
\text { clinic }\end{array}$ & Heart failure & 90 & Patients & $\begin{array}{l}\text { Nurses (ad- } \\
\text { ditional) }\end{array}$ & $\begin{array}{l}\text { Structured } \\
\text { collabo- } \\
\text { ration, face- } \\
\text { to-face con- } \\
\text { tact }\end{array}$ \\
\hline $\begin{array}{l}\text { Stanhope } \\
2013\end{array}$ & $\begin{array}{l}\text { Cluster } \\
\text { RCT }\end{array}$ & USA & $\begin{array}{l}\text { Community } \\
\text { clinic }\end{array}$ & $\begin{array}{l}\text { Mental } \\
\text { health }\end{array}$ & 367 & $\begin{array}{l}\text { Both } \\
\text { patients and } \\
\text { clinicians }\end{array}$ & $\begin{array}{l}\text { Mental } \\
\text { health } \\
\text { providers } \\
\text { (usual) }\end{array}$ & $\begin{array}{l}\text { Structured } \\
\text { collabo- } \\
\text { ration, face- } \\
\text { to-face con- } \\
\text { tact }\end{array}$ \\
\hline Thom 2013 & RCT & USA & Primary care & Diabetes & 299 & Patients & $\begin{array}{l}\text { Peer coaches } \\
\text { (additional) }\end{array}$ & $\begin{array}{l}\text { Structured } \\
\text { collabo- } \\
\text { ration, face- } \\
\text { to-face con- } \\
\text { tact }\end{array}$ \\
\hline Tsay 2004 & RCT & Taiwan & $\begin{array}{l}\text { Hospital } \\
\text { clinic }\end{array}$ & $\begin{array}{l}\text { End-stage } \\
\text { renal disease }\end{array}$ & 50 & Patients & $\begin{array}{l}\text { Nurse (addi- } \\
\text { tional) }\end{array}$ & $\begin{array}{l}\text { Informa- } \\
\text { tion + struc- } \\
\text { tured collab- } \\
\text { o- } \\
\text { ration, face- }\end{array}$ \\
\hline
\end{tabular}


Table 1. Included studies and interventions (Continued)

\begin{tabular}{|c|c|c|c|c|c|c|c|c|}
\hline & & & & & & & & $\begin{array}{l}\text { to-face con- } \\
\text { tact }\end{array}$ \\
\hline $\begin{array}{l}\text { Van der } \\
\text { Wulp } 2012\end{array}$ & RCT & Netherlands & Primary care & Diabetes & 119 & Patients & $\begin{array}{l}\text { Peer coaches } \\
\text { (additional) }\end{array}$ & $\begin{array}{l}\text { Structured } \\
\text { collabo- } \\
\text { ration, face- } \\
\text { to-face con- } \\
\text { tact }\end{array}$ \\
\hline $\begin{array}{l}\text { Wilson } \\
2010\end{array}$ & RCT & USA & Primary care & Asthma & 408 & $\begin{array}{l}\text { Both } \\
\text { patients and } \\
\text { clinicians }\end{array}$ & $\begin{array}{l}\text { Care man- } \\
\text { agers (usual) }\end{array}$ & $\begin{array}{l}\text { Informa- } \\
\text { tion + struc- } \\
\text { tured collab- } \\
\text { o- } \\
\text { ration, face- } \\
\text { to-face con- } \\
\text { tact }\end{array}$ \\
\hline $\begin{array}{l}\text { Wolever } \\
2010\end{array}$ & RCT & USA & $\begin{array}{l}\text { Community } \\
\text { clinic }\end{array}$ & Diabetes & 56 & Patients & $\begin{array}{l}\text { Health } \\
\text { coaches (ad- } \\
\text { ditional) }\end{array}$ & $\begin{array}{l}\text { Information } \\
+ \text { Wheel of } \\
\text { Health, } \\
\text { face-to-face } \\
\text { contact }\end{array}$ \\
\hline $\begin{array}{l}\text { Zoffmann } \\
2006\end{array}$ & RCT & Denmark & $\begin{array}{l}\text { Hospital } \\
\text { clinic }\end{array}$ & Diabetes & 61 & Patients & $\begin{array}{l}\text { Nurses (ad- } \\
\text { ditional) }\end{array}$ & $\begin{array}{l}\text { Group vis- } \\
\text { its + face-to- } \\
\text { face contact }\end{array}$ \\
\hline
\end{tabular}

CHD: coronary heart disease; COPD: chronic obstructive pulmonary disease

Table 2. Care planning cycle: stages completed

\begin{tabular}{|c|c|c|c|c|c|c|c|c|}
\hline Study ID & $\begin{array}{l}\text { Interven- } \\
\text { tion type }\end{array}$ & $\begin{array}{l}\text { A. Prepara- } \\
\text { tion }\end{array}$ & $\begin{array}{l}\text { B. goal set- } \\
\text { ting }\end{array}$ & $\begin{array}{l}\text { C. Action- } \\
\text { planning }\end{array}$ & $\begin{array}{l}\text { D. Docu- } \\
\text { menting }\end{array}$ & $\begin{array}{l}\text { E. Co-ordi- } \\
\text { nating }\end{array}$ & $\begin{array}{l}\text { F. Support- } \\
\text { ing }\end{array}$ & $\begin{array}{l}\text { G. Review- } \\
\text { ing }\end{array}$ \\
\hline $\begin{array}{l}\text { Battersby } \\
2007\end{array}$ & Extended & $\mathrm{X}$ & $\mathrm{X}$ & $\mathrm{X}$ & $\mathrm{X}$ & $\mathrm{X}$ & $\mathrm{X}$ & $\mathrm{X}$ \\
\hline $\begin{array}{l}\text { Frosch } \\
2011\end{array}$ & Limited & & $\mathrm{X}$ & $\mathrm{X}$ & & & $\mathrm{X}$ & \\
\hline $\begin{array}{l}\text { Glasgow } \\
2005 a\end{array}$ & Limited & & $\mathrm{X}$ & $\mathrm{X}$ & $\mathrm{X}$ & & $\mathrm{X}$ & \\
\hline Hart 1978 & Limited & & $\mathrm{X}$ & $\mathrm{X}$ & $\mathrm{X}$ & & $\mathrm{X}$ & \\
\hline Hiss 2007 & Extended & $X$ & $\mathrm{X}$ & $\mathrm{X}$ & & $X$ & $\mathrm{X}$ & \\
\hline $\begin{array}{l}\text { Katon } \\
2010 a\end{array}$ & Extended & $\mathrm{X}$ & $X$ & $\mathrm{X}$ & $\mathrm{X}$ & $\mathrm{X}$ & $\mathrm{X}$ & $\mathrm{X}$ \\
\hline
\end{tabular}

Personalised care planning for adults with chronic or long-term health conditions (Review)

Copyright $\odot 2015$ The Cochrane Collaboration. Published by John Wiley \& Sons, Ltd. 
Table 2. Care planning cycle: stages completed (Continued)

\begin{tabular}{|c|c|c|c|c|c|c|c|c|}
\hline $\begin{array}{l}\text { Kennedy } \\
2013\end{array}$ & Limited & & $\mathrm{X}$ & $\mathrm{X}$ & & $\mathrm{X}$ & $\mathrm{X}$ & \\
\hline Liu 2012 & Limited & & $\mathrm{X}$ & $\mathrm{X}$ & & & $\mathrm{X}$ & \\
\hline $\begin{array}{l}\text { Ludman } \\
2007\end{array}$ & Extended & & $\mathrm{X}$ & $\mathrm{X}$ & $\mathrm{X}$ & $\mathrm{X}$ & $\mathrm{X}$ & \\
\hline Naik 2011 & Limited & & $\mathrm{X}$ & $\mathrm{X}$ & & & $\mathrm{X}$ & $\mathrm{X}$ \\
\hline $\begin{array}{l}\text { Schillinger } \\
2009\end{array}$ & Limited & & $\mathrm{X}$ & $\mathrm{X}$ & & & $\mathrm{X}$ & \\
\hline $\begin{array}{l}\text { Shearer } \\
2007\end{array}$ & Limited & & $\mathrm{X}$ & $\mathrm{X}$ & & & $\mathrm{X}$ & \\
\hline $\begin{array}{l}\text { Stanhope } \\
2013\end{array}$ & Limited & & $\mathrm{X}$ & $\mathrm{X}$ & $\mathrm{X}$ & & $\mathrm{X}$ & \\
\hline Thom 2013 & Limited & & $\mathrm{X}$ & $\mathrm{X}$ & & & $\mathrm{X}$ & \\
\hline Tsay 2004 & Limited & & $\mathrm{X}$ & $\mathrm{X}$ & & & $\mathrm{X}$ & \\
\hline $\begin{array}{lr}\text { Van } & \text { der } \\
\text { Wulp } 2012\end{array}$ & Limited & & $\mathrm{X}$ & $\mathrm{X}$ & & & $\mathrm{X}$ & \\
\hline $\begin{array}{l}\text { Wilson } \\
2010\end{array}$ & Limited & & $\mathrm{X}$ & $\mathrm{X}$ & & & $\mathrm{X}$ & \\
\hline $\begin{array}{l}\text { Wolever } \\
2010\end{array}$ & Limited & $\mathrm{X}$ & $\mathrm{X}$ & $\mathrm{X}$ & & & $\mathrm{X}$ & \\
\hline $\begin{array}{l}\text { Zoffmann } \\
2006\end{array}$ & Extended & $\mathrm{X}$ & $\mathrm{X}$ & $\mathrm{X}$ & $\mathrm{X}$ & & $\mathrm{X}$ & \\
\hline
\end{tabular}

Table 3. Degree of intensity and integration of the care planning intervention

\begin{tabular}{|c|c|c|c|c|c|c|}
\hline Study ID & $\begin{array}{l}\text { Duration of in- } \\
\text { tervention }\end{array}$ & $\begin{array}{l}\text { Number of con- } \\
\text { tacts between } \\
\text { clinician (care } \\
\text { manager or } \\
\text { peer coach) and } \\
\text { participant }\end{array}$ & $\begin{array}{l}\text { Intensity } \\
\text { (1 or more con- } \\
\text { tacts per month } \\
\text { for more than } 3 \\
\text { months = high) }\end{array}$ & $\begin{array}{l}\text { Usual care clini- } \\
\text { cian involved in } \\
\text { care-planning } \\
\text { intervention }\end{array}$ & $\begin{array}{l}\text { Usual-care clin- } \\
\text { ician informed } \\
\text { about patient's } \\
\text { goals and plans }\end{array}$ & $\begin{array}{l}\text { In- } \\
\text { tegration (usual } \\
\text { clinician both } \\
\text { involved and in- } \\
\text { formed = high) }\end{array}$ \\
\hline Battersby 2007 & 12 months & 8 to 12 & High & Yes & Yes & Yes \\
\hline Frosch 2011 & 6 months & Up to 5 & Low & No & No & No \\
\hline
\end{tabular}


Table 3. Degree of intensity and integration of the care planning intervention (Continued)

\begin{tabular}{|c|c|c|c|c|c|c|}
\hline Glasgow 2005a & 6 months & 2 to 4 & Low & No & Yes & No \\
\hline Hart 1978 & 3 months & 3 & Low & Yes & Yes & Yes \\
\hline Hiss 2007 & 6 months & mean $=7$ & High & Yes & Yes & Yes \\
\hline Katon 2010a & 12 months & 16 to 24 & High & Yes & Yes & Yes \\
\hline Kennedy 2013 & 12 months & Not reported & Low & Yes & Yes & Yes \\
\hline Liu 2012 & 12 months & 12 & High & Yes & Yes & Yes \\
\hline Ludman 2007 & 12 months & 3 or more & Low & No & Yes & No \\
\hline Naik 2011 & 3 months & 4 & Low & No & Yes & No \\
\hline $\begin{array}{l}\text { Schillinger } \\
2009\end{array}$ & 9 months & 39 or more & High & No & Yes & No \\
\hline Shearer 2007 & 3 months & 6 & Low & No & No & No \\
\hline Stanhope 2013 & 11 months & Not reported & Low & Yes & Yes & Yes \\
\hline Thom 2013 & 6 months & $14+$ & High & No & No & No \\
\hline Tsay 2004 & 1 month & 12 & Low & No & No & No \\
\hline $\begin{array}{l}\text { Van der Wulp } \\
2012\end{array}$ & 3 months & $3+$ & High & No & No & No \\
\hline Wilson 2010 & 9 months & 4 & Low & Yes & Yes & Yes \\
\hline Wolever 2010 & 6 months & 14 & High & No & No & No \\
\hline Zoffmann 2006 & 12 months & $8+$ & Low & No & No & No \\
\hline
\end{tabular}

Table 4. Physical health

\begin{tabular}{|c|c|c|c|c|c|c|c|c|c|c|}
\hline Study & $\begin{array}{l}\text { Condi- } \\
\text { tion }\end{array}$ & $\begin{array}{l}\text { No. of } \\
\text { partici- } \\
\text { pants }\end{array}$ & $\begin{array}{l}\text { Timing } \\
\text { of } \\
\text { outcome } \\
\text { measure- } \\
\text { ment }\end{array}$ & HbAlc & SBP & DBP & LDL-C & BMI & Other & $\begin{array}{l}\text { Effects of interven- } \\
\text { tion } \\
\text { as reported }\end{array}$ \\
\hline $\begin{array}{l}\text { Frosch } \\
2011\end{array}$ & Diabetes & 201 & 6 months & $\mathrm{X}$ & $\mathrm{X}$ & $\mathrm{X}$ & $\mathrm{X}$ & $\mathrm{X}$ & & No significant effects \\
\hline
\end{tabular}


Table 4. Physical health (Continued)

\begin{tabular}{|c|c|c|c|c|c|c|c|c|c|c|}
\hline $\begin{array}{l}\text { Glasgow } \\
2005 a\end{array}$ & Diabetes & 886 & $\begin{array}{l}12 \\
\text { months }\end{array}$ & $\mathrm{X}$ & & & $\mathrm{X}$ & & & No significant effects \\
\hline $\begin{array}{l}\text { Hiss } \\
2007\end{array}$ & Diabetes & 197 & 6 months & $\mathrm{X}$ & $\mathrm{X}$ & $\mathrm{X}$ & $\mathrm{X}$ & & & $\begin{array}{l}\text { HbA1c improved } \\
\text { SBP improved }\end{array}$ \\
\hline $\begin{array}{l}\text { Katon } \\
2010 a\end{array}$ & $\begin{array}{l}\text { Depres- } \\
\text { sion }+ \\
\text { diabetes/ } \\
\text { CHD }\end{array}$ & 214 & $\begin{array}{l}12 \\
\text { months }\end{array}$ & $\mathrm{X}$ & $\mathrm{X}$ & & $\mathrm{X}$ & & & $\begin{array}{l}\text { HbA1c improved } \\
\text { SBP improved } \\
\text { LDL-C improved }\end{array}$ \\
\hline Liu 2012 & Diabetes & 208 & $\begin{array}{l}12 \\
\text { months }\end{array}$ & & $\mathrm{X}$ & $\mathrm{X}$ & & $\mathrm{X}$ & & SBP improved \\
\hline $\begin{array}{l}\text { Naik } \\
2011\end{array}$ & Diabetes & 87 & $\begin{array}{l}12 \\
\text { months }\end{array}$ & $\mathrm{X}$ & & & & & & HbA1c improved \\
\hline $\begin{array}{l}\text { Schillinger } \\
2009\end{array}$ & Diabetes & 226 & $\begin{array}{l}12 \\
\text { months }\end{array}$ & $\mathrm{X}$ & $\mathrm{X}$ & $\mathrm{X}$ & & $\mathrm{X}$ & & No significant effects \\
\hline $\begin{array}{l}\text { Thom } \\
2013\end{array}$ & Diabetes & 299 & 6 months & $\mathrm{X}$ & $\mathrm{X}$ & & $\mathrm{X}$ & $\mathrm{X}$ & & HbA1c improved \\
\hline $\begin{array}{l}\text { Wilson } \\
2010\end{array}$ & Asthma & 408 & $\begin{array}{l}12 \\
\text { months }\end{array}$ & & & & & & $\begin{array}{l}\text { FEV1, } \\
\text { ATAQ }\end{array}$ & $\begin{array}{l}\text { FEV1 improved } \\
\text { ATAQ improved }\end{array}$ \\
\hline $\begin{array}{l}\text { Wolever } \\
2010\end{array}$ & Diabetes & 56 & 6 months & $\mathrm{X}$ & & & & & & HbA1c improved \\
\hline $\begin{array}{l}\text { Zoff- } \\
\text { mann } \\
2006\end{array}$ & Diabetes & 61 & $\begin{array}{l}12 \\
\text { months }\end{array}$ & $X$ & & & & & & HbA1c improved \\
\hline
\end{tabular}

Table 5. Psychological health

\begin{tabular}{|c|c|c|c|c|c|c|}
\hline Study & Condition & $\begin{array}{l}\text { No. of partici- } \\
\text { pants }\end{array}$ & $\begin{array}{l}\text { Timing of out- } \\
\text { come } \\
\text { measurement }\end{array}$ & $\begin{array}{l}\text { Outcomes } \\
\text { included } \\
\text { in meta-analy- } \\
\text { sis }\end{array}$ & $\begin{array}{l}\text { Outcomes not } \\
\text { included } \\
\text { in meta-analy- } \\
\text { sis }\end{array}$ & $\begin{array}{l}\text { Results as re- } \\
\text { ported }\end{array}$ \\
\hline Glasgow 2005a & Diabetes & 886 & 12 months & PHQ-9 & & $\begin{array}{l}\text { No significant } \\
\text { effects }\end{array}$ \\
\hline
\end{tabular}


Table 5. Psychological health (Continued)

\begin{tabular}{|c|c|c|c|c|c|c|}
\hline Katon 2010a & $\begin{array}{l}\text { Depression + } \\
\text { diabetes/CHD }\end{array}$ & 214 & 12 months & SCL-20 & PGI & $\begin{array}{l}\text { Depression im- } \\
\text { proved }\end{array}$ \\
\hline Liu 2012 & Diabetes & 208 & 12 months & PHQ-9 & & $\begin{array}{l}\text { No significant } \\
\text { effects }\end{array}$ \\
\hline Ludman 2007 & Depression & 52 & 12 months & SCL-20 & PGI, SCID & $\begin{array}{l}\text { No significant } \\
\text { effects }\end{array}$ \\
\hline Tsay 2004 & $\begin{array}{l}\text { End-stage } \\
\text { renal disease }\end{array}$ & 50 & 6 weeks & $\begin{array}{l}\text { Beck Depression } \\
\text { Inventory }\end{array}$ & & $\begin{array}{l}\text { Depression im- } \\
\text { proved }\end{array}$ \\
\hline $\begin{array}{l}\text { Van der Wulp } \\
2012\end{array}$ & Diabetes & 119 & 6 months & CES-D & & $\begin{array}{l}\text { No significant } \\
\text { effects }\end{array}$ \\
\hline Wolever 2010 & Diabetes & 56 & 6 months & & $\begin{array}{l}\text { PSS-4, Benefit } \\
\text { Finding }\end{array}$ & $\begin{array}{l}\text { Stress improved } \\
\text { Benefit finding } \\
\text { improved }\end{array}$ \\
\hline
\end{tabular}

CES-D: Center for Epidemiologic Studies Depression scale; CHD: coronary heart disease; PGI: Patient Global rating for Improvement; PHQ-9: Patient Health Questionnaire; PSS-4: Perceived Stress Scale; SCID: Structured Clinical Interview for DSM-IV Depression; SCL-20: Symptom Checklist 20

Table 6. Subjective health status

\begin{tabular}{|c|c|c|c|c|c|c|c|}
\hline Study & Condition & $\begin{array}{l}\text { No. of partici- } \\
\text { pants }\end{array}$ & $\begin{array}{l}\text { Timing of } \\
\text { outcome } \\
\text { measurement }\end{array}$ & $\begin{array}{l}\text { Generic } \\
\text { health status }\end{array}$ & $\begin{array}{l}\text { Other generic } \\
\text { measures not } \\
\text { included in } \\
\text { meta-analysis }\end{array}$ & $\begin{array}{l}\text { Condition- } \\
\text { specific } \\
\text { health status }\end{array}$ & $\begin{array}{l}\text { Results as re- } \\
\text { ported }\end{array}$ \\
\hline $\begin{array}{l}\text { Battersby } \\
2007\end{array}$ & Various & $\begin{array}{l}1703 \text { (124 in- } \\
\text { cluded } \\
\text { in meta-analy- } \\
\text { sis) }\end{array}$ & 12 months & $\begin{array}{l}\text { SF-36 (PCS, } \\
\text { MCS) }\end{array}$ & WSAS & & $\begin{array}{l}\text { No significant } \\
\text { effects }\end{array}$ \\
\hline $\begin{array}{l}\text { Glasgow } \\
\text { 2005a }\end{array}$ & Diabetes & 886 & 12 months & & & PAID-2 & $\begin{array}{l}\text { No significant } \\
\text { effects }\end{array}$ \\
\hline Katon 2010a & $\begin{array}{l}\text { Depression + } \\
\text { diabetes/ } \\
\text { CHD }\end{array}$ & 214 & 12 months & & $\begin{array}{l}\text { WHODAS-2, } \\
\text { Sheehan } \\
\text { disability } \\
\text { scale, global } \\
\text { rating } \\
\text { score }\end{array}$ & & $\begin{array}{l}\text { Sheehan } \\
\text { improved } \\
\text { Global rating } \\
\text { improved }\end{array}$ \\
\hline $\begin{array}{l}\text { Kennedy } \\
2013\end{array}$ & $\begin{array}{l}\text { Diabetes, } \\
\text { COPD, IBS }\end{array}$ & 5599 & 12 months & & $\begin{array}{l}\text { Selected sub- } \\
\text { scales }\end{array}$ & & $\begin{array}{l}\text { No significant } \\
\text { effects }\end{array}$ \\
\hline
\end{tabular}

Personalised care planning for adults with chronic or long-term health conditions (Review) 
Table 6. Subjective health status (Continued)

\begin{tabular}{|c|c|c|c|c|c|c|c|}
\hline & & & & & $\begin{array}{l}\text { of SF-36, EQ- } \\
5 \mathrm{D}\end{array}$ & & \\
\hline Liu 2012 & Diabetes & 208 & 12 months & & & $\begin{array}{l}\text { Stanford } \\
\text { Illness } \\
\text { Intrusiveness }\end{array}$ & $\begin{array}{l}\text { Illness intru- } \\
\text { siveness } \\
\text { improved }\end{array}$ \\
\hline $\begin{array}{l}\text { Schillinger } \\
2009\end{array}$ & Diabetes & 226 & 12 months & $\begin{array}{l}\text { SF-12 (PCS, } \\
\text { MCS) }\end{array}$ & & & $\begin{array}{l}\text { No significant } \\
\text { effects }\end{array}$ \\
\hline Shearer 2007 & Heart failure & 90 & 3 months & $\begin{array}{l}\text { SF-36 (PCS, } \\
\text { MCS) }\end{array}$ & & & $\begin{array}{l}\text { No significant } \\
\text { effects }\end{array}$ \\
\hline Wilson 2010 & Asthma & 408 & 12 months & & & AQLQ & $\begin{array}{l}\text { AQLQ } \\
\text { improved }\end{array}$ \\
\hline Wolever 2010 & Diabetes & 56 & 6 months & & $\begin{array}{l}\text { SF-12 (single } \\
\text { score) }\end{array}$ & & $\begin{array}{l}\text { No significant } \\
\text { effects }\end{array}$ \\
\hline $\begin{array}{l}\text { Zoffmann } \\
2006\end{array}$ & Diabetes & 61 & 12 months & & & PAID-2 & $\begin{array}{l}\text { No significant } \\
\text { effects }\end{array}$ \\
\hline
\end{tabular}

Table 7. Self-management capabilities

\begin{tabular}{|c|c|c|c|c|c|c|}
\hline Study & Condition & $\begin{array}{l}\text { No. of partici- } \\
\text { pants }\end{array}$ & $\begin{array}{l}\text { Timing of out- } \\
\text { come } \\
\text { measurement }\end{array}$ & Self efficacy & $\begin{array}{l}\text { Other measures } \\
\text { not } \\
\text { included in } \\
\text { meta-analysis }\end{array}$ & $\begin{array}{l}\text { Results as re- } \\
\text { ported }\end{array}$ \\
\hline Frosch 2011 & Diabetes & 201 & 6 months & & $\begin{array}{l}\text { Michigan } \\
\text { Diabetes } \\
\text { Knowledge }\end{array}$ & $\begin{array}{l}\text { No significant } \\
\text { effects }\end{array}$ \\
\hline Katon 2010a & $\begin{array}{l}\text { Depression + } \\
\text { diabetes/CHD }\end{array}$ & 214 & 12 months & & $\begin{array}{l}4 \text { items from } \\
\text { PAM-13 }\end{array}$ & $\begin{array}{l}2 \text { PAM items } \\
\text { improved }\end{array}$ \\
\hline Kennedy 2013 & $\begin{array}{l}\text { Diabetes, } \\
\text { COPD, IBS }\end{array}$ & 5599 & 12 months & $\begin{array}{l}\text { Stanford } \\
\text { self efficacy }\end{array}$ & PEI & $\begin{array}{l}\text { No significant } \\
\text { effects }\end{array}$ \\
\hline Liu 2012 & Diabetes & 208 & 12 months & $\begin{array}{l}\text { Stanford } \\
\text { self efficacy }\end{array}$ & & $\begin{array}{l}\text { Self efficacy } \\
\text { improved }\end{array}$ \\
\hline
\end{tabular}

Personalised care planning for adults with chronic or long-term health conditions (Review) 
Table 7. Self-management capabilities (Continued)

\begin{tabular}{|c|c|c|c|c|c|c|}
\hline Naik 2011 & Diabetes & 87 & 12 months & $\begin{array}{l}\text { Stanford } \\
\text { self efficacy }\end{array}$ & & $\begin{array}{l}\text { Self efficacy } \\
\text { improved }\end{array}$ \\
\hline Shearer 2007 & Heart failure & 90 & 3 months & & РКРСТ & $\begin{array}{l}\text { No significant } \\
\text { effects }\end{array}$ \\
\hline Tsay 2004 & $\begin{array}{l}\text { End-stage } \\
\text { Renal Disease }\end{array}$ & 50 & 6 weeks & SUPPH & $\begin{array}{l}\text { Diabetes } \\
\text { Empowerment } \\
\text { Scale (DES) }\end{array}$ & $\begin{array}{l}\text { Self efficacy and } \\
\text { empowerment } \\
\text { improved }\end{array}$ \\
\hline $\begin{array}{l}\text { Van der Wulp } \\
2012\end{array}$ & Diabetes & 119 & 6 months & $\begin{array}{l}\text { Diabetes } \\
\text { self efficacy }\end{array}$ & & $\begin{array}{l}\text { No significant } \\
\text { effects }\end{array}$ \\
\hline Wolever 2010 & Diabetes & 56 & 6 months & & $\begin{array}{l}\text { ADS, ISEL-12, } \\
\text { PAM-13 }\end{array}$ & $\begin{array}{l}\text { ISEL-12 and } \\
\text { PAM-13 } \\
\text { improved }\end{array}$ \\
\hline Zoffmann 2006 & Diabetes & 61 & 12 months & PCDS & & PCDS improved \\
\hline
\end{tabular}

ADS: Appraisal of Diabetes Scale; CHD: coronary heart disease; CPOD: chronic obstructive pulmonary disease; IBS: irritable bowel syndrome; ISEL-12: Interpersonal Support Evaluation List; PAM-13: Patient Activation Measure; PCDS: Perceived Competence in Diabetes Scale; PEI: Patient Enablement Instrument; PKPCT: Power as Knowing Participation in Change Tool; SUPPH: Strategies Used by People to Promote Health

Table 8. Health behaviours

\begin{tabular}{|c|c|c|c|c|c|c|c|c|}
\hline Study & Condition & $\begin{array}{l}\text { No. of parti- } \\
\text { cipants }\end{array}$ & $\begin{array}{l}\text { Timing of } \\
\text { outcome } \\
\text { measure- } \\
\text { ment }\end{array}$ & Exercise & Diet & $\begin{array}{l}\text { Medication } \\
\text { adherence }\end{array}$ & $\begin{array}{l}\text { Self-care ac- } \\
\text { tivities }\end{array}$ & $\begin{array}{l}\text { Results as } \\
\text { reported }\end{array}$ \\
\hline $\begin{array}{l}\text { Frosch } \\
2011\end{array}$ & Diabetes & 201 & 6 months & $\begin{array}{l}\text { At least } 30 \\
\text { mins, } d / w k\end{array}$ & $\begin{array}{l}\text { General } \\
\text { diet, } \\
\text { d/wk }\end{array}$ & $\begin{array}{l}\text { Self report } \\
\text { (SDSCA) }\end{array}$ & $\begin{array}{l}\text { Blood glu- } \\
\text { cose testing, } \\
\text { foot care } \\
\text { (SDSCA } \\
\text { days/wk) }\end{array}$ & $\begin{array}{l}\text { Exercise im- } \\
\text { proved }\end{array}$ \\
\hline $\begin{array}{l}\text { Katon } \\
2010 a\end{array}$ & $\begin{array}{l}\text { Depression } \\
+ \\
\text { diabetes/ } \\
\text { CHD }\end{array}$ & 214 & 12 months & $\begin{array}{l}\text { At least } 30 \\
\text { mins, } \mathrm{d} / \mathrm{wk}\end{array}$ & $\begin{array}{l}\text { General } \\
\text { diet, } \\
\text { d/wk }\end{array}$ & $\begin{array}{l}\text { CMA phar- } \\
\text { macy refill }\end{array}$ & $\begin{array}{l}\text { Blood glu- } \\
\text { cose testing } \\
\text { and BP } \\
\text { monitoring } \\
\text { (days/wk)* }\end{array}$ & $\begin{array}{l}\text { Self care im- } \\
\text { proved }\end{array}$ \\
\hline Liu 2012 & Diabetes & 208 & 12 months & $\begin{array}{l}\text { Aerobic ex- } \\
\text { ercise } \\
\text { mins/wk }\end{array}$ & $\begin{array}{l}\text { Specific } \\
\text { questions, } \\
\text { no summary }\end{array}$ & & & $\begin{array}{l}\text { Exercise im- } \\
\text { proved }\end{array}$ \\
\hline
\end{tabular}

Personalised care planning for adults with chronic or long-term health conditions (Review) 
Table 8. Health behaviours (Continued)

\begin{tabular}{|c|c|c|c|c|c|c|c|c|}
\hline $\begin{array}{l}\text { Schillinger } \\
2009\end{array}$ & Diabetes & 226 & 12 months & $\begin{array}{l}\text { Moderate } \\
\text { activity, } \\
\text { mins/wk }\end{array}$ & & & $\begin{array}{l}\text { Self manage- } \\
\text { ment } \\
\text { days/wk }\end{array}$ & $\begin{array}{l}\text { Exercise im- } \\
\text { proved } \\
\text { Self care im- } \\
\text { proved }\end{array}$ \\
\hline $\begin{array}{l}\text { Shearer } \\
2007\end{array}$ & Heart failure & 90 & 3 months & & & & $\begin{array}{l}\text { Self manage- } \\
\text { ment } \\
\text { (SMHF - ex- } \\
\text { perience) }\end{array}$ & $\begin{array}{l}\text { Self care im- } \\
\text { proved }\end{array}$ \\
\hline $\begin{array}{l}\text { Stanhope } \\
2013\end{array}$ & $\begin{array}{l}\text { Mental } \\
\text { health }\end{array}$ & 367 & 11 months & & & $\begin{array}{l}\text { Clinician re- } \\
\text { port }\end{array}$ & & $\begin{array}{l}\text { Medication } \\
\text { adherence } \\
\text { improved }\end{array}$ \\
\hline $\begin{array}{lr}\text { Van der } \\
\text { Wulp } 2012\end{array}$ & Diabetes & 119 & 6 months & $\begin{array}{l}\text { Specific ac- } \\
\text { tivities, hrs/ } \\
\text { d (PASE) }\end{array}$ & $\begin{array}{l}\text { Saturated fat } \\
\text { intake } \\
\text { (Fatlist) }\end{array}$ & & & $\begin{array}{l}\text { No signifi- } \\
\text { cant effects }\end{array}$ \\
\hline $\begin{array}{l}\text { Wilson } \\
2010\end{array}$ & Asthma & 408 & 24 months & & & $\begin{array}{l}\text { CMA phar- } \\
\text { macy refill, } \\
\text { other adher- } \\
\text { ence } \\
\text { measures }\end{array}$ & & $\begin{array}{l}\text { Medication } \\
\text { adherence } \\
\text { improved }\end{array}$ \\
\hline $\begin{array}{l}\text { Wolever } \\
2010\end{array}$ & Diabetes & 56 & 6 months & $\begin{array}{l}\text { At least } 15 \\
\text { mins, } d / m t h\end{array}$ & & $\begin{array}{l}\text { Self report } \\
\text { (Morisky), } \\
\text { ASK-20 }\end{array}$ & & $\begin{array}{l}\text { Medica- } \\
\text { tion adher- } \\
\text { ence (ASK- } \\
20 \text { ) } \\
\text { improved }\end{array}$ \\
\hline $\begin{array}{l}\text { Zoffmann } \\
2006\end{array}$ & Diabetes & 61 & 12 months & & & & $\begin{array}{l}\text { Blood glu- } \\
\text { cose testing } \\
\text { (frequency) }\end{array}$ & $\begin{array}{l}\text { No signifi- } \\
\text { cant effects }\end{array}$ \\
\hline
\end{tabular}

* Excluded from meta-analysis because published data were incompletely reported

ASK-20: Adherence barrier questionnaire; CHD: coronary heart disease; CMA: continuous medication acquisition; PASE: Physical Activity Scale for the Elderly; SMHF: Self-Management of Heart Failure

Table 9. Resource use and costs

\begin{tabular}{|c|c|c|c|c|c|}
\hline Study & Condition & No. of participants & $\begin{array}{l}\text { Timing of outcome } \\
\text { measurement }\end{array}$ & Resource use & Results as reported \\
\hline Battersby 2007 & Various & $1703^{*}$ & 12 months & $\begin{array}{l}\text { Primary care, medi- } \\
\text { cations, } \\
\text { hospital admissions }\end{array}$ & $\begin{array}{l}\text { No reduction in ser- } \\
\text { vice use, no cost sav- } \\
\text { ings }\end{array}$ \\
\hline
\end{tabular}


Table 9. Resource use and costs (Continued)

\begin{tabular}{|c|c|c|c|c|c|}
\hline Katon 2010a & $\begin{array}{l}\text { Depression + } \\
\text { diabetes/CHD }\end{array}$ & 214 & 24 months & $\begin{array}{l}\text { Health plan } \\
\text { accounting records, } \\
\text { QALYs }\end{array}$ & $\begin{array}{l}0.335 \text { additional } \\
\text { QALYs for interven- } \\
\text { tion group and } \\
\text { lower outpatient } \\
\text { costs }\end{array}$ \\
\hline Wilson 2010 & Asthma & 408 & 12 months & Medications & $\begin{array}{l}\text { Significant changes } \\
\text { in medication use; no } \\
\text { assessment of cost ef- } \\
\text { fectiveness }\end{array}$ \\
\hline
\end{tabular}

* This figure includes 8 sub-regional studies, 4 of which used geographic controls and were not randomised.

CHD: coronary heart disease; QALY: quality-adjusted life year

\section{A P P E N D I C E S}

\section{Appendix I. Search strategy}

The cumulative search of electronic databases was as follows:

- Cochrane Central Register of Controlled Trials (CENTRAL, The Cochrane Library) (July 2013 Issue 7)

- Dissertations \& Theses (Proquest) (1743 - July 2013)

- Embase (Ovid) (1974 to July 2013)

- Medline \& Medline In-process (Ovid) (1946 to July 2013)

- PsycINFO (Ovid) (1967 to July 2013)

- Clinicaltrials.gov (21st June 2013)

\section{Search results}

\begin{tabular}{|c|c|c|c|c|}
\hline Database: & Interface: & Coverage: & Dates: & Hits: \\
\hline $\begin{array}{l}\text { Cochrane Central Regis- } \\
\text { ter of Controlled Trials }\end{array}$ & Cochrane Library, Wiley & & $31 / 07 / 2013$ & 2351 \\
\hline Dissertations \& Theses & Proquest & 1743 - present & $31 / 07 / 2013$ & 657 \\
\hline Embase & OvidSP & 1974 - present & $31 / 07 / 2013$ & 6811 \\
\hline $\begin{array}{l}\text { Medline \& Medline In- } \\
\text { process }\end{array}$ & OvidSP & 1946 - present & $31 / 07 / 2013$ & 4806 \\
\hline
\end{tabular}


(Continued)

\begin{tabular}{|c|c|c|c|c|}
\hline PsycINFO & OvidSP & 1967 - present & $31 / 07 / 2013$ & 1526 \\
\hline Final: & & & & 16151 \\
\hline Duplicates: & & & & 6273 \\
\hline Final total: & & & & 9878 \\
\hline
\end{tabular}

\section{CENTRAL:}

\#1 (chronic*):ti,ab,kw (Word variations have been searched)

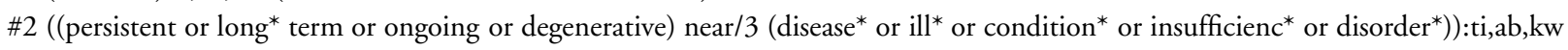
(Word variations have been searched)

\#3 ((longterm or long-term or "long term") next care):ti,ab,kw (Word variations have been searched)

\#4 MeSH descriptor: [Cardiovascular Diseases] explode all trees

\#5 "heart disease*" or "heart failure" or "myocardial ischemia" or "coronary disease" * or "coronary artery disease*" or "myocardial infarct"” or hypertension or "high blood pressure":ti,ab,kw (Word variations have been searched)

\#6 (“cardiovascular disease"” or cvd):ti,ab,kw (Word variations have been searched)

\#7 “sickle cell”:ti,ab,kw (Word variations have been searched)

\#8 MeSH descriptor: [Lung Diseases, Obstructive] explode all trees

\#9 “obstructive lung disease*” or "obstructive pulmonary disease*” or copd or asthma or bronchitis:ti,ab,kw (Word variations have been searched)

\#10 emphysema:ti,ab,kw (Word variations have been searched)

\#11 "cystic fibrosis" or "respiratory distress":ti,ab,kw (Word variations have been searched)

\#12 MeSH descriptor: [Nervous System Diseases] explode all trees

\#13 (brain next (disease* or damage* or injur*)):ti,ab,kw (Word variations have been searched)

\#14 cerebrovascular or "brain isch?emia" or "cerebral infarc*" or "carotid artery disease*” or stroke or epilep* or seizure*:ti,ab,kw (Word variations have been searched)

\#15 neurodegenerative or Huntington* or Parkinson* or "amyotrophic lateral sclerosis" or "multiple sclerosis" or "motor neuron disease":ti,ab,kw (Word variations have been searched)

\#16 paralys* or quadriplegi* or tetraplegi* or paraplegi* or "locked-in syndrome":ti,ab,kw (Word variations have been searched)

\#17 ((communication or learning or consciousness or perceptual or speech or voice or vision or hearing or psychomotor) next disorder*): ti,ab,kw (Word variations have been searched)

\#18 "hearing loss" or "hearing aid*” or deaf* or blind* or stutter*:ti,ab,kw (Word variations have been searched)

\#19 "down* syndrome" or "cerebral palsy":ti,ab,kw (Word variations have been searched)

\#20 MeSH descriptor: [Gastrointestinal Diseases] explode all trees

\#21 gastroenter* or intestinal or bowel or colonic:ti,ab,kw (Word variations have been searched)

\#22 ((renal or kidney) next (failure* or insufficienc*)):ti,ab,kw (Word variations have been searched)

\#23 (diabetes or diabetic*):ti,ab,kw (Word variations have been searched)

\#24 MeSH descriptor: [Nutrition Disorders] explode all trees

\#25 underweight or malnutrition or malnourished or overweight or obes*:ti,ab,kw (Word variations have been searched)

\#26 arthritis or osteoarthritis or rheumati* or fibromyalgia:ti,ab,kw (Word variations have been searched)

\#27 ((back or neck) adj pain):ti,ab,kw (Word variations have been searched)

\#28 MeSH descriptor: [Thyroid Diseases] explode all trees

\#29 thyroid:ti,ab,kw (Word variations have been searched)

\#30 MeSH descriptor: [Hypersensitivity] explode all trees

\#31 allerg* $^{*}$ or hypersensitivit* or tierg* or intolerance or anaphyla*:ti,ab,kw (Word variations have been searched)

\#32 MeSH descriptor: [Neoplasms] explode all trees

\#33 cancer* or oncolog* or neoplasm* or carcinom* or tumo? ${ }^{*}$ or malignan* or leuk?emia:ti,ab,kw (Word variations have been searched) \#34 MeSH descriptor: [HIV Infections] explode all trees

Personalised care planning for adults with chronic or long-term health conditions (Review) 
\#35 ("hiv infect*” or "hiv disease*”):ti,ab,kw (Word variations have been searched)

\#36 MeSH descriptor: [Mental Disorders] explode all trees

\#37 MeSH descriptor: [Behavioral Symptoms] explode all trees

\#38 ((mental* or psychiatr* or psychological* or behavio*) next (ill* or disorder* or disease* or distress* or disab* ${ }^{*}$ or problem* or health* or patient* or treatment)):ti,ab,kw (Word variations have been searched)

\#39 (psychosis or psychoses or psychotic* or paranoi* or schizo* or neurosis or neuroses or neurotic* or delusion* or depression or depressive or bipolar or mania or manic or obsessi* or compulsi* or panic or phobic or phobia or anorexia or bulimia or neurastheni* or dissociative or autis* or Asperger* or Tourette or dyslex* or affective or borderline or narcissis* or suicid* or self injur* or self harm or adhd):ti,ab,kw (Word variations have been searched)

\#40 ((personality or mood or dysthymic or cognit* or anxiety or stress or eating or adjustment or reactive or somatoform or conversion or behavior or perception or psycho* or "impulse control" or development* or "attention deficit" or hyperactivity or conduct or "motor skills" or movement or tic or "substance related") next disorder*):ti,ab,kw (Word variations have been searched)

\#41 (((substance or drug or alcohol) next abuse) or "substance use" or "illegal drug use" or addict* or alcoholism or (problem* near/1 drinking)):ti,ab,kw (Word variations have been searched)

$\# 42 \# 1$ or $\# 2$ or $\# 3$ or $\# 4$ or $\# 5$ or \#6 or \#7 or \#8 or \#9 or \#10 or \#11 or \#12 or \#13 or \#14 or \#15 or \#16 or \#17 or \#18 or \#19 or \# 20 or \#21 or \#22 or \#23 or \#24 or \#25 or \#26 or \#27 or \#28 or \#29 or \#30 or \#31 or \#32 or \#33 or \#34 or \#35 or \#36 or \#37 or \#38 or \#39 or \#40 or \#41

\#43 MeSH descriptor: [Patient Care Planning] this term only

\#44 ((care or action or treatment) next plan*):ti,ab,kw (Word variations have been searched)

\#45 MeSH descriptor: [Decision Making] this term only

\#46 MeSH descriptor: [Choice Behavior] this term only

\#47 (patient* near/7 (decision* or choice*)):ti,ab,kw (Word variations have been searched)

\#48 (patient* near/3 (preference* or priorit* or value*)):ti,ab,kw (Word variations have been searched)

\#49 MeSH descriptor: [Patient Preference] explode all trees

\#50 (treatment next (option* or choice*)):ti,ab,kw (Word variations have been searched)

\#51 MeSH descriptor: [Goals] explode all trees

\#52 (goal* adj2 (set* or plan*)):ti,ab,kw (Word variations have been searched)

\#53 MeSH descriptor: [Patient-Centered Care] explode all trees

\#54 (patient next (cent ${ }^{*}$ red or focus*ed or oriented)):ti,ab,kw (Word variations have been searched)

\#55 MeSH descriptor: [Individualized Medicine] explode all trees

\#56 (individualise? or individualize? or individualising or individualizing or personalise? or personalize? or personalising or personalizing or tailor or tailored or tailoring):ti,ab,kw (Word variations have been searched)

$\# 57 \# 43$ or \#44 or \#45 or \#46 or \#47 or \#48 or \#49 or \#50 or \#51 or \#52 or \#53 or \#54 or \#55 or \#56

\#58 (patient* near/3 (participat* or involv*)):ti,ab,kw (Word variations have been searched)

\#59 (negotiat* or agreement or concordan* or cooperat* ${ }^{*}$ or co-operat* or collaborat* or partnership):ti,ab,kw (Word variations have been searched)

\#60 \#58 or \#59

\#61 \#57 and \#60

\#62 MeSH descriptor: [Patient Participation] explode all trees

\#63 (patient* near/2 (empower* or activat*)):ti,ab,kw (Word variations have been searched)

\#64 ((shared or joint or informed or collaborative) near/2 decision making):ti,ab,kw (Word variations have been searched)

\#65 ((involv* or participat*) near/3 (choice* or decision*)):ti,ab,kw (Word variations have been searched)

\#66 (decision next (aid* or support or tool*)):ti,ab,kw (Word variations have been searched)

\#67 ("patient provider agreement*” or "decisional self efficacy" or "personal budget*” or "direct payment*” or "record access" or "patient held record*”):ti,ab,kw (Word variations have been searched)

\#68 ((“self management” or "self care”) near/2 support*):ti,ab,kw (Word variations have been searched)

$\# 69 \# 61$ or \#62 or \#63 or \#64 or \#65 or \#66 or \#67 or \#68

\#70 \#42 and \#69

Dissertations \& Theses:

Personalised care planning for adults with chronic or long-term health conditions (Review) 
(((all((chronic NEAR/3 (disease* OR ill* OR condition* OR insufficienc* OR disorder*))) OR all((longterm NEAR/3 (disease* OR ill* OR condition* OR insufficienc* OR disorder*))) OR all((long-term NEAR/3 (disease* OR ill* OR condition* OR insufficienc* OR disorder*))) OR all((persistent NEAR/3 (disease* OR ill* OR condition* OR insufficienc* OR disorder*))) OR all((ongoing NEAR/3 (disease* OR ill* OR condition* OR insufficienc* OR disorder*))) OR all((chronic NEAR/3 (disease* OR ill* OR condition* OR insufficienc* OR disorder*))) ) OR All(cardiovascular disease* or heart disease* or heart failure or myocardial ischemia or coronary disease* or coronary artery disease* or myocardial infarction or hypertension or high blood pressure) OR All(obstructive lung disease* or obstructive pulmonary disease* or copd or asthma or bronchitis or emphysema or "cystic fibrosis" or respiratory distress) OR All(brain disease* $^{*}$ or brain damage* or brain injur ${ }^{*}$ ) OR All(cerebrovascular or brain ischemia or cerebral infarction or carotid artery disease* or stroke or epilep* or seizure or Huntington* or Parkinson* or amyotrophic lateral sclerosis or multiple sclerosis or motor neuron disease or paralys* or quadriplegi* or tetraplegi* or paraplegi* or locked-in syndrome) OR All((disorder NEAR1 (communication or learning or consciousness or perceptual or speech or voice or vision or hearing or psychomotor))) OR All(hearing loss or hearing aid* or deaf* or blind* or stutter*) OR All(down* syndrome or cerebral palsy) OR All(gastroenter* or intestinal or bowel or colonic) OR All(gastroenter* or intestinal or bowel or colonic) OR All(diabetes or diabetic*) OR All(underweight or malnutrition or malnourished or overweight or obes*) OR All(arthritis or osteoarthritis or rheumati* or fibromyalgia or back pain or neck pain) OR All(thyroid*) OR All(hypersensitivit* or allerg* or intolerance or anaphyla*) OR All(cancer* or oncolog* or neoplasm* or carcinom* or tumo? ${ }^{*}{ }^{*}$ or malignan* or leuk?emia) OR All(hiv infect* or hiv disease*) OR All("mental illness" OR "mentally ill” OR "mental disorder*” OR "mental disease* OR "mental distress" OR "mental disab*” OR "mentally disabled” OR "mental problem*” OR " $m e n t a l$ health" OR "mental patient*” OR "mental treatment”) OR All(“psychiatric illness” OR "psychiatrically ill” OR "psychiatric disorder*” OR "psychiatric disease* OR "psychiatric distress" OR "psychiatric disab*” OR "psychiatrically disabled” OR "psychiatric problem*” OR "psychiatric health” OR "psychiatric patient”" OR "psychiatric treatment”) OR All(personality disorder* or mood disorder* or dysthymic disorder* or cognit* disorder* or anxiety disorder* or stress disorder* or eating disorder* or adjustment disorder* or reactive disorder* or somatoform disorder* conversion disorder* or behaviour* disorder* or perception disorder* or psycho* disorder* or impulse control disorder* or development* disorder* or attention deficit disorder* or hyperactivity disorder* or conduct disorder* or motor skills or movement disorder* or tic disorder* or substance related) OR All(psychosis or psychotic* or paranoi* or schizo* $^{*}$ or neuros\#s or neurotic* or delusion* or depression or depressive or bipolar or mania or manic or obsessi* or compulsi* or panic or phobic or phobia or anorexia or bulimia or neurastheni* or dissociative or autis* or Asperger* or Tourette or dyslex* or affective or borderline or narcissis* or suicid* or self injur* or self harm or adhd or psychoses) OR All(substance abuse or drug abuse or alcohol abuse or "substance use" or "illegal drug use" or addict* or alcoholism or problem drinking)) AND (((All((plan* NEAR/1 (care or action or treatment))) OR All((patient* NEAR/7 (decision* or choice*))) OR All((patient* NEAR/3 (preference* or priorit* or value*) )) OR All((treatment NEAR/1 (option* or choice*))) OR All((goal* NEAR/2 (set* or plan*))) OR All((patient NEAR/1 (cent*red or focus*ed or oriented))) OR All(individuali\#e? or individuali\#ing or personali\#e? or personali\#ing or tailor or tailored or tailoring) ) AND (All((patient* NEAR/3 (participat* or involv*))) OR All(negotiat* or agreement or concordan* or cooperat* or co-operat* or collaborat* or partnership))) OR All((patient* NEAR/2 (empower* or activat*))) OR All((decision making NEAR/2 (shared or joint or informed or collaborative)) ) OR (All((involv* NEAR/3 (choice* or decision*))) or All((participat* NEAR/3 (choice* or decision*) )) OR All(decision aid* or decision support or decision tool*) OR All(patient provider agreement* or decisional self efficacy or personal budget* or direct payment* or record access or patient held record*) OR All((support AND (self management or self care)) )) AND (ti((random* OR placebo* OR double blind*)) OR ab((random* OR placebo* OR double blind*)))

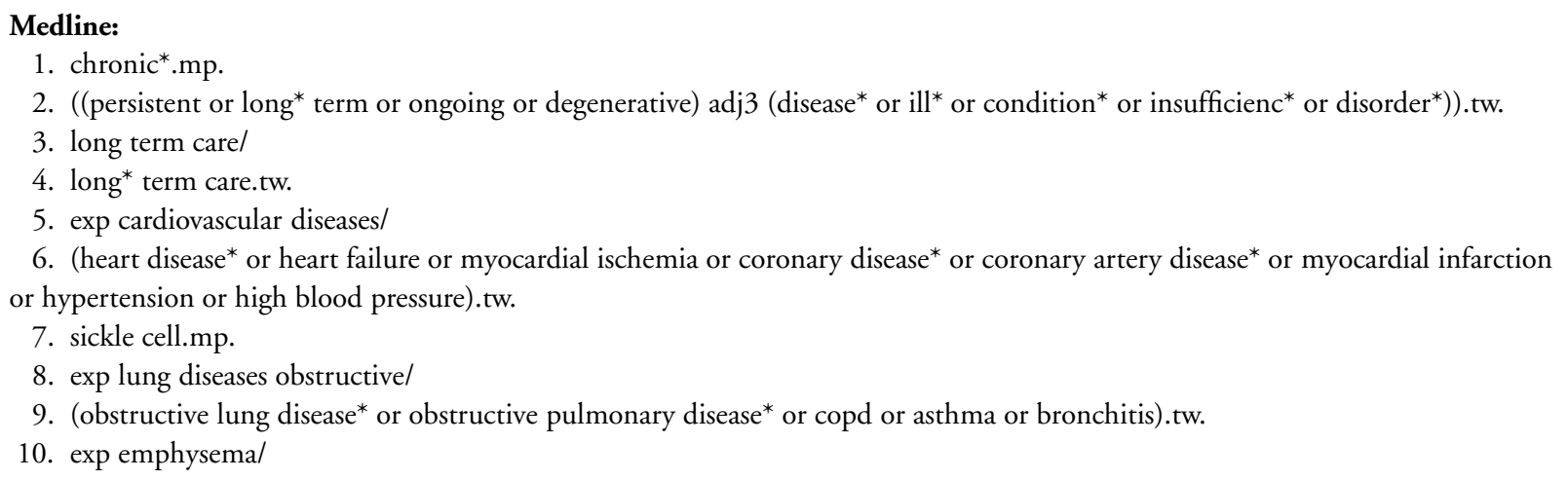

Personalised care planning for adults with chronic or long-term health conditions (Review)

Copyright () 2015 The Cochrane Collaboration. Published by John Wiley \& Sons, Ltd. 
11. exp pulmonary emphysema/

12. emphysema.tw.

13. (cystic fibrosis or respiratory distress), mp.

14. exp nervous system diseases/

15. (brain adj (disease* or damage* or injur*)).tw.

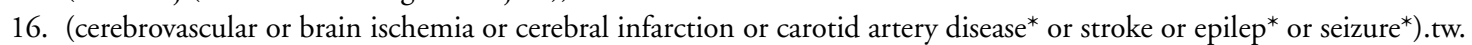

17. (neurodegenerative or Huntingdon* or Parkinson* or amyotrophic lateral sclerosis or multiple sclerosis or motor neuron disease).tw.

18. (paralys* or quadriplegi* or tetraplegi* or paraplegi* or locked-in syndrome).tw.

19. ((communication or learning or consciousness or perpetual or speech or voice or vision or hearing or psychomotor) adj disorder*).tw.

20. (hearing loss or hearing aid* or deaf* or blind* or stutter*).tw.

21. down* syndrome.tw.

22. cerebral palsy.tw.

23. exp gastrointestinal diseases/

24. (gatroenter* or intestinal or bowel or colonic).tw.

25. renal insufficiency/

26. ((renal or kidney) adj (failure* or insufficienc $\left.{ }^{*}\right)$ ).tw.

27. diabetes mellitus/

28. (diabetes or diabetic*).tw.

29. exp nutrition disorders/

30. (underweight or malnutrition or malnourished or overweight or obes*).tw.

31. exp arthritis/

32. exp rheumatic diseases/

33. (arthritis or osteoarthritis or rheumati* or fibromyalgia).tw.

34. ((back or neck) adj pain).tw.

35. exp thyroid diseases/

36. thyroid.tw.

37. exp hypersensitivity/

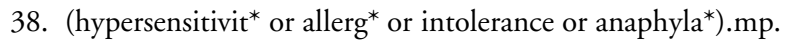

39. exp neoplasms/

40. (cancer* or oncolog* or neoplasm* or carcinom* or tumo? ${ }^{*}$ or malignan* or leuk?emia).tw.

41. exp hiv infections/

42. (hiv infect* or hiv disease*).tw.

43. exp mental disorders/

44. exp behavioral symptoms/

45. ((mental* or psychiatr* or psychological*) adj (ill* or disorder* or disease* or distress* ${ }^{*}$ or disab* or problem* or health* or patient* or treatment)).tw.

46. ((personality or mood or dysthymic or cognit* or anxiety or stress or eating or adjustment or reactive or somatoform or conversion or behavior or perception or psycho* or impulse control or development* or attention deficit or hyperactivity or conduct or motor skills or movement or tic or substance related) adj disorder*).tw.

47. (psychos\#s or psychotic* or paranoi* or schizo* or neuros\#s or neurotic* or delusion* or depression or depressive or bipolar or mania or manic or obsessi* or compulsi* or panic or phobic or phobia or anorexia or bulimia or neurastheni* or dissociative or autis* or Asperger* or Tourette or dyslex* or affective or borderline or narcissis* or suicid* or self injur* or self harm or adhd).tw.

48. (((substance or drug or alcohol) adj abuse) or "substance use" or "illegal drug use" or addict* or alcoholism or (problem* adj1 drinking)).tw.

49. or/ $1-48$

50. patient care planning/

51. ((care or action or treatment) adj plan*).tw.

52. decision making/

53. choice behavior/

54. (patient* adj7 (decision* or choice*)).tw.

55. patient preference/

Personalised care planning for adults with chronic or long-term health conditions (Review)

Copyright @ 2015 The Cochrane Collaboration. Published by John Wiley \& Sons, Ltd. 
56. (patient* adj3 (preference* or priorit* or value*)).tw.

57. (treatment adj (option* or choice*)).tw.

58. goals/

59. (goal* adj2 (set* or plan*)).tw.

60. patient centered care/

61. (patient adj (cent* ${ }^{*}$ red or focus*ed or oriented)).tw.

62. individualised medicine/

63. (individuali\#e? or individual\#ing or personali\#e? or personali\#ing or tailor or tailored or tailoring).tw.

64. or/50-63

65. cooperative behavior/

66. (patient* adj3 (participat* or involv*)).tw.

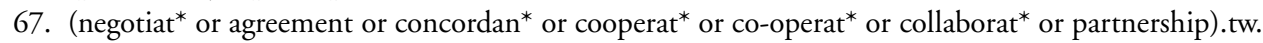

68. or/65-67

69. 64 and 68

70. patient participation/

71. (patient ${ }^{*}$ adj2 (empower* or activat*)).tw.

72. ((shared or joint or informed or collaborative) adj2 decision making).tw.

73. ((involv* or participat*) adj3 (choice* or decision*)).tw.

74. (decision adj (aid* or support or tool*)).tw.

75. patient provider agreement*.tw.

76. decisional self efficacy.tw.

77. (personal budget* or direct payment ${ }^{*}$ ).tw.

78. (record access or patient held record*).tw.

79. ((self management or self care) adj2 support*).tw.

80. or/69-79

81. 49 and 80

82. randomised controlled trial.pt.

83. controlled clinical trial.pt.

84. randomised.ab.

85. placebo.ab.

86. clinical trials as topic.sh.

87. randomly.ab.

88. trial.ti.

89. or $/ 82-88$

90. exp animals/ not humans.sh.

91. 89 not 90

92. 81 and 91

Embase: 
1

chronic.mp.Multimedia(41469)

1193893

2

((persistent or long* term or ongoing or degenerative) adj3 (disease* or ill* or condition* or insufficienc* or disorder*)).tw.Multimedia (964)

47182

3

long term care/Multimedia(791)

86414

4

long* term care.tw.Multimedia(42583)

16373

5

exp cardiovascular disease/Multimedia(1325)

2892644

6

(heart disease* or heart failure or myocardial ischemia or coronary disease* or coronary artery disease* or myocardial infarction or hypertension or high blood pressure).tw.Multimedia(11507)

822423

7

sickle cell.mp.Multimedia(745)

28624

8

exp chronic obstructive lung disease/Multimedia(3138)

68712

9

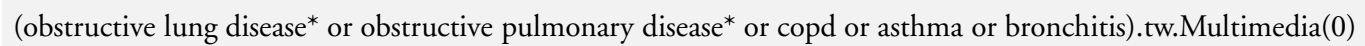

206623

10

lung emphysema/ or emphysema/Multimedia(4273)

26391

11

emphysema.tw.Multimedia(27099)

22105

12

(cystic fibrosis or respiratory distress).mp.Multimedia(14905)

117951

13

("degenerative disease/" and "exp cerebrovascular disease/").mp. [mp=title, abstract, subject headings, heading word, drug trade name, original title, device manufacturer, drug manufacturer, device trade name, keyword]Multimedia(1304)

0

14

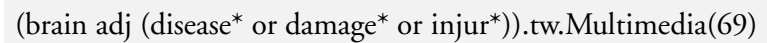

70039

15

(cerebrovascular or brain ischemia or cerebral infarction or carotid artery disease* or stroke or epilep* or seizure*).tw.Multimedia (13394)

417021

16

Personalised care planning for adults with chronic or long-term health conditions (Review)

Copyright $\odot 2015$ The Cochrane Collaboration. Published by John Wiley \& Sons, Ltd. 
(neurodegenerative or Huntington* or Parkinson* or amyotrophic lateral sclerosis or multiple sclerosis or motor neuron disease).tw. Multimedia(1200)

216767

17

(paralys* or quadriplegi* or tetraplegi* or paraplegi* or locked-in syndrome).tw.Multimedia(1768)

63002

18

((communication or learning or consciousness or perceptual or speech or voice or vision or hearing or psychomotor) adj disorder*). tw.Multimedia(17946)

8558

19

(hearing loss or hearing aid* or deaf* or blind* or stutter*).tw.Multimedia(1599)

335955

20

down* syndrome.tw.Multimedia(32938)

20827

21

cerebral palsy.tw.Multimedia(16626)

18883

22

exp gastrointestinal diseases/Multimedia(13895)

63741

23

(gastroenter* or intestinal or bowel or colonic).tw.Multimedia(2692)

432591

24

kidney failure/Multimedia(7815)

99730

25

((renal or kidney) adj (failure* or insufficienc*)).tw.Multimedia(12246)

121257

26

exp diabetes mellitus/Multimedia(181990)

567599

27

(diabetes or diabetic*).tw.Multimedia(5638)

535423

28

exp nutritional disorder/Multimedia(6911)

536110

29

(underweight or malnutrition or malnourished or overweight or obes*).tw.Multimedia(6437)

272692

30

exp arthritis/Multimedia(39318)

329119

31

(arthritis or osteoarthritis or rheumati* or fibromyalgia).tw.Multimedia(3378)

244558

Personalised care planning for adults with chronic or long-term health conditions (Review)

Copyright $\odot 2015$ The Cochrane Collaboration. Published by John Wiley \& Sons, Ltd. 
exp neoplasm/Multimedia(121)

3319359

38

(cancer* or oncolog* $^{*}$ or neoplasm* or carcinom* or tumo? ${ }^{*}$ or malignan* or leuk?emia).tw.Multimedia(585) 2861707

39

exp Human immunodeficiency virus infection/Multimedia(3406)

296469

40

(hiv infect* or hiv disease*).tw.Multimedia(93687)

94757

41

exp mental disease/Multimedia(1043)

\section{4}

42

((mental* or psychiatr* or psychological $\left.{ }^{*}\right)$ adj (ill* or disorder* or disease* or distress* or disab* or problem* ${ }^{*}$ or health* or patient* ${ }^{*}$ treatment)).tw.Multimedia(13857)

218664

43

((personality or mood or dysthymic or cognit* or anxiety or stress or eating or adjustment or reactive or somatoform or conversion or behavior or perception or psycho* or impulse control or development* or attention deficit or hyperactivity or conduct or motor skills or movement or tic or substance related) adj disorder*).tw.Multimedia(25768)

146882

44

(psychos\#s or psychotic* or paranoi* or schizo* or neuros\#s or neurotic* or delusion* or depression or depressive or bipolar or mania or manic or obsessi* or compulsi* or panic or phobic or phobia or anorexia or bulimia or neurastheni* or dissociative or autis* or Asperger* or Tourette or dyslex* or affective or borderline or narcissis* or suicid* or self injur* or self harm or adhd).tw.Multimedia (99)

45

(((substance or drug or alcohol) adj abuse) or "substance use" or "illegal drug use" or addict* or alcoholism or (problem* adj1 drinking) ).tw.Multimedia(315)

142653

Personalised care planning for adults with chronic or long-term health conditions (Review)

Copyright $\odot 2015$ The Cochrane Collaboration. Published by John Wiley \& Sons, Ltd. 
46

1 or 2 or 3 or 4 or 5 or 6 or 7 or 8 or 9 or 10 or 11 or 12 or 13 or 14 or 15 or 16 or 17 or 18 or 19 or 20 or 21 or 22 or 23 or 24 or 25 or 26 or 27 or 28 or 29 or 30 or 31 or 32 or 33 or 34 or 35 or 36 or 37 or 38 or 39 or 40 or 41 or 42 or 43 or 44 or $45 \mathrm{Multimedia}$ (108)

11136325

47

patient care planning/Multimedia(135)

26543

48

((care or action or treatment) adj plan*).tw.Multimedia(693)

61446

49

decision making/Multimedia(0)

133248

50

(patient* adj7 (decision* or choice*)).tw.Multimedia(0)

62144

51

patient preference/Multimedia(20)

3624

52

(patient* adj3 (preference* or priorit* or value*)).tw.Multimedia(0)

49062

53

(treatment adj (option* or choice*)).tw.Multimedia(89)

73666

54

(goal* adj2 (set* or plan*)).tw.Multimedia(0)

5819

55

(patient adj (cent* ${ }^{*}$ red or focus*ed or oriented)).tw.Multimedia(50298)

12376

56

personalized medicine/Multimedia(12880)

6173

57

(individuali\#e? or individuali\#ing or personali\#e? or personali\#ing or tailor or tailored or tailoring).tw.Multimedia(7255)

99601

58

47 or 48 or 49 or 50 or 51 or 52 or 53 or 54 or 55 or 56 or 57 Multimedia(3116)

488955

59

cooperation/Multimedia(13470)

28755

60

(patient* adj3 (participat* or involv*)).tw.Multimedia(219)

73668

61

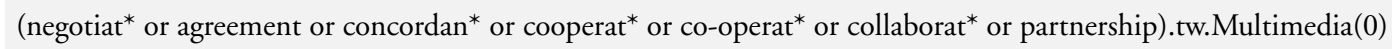

Personalised care planning for adults with chronic or long-term health conditions (Review)

Copyright $\odot 2015$ The Cochrane Collaboration. Published by John Wiley \& Sons, Ltd. 


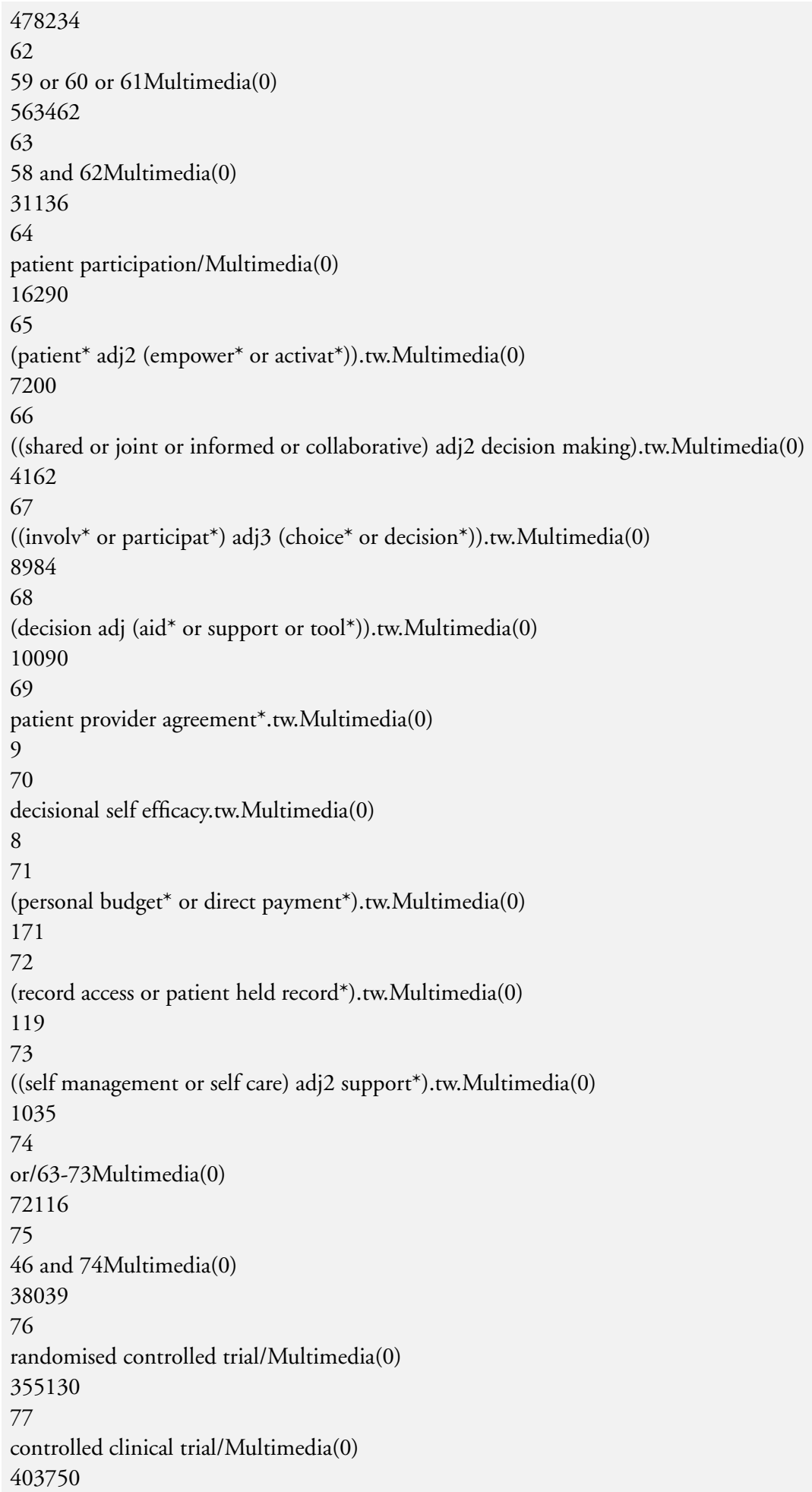


single blind procedure/ or double blind procedure/Multimedia(0)

136026

79

crossover procedure/Multimedia(0)

37988

80

random*.tw.Multimedia(0)

845821

81

placebo*.tw.Multimedia(0)

198596

82

$\left(\left(\right.\right.$ singl ${ }^{*}$ or doubl $\left.{ }^{*}\right)$ adj $\left(\right.$ blind $^{*}$ or mask $\left.\left.{ }^{*}\right)\right)$.tw.Multimedia(0)

161143

83

(crossover or cross over or factorial* or latin square).tw.Multimedia(0)

93133

84

(assign* or allocat* or volunteer*).tw.Multimedia(0)

481400

85

or/76-84Multimedia(0)

1484254

86

75 and 85 Multimedia( $(0)$

6811

\section{PsycINFO:}




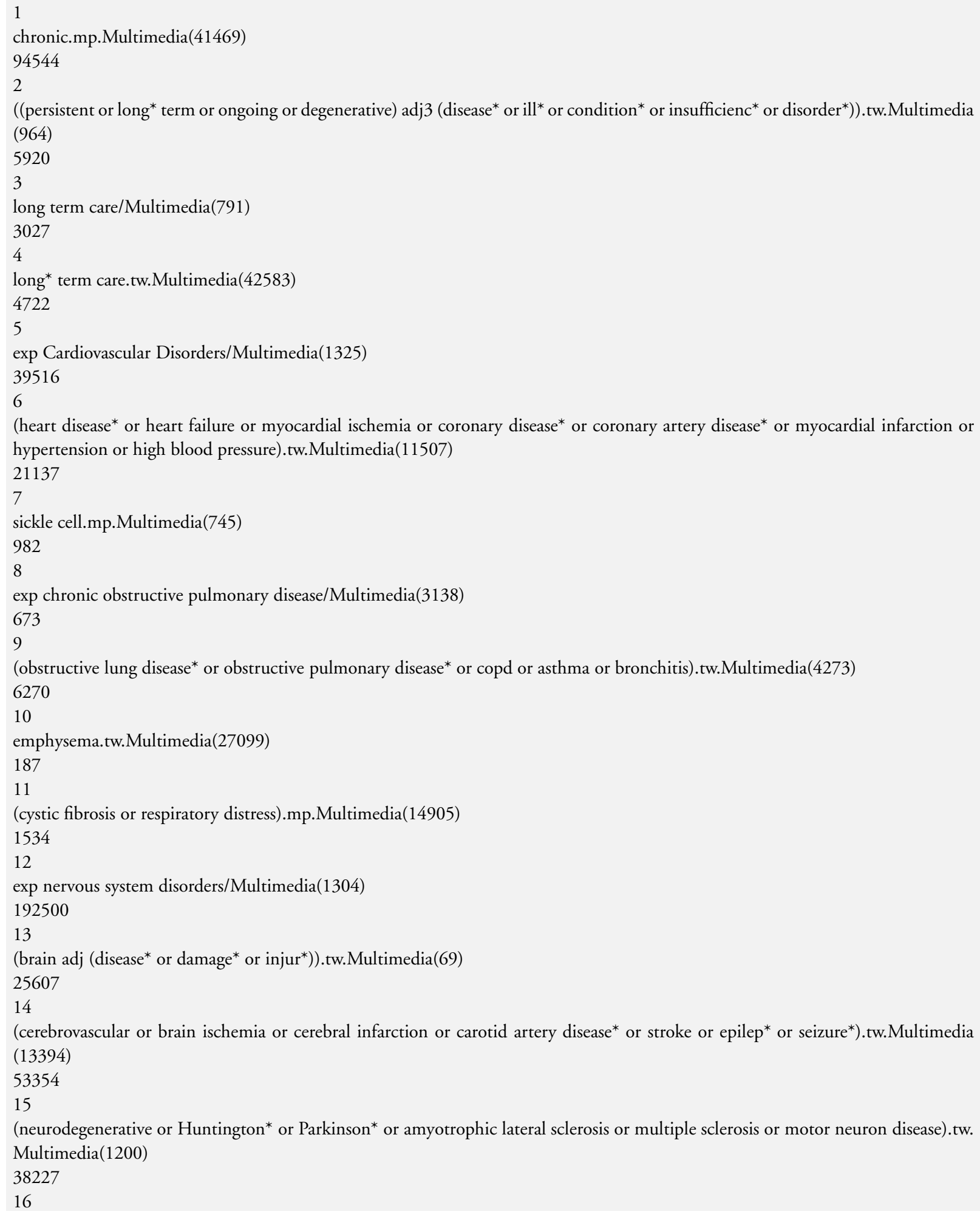
hypertension or high blood pressure).tw.Multimedia(11507)

Personalised care planning for adults with chronic or long-term health conditions (Review)

Copyright @ 2015 The Cochrane Collaboration. Published by John Wiley \& Sons, Ltd. 
(paralys* or quadriplegi* or tetraplegi* or paraplegi* or locked-in syndrome).tw.Multimedia(1768)

3518

17

((communication or learning or consciousness or perceptual or speech or voice or vision or hearing or psychomotor) adj disorder*). tw.Multimedia(17946)

5077

18

(hearing loss or hearing aid* ${ }^{*}$ or deaf* or blind* or stutter*).tw.Multimedia(1599)

54139

19

down* syndrome.tw.Multimedia(32938)

5746

20

cerebral palsy.tw.Multimedia(16626)

4063

21

exp Gastrointestinal Disorders/Multimedia(13895)

5340

22

(gastroenter* or intestinal or bowel or colonic).tw.Multimedia(2692)

4580

23

kidney diseases/Multimedia(7815)

1325

24

((renal or kidney) adj (failure* or insufficienc*)).tw.Multimedia(12246)

954

25

diabetes mellitus/Multimedia(181990)

3480

26

(diabetes or diabetic*).tw.Multimedia(5638)

17556

27

(underweight or malnutrition or malnourished or overweight or obes*).mp.Multimedia(6911) 26822

28

exp arthritis/Multimedia(6437)

2959

29

(arthritis or osteoarthritis or rheumati* or fibromyalgia).tw.Multimedia(39318)

6754

30

((back or neck) adj pain).tw.Multimedia(3378)

4155

31

exp Thyroid Disorders/Multimedia(537836)

1083

32

Personalised care planning for adults with chronic or long-term health conditions (Review)

Copyright $\odot 2015$ The Cochrane Collaboration. Published by John Wiley \& Sons, Ltd. 
thyroid.tw.Multimedia(1618)

3066

33

exp allergic disorders/Multimedia(1088)

722

34

(hypersensitivit* or allerg* or intolerance or anaphyla*).mp.Multimedia(2033)

6828

35

exp Neoplasms/Multimedia(1245)

31715

36

(cancer* ${ }^{*}$ on olog $^{*}$ or neoplasm* or carcinom* or tumo? ${ }^{*}$ or malignan* or leuk?emia).tw.Multimedia(121)

48707

37

exp HIV/Multimedia(585)

29689

38

(hiv infect* or hiv disease*).tw.Multimedia(3406)

11515

39

exp mental disorders/ or exp behavior disorders/Multimedia(100174)

508536

40

((mental* or psychiatr* or psychological $\left.{ }^{*}\right)$ adj (ill* or disorder* or disease* or distress* or disab* or problem* ${ }^{*}$ or health* or patient* or treatment)).tw.Multimedia(1043)

224025

41

((personality or mood or dysthymic or cognit* or anxiety or stress or eating or adjustment or reactive or somatoform or conversion or behavior or perception or psycho* or impulse control or development* or attention deficit or hyperactivity or conduct or motor skills or movement or tic or substance related) adj disorder*).tw.Multimedia(13857)

133493

42

(psychos\#s or psychotic* or paranoi* or schizo* or neuros\#s or neurotic* or delusion* or depression or depressive or bipolar or mania or manic or obsessi* or compulsi* or panic or phobic or phobia or anorexia or bulimia or neurastheni* or dissociative or autis* or Asperger* or Tourette or dyslex* or affective or borderline or narcissis* or suicid* or self injur* or self harm or adhd).tw.Multimedia (7953)

485242

43

(((substance or drug or alcohol) adj abuse) or "substance use" or "illegal drug use" or addict* or alcoholism or (problem* adj1 drinking) ).tw.Multimedia(205)

97963

44

1 or 2 or 3 or 4 or 5 or 6 or 7 or 8 or 9 or 10 or 11 or 12 or 13 or 14 or 15 or 16 or 17 or 18 or 19 or 20 or 21 or 22 or 23 or 24 or 25 or 26 or 27 or 28 or 29 or 30 or 31 or 32 or 33 or 34 or 35 or 36 or 37 or 38 or 39 or 40 or 41 or 42 or 43 Multimedia(315)

1125312

45

exp treatment planning/Multimedia(108)

3766

Personalised care planning for adults with chronic or long-term health conditions (Review)

Copyright () 2015 The Cochrane Collaboration. Published by John Wiley \& Sons, Ltd. 


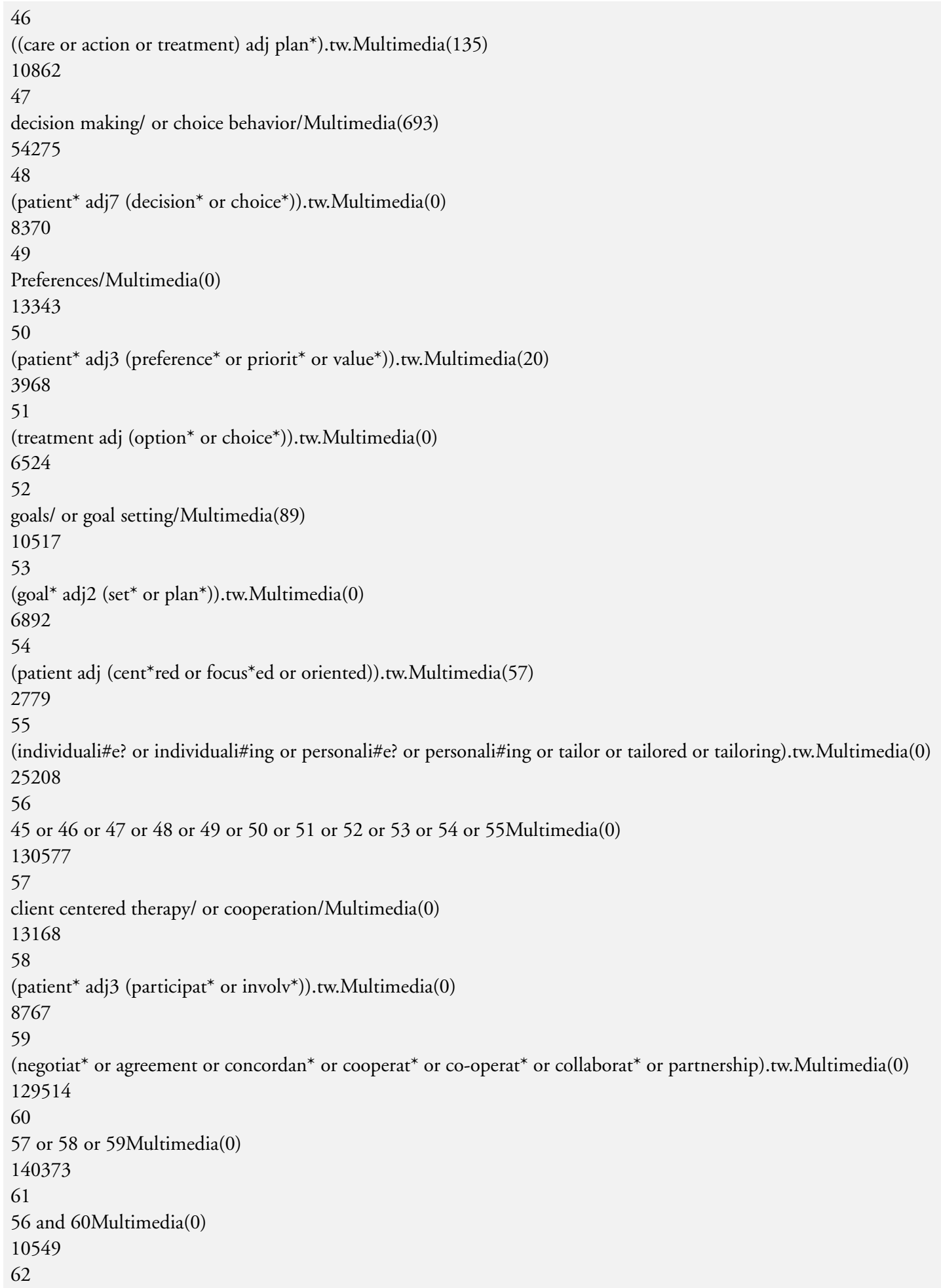




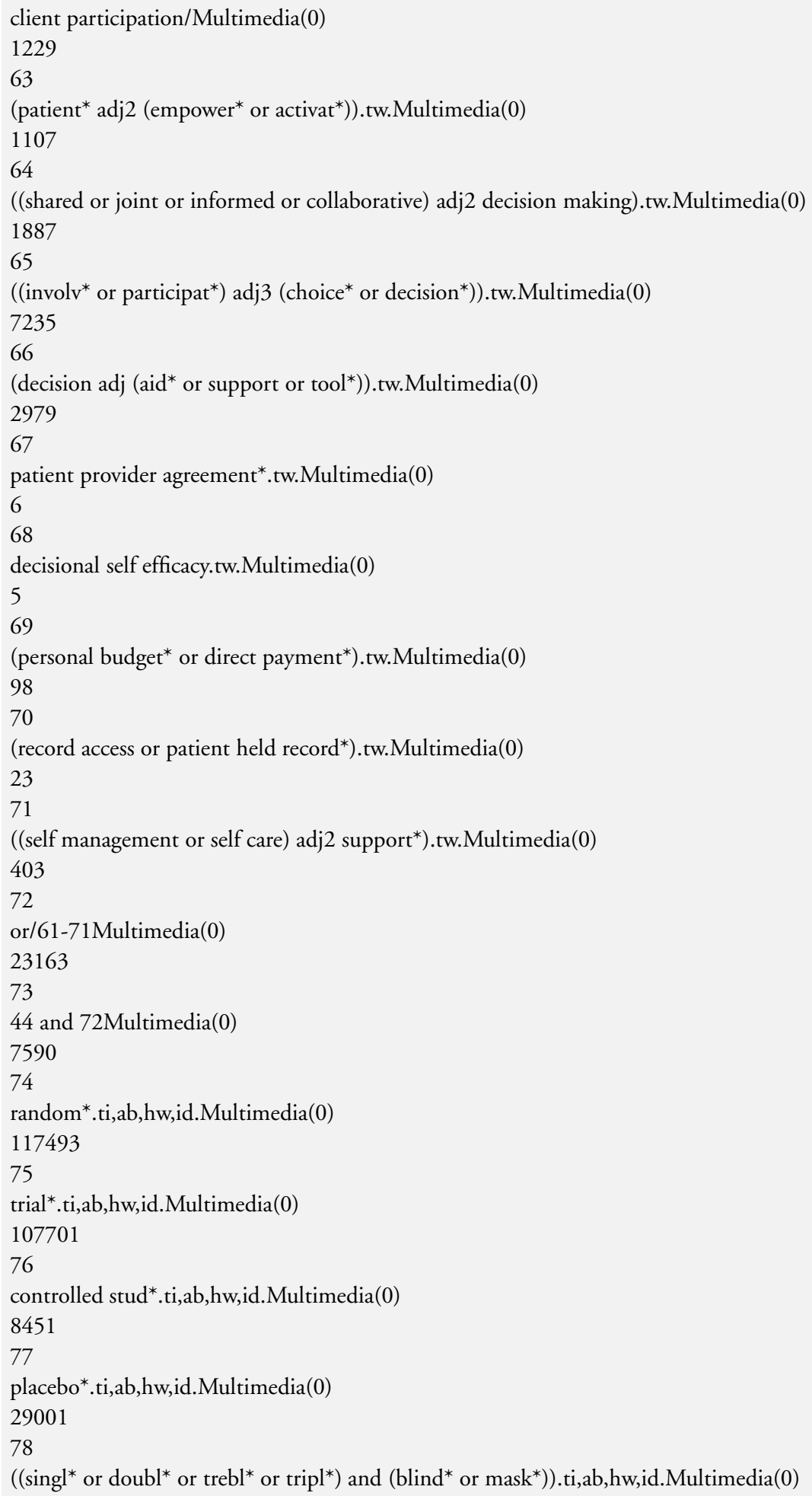




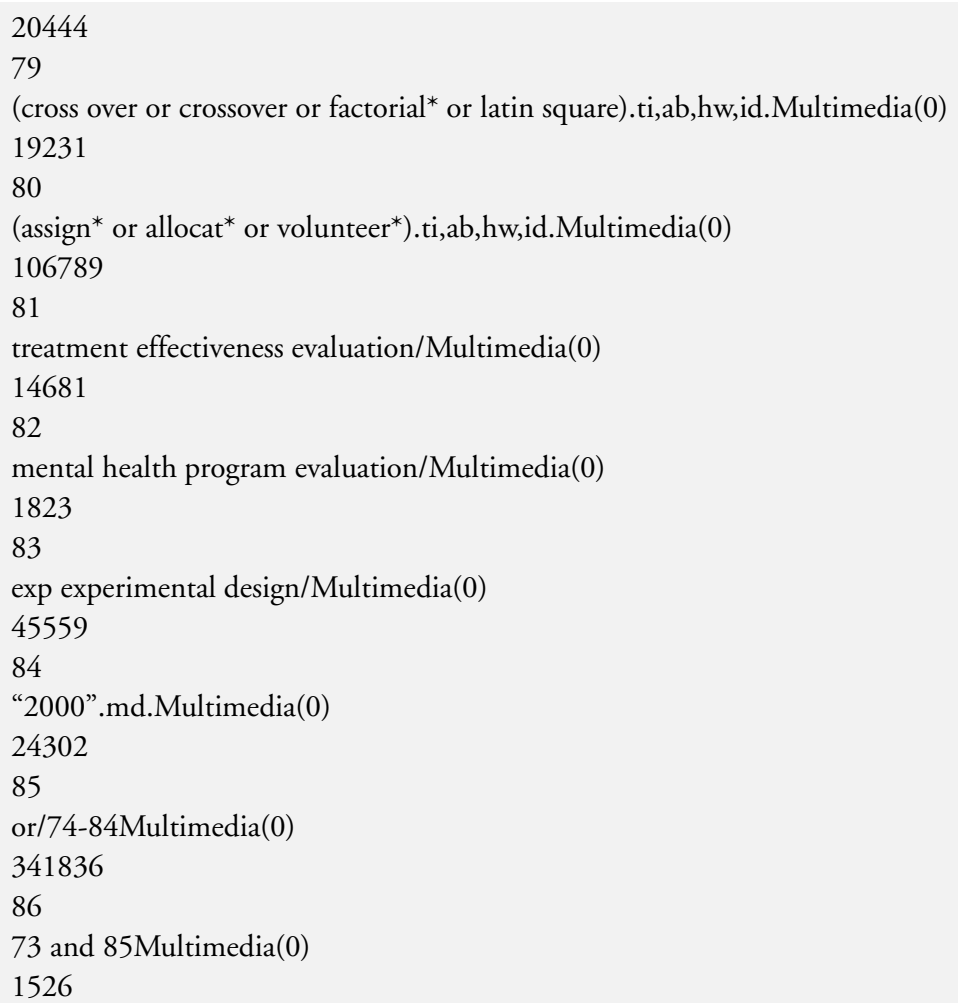

Trial registers:

Clinicaltrials.gov http://clinicaltrials.gov $-21^{\text {st }}$ June 2013

Main search: De-duplicated results $=508$

PCP search: results $=735$

The following terms were used in the Advanced search - Intervention search box - 8 separate searches.

"personal care plan" OR "personal care planning" OR "personal treatment plan" OR "personal treatment planning"

("decision making” OR choice OR choices OR care) AND (“patient involvement” OR "patient participation” OR "patient empowerment")

"shared decision making" OR "informed decision making" OR "joint decision making" OR "collaborative decision making" "patient provider agreement" OR "patient provider agreements" OR "decisional self efficacy” OR "personal budget” OR "personal budget” OR "direct payment" OR "direct payments" OR "record access" OR "patient held record” OR "patient held records" ("self management" OR "self care") AND support

("patient centered care" OR "patient centred care" OR "patient focused care" OR "patient oriented care”) AND (participation OR involvement)

("individualised care" OR "individualized care" OR “individualised medicine" OR "individualized medicine" OR "tailored care" OR "personalized care" OR "personalised care") AND (participation OR involvement)

"decision aid" OR decision aids" OR "decision support" OR "decision tool" OR "decision tools" (This search string gives loads of results - you might want to exclude from the search)

WHO ICTRP http://apps.who.int/trialsearch/ - 21 st June 2013

Main search: De-duplicated results $=106$

PCP search: Deduplicated results $=104$

Personalised care planning for adults with chronic or long-term health conditions (Review)

Copyright $\Subset 2015$ The Cochrane Collaboration. Published by John Wiley \& Sons, Ltd. 
"individualised care" OR “individualized care" OR “individualised medicine" OR “individualized medicine” OR "tailored care" OR "personalized care" OR "personalised care"

personal care plan OR personal care planning OR personal treatment plan OR personal treatment planning

shared decision making OR informed decision making OR "joint decision making" OR "collaborative decision making"

"patient provider agreement" OR "patient provider agreements" OR "decisional self efficacy" OR "personal budget" OR "personal budget" OR "direct payment" OR "direct payments" OR "record access" OR "patient held record" OR "patient held records"

"patient centered care" OR "patient centred care" OR "patient focused care" OR "patient oriented care"

self care support OR self management support

"decision aid” OR decision aids" OR “decision support” OR “decision tool” OR “decision tools"

\section{WHAT'S NEW}

Last assessed as up-to-date: 1 January 2014.

Date Event Description

23 March 2015 Amended minor correction to author affiliation

\section{CONTRIBUTIONS OFAUTHORS}

Angela Coulter conceived the review and drafted the protocol. Abi Eccles led the design of the search strategy and organised the consumer involvement. Sara Ryan, Abi Eccles and Angela Coulter selected studies for inclusion. Sara Ryan and Angela Coulter extracted the data. Rafael Perera led the statistical analysis. All authors contributed to the study design and analysis.

\section{DECLARATIONSOF INTEREST}

Angela Coulter: in addition to her part-time post at the University of Oxford, Angela acts as a paid consultant for the Informed Medical Decisions Foundation, a division of Healthwise, a global not-for-profit provider of health information.

Vikki Entwistle: none known

Abi Eccles: none known

Sara Ryan: none known

Sasha Shepperd: none known

Rafael Perera: none known 


\section{SOURCES OF SUPPORT}

\section{Internal sources}

- No sources of support supplied

\section{External sources}

- Department of Health Policy Research Programme, UK.

This is an independent report commissioned and funded by the Policy Research Programme in the Department of Health, England. The views expressed are not necessarily those of the Department.

\section{DIFFERENCES BETWEEN PROTOCOLANDREVIEW}

Some ambiguity in our inclusion/exclusion criteria was noted in respect of one trial (Kennedy 2013). This was designed as a trial of personalised care planning and self-management support, but in the event participating clinicians did not change their practice which remained the same in both the intervention group and the usual care group (Kennedy 2014). We therefore included this study in the review, but excluded it from the meta-analysis on the grounds that it was not a fair test of personalised care planning. Future iterations of this review should explicitly state that studies will be excluded if there is evidence that no change in practice (and hence no personalised care planning) actually occurred.

We changed the method used for dealing with missing data from that outlined in the protocol because we needed to impute standard deviations of change in some cases. Also, we originally planned to use a random-effects meta-analysis, but in the event this was not appropriate because of the small number of studies, so we used fixed-effect instead.

There were too few studies to group outcomes according to length of follow-up period as originally intended, so we took the final measurement in each case. We pooled outcomes if we felt the measures used were comparable, rather than by any other criterion. We added additional subgroup analyses to explore key characteristics (intensity, integration) once we had seen what the complex interventions actually involved. 